May 2004 • NREL/SR-510-33436

\title{
Evaluation of Biodiesel Fuel in an EMD GP38-2 Locomotive
}

S.G. Fritz

Southwest Research Institute

San Antonio, Texas
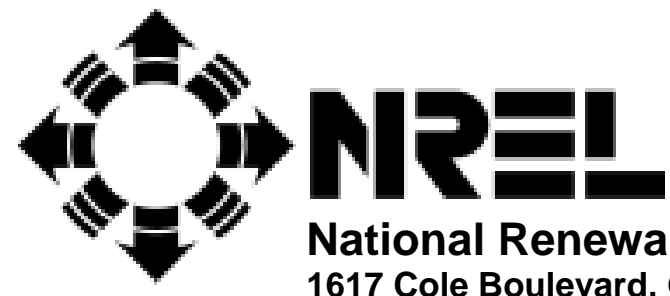

National Renewable Energy Laboratory 1617 Cole Boulevard, Golden, Colorado 80401-3393 303-275-3000 • www.nrel.gov

Operated for the U.S. Department of Energy

Office of Energy Efficiency and Renewable Energy

by Midwest Research Institute • Battelle

Contract No. DE-AC36-99-G010337 


\section{ERRATA SHEET}

NREL REPORT/PROJECT NUMBER: SR-510-33436

TITLE: Evaluation of Biodiesel Fuel in an EMD GP38-2 Locomotive

AUTHOR(S): S.G. Fritz, Southwest Research Institute

ORIGINAL PUBLICATION DATE: May 2004

DATE OF CORRECTIONS: August 2012

The following corrections were made to this report/document:

Appendices A-F were not included in the original publication.

Corrections to appendix references have also been made. No other changes are included in this revision. 


\title{
Evaluation of Biodiesel Fuel in an EMD GP38-2 Locomotive
}

\author{
S.G. Fritz \\ Southwest Research Institute \\ San Antonio, Texas
}

\section{NREL Technical Monitor: K.S. Tyson}

Prepared under Subcontract No. AXE-9-29057-01

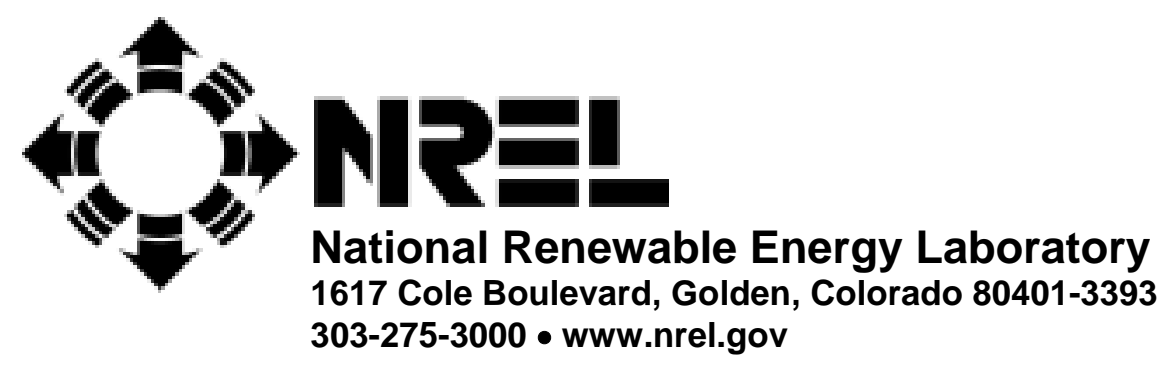

Operated for the U.S. Department of Energy

Office of Energy Efficiency and Renewable Energy

by Midwest Research Institute $\bullet$ Battelle

Contract No. DE-AC36-99-G010337 


\section{NOTICE}

This report was prepared as an account of work sponsored by an agency of the United States government. Neither the United States government nor any agency thereof, nor any of their employees, makes any warranty, express or implied, or assumes any legal liability or responsibility for the accuracy, completeness, or usefulness of any information, apparatus, product, or process disclosed, or represents that its use would not infringe privately owned rights. Reference herein to any specific commercial product, process, or service by trade name, trademark, manufacturer, or otherwise does not necessarily constitute or imply its endorsement, recommendation, or favoring by the United States government or any agency thereof. The views and opinions of authors expressed herein do not necessarily state or reflect those of the United States government or any agency thereof.

Available electronically at http://www.osti.gov/bridge

Available for a processing fee to U.S. Department of Energy and its contractors, in paper, from:

U.S. Department of Energy

Office of Scientific and Technical Information

P.O. Box 62

Oak Ridge, TN 37831-0062

phone: 865.576.8401

fax: 865.576 .5728

email: mailto:reports@adonis.osti.gov

Available for sale to the public, in paper, from:

U.S. Department of Commerce

National Technical Information Service

5285 Port Royal Road

Springfield, VA 22161

phone: 800.553 .6847

fax: 703.605.6900

email: orders@ntis.fedworld.gov

online ordering: http://www.ntis.gov/ordering.htm

Printed on paper containing at least $50 \%$ wastepaper, including $20 \%$ postconsumer waste 


\section{ACKNOWLEDGMENTS}

The work reported in this document was performed for the National Renewable Energy Laboratory, as outlined in SwRI Proposal No. 08-25148a, titled "Biodiesel Emissions from a Locomotive Engine," dated May 27, 1999. This project was performed by the Department of Emissions Research of the Automotive Products and Emissions Research Division under the supervision of Mr. Charles T. Hare. The Project Manager for SwRI was Mr. Steven G. Fritz, Principal Engineer in the Department of Emissions Research. SwRI technical personnel making significant contributions to the project were Mr. C. E. Grinstead, Research Technologist, Mr. S. Tenay, Senior Technician, Mr. C. Medelez, Lab Assistant, and Ms. Kathy Jack, Research Assistant.

The NREL technical contact was Dr. K. Shaine Tyson, Renewable Diesel Project Manager. The assistance of Mr. Ted Stewart of CSX Transportation for coordinating use of the EMD GP38-2 locomotive was greatly appreciated. 


\section{TABLE OF CONTENTS}

$\underline{\text { Page }}$

ACKNOWLEDGMENTS $\ldots \ldots \ldots \ldots \ldots \ldots \ldots \ldots \ldots \ldots \ldots \ldots \ldots \ldots \ldots$

LIST OF FIGURES $\ldots \ldots \ldots \ldots \ldots \ldots \ldots \ldots \ldots \ldots \ldots \ldots \ldots \ldots \ldots \ldots \ldots \ldots \ldots$

LIST OF TABLES $\ldots \ldots \ldots \ldots \ldots \ldots \ldots \ldots \ldots \ldots \ldots \ldots \ldots \ldots \ldots \ldots \ldots \ldots \ldots \ldots \ldots$

LIST OF ABBREVIATIONS $\ldots \ldots \ldots \ldots \ldots \ldots \ldots \ldots \ldots \ldots \ldots \ldots \ldots \ldots \ldots$ iv

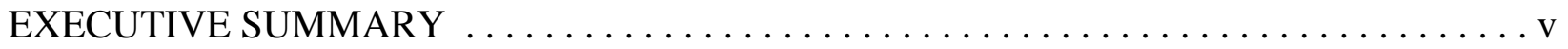

I. TECHNICAL APPROACH $\ldots \ldots \ldots \ldots \ldots \ldots \ldots \ldots \ldots \ldots \ldots \ldots \ldots \ldots$

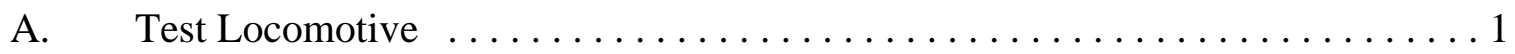

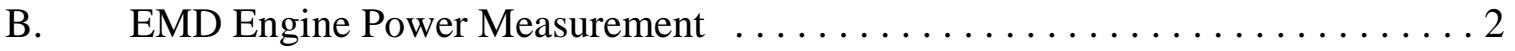

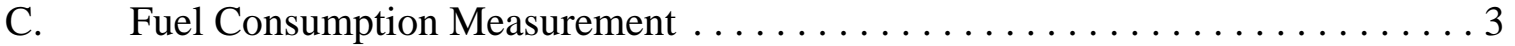

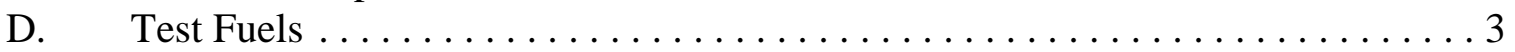

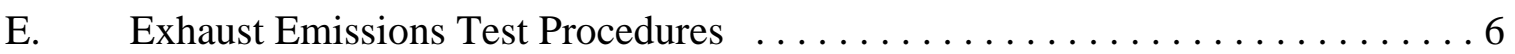

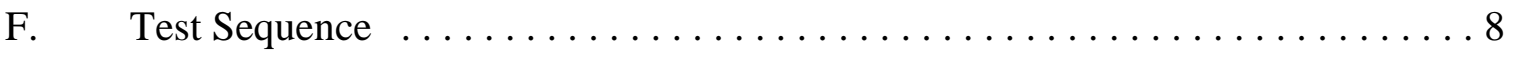

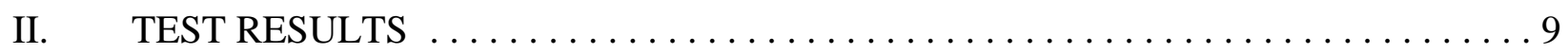

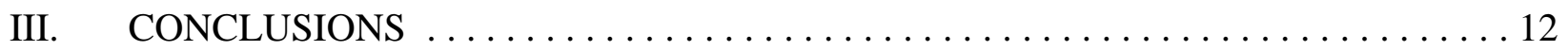




\section{LIST OF FIGURES}

Figure

Page

1 CSX No. 2629 at the SwRI Locomotive Test Center ...................... 1

$2550-$ Gallon Fuel Storage Tanks Located Next to Locomotive $\ldots \ldots \ldots \ldots \ldots$

\section{LIST OF TABLES}

Table

Page

ES-1 Summary of Locomotive Tests Using Biodiesel Fuel Blends $\ldots \ldots \ldots \ldots \ldots \ldots \ldots$ v

1 EMD 16-645-E Engine Specifications $\ldots \ldots \ldots \ldots \ldots \ldots \ldots \ldots \ldots \ldots \ldots \ldots \ldots \ldots \ldots \ldots \ldots \ldots$

2 Properties of Diesel Fuels Used in Locomotive Testing ..................... 4

3 Duty Cycles Used to Compute Weighted Composite Emissions $\ldots \ldots \ldots \ldots \ldots \ldots$

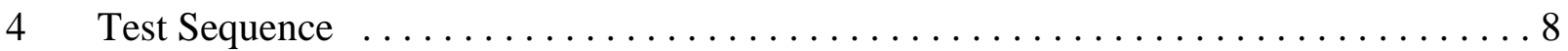

5 CSX No. 2629 Test Summary ................................. 10 


\section{LIST OF ABBREVIATIONS}

$\begin{array}{ll}\text { AAR } & \text { Association of American Railroads } \\ \text { API } & \text { American Petroleum Institute } \\ \text { ASTM } & \text { American Society for Testing and Materials } \\ \text { BSFC } & \text { brake specific fuel consumption } \\ \text { BTU } & \text { British Thermal Units } \\ \text { CFR } & \text { Code of Federal Regulations } \\ \text { cm } & \text { centimeter } \\ \text { CO } & \text { carbon monoxide } \\ \mathrm{CO}_{2} & \text { carbon dioxide } \\ \text { cSt } & \text { centistokes } \\ \text { EMD } & \text { Electro-Motive Division of General Motors Corporation } \\ \text { EP } & \text { end point } \\ \text { EPA } & \text { Environmental Protection Agency } \\ \text { oF } & \text { degrees Fahrenheit } \\ \text { f/a } & \text { mass fuel to dry air ratio } \\ \text { FBP } & \text { final boiling point } \\ \text { FTP } & \text { Federal Test Procedure } \\ \text { g } & \text { gram } \\ \text { gal } & \text { gallon } \\ \mathrm{H}_{2} \mathrm{O} & \text { water } \\ \mathrm{HC} & \text { hydrocarbons } \\ \mathrm{HFID} & \text { heated flame ionization detector } \\ \text { hp } & \text { horsepower } \\ \text { hr } & \text { hour } \\ \text { IBP } & \text { initial boiling point } \\ \text { in } & \text { inch } \\ \text { kW } & \text { kilowatt } \\ \text { lb } & \text { pound } \\ \text { min } & \text { minute } \\ \text { mm } & \text { millimeter } \\ \mathrm{NREL} & \text { National Renewable Energy Laboratory } \\ \mathrm{NDIR} & \text { non-dispersive infrared } \\ \mathrm{NO} & \text { oxides of nitrogen } \\ \mathrm{O}_{2} & \text { Oxygen } \\ \text { PHS } & \text { Public Health Service } \\ \mathrm{PM} & \text { particulate matter } \\ \text { rpm } & \text { revolutions per minute } \\ \text { sec } & \text { seconds } \\ \mathrm{SwRI} & \text { Southwest Research Institute } \\ \text { TDC } & \text { top dead center } \\ \text { wt } & \text { weight } \\ \% & \text { percent } \\ & \end{array}$




\section{EXECUTIVE SUMMARY}

This report documents results of fuel consumption and exhaust emission tests performed on a 2,000 hp EMD GP38-2 locomotive operating on four different fuels. No operational problems were observed by SwRI on any of the test fuels, and the locomotive engine was able to produce within $1 \%$ to $2 \%$ of full rated power while operating on the biodiesel blends. Locomotive tests were performed at the SwRI Locomotive Exhaust Emissions Test Center in October, 2000.

Table ES-1 summarizes the average EPA Line-Haul and Switch duty-cycle weighted exhaust emissions from triplicate tests using a diesel fuel meeting EPA locomotive certification test specifications, on a CARB diesel fuel, with a blend of $20 \%$ bio-fuel into the EPA locomotive certification fuel (B20), and a second blend of 20 percent bio-fuel with the CARB diesel (C20). The $4 \%$ to $6 \%$ increase in $\mathrm{NO}_{\mathrm{X}}$ emissions with $\mathrm{B} 20$ fuel compared to EPA locomotive certification diesel is consistent with B20 tests performed in other engine test programs. The lack of PM response with any of the test fuels is attributed to the fact that most of the PM emissions from this two-stroke EMD engine are lubricating-oil derived. Long term durability and reliability were not addressed in this study.

Table ES-1. Summary of Locomotive Tests Using Biodiesel Fuel Blends

\begin{tabular}{|c|c|c|c|c|}
\hline Fuel & $\mathrm{HC}$ & CO & $\mathrm{NO}_{\mathrm{x}}$ & PM \\
\hline \multicolumn{5}{|c|}{ EPA Line-Haul Duty-Cycle Weighted Emissions, g/hp-hr ${ }^{\text {a }}$} \\
\hline EPA Loco. Cert. Diesel & 0.64 & 5.4 & 12.4 & 0.46 \\
\hline CARB diesel & 0.64 & 4.3 & 12.3 & 0.46 \\
\hline B20 & 0.64 & 4.5 & 13.1 & 0.50 \\
\hline $\mathrm{C} 20$ & 0.64 & 4.0 & 12.8 & 0.48 \\
\hline \multicolumn{5}{|c|}{ EPA Switch Duty-Cycle Weighted Emissions, g/hp-hr ${ }^{a}$} \\
\hline EPA Loco. Cert. Diesel & 0.82 & 2.2 & 12.8 & 0.38 \\
\hline CARB diesel & 0.76 & 1.8 & 12.5 & 0.34 \\
\hline B20 & 0.78 & 2.0 & 13.5 & 0.37 \\
\hline $\mathrm{C} 20$ & 0.73 & 1.8 & 13.1 & 0.37 \\
\hline
\end{tabular}




\section{TECHNICAL APPROACH}

Locomotive exhaust emission tests for this project were performed by Southwest Research Institute (SwRI) at the Locomotive Exhaust Emissions Test Center in San Antonio, Texas. This facility was established in August 1993 in cooperation with the Association of American Railroads (AAR).

Presented below is an overview of the technical approach used to conduct locomotive exhaust emissions testing. Included is a description of the locomotive selected for testing, engine power measurements, fuel consumption measurements, the test fuels, exhaust emissions test procedures, analytical procedures, particulate measurement procedures, and smoke opacity test procedures.

\section{A. Test Locomotive}

The locomotive tested in this project was CSX No. 2629, a model GP38-2, manufactured in 1973 by the Electro-Motive Division (EMD) of General Motors Corporation. CSX No. 2629 shown in Figure 1, is generally classified as a "road-switcher" locomotive, meaning it serves as a local switcher locomotive as well as a line-haul locomotive.

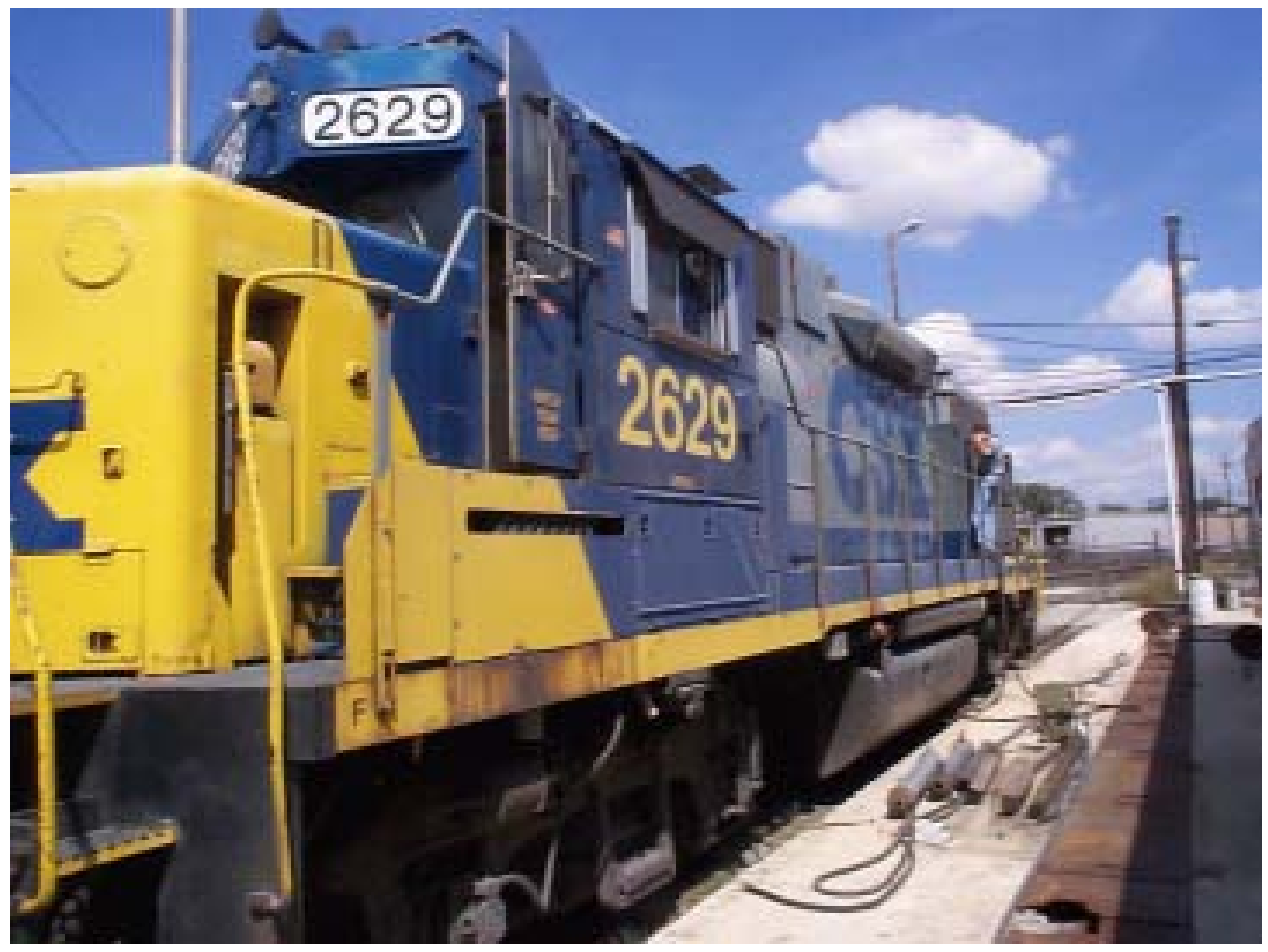

FIGURE 1. CSX NO. 2629 at the SWRI Locomotive Test Center

The GP-38 locomotive was equipped with a 2,000 hp roots-blown EMD model 16-645-E diesel engine (SN 84-B3-1505). Table 1 gives the specifications for the EMD engine. Immediately 
before shipping the locomotive to SwRI, the locomotive was serviced at CSX's Cumberland, Maryland shop. Service included replacing the fuel injectors, changing the fuel, lubricating oil and engine intake air filters, and changing the engine lubricating oil.

Table 1. EMD 16-645-E Engine Specifications

\begin{tabular}{|c|c|}
\hline Type & 2-stroke, uniflow scavenged diesel \\
\hline Aspiration & roots-blown, non-turbocharged \\
\hline Bore, in. & $9_{1 / 16}$ \\
\hline Stroke, in. & (254) \\
\hline No. of Cylinders & 16 \\
\hline Displacement, cu. in./cyl. $\quad$ (L) & $(10.6)$ \\
\hline Total displacement cu. in. $\quad(\mathrm{L})$ & 10,320 \\
\hline Compression Ratio & $16: 1$ \\
\hline Fuel Injection System & Unit Injector \\
\hline Rated Power, bhp & $(1,491)$ \\
\hline Rated Speed, rpm & 900 \\
\hline
\end{tabular}

\section{B. EMD Engine Power Measurement}

Most line-haul locomotives are equipped with the "dynamic brake" feature in which the electric motors used for traction are reverse-excited to become generators for slowing the train. The electrical power generated is dissipated in resistance grids. Locomotives with the self-load feature can dissipate the main alternator power into these "dynamic brake" resistance grids. The CSX locomotive tested in this program was equipped with dynamic brake grids capable of dissipating the full engine power, and these grids were used to load the stationary locomotive.

Engine flywheel (gross) power was determined by direct measurement of the main alternator voltage and current, plus auxiliary power values obtained from load box testing procedures published by EMD. EPA locomotive test procedures call for direct measurement of main generator voltage and current, and for the manufacturer to apply a known generator efficiency to calculate gross power. In this case, a single alternator efficiency of $93.9 \%$ was used for all test points, following EMD load box testing procedures. Power for the various auxiliary systems (auxiliary generator, traction motor blower, inertial separator blower, and radiator fans) was computed using published EMD test procedures. The air compressor on the locomotive was disabled during testing, and compressed air was supplied to the locomotive.

Power and fuel rates were recorded as observed values. Corrected brake-specific fuel 
consumption (BSFC) values were computed using published EMD power correction factors. Observed power was used to report brake-specific exhaust emissions in $\mathrm{g} / \mathrm{hp}$-hr.

\section{Fuel Consumption Measurement}

Fuel consumption was measured on a mass basis, using a mass flow meter. The system was equipped with a heat exchanger to control fuel supply temperature to $90 \pm 10^{\circ} \mathrm{F}$. Hot return fuel, which would normally return to the locomotive on-board fuel tank, was cooled before returning to the fuel measurement reservoir ("day tank") to assure a consistent fuel supply temperature at the engine.

\section{Test Fuels}

Four fuels were included in this study, and selected properties of the fuels are summarized in Table 2. Baseline tests were performed using diesel fuel meeting EPA specifications for locomotive emissions test fuel given in the Code of Federal Regulations, Title 40, Part 92, Section 92.113. This "locomotive cert fuel" had a sulfur content of 0.30 percent, which is the midpoint of the EPA specification of $0.2 \%$ to $0.4 \%$ sulfur for locomotive testing. Table 2 also gives the specifications for EPA locomotive test fuel.

The second fuel used in this program was a "CARB fuel." This fuel was used in a recently completed locomotive fuel-effects program performed by SwRI for CARB. ${ }^{1}$ The "CARB" fuel was a 50/50 blend of two commercially available CARB-approved fuels, one batch was from the Texaco refinery in Los Angeles, California, and the second batch was from the ARCO refinery in South Gate, California. The properties of the CARB fuel, as reported in Table 2, were considered by ARB to be representative of commercially available on-highway fuel sold in California.

The third and fourth fuels tested in this program were $20 \%$ blends (by volume) bio-fuel blended into the two different base fuels. These are referred to as "B20," which was $20 \%$ bio-fuel blended with the "locomotive cert fuel," and "C20," which was $20 \%$ biodiesel blended with the CARB diesel fuel.

The bio-fuel used in this program was supplied by Griffin Industries, Inc. of Cold Spring, Kentucky. Eleven 55-gallon drums of Bio "G-3000TM" were supplied to SwRI for blending. Blended fuels were stored in 550-gallon totes that were located next to the locomotive, as shown in Figure 2.

\footnotetext{
1 Fritz, S.G., "Diesel Fuel Effects on Locomotive Exhaust Emissions," SwRI Report No. 08-02062 (October 2000).
} 
Table 2. Properties of Diesel Fuels Used in Locomotive Testing

\begin{tabular}{|c|c|c|c|c|c|c|}
\hline Determinations & $\begin{array}{c}\text { ASTM } \\
\text { Test Method }\end{array}$ & $\begin{array}{l}\text { Locomotive } \\
\text { Cert. Fuel } \\
\text { EM-2834-F }\end{array}$ & $\begin{array}{c}\text { B20 } \\
\text { EM-3004-F }\end{array}$ & $\begin{array}{l}\text { CARB Fuel } \\
\text { EM-2663-F }\end{array}$ & $\begin{array}{c}\text { C20 } \\
\text { EM-3005-F }\end{array}$ & $\begin{array}{c}\text { EPA } \\
\text { Locomotive }^{\text {Spec. }}{ }^{\text {a }}\end{array}$ \\
\hline $\begin{array}{l}\text { API Gravity @ 60 } \\
\text { specific gravity } \\
\text { density (lb/gal) }\end{array}$ & D4052 & $\begin{array}{c}34.4 \\
0.8529 \\
7.12\end{array}$ & $\begin{array}{c}33.2 \\
0.8594 \\
7.17\end{array}$ & $\begin{array}{c}39.1 \\
0.8295 \\
6.92\end{array}$ & $\begin{array}{c}36.8 \\
0.8409 \\
7.02\end{array}$ & $\begin{array}{c}32-37 \\
\text { ns } \\
\text { ns }\end{array}$ \\
\hline Viscosity @ 40ㄷ (cSt) & D445-83 & 2.77 & 3.17 & 2.46 & 2.82 & $2.0-3.2$ \\
\hline Sulfur (Wt\%) & D2622-94 & 0.2996 & 0.2264 & 0.005 & 0.004 & $0.2-0.4$ \\
\hline Cetane Index & D976 & 47.7 & 49.0 & 52.0 & 53.4 & $40-48$ \\
\hline Cetane Index & D4737 & 47.6 & 47.6 & 53.1 & 53.4 & ns \\
\hline Cetane Number & D613-84 & 45.7 & 47.0 & 51.0 & 50.5 & $40-48$ \\
\hline $\begin{array}{l}\text { Heat of Combustion } \\
\text { Gross (BTU/lb) } \\
\text { Net (BTU/lb) } \\
\text { Gross (BTU/gal) } \\
\text { Net (btu/gal) }\end{array}$ & D240 & $\begin{array}{c}19,470 \\
18,272 \\
138,600 \\
130,100\end{array}$ & $\begin{array}{c}18,942 \\
17,772 \\
135,900 \\
127,500\end{array}$ & $\begin{array}{c}19,715 \\
18,479 \\
136,400 \\
127,900\end{array}$ & $\begin{array}{c}19,149 \\
17,940 \\
134,400 \\
125,900\end{array}$ & $\begin{array}{l}\text { ns } \\
\text { ns } \\
\text { ns } \\
\text { ns }\end{array}$ \\
\hline $\begin{array}{l}\text { Carbon-Hydrogen Ratio } \\
\% \text { Carbon } \\
\% \text { Hydrogen } \\
\text { Hydrogen/Carbon Ratio }\end{array}$ & D3178 & $\begin{array}{c}86.77 \\
13.23 \\
1.82\end{array}$ & $\begin{array}{c}84.13 \\
12.82 \\
1.82\end{array}$ & $\begin{array}{c}86.37 \\
13.63 \\
1.88\end{array}$ & $\begin{array}{c}83.76 \\
13.25 \\
1.89\end{array}$ & $\begin{array}{l}\text { ns } \\
\text { ns } \\
\text { ns }\end{array}$ \\
\hline $\begin{array}{l}\text { SFC Aromatics } \\
\text { Total Mass \% } \\
\text { Total Volume Percent }{ }^{\mathrm{b}} \\
\text { PNA Mass } \%\end{array}$ & D5186-96 & $\begin{array}{c}30.6 \\
29.4 \\
\text { nd }\end{array}$ & nd & $\begin{array}{l}22.4 \\
21.8 \\
1.7 \\
\end{array}$ & nd & $27 \mathrm{~min}$. \\
\hline $\begin{array}{l}\text { Hydrocarbon Type } \\
\text { Aromatics (\%) } \\
\text { Olefins (\%) } \\
\text { Saturates (\%) } \\
\end{array}$ & D1319-84 & $\begin{array}{c}36.2 \\
1.2 \\
62.6 \\
\end{array}$ & nd & $\begin{array}{c}22.4 \\
2.0 \\
75.6 \\
\end{array}$ & nd & $\begin{array}{l}\text { ns } \\
\text { ns } \\
\text { ns }\end{array}$ \\
\hline Flash Point $\left({ }^{\circ} \mathrm{F}\right)$ & D93-80 & 166 & 172 & 167 & 170 & $130 \mathrm{~min}$. \\
\hline Distillation & $\begin{array}{c}\text { D86-96 } \\
\text { \% Recovered } \\
\text { IBP } \\
10 \\
50 \\
90 \\
\text { EP }\end{array}$ & $\begin{array}{l}382 \\
423 \\
519 \\
627 \\
676\end{array}$ & $\begin{array}{l}374 \\
431 \\
553 \\
639 \\
670\end{array}$ & $\begin{array}{l}368 \\
413 \\
490 \\
606 \\
659\end{array}$ & $\begin{array}{l}376 \\
418 \\
533 \\
633 \\
663\end{array}$ & $\begin{array}{l}\text { Temp. }{ }^{\circ} \mathrm{F} \\
340-400 \\
400-460 \\
470-540 \\
560-630 \\
610-690\end{array}$ \\
\hline \multicolumn{7}{|l|}{ Note: } \\
\hline
\end{tabular}




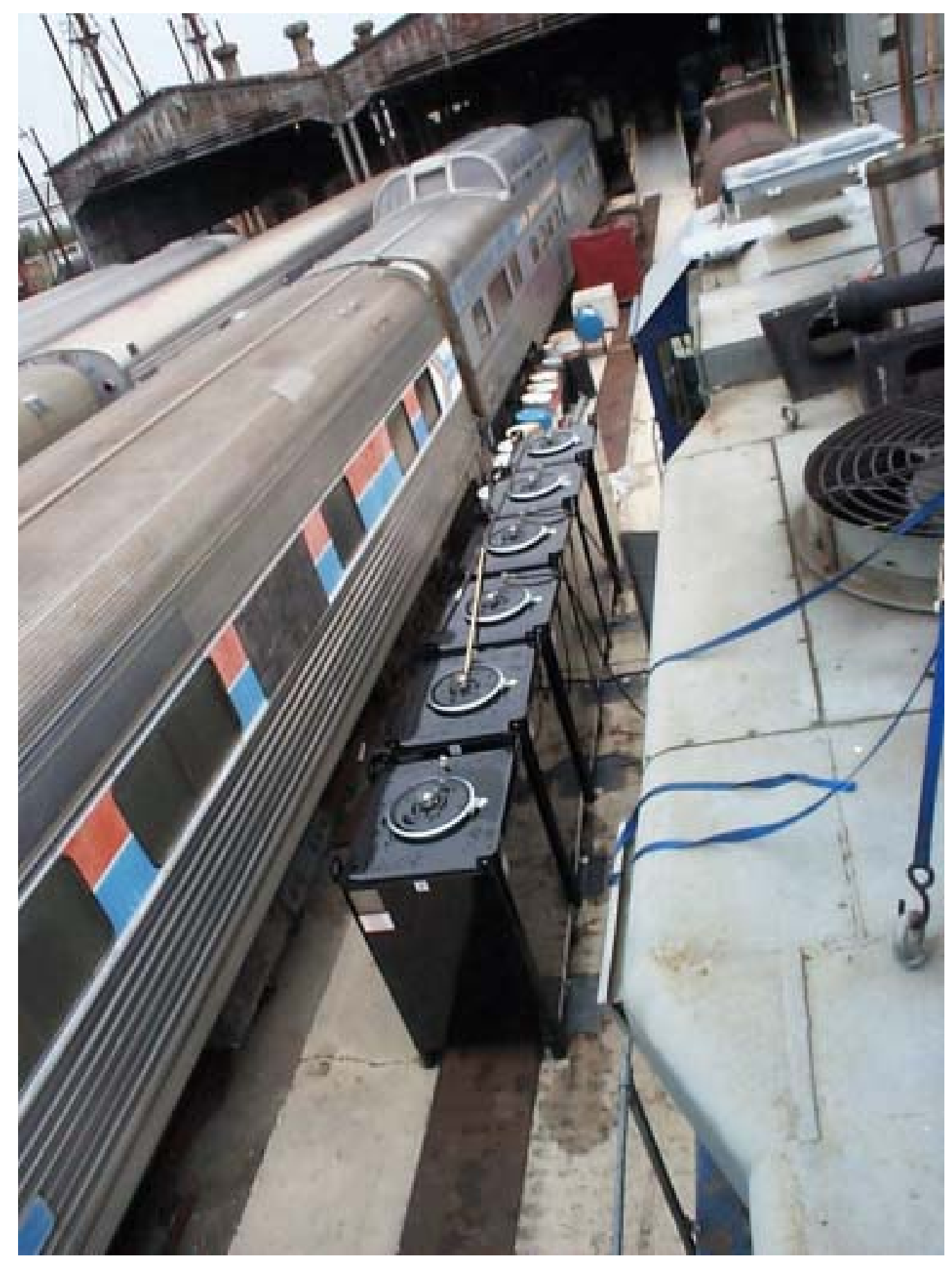

Figure 2. 550-Gallon Fuel Storage Tanks Located Next to Locomotive 


\section{E. Exhaust Emissions Test Procedure}

Exhaust emission tests were performed using the locomotive Federal Test Procedure (FTP) detailed in the U.S. Code of Federal Regulations (CFR), Title 40, Part 92, "Emission Standards for Locomotives and Locomotive Engines."

\section{Gaseous Emissions Sampling}

A gaseous sample probe was designed using EPA locomotive test specifications. Gaseous emissions were sampled within the exhaust stack extension installed on the roof of the locomotive. A heated line was used to transfer the raw exhaust sample to the emission instruments for analysis. Measured gaseous emissions included hydrocarbons (HC), carbon monoxide $(\mathrm{CO})$, carbon dioxide $\left(\mathrm{CO}_{2}\right)$, oxygen $\left(\mathrm{O}_{2}\right)$, and oxides of nitrogen $\left(\mathrm{NO}_{\mathrm{X}}\right)$.

The hydrocarbon concentration in the raw exhaust was determined using a Rosemount Analytical model 402 heated flame ionization detector (HFID), calibrated on propane. $\mathrm{NO}_{\mathrm{X}}$ concentration in the raw exhaust was measured with a Rosemount model 955 chemiluminescent analyzer. $\mathrm{NO}_{\mathrm{X}}$ correction factors for engine intake manifold air temperature and ambient air humidity were applied as specified by EPA in 40 CFR $\$ 86.132(\mathrm{~d})$. Concentrations of CO and $\mathrm{CO}_{2}$ in the raw exhaust were determined by non-dispersive infrared (NDIR) instruments.

Gaseous mass emission rates were computed using the measured concentration, the observed (measured) fuel consumption rate, and calculated engine air flow. Following the FTP, engine air flow was not directly measured in this test program. Instead, engine air flow was determined using a carbon balance of the carbon-containing constituents in the exhaust $\left(\mathrm{CO}_{2}, \mathrm{CO}\right.$, and $\left.\mathrm{HC}\right)$ to compute the fuel/air ratio (f/a). Engine air flow rate was then computed using the measured fuel consumption rate and the computed f/a ratio.

\section{Particulate Emissions Sampling}

Particulate emissions were measured at each test point using a "split then dilute" technique, in which a portion of the raw locomotive exhaust was "split" from the total exhaust and mixed with filtered dilution air in a 10-inch diameter dilution tunnel. The split sample of raw exhaust was transferred to the dilution tunnel through a 2-inch diameter stainless steel tube that was insulated and electrically heated to $375^{\circ} \mathrm{F}$. Using filtered dilution air, the raw exhaust was diluted and the temperature reduced to less than $125^{\circ} \mathrm{F}$ before sampling for particulate emissions.

A particulate sample was extracted from the dilution tunnel through sample probes fitted with stainless steel filter holders. Particulate was accumulated on $90 \mathrm{~mm}$ fluorocarbon-coated glass fiber filters (Pallflex T60A20) at a target filter face velocity of $70 \mathrm{~cm} / \mathrm{s}$. Following the FTP, PM sampling began ten seconds after a throttle notch change, and continued for five minutes. Particulate filters were preconditioned and weighed before and after testing, following the FTP. The particulate mass emission rate was computed using the increase of mass on the filters, the volume of dilute exhaust drawn through the filters, and dilution air and raw exhaust flow parameters (fuel and computed air flow). 


\section{Smoke Opacity Test Procedures}

Smoke opacity was measured using a modified Public Health Service (PHS) full-flow opacity meter (smokemeter) mounted above one of the two locomotive exhaust stacks. This smokemeter used standard PHS smokemeter optics and electronics, but was modified to a 20 -inch diameter to accommodate larger exhaust plume diameters. The construction, calibration, and operation of the smokemeter adhered to the FTP.

The smokemeter was aligned with the long axis of the rectangular exhaust stack, resulting in a through-exhaust path length of approximately 14 inches (as determined by the dimensions of the exhaust stack extension). The center of the light beam was positioned $5 \pm 1$ inches above the outlet of the exhaust stack extension. The smokemeter control unit was located in the control building. Voltage output proportional to opacity was recorded on a strip chart, along with engine speed. Smoke opacity was continuously monitored during the EPA Locomotive Test Sequence.

\section{Duty-Cycle Weighting Factors}

Table 3 gives the weighting factors for the two duty cycles applied to the individual notch data points to compute "EPA Line-Haul" and "EPA Switch" duty-cycle weighted composite results. The two EPA cycles are specified in Table B132-1 of $\$ 92.131$ of CFR Title 40, Part 92 of the FTP.

Table 3. Duty Cycles Used to Compute Weighted Composite Emissions

\begin{tabular}{||l|c|c||}
\hline Throttle Notch Setting & EPA Line-Haul Cycle & $\begin{array}{c}\text { EPA Switch } \\
\text { Cycle }\end{array}$ \\
\hline \hline Low Idle & $19.0 \%$ & $29.9 \%$ \\
\hline Idle & $19.0 \%$ & $29.9 \%$ \\
\hline Dynamic Brake & $12.5 \%$ & $0.0 \%$ \\
\hline Notch 1 & $6.5 \%$ & $12.4 \%$ \\
\hline Notch 2 & $6.5 \%$ & $12.3 \%$ \\
\hline Notch 3 & $5.2 \%$ & $5.8 \%$ \\
\hline Notch 4 & $4.4 \%$ & $3.6 \%$ \\
\hline Notch 5 & $3.8 \%$ & $3.6 \%$ \\
\hline Notch 6 & $3.9 \%$ & $1.5 \%$ \\
\hline Notch 7 & $3.0 \%$ & $0.2 \%$ \\
\hline Notch 8 & $16.2 \%$ & $0.8 \%$ \\
\hline TOTAL & $\mathbf{1 0 0 . 0} \%$ & $\mathbf{1 0 0 . 0} \%$ \\
\hline \hline
\end{tabular}




\section{F. Test Sequence}

One problem commonly reported with the use of biodiesel fuels in older engines is fuel filter plugging resulting from the solvent nature of the biodiesel, removing accumulated deposits, gum, and varnish from the fuel system. Filter plugging usually occurs only once, after the initial removal of deposits. In anticipation of this potential problem with the nearly 30 -year locomotive, SwRI ran the locomotive on neat bio-fuel for approximately two hours at rated power, then shut the locomotive engine down with the neat bio-fuel in the fuel system. The next morning, the locomotive was restarted and run for another hour on neat bio-fuel. Although no operational problems were noticed during this fuel system conditioning, as a precautionary measure, the primary and secondary fuel filters on the locomotive were replaced before beginning the fuel-effects testing.

Triplicate tests were run on each of the four test fuels. The test sequence was randomized to minimize potential bias due to ambient temperature, humidity, and barometric pressure changes during the test program. Table 4 gives the test sequence used.

Table 4. Test Sequence

\begin{tabular}{||c|c|c||}
\hline Test Number & Fuel & Run Number \\
\hline \hline 1 & EPA & $1 / 3$ \\
\hline 2 & B20 & $1 / 3$ \\
\hline 3 & C20 & $1 / 3$ \\
\hline 4 & CARB & $1 / 3$ \\
\hline 5 & CARB & $2 / 3$ \\
\hline 6 & B20 & $2 / 3$ \\
\hline 7 & EPA & $2 / 3$ \\
\hline 8 & C20 & $2 / 3$ \\
\hline 9 & C20 & $3 / 3$ \\
\hline 10 & CARB & $3 / 3$ \\
\hline 11 & B20 & $3 / 3$ \\
\hline 12 & EPA & $3 / 3$ \\
\hline \hline
\end{tabular}




\section{TEST RESULTS}

Table 5 summarizes duty-cycle weighted results from each of the triplicate tests performed on the four fuels. Detailed test results are given in Appendices A through F. Table 5 includes both EPA Line-Haul and Switch duty cycle composite brake-specific emissions of $\mathrm{HC}, \mathrm{CO}, \mathrm{NO}_{\mathrm{X}}$, and PM, and AAR-corrected brake-specific fuel consumption. Average results for the triplicate runs are given for each fuel, along with the coefficient of variation (cov). At the bottom of Table 5, the average percentage change between fuels is reported. No formal statistical analysis of the data was performed to establish statistically significant differences.

Observations of the data in Table 5 show that the use of the CARB diesel reduced $\mathrm{NO}_{\mathrm{x}}$ by one percent on the Line-Haul cycle and by two percent on the Switch cycle, compared to the baseline EPA locomotive certification fuel. This level of $\mathrm{NO}_{\mathrm{X}}$ reduction is notably smaller than the four to six percent reduction in the average Line-Haul cycle $\mathrm{NO}_{\mathrm{x}}$ measured by SwRI in three 4,000 hp, turbocharged, EMD 710 engines in a study for CARB, using the same batch of CARB fuel. ${ }^{2}$

B20 fuel increased $\mathrm{NO}_{\mathrm{x}}$ by six percent on the Line-Haul cycle and by five percent on the Switch cycle, compared to the baseline EPA locomotive certification fuel. This $\mathrm{NO}_{\mathrm{x}}$ increase is in the range noted for other diesel engine studies using $\mathrm{B} 20$ fuel. Comparing $\mathrm{C} 20$ to CARB fuel, $\mathrm{NO}_{\mathrm{x}}$ increased by four percent for both cycles. Comparing C20 to the baseline EPA locomotive certification fuel, the $\mathrm{NO}_{\mathrm{X}}$ increase associated with the biodiesel is partially offset with the $\mathrm{NO}_{\mathrm{X}}$ reduction associated with the $\mathrm{CARB}$ fuel, resulting in a $\mathrm{NO}_{\mathrm{X}}$ increase of three percent on the Line-Haul cycle and two percent on the Switch cycle.

PM emissions for this locomotive engine were generally unresponsive to fuel type, with small changes likely due to test-to-test variability. Because PM emissions from two-stroke EMD engines are dominated by lubricating-oil derived components, the influence of fuel type is relatively small. In the CARB locomotive fuel effects study, the soluble organic fraction (SOF) of PM emissions from the turbocharged EMD engines accounted for about 75 percent of total PM. Although no SOF analyses were performed on the PM samples in this project, nonturbocharged EMD engines, like that tested in this project, typically have higher brake-specific lubricating oil consumption than their turbocharged counterparts, suggesting that the SOF of PM would be even higher than observed for the turbocharged engines.

Hydrocarbon emissions also were generally unresponsive to fuel type using the Line-Haul cycle, but some reductions on the Switch cycle were noted with all three fuels compared to the EPA certification fuel, although the changes were likely not be statistically significant. 
Table 5. CSX No 2629 Test Summary

\begin{tabular}{|c|c|c|c|c|c|c|c|c|c|c|}
\hline & \multicolumn{5}{|c|}{ EPA Line-Haul Duty Cycle } & \multicolumn{5}{|c|}{ EPA Switch Duty Cycle } \\
\hline & $\begin{array}{c}\text { HC } \\
\text { g/hp-hr }\end{array}$ & $\begin{array}{c}\text { CO } \\
\text { g/hp-hr }\end{array}$ & $\begin{array}{c}\text { NOx } \\
\text { g/hp-hr }\end{array}$ & \begin{tabular}{|c} 
PM \\
g/hp-hr
\end{tabular} & $\begin{array}{l}\text { AAR Corr. } \\
\text { BSFC } \\
\text { lb/hp-hr }\end{array}$ & $\begin{array}{c}\text { HC } \\
\text { g/hp-hr }\end{array}$ & $\begin{array}{c}\text { CO } \\
\text { g/hp-hr }\end{array}$ & $\begin{array}{c}\text { NOx } \\
\text { g/hp-hr }\end{array}$ & $\begin{array}{c}\text { PM } \\
\text { g/hp-hr }\end{array}$ & $\begin{array}{l}\text { AAR Corr } \\
\text { BSFC } \\
\text { lb/hp-hr }\end{array}$ \\
\hline EPA Cert Baseline \#1/3 & 0.71 & 5.9 & 11.9 & 0.47 & 0.433 & 0.87 & 2.4 & 12.7 & 0.37 & 0.464 \\
\hline EPA Cert Baseline \#2/3 & 0.62 & 5.1 & 12.3 & 0.49 & 0.435 & 0.80 & 2.2 & 12.9 & 0.42 & 0.474 \\
\hline EPA Cert Baseline \#3/3 & 0.58 & 5.1 & 12.9 & 0.44 & 0.434 & 0.78 & 2.1 & 12.9 & 0.36 & 0.460 \\
\hline Average & 0.64 & 5.4 & 12.4 & 0.46 & 0.434 & 0.82 & 2.2 & 12.8 & 0.38 & 0.466 \\
\hline c.o.v. & $10 \%$ & $9 \%$ & $4 \%$ & $5 \%$ & $0 \%$ & $6 \%$ & $7 \%$ & $1 \%$ & $8 \%$ & $2 \%$ \\
\hline CARB \#1/3 & 0.63 & 4.6 & 12.2 & 0.48 & 0.431 & 0.78 & 1.8 & 12.7 & 0.34 & 0.467 \\
\hline CARB \#2/3 & 0.62 & 4.7 & 12.0 & 0.46 & 0.429 & 0.77 & 1.9 & 12.3 & 0.35 & 0.457 \\
\hline CARB \#3/3 & 0.67 & 3.7 & 12.6 & 0.45 & 0.433 & 0.72 & 1.6 & 12.7 & 0.32 & 0.466 \\
\hline Average & 0.64 & 4.3 & 12.3 & 0.46 & 0.431 & 0.76 & 1.8 & 12.5 & 0.34 & 0.463 \\
\hline c.o.v. & $4 \%$ & $13 \%$ & $3 \%$ & $4 \%$ & $0 \%$ & $4 \%$ & $6 \%$ & $2 \%$ & $5 \%$ & $1 \%$ \\
\hline B20 \#1/3 & 0.66 & 5.3 & 12.6 & 0.48 & 0.430 & 0.78 & 2.2 & 13.4 & 0.36 & 0.462 \\
\hline B20 \#2/3 & 0.63 & 4.2 & 13.0 & 0.55 & 0.431 & 0.73 & 2.0 & 13.4 & 0.38 & 0.468 \\
\hline B20 \#3/3 & 0.64 & 3.9 & 13.6 & 0.46 & 0.434 & 0.82 & 1.9 & 13.8 & 0.38 & 0.470 \\
\hline Average & 0.64 & 4.5 & 13.1 & 0.50 & 0.432 & 0.78 & 2.0 & 13.5 & 0.37 & 0.467 \\
\hline c.o.v. & $2 \%$ & $17 \%$ & $3 \%$ & $10 \%$ & $0 \%$ & $6 \%$ & $9 \%$ & $2 \%$ & $3 \%$ & $1 \%$ \\
\hline C20 \#1/3 & 0.63 & 4.2 & 12.9 & 0.49 & 0.434 & 0.75 & 1.9 & 13.1 & 0.36 & 0.468 \\
\hline C20 \#2/3 & 0.63 & 3.9 & 12.8 & 0.48 & 0.431 & 0.80 & 1.8 & 13.3 & 0.39 & 0.473 \\
\hline C20 \#3/3 & 0.67 & 3.9 & 12.8 & 0.48 & 0.432 & 0.73 & 1.8 & 13.1 & 0.37 & 0.467 \\
\hline Average & 0.64 & 4.0 & 12.8 & 0.48 & 0.432 & 0.73 & 1.8 & 13.1 & 0.37 & 0.467 \\
\hline c.o.v. & $4 \%$ & $4 \%$ & $1 \%$ & $2 \%$ & $0 \%$ & $11 \%$ & $3 \%$ & $2 \%$ & $5 \%$ & $1 \%$ \\
\hline \multicolumn{11}{|c|}{ Average Percent Change from Average EPA Cert. Diesel Baseline } \\
\hline CARB vs. EPA Cert. & $1 \%$ & $-19 \%$ & $-1 \%$ & $0 \%$ & $-1 \%$ & $-7 \%$ & $-21 \%$ & $-2 \%$ & $-12 \%$ & $-1 \%$ \\
\hline B20 vs EPA Cert. & $1 \%$ & $-17 \%$ & $6 \%$ & $7 \%$ & $-1 \%$ & $-5 \%$ & $-10 \%$ & $5 \%$ & $-3 \%$ & $0 \%$ \\
\hline C20 vs EPA Cert & $1 \%$ & $-26 \%$ & $3 \%$ & $4 \%$ & $0 \%$ & $-11 \%$ & $-19 \%$ & $2 \%$ & $-5 \%$ & $0 \%$ \\
\hline C20 vs. CARB & $1 \%$ & $-8 \%$ & $4 \%$ & $4 \%$ & $0 \%$ & $-4 \%$ & $2 \%$ & $4 \%$ & $8 \%$ & $1 \%$ \\
\hline C20 vs. B20 & $0 \%$ & $-11 \%$ & $-2 \%$ & $-2 \%$ & $0 \%$ & $-6 \%$ & $-10 \%$ & $-3 \%$ & $-2 \%$ & $0 \%$ \\
\hline
\end{tabular}

Carbon monoxide (CO) emissions were reduced on both cycles for all three fuels compared to the EPA certification fuel, although again, the changes may not be statistically significant beyond test-to-test variability.

Table 5 also includes AAR-corrected brake-specific fuel consumption results. SwRI used published AAR corrections for ambient air temperature, barometric pressure, fuel temperature, fuel specific gravity, and fuel heating value to compute "AAR-corrected" brake-specific fuel 
consumption from observed values. Results given in Table 5 show that there was essentially no change in the AAR-corrected brake-specific fuel consumption results between the four test fuels.

Compared to the baseline EPA certification fuel, locomotive power output was 1.7 percent lower with the CARB fuel, 0.9 percent lower with the B20 fuel, and 2.4 percent lower with the C20 fuel. The power reductions observed are due to the lower volumetric heating value of the fuels (see Table 2), and that fact that the engine load control system on the GP38-2 locomotive essentially sets to a fixed volumetric fuel rate at each notch position.

Smoke opacity from this type of locomotive engine is characteristically very low, in most cases below the five percent threshold of visibility. Smoke test results show that CSX No. 2629 had very low smoke opacity during steady-state conditions, and during throttle notch changes, and that there was no appreciable change in smoke opacity between the four test fuels. 


\section{CONCLUSIONS}

Exhaust emissions and fuel consumption testing was performed on an EMD GP38-2 locomotive operating on a 20-percent by volume biodiesel blended into EPA locomotive certification diesel fuel and into CARB diesel fuel. No operational problems were observed by SwRI on any of the test fuels, and the locomotive engine was able to produce within 1 to 2 percent of full rated power while operating on the biodiesel blends. Long term durability and reliability were not addressed in this study.

The 5 to 6 percent increase in $\mathrm{NO}_{\mathrm{X}}$ emissions with $\mathrm{B} 20$ fuel compared to EPA locomotive certification diesel was consistent with B20 tests performed in other engine test programs. The lack of PM response with any of the test fuels is attributed to the fact that most of the PM emissions from this mode of two-stroke EMD engine are lubricating-oil derived.

The EMD 645E engine is arguably the most likely candidate for use of biodiesel-blended fuels by the freight railroads, as the $645 \mathrm{E}$ engine is used extensively in switcher and road-switcher applications, where they are likely to be centrally fueled, and operated in urban areas. However, a roots-blown (non-turbocharged) EMD 645E locomotive engine may not have been the best choice for demonstrating fuel effects on PM emissions, due to the dominance lubricating oil consumption has on the PM emissions from these engines. 


\section{APPENDIX A}

\section{BASELINE TESTS WITH EPA CERTIFICATION DIESEL FUEL}

FINAL 08.02999 


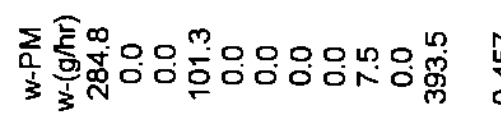

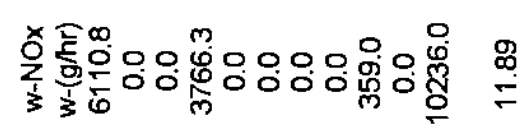

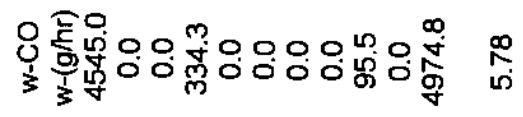

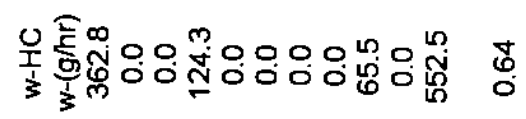

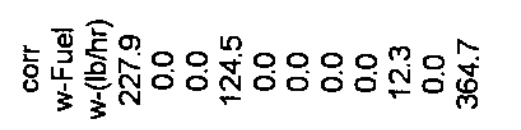

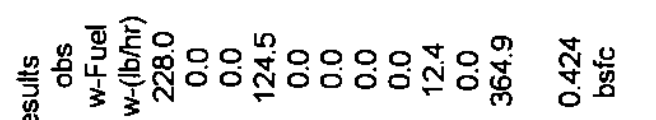


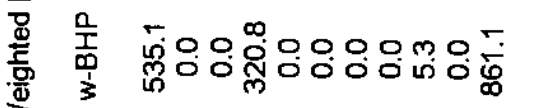

ก/

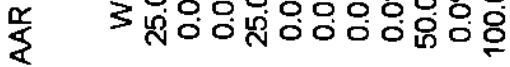

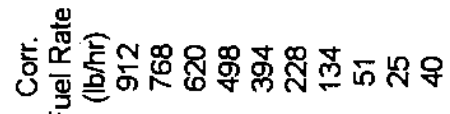

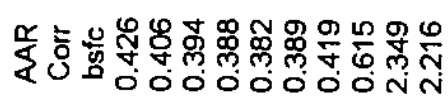

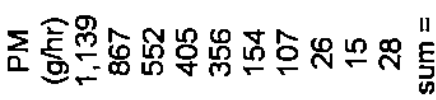

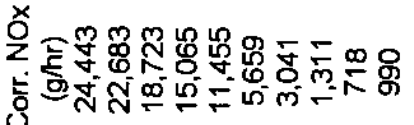

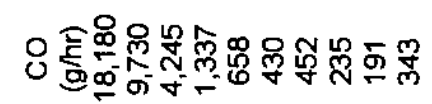

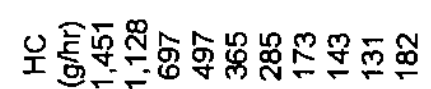

6

$\sum_{i}^{0}$

$\infty$

o

品

芯怘

范

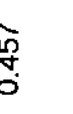

高突

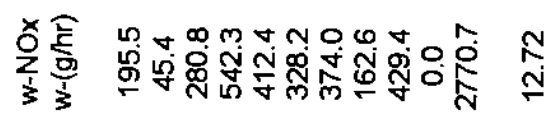

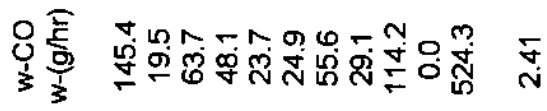

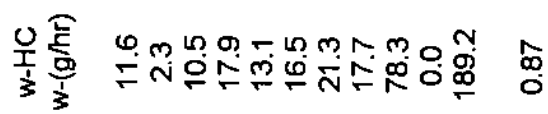

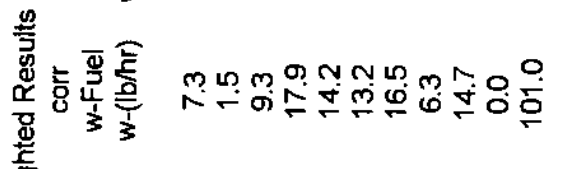

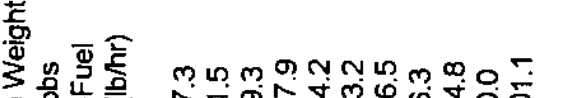

ड़

Uे

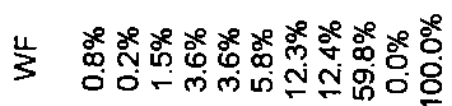

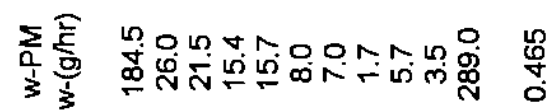

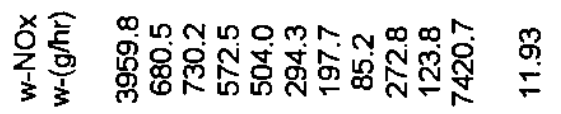

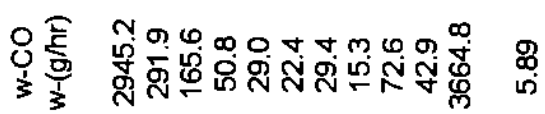

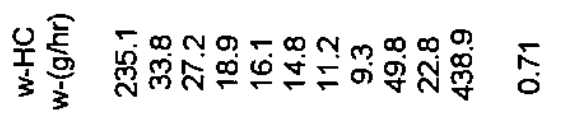

若

吉苛

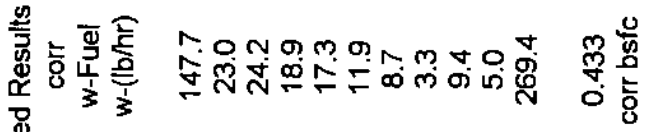

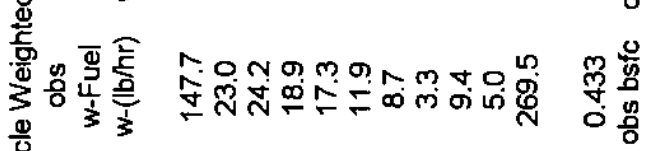

$\frac{10}{0}$

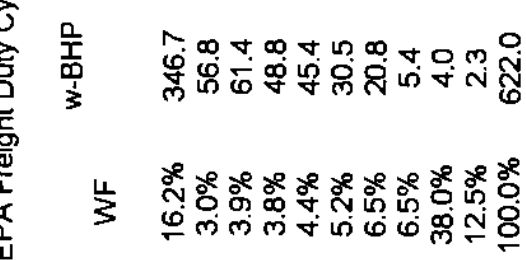

总

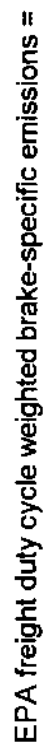




$\begin{array}{ll}\text { LOCOMOTIVE MODEL } & =\text { EMD GP38 } \\ \text { LOCOMOTIVE UNIT \# } & =\text { CSX \#2629 } \\ \text { THROTTLE NOTCH } & =\text { IDLE } \\ \text { TEST DATE } & =10 / 26 / 0 \\ \text { TEST NUMBER } & =1 / 10 \\ \text { SWRI FUEL CODE } & =\text { EM-2834-F } \\ \text { BAROMETER } & =29.21(\mathrm{in} . \mathrm{Hg}) \\ \text { DRY BULB TEMP } & =79 .\left(^{\circ} \mathrm{F}\right) \\ \text { ABS HUMIDITY } & =106.11(\mathrm{GR} / \mathrm{LB})\end{array}$

ENGINE PERFORMANCE SUMMARY:

ACCESSORY HORSEPOWER:

$\begin{array}{rlr}\text { AUXILLARY GENERATOR HP } & =4.0 \\ \text { AIR COMPRESSOR HP } & =4.3 \\ \text { TRACTION MOTOR BLOWER HP } & =1.9 \\ \text { INERTIAL SEPERATOR BLOWER HP } & =.3 \\ \text { RADIATOR FAN HP } & =.0 \\ \text { TOTAL ACCESSORY HP } & =10.5\end{array}$

FUEL RATE (OBS) $=24.8(\mathrm{LB} / \mathrm{HR})$

ALT. VOLTS $=3 . \quad$ ALT. AMPS $=0 . \quad$ ALT. EFF. $=.939$

FLYWHEEL HP $=10.5 \quad$ OBS. SFC $=2.3562 \quad$ AAR CORR. SFC $=2.3491$ (LB/HP-HR)

CALCULATED A/F $=321.54$ (LB DRY AIR/LB FUEL)

EMISSIONS G/HR G/HP.HR CONC.

$\begin{array}{llcc}\text { HC } & 131 . & 12.43 & 75 . \text { PPMC } \\ \text { CO } & 191 . & 18.14 & 55 . \text { PPM } \\ \text { NOX } & 661 . & 62.76 & 115 . \text { PPM (D) } \\ \text { NOX, CORR } & 718 . & 68.24 & \\ \text { C02 } & & 3331 . & .64 \text { PCT } \\ 02 & & 20.03 \text { PCT }\end{array}$

CORRECTION FACTOR SUMMARY:

$\begin{array}{lllll}\text { EPA NOX CF }=1.0874 & \text { NOX-KH } & =1.0714 & \text { NOX-KT } & =1.0000 \\ \text { WET EXH HC CF }=.9892 & \text { AIR TEMP CF }=.9878 & \text { BAROM CF } & =1.0030 \\ \text { FUEL TEMP CF }=.9850 & \text { FUEL S.G. CF }=.9907 & \text { FUEL HHV CF } & =1.0062\end{array}$

PARTICULATE INFORMATION

FILTER PAIR I.D. NUMBER

FILTER PAIR WEIGHT GAIN (mg)

$8364.0 \cdot 122$

1.501

38.667

.039

3.540

.137

15.

1.390 


$\begin{array}{ll}\text { LOCOMOTIVE MODEL } & =\text { EMD GP38 } \\ \text { LOCOMOTIVE UNIT \# } & =\text { CSX \#2629 } \\ \text { THROTTLE NOTCH } & =\text { DB2 } \\ \text { TEST DATE } & =10 / 26 / 0 \\ \text { TEST NUMBER } & =2 / 10 \\ \text { SWRI FUEL CODE } & =\text { EM-2834-F } \\ \text { BAROMETER } & =29.21(\mathrm{in} . \mathrm{Hg}) \\ \text { DRY BULB TEMP } & =79 .\left({ }^{\circ} \mathrm{F}\right) \\ \text { ABS HUMIDITY } & =107.80(\mathrm{GR} / \mathrm{LB})\end{array}$

ENGINE PERFORMANCE SUMMARY:

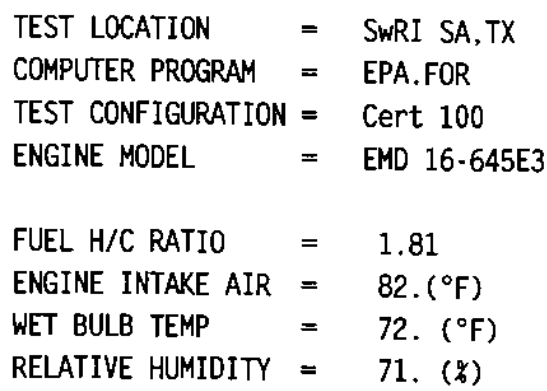

ACCESSORY HORSEPOWER:

$\begin{array}{rlr}\text { AUXILLARY GENERATOR HP } & =4.0 \\ \text { AIR COMPRESSOR HP } & =6.5 \\ \text { TRACTION MOTOR BLOWER HP } & =6.6 \\ \text { INERTIAL SEPERATOR BLOWER HP } & =1.0 \\ \text { RADIATOR FAN HP } & .0 \\ \text { TOTAL ACCESSORY HP } & =18.0\end{array}$

FUEL RATE (OBS) =

40.0 (LB/HR)

ALT. VOLTS $=7$.

ALT. AMPS $=$

0 . ALT. EFF. $=.939$

FLYWHEEL HP

$18.0 \quad$ OBS. $\mathrm{SFC}=2.2174$

CALCULATED A/F $=286.11$ (LB DRY AIR/LB FUEL)

EMISSIONS G/HR G/HP-HR CONC.

$\begin{array}{lccc}\text { HC } & 182 . & 10.07 & 73 . \text { PPMC } \\ \text { CO } & 343 . & 19.04 & 69 . \text { PPM } \\ \text { NOX } & 906 . & 50.25 & 110 . \text { PPM (D) } \\ \text { NOX, CORR } & 990 . & 54.88 & \\ \text { CO2 } & & 3137 . & .72 \text { PCT } \\ \text { O2 } & & & 20.03 \text { PCT }\end{array}$

CORRECTION FACTOR SUMMARY:

$\begin{array}{lllll}\text { EPA NOX CF }=1.0921 & \text { NOX-KH } & =1.0752 & \text { NOX-KT } & =1.0000 \\ \text { WET EXH HC CF }=.9884 & \text { AIR TEMP CF }=.9901 & \text { BAROM CF } & =1.0030 \\ \text { FUEL TEMP CF }=.9835 & \text { FUEL S.G. CF }=.9907 & \text { FUEL HHV CF } & =1.0062\end{array}$

PARTICULATE INFORMATION

-......................

FILTER PAIR I.D. NUMBER

$8365.0-123$

FILTER PAIR WEIGHT GAIN (mg)

1.919

SAMPLE VOLUME (SCf)

38.379

DILUTE PM CONCENTRATION (mg/scf)

.050

3.713

CO2-BASED DILUTION FACTOR

.186

PARTICULATE CONCENTRATION IN RAW EXHAUST (mg/scf)

PARTICULATE MASS EMISSION RATE $(\mathrm{g} / \mathrm{hr})$

28.

BRAKE-SPECIFIC PARTICULATE EMISSION RATE $(\mathrm{g} / \mathrm{hp}-\mathrm{hr})$

1.573 


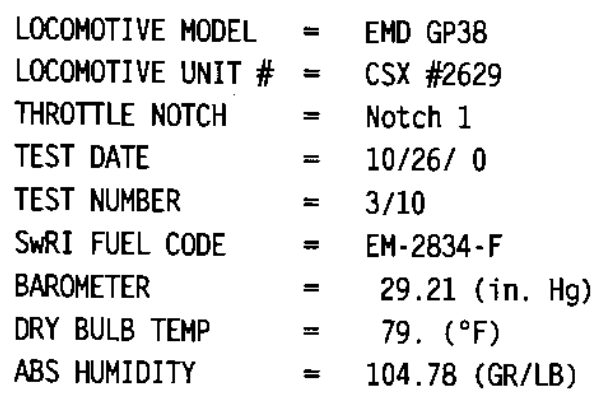

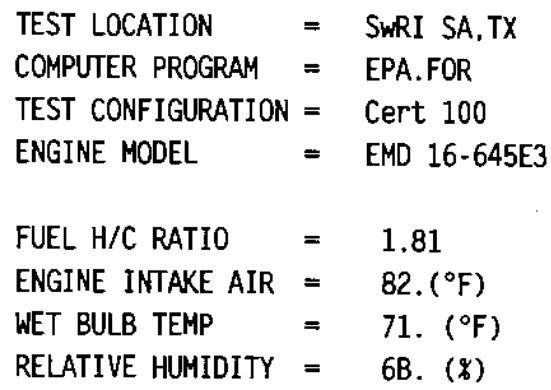

ENGINE PERFORMANCE SUMMARY:

ACCESSORY HORSEPOWER:

$\begin{array}{rlr}\text { AUXILLARY GENERATOR HP } & =4.0 \\ \text { AIR COMPRESSOR HP } & =5.3 \\ \text { TRACTION MOTOR BLOWER HP } & =3.6 \\ \text { INERTIAL SEPERATOR BLOWER HP } & =5 \\ \text { RADIATOR FAN HP } & .5 \\ \text { TOTAL ACCESSORY HP } & =13.4\end{array}$

FUEL RATE (OBS) $=51.0(\mathrm{LB} / \mathrm{HR})$

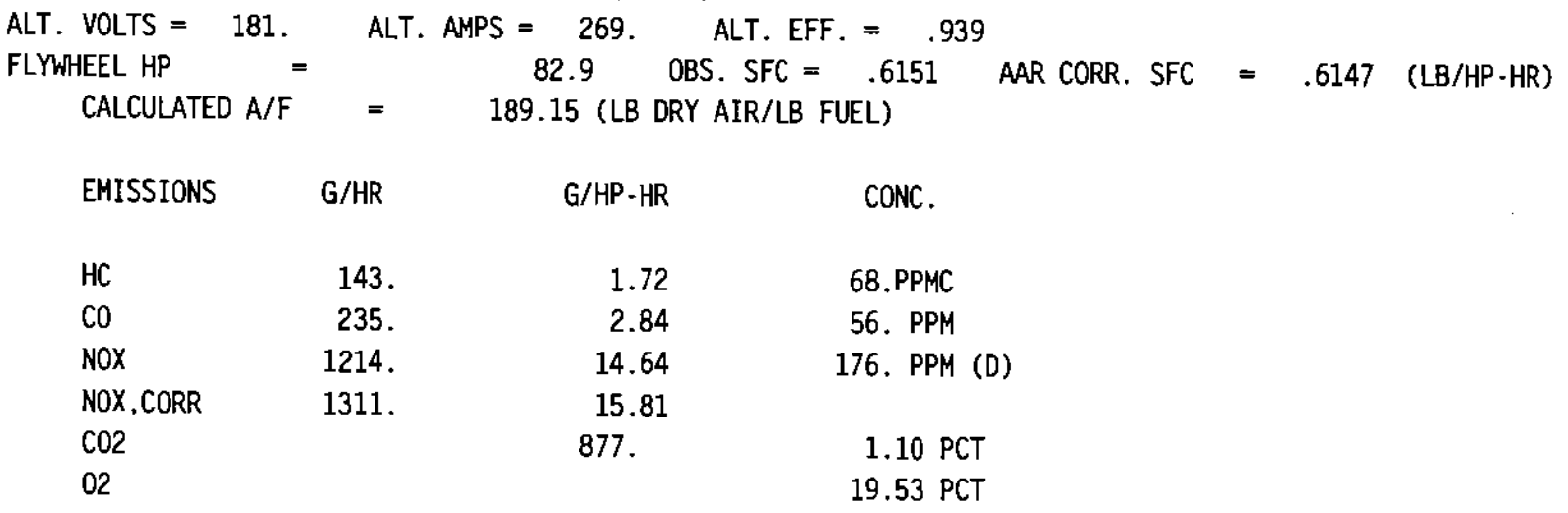

CORRECTION FACTOR SUMMARY:

$\begin{array}{lllll}\text { EPA NOX CF }=1.0804 & \text { NOX-KH } & =1.0657 & \text { NOX-KT } & =1.0000 \\ \text { WET EXH HC CF }=.9852 & \text { AIR TEMP CF }=.9901 & \text { BAROM CF } & =1.0030 \\ \text { FUEL TEMP CF }=.9835 & \text { FUEL S.G. CF }=.9907 & \text { FUEL HHV CF } & =1.0062\end{array}$

PARTICULATE INFORMATION

FILTER PAIR I.D. NUMBER

FILTER PAIR WEIGHT GAIN (mg)

$8366.0-126$

SAMPLE VOLUME (sCf)

1.968

38.547

.051

3.895

CO2-BASED DILUTION FACTOR

.199

PARTICULATE CONCENTRATION IN RAW EXHAUST (mg/scf)

PARTICULATE MASS EMISSION RATE $(\mathrm{g} / \mathrm{hr})$

26.

BRAKE-SPECIFIC PARTICULATE EMISSION RATE $(\mathrm{g} / \mathrm{hp} \cdot \mathrm{hr})$

.310 


$\begin{array}{ll}\text { LOCOMOTIVE MODEL } & =\text { EMD GP38 } \\ \text { LOCOMOTIVE UNIT \# } & \text { CSX \#2629 } \\ \text { THROTTLE NOTCH } & =\text { Notch } 2 \\ \text { TEST DATE } & =10 / 26 / 0 \\ \text { TEST NUMBER } & =4 / 10 \\ \text { SWRI FUEL CODE } & =\text { EM-2834-F } \\ \text { BAROMETER } & =29.20(\text { in. Hg) } \\ \text { DRY BULB TEMP } & =80 .\left({ }^{\circ} \mathrm{F}\right) \\ \text { ABS HUMIDITY } & =104.85(\mathrm{GR} / \mathrm{LB})\end{array}$

\begin{tabular}{|c|c|}
\hline TEST LOCATION & $=$ SWRI SA.TX \\
\hline COMPUTER PROGRAM & $=\quad E P A . F O R$ \\
\hline TEST CONFIGURATIDN & Cert 100 \\
\hline ENGINE MODEL & EMD $16-645 \mathrm{E}$ \\
\hline FUEL $H / C$ RATIO & 1.81 \\
\hline ENGINE INTAKE AIR & $80 .\left({ }^{\circ} \mathrm{F}\right)$ \\
\hline WET BULB TEMP & 72. $\left({ }^{\circ} \mathrm{F}\right)$ \\
\hline RELATIVE HUMIDITY & 66. (\%) \\
\hline
\end{tabular}

ENGINE PERFORMANCE SUMMARY:

ACCESSORY HORSEPOWER:

$\begin{array}{rlr}\text { AUXILLARY GENERATOR HP } & = & 4.0 \\ \text { AIR COMPRESSOR HP } & =6.5 \\ \text { TRACTION MOTOR BLOWER HP } & =6.7 \\ \text { INERTIAL SEPERATOR BLOWER HP } & =1.0 \\ \text { RADIATOR FAN HP } & 3.1 \\ \text { TOTAL ACCESSORY HP } & =21.3\end{array}$

FUEL RATE (OBS) =

$134.0(\mathrm{LB} / \mathrm{HR})$

ALT. VOLTS $=377 . \quad$ ALT. AMPS $=554 . \quad$ ALT. EFF. $=.939$

FLYWHEEL HP $=319.6$ OBS. SFC $=.4192 \quad$ AAR CORR. SFC $=.4193(\mathrm{LB} / \mathrm{HP}-\mathrm{HR})$

CALCULATED A/F $=93.80$ (LB DRY AIR/LB FUEL)

EMISSIONS G/HR G/HP-HR CONC.

$\begin{array}{lrrr}\text { HC } & 173 . & .54 & 63 . \text { PPMC } \\ \text { CO } & 452 . & 1.41 & 83 . \text { PPM } \\ \text { NOX } & 2830 . & 8.85 & 316 . \text { PPM (D) } \\ \text { NOX, CORR } & 3041 . & 9.52 & \\ \text { C02 } & & 601 . & 2.24 \text { PCT } \\ 02 & & & 17.91 \text { PCT }\end{array}$

CORRECTION FACTOR SUMMARY:

$\begin{array}{lllll}\text { EPA NOX CF }=1.0747 & \text { NOX-KH } & =1.0610 & \text { NOX-KT } & =1.0000 \\ \text { WET EXH HC CF }=.9752 & \text { AIR TEMP CF }=.9910 & \text { BAROM CF } & =1.0029 \\ \text { FUEL TEMP CF }=.9835 & \text { FUEL S.G. CF }=.9907 & \text { FUEL HHV CF } & =1.0062\end{array}$

PARTICULATE INFORMATION

FILTER PAIR I.D. NUMBER

$8367.0-127$

FILTER PAIR WEIGHT GAIN (mg)

5.774

SAMPLE VOLUME (SCf)

38.067

DILUTE PM CONCENTRATION (mg/scf)

.152

CO2-BASED DILUTION FACTOR

4.175

PARTICULATE CONCENTRATION IN RAW EXHAUST ( $\mathrm{mg} / \mathrm{scf}$ )

.633

PARTICULATE MASS EMISSION RATE $(\mathrm{g} / \mathrm{hr})$

107.

BRAKE-SPECIFIC PARTICULATE EMISSION RATE $(g / h p-h r)$

.335 


$\begin{array}{ll}\text { LOCOMOTIVE MODEL } & =\text { EMD GP38 } \\ \text { LOCOMOTIVE UNIT \# } & =\text { CSX } \# 2629 \\ \text { THROTLE NOTCH } & =\text { Notch } 3 \\ \text { TEST DATE } & =10 / 26 / 0 \\ \text { TEST NUMBER } & =5 / 10 \\ \text { SWRI FUEL COOE } & =\mathrm{EM}-2834-\mathrm{F} \\ \text { BAROMETER } & =29.20(\mathrm{in} . \mathrm{Hg}) \\ \text { DRY BULB TEMP } & =80 .\left({ }^{\circ} \mathrm{F}\right) \\ \text { ABS HUMIDITY } & =99.70(\mathrm{GR} / \mathrm{LB})\end{array}$

$\begin{array}{ll}\text { TEST LOCATION } & =\text { SWRI SA,TX } \\ \text { COMPUTER PROGRAM } & =\text { EPA.FOR } \\ \text { TEST CONFIGURATION } & =\text { Cert } 100 \\ \text { ENGINE MODEL } & =\text { EMD } 16 \cdot 645 E 3 \\ \text { FUEL H/C RATIO } & =1.81 \\ \text { ENGINE INTAKE AIR } & =78 .\left(^{\circ} \mathrm{F}\right) \\ \text { WET BULB TEMP } & =71 .\left({ }^{\circ} \mathrm{F}\right) \\ \text { RELATIVE HUMIOITY } & =62 .(\%)\end{array}$

ENGINE PERFORMANCE SUMMARY:

ACCESSORY HORSEPOWER:

$\begin{array}{rrr}\text { AUXILLARY GENERATOR HP } & = & 4.0 \\ \text { AIR COMPRESSOR HP } & =8.3 \\ \text { TRACTION MOTOR BLOWER HP } & =14.0 \\ \text { INERTIAL SEPERATOR BLOWER HP } & =2.1 \\ \text { RADIATOR FAN HP } & =6.5 \\ \text { TOTAL ACCESSORY HP } & =35.0\end{array}$

FUEL RATE (OBS) =

ALT. VOLTS $=514$.

FLYWHEEL HP CALCULATED A/F

228.0 (LB/HR)
ALT. AMPS $=751$.
ALT. EFF, $=.939$

586.4 OBS. SFC $=.3888$ AAR CORR. SFC $=.3892(\mathrm{LB} / \mathrm{HP} \cdot \mathrm{HR})$

70.63 (LB DRY AIR/LB FUEL)

$\begin{array}{lrrr}\text { EMISSIONS } & \text { G/HR } & \text { G/HP.HR } & \text { CONC. } \\ \text { HC } & 285 . & .49 & 80 . P P M C \\ \text { CO } & 430 . & .73 & 62 . \text { PPM } \\ \text { NOX } & 5334 . & 9.10 & 466 . \text { PPM (D) } \\ \text { NOX, CORR } & 5659 . & 9.65 & \\ \text { CO2 } & & 558 . & 2.99 \text { PCT } \\ \text { O2 } & & & 16.78 \text { PCT }\end{array}$

CORRECTION FACTOR SUMMARY:

EPA NOX CF $=1.0608$

WET EXH HC CF $=.9690$

NOX-KH $=1.0498$

AIR TEMP CF $=.9919$

NOX-KT $\quad=1.0000$

BAROM CF $\quad=1.0029$

FUEL TEMP CF $=.9835$

FUEL S.G. $C F=.9907$

PARTICULATE INFORMATION

FILTER PAIR I.D. NUMBER

$6716.0-1$

5.279

FILTER PAIR WEIGHT GAIN (mg)

$38.13 \mathrm{~B}$

SAMPLE VOLUME (SCf)

.138

DILUTE PM CONCENTRATION (mg/scf)

5.126

CO2-BASED DILUTION FACTOR

.710

PARTICULATE MASS EMISSION RATE $(\mathrm{g} / \mathrm{hr})$

154.

BRAKE-SPECIFIC PARTICULATE EMISSION RATE $(\mathrm{g} / \mathrm{hp}-\mathrm{hr})$ 


$\begin{array}{ll}\text { LOCOMOTIVE MODEL } & =\text { EMD GP38 } \\ \text { LOCOMOTIVE UNIT \# } & =\text { CSX \#2629 } \\ \text { THROTTLE NOTCH } & =\text { Notch } 4 \\ \text { TEST DATE } & =10 / 26 / 0 \\ \text { TEST NUMBER } & =6 / 10 \\ \text { SWRI FUEL CODE } & =\text { EM-2834-F } \\ \text { BAROMETER } & =29.20(\text { in. Hg) } \\ \text { DRY BULB TEMP } & =81 .\left({ }^{\circ} \mathrm{F}\right) \\ \text { ABS HUMIDITY } & =103.47(\mathrm{GR} / \mathrm{LB})\end{array}$

TEST LOCATION = SWRI SA, TX COMPUTER PROGRAM $=$ EPA.FOR TEST CONFIGURATION $=$ Cert 100 ENGINE MODEL = EMD 16-645E3

FUEL H/C RATIO $=1.81$ ENGINE INTAKE AIR $=80 .\left({ }^{\circ} \mathrm{F}\right)$ WET BULB TEMP $=72 .\left({ }^{\circ} \mathrm{F}\right)$ RELATIVE HUMIDITY $=64 .(\%)$

ENGINE PERFORMANCE SUMMARY:

ACCESSORY HORSEPOWER:

$\begin{array}{rlr}\text { AUXILLARY GENERATOR HP } & = & 4.0 \\ \text { AIR COMPRESSOR HP } & = & 9.6 \\ \text { TRACTION MOTOR BLOWER HP } & = & 21.2 \\ \text { INERTIAL SEPERATOR BLOWER HP } & =3.2 \\ \text { RADIATOR FAN HP } & =9.8 \\ \text { TOTAL ACCESSORY HP } & =47.8\end{array}$

FUEL RATE (OBS) =

394.0 (LB/HR)

ALT. VOLTS $=690$. ALT. AMPS $=998$.

ALT. EFF. $=.939$

FLYWHEEL HP =

CALCULATED A/F

1031.5

OBS. $\mathrm{SFC}=.3820$

46.28 (LB DRY AIR/LB FUEL)

EMISSIONS

G/HR

G/HP-HR

CONC.

$\mathrm{HC}$

$\mathrm{CO}$

365.

658.

.35

90. PPMC

NOX 10705.

10.38

84. PPM

NOX, CORR

11455.

11.10

$\mathrm{CO} 2$

549.

832. PPM (D)

02

4.60 PCT

14.65 PCT

CORRECTION FACTOR SUMMARY:

EPA NOX CF $=1.0701$

WET EXH HC CF $=.9553$

FUEL TEMP CF $=.9830$

AIR TEMP CF $=.9910$

FUEL S.G. CF $=.9907$

$\begin{array}{ll}\text { NOX-KT } & =1.0000 \\ \text { BAROM CF } & =1.0029 \\ \text { FUEL HHY CF } & =1.0062\end{array}$

NOX-KT $=1.0000$

FUEL HHV CF $=1.0062$

PARTICULATE INFORMATION

FILTER PAIR I.D. NUMBER

6717.0-2

9.043

FILTER PAIR WEIGHT GAIN (mg)

38.158

DILUTE PM CONCENTRATION $(\mathrm{mg} / \mathrm{scf})$

.237

6.051

1.434

PARTICULATE CONCENTRATION IN RAW EXHAUST (mg/scf)

356.

BRAKE-SPECIFIC PARTICULATE EMISSION RATE $(\mathrm{g} / \mathrm{hp}-\mathrm{hr})$ 
LOCOMOTIVE EMISSIONS SWRI PROJECT NO. 08-2999-001

\begin{tabular}{|c|c|c|c|}
\hline LOCOMOTIVE MODEL & EMD GP38 & TEST LOCATION & $=$ SWRI SA, TX \\
\hline LOCOMOTIVE UNIT \# & $\operatorname{cs} \# \# 2629$ & COMPUTER PROGRAM & EPA.FOR \\
\hline THROTTLE NOTCH & Notch 5 & TEST CONFIGURATION & Cert 100 \\
\hline TEST DATE & $10 / 26 / 0$ & ENGINE MODEL & EMD 16-645E3 \\
\hline TEST NUMBER & $7 / 10$ & & \\
\hline SWRI FUEL CODE & $=E M-2834-F$ & FUEL H/C RATIO & 1.81 \\
\hline 8AROMETER & $=29.20($ in. $\mathrm{Hg})$ & ENGINE INTAKE AIR & $80 .\left({ }^{\circ} \mathrm{F}\right)$ \\
\hline DRY BULB TEMP & $=82 .\left({ }^{\circ} \mathrm{F}\right)$ & WET BULB TEMP & 71. $\left({ }^{\circ} \mathrm{F}\right)$ \\
\hline ABS HUMIDITY & $=101.52(\mathrm{GR} / \mathrm{LB})$ & RELATIVE HUMIDITY & 61. $(x)$ \\
\hline
\end{tabular}

ENGINE PERFORMANCE SUMMARY:

ACCESSORY HORSEPOWER:

AUXILLARY GENERATOR HP $=4.0$

AIR COMPRESSOR HP $=10.8$

TRACTION MOTOR BLOWER HP $=30.4$

INERTIAL SEPERATOR BLOWER HP $=4.6$

RADIATOR FAN HP $\quad=28.1$

TOTAL ACCESSORY HP $=77.8$

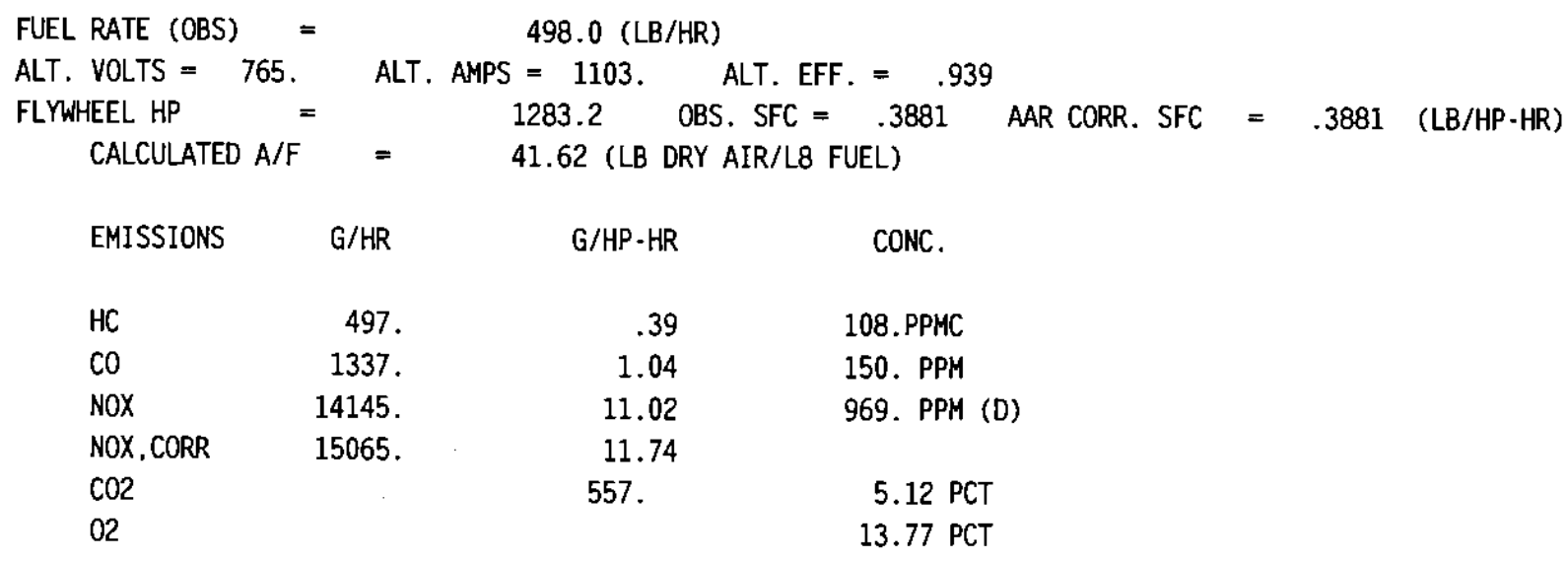

CORRECTION FACTOR SUMMARY:

$\begin{array}{lllll}\text { EPA NOX CF }=1.0651 & \text { NOX-KH } & =1.0532 & \text { NOX-KT } & =1.0000 \\ \text { WET EXH HC CF }=.9511 & \text { AIR TEMP CF }=.9910 & \text { BAROM CF } & =1.0029 \\ \text { FUEL TEMP CF }=.9850 & \text { FUEL S.G. CF }=.9907 & \text { FUEL HHV CF } & =1.0062\end{array}$

PARTICULATE INFORMATION

FILTER PAIR I.D. NUMBER

$6718.0-3$

8.095

FILTER PAIR WEIGHT GAIN (mg)

37.614

DILUTE PM CONCENTRATION (mg/scf) .215

CO2 - BASED DILUTION FACTOR

6.665

1.434

PARTICULATE CONCENTRATION IN RAW EXHAUST (mg/scf)

PARTICULATE MASS EMISSION RATE $(\mathrm{g} / \mathrm{hr})$

405.

BRAKE-SPECIFIC PARTICULATE EMISSION RATE $(g / h p-h r)$ 
LOCOMOTIVE EMISSIONS

$\begin{array}{ll}\text { LOCOMOTIVE MODEL } & =\text { EMD GP38 } \\ \text { LOCOMOTIVE UNIT \# } & =\text { CSX \#2629 } \\ \text { THROTLE NOTCH } & =\text { Notch } 6 \\ \text { TEST DATE } & =10 / 26 / 0 \\ \text { TEST NUMBER } & =8 / 10 \\ \text { SWRI FUEL CODE } & =\text { EM-2834-F } \\ \text { BAROMETER } & =29.19(\mathrm{in} . \mathrm{Hg}) \\ \text { DRY BULB TEMP } & =81 .\left(^{\circ} \mathrm{F}\right) \\ \text { ABS HUMIDITY } & =102.46(\mathrm{GR} / \mathrm{LB})\end{array}$

ENGINE PERFORMANCE SUMMARY:

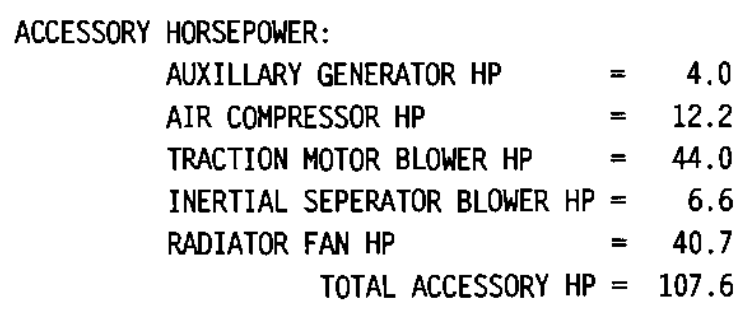

CORRECTION FACTOR SUMMARY:

$\begin{array}{lllll}\text { EPA NOX CF }=1.0674 & \text { NOX-KH } & =1.0551 & \text { NOX-KT } & =1.0000 \\ \text { WET EXH HC CF }=.9461 & \text { AIR TEMP CF }=.9905 & \text { BAROM CF } & =1.0029 \\ \text { FUEL TEMP CF }=.9845 & \text { FUEL S.G. CF }=.9907 & \text { FUEL HHV CF } & =1.0062\end{array}$

PARTICULATE INFORMATION

PARTICULA.................

FILTER PAIR I.D. NUMBER

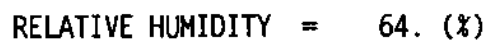

FILTER PAIR WEIGHT GAIN (mg)

$6719.0-4$
8.056
37.261
.216
8.088
1.749
552.

SAMPLE VOLUME (sCf)

DILUTE PM CONCENTRATION (mg/scf)

CO2-BASED DILUTION FACTOR

PARTICULATE CONCENTRATION IN RAW EXHAUST ( $\mathrm{mg} / \mathrm{scf}$ )

PARTICULATE MASS EMISSION RATE $(g / h r)$

BRAKE-SPECIFIC PARTICULATE EMISSION RATE $(\mathrm{g} / \mathrm{hp}-\mathrm{hr})$

.350 


$\begin{array}{ll}\text { LOCOMOTIVE MODEL } & =\text { EMD GP38 } \\ \text { LOCOMOTIVE UNIT \# } & =\text { CSX \#2629 } \\ \text { THROTTLE NOTCH } & =\text { Notch } 7 \\ \text { TEST DATE } & =10 / 26 / 0 \\ \text { TEST NUMBER } & =9 / 10 \\ \text { SWRI FUEL CODE } & =\text { EM }-2834-\mathrm{F} \\ \text { BAROMETER } & =29.19(\mathrm{in} . \mathrm{Hg}) \\ \text { DRY BULB TEMP } & =81 .\left(^{\circ} \mathrm{F}\right) \\ \text { ABS HUMIDITY } & =100.27(\mathrm{GR} / \mathrm{LB})\end{array}$

ENGINE PERFORMANCE SUMMARY:

ACCESSORY HORSEPOWER:

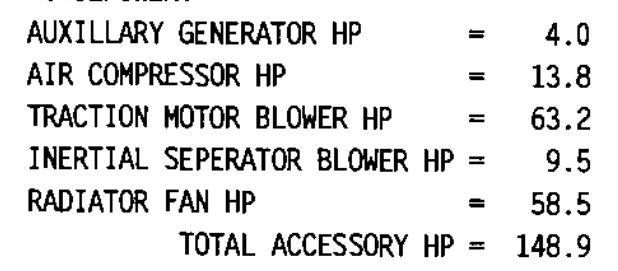

$\begin{array}{rrr}\text { AUXILLARY GENERATOR HP } & = & 4.0 \\ \text { AIR COMPRESSOR HP } & =13.8 \\ \text { TRACTION MOTOR BLOWER HP } & =63.2 \\ \text { INERTIAL SEPERATOR BLOWER HP } & =9.5 \\ \text { RADIATOR FAN HP } & =58.5 \\ \text { TOTAL ACCESSORY HP } & =148.9\end{array}$

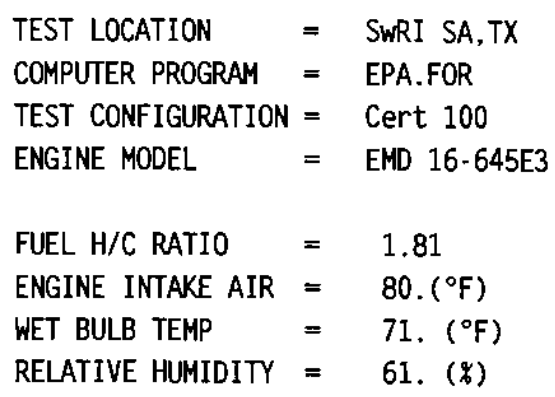




$\begin{array}{ll}\text { LOCOMOTIVE MODEL } & =\text { EMD GP38 } \\ \text { LOCOMOTIVE UNIT \# } & =\text { CSX \#2629 } \\ \text { THROTILE NOTCH } & =\text { Notch } 8 \\ \text { TEST DATE } & =10 / 26 / 0 \\ \text { TEST NUMBER } & =10 / 10 \\ \text { SWRI FUEL CODE } & =\text { EM-2834-F } \\ \text { BAROMETER } & =29.19(\text { in. Hg) } \\ \text { DRY BULB TEMP } & =B 1 .\left({ }^{\circ} \mathrm{F}\right) \\ \text { ABS HUMIDITY } & =102.01(\mathrm{GR} / \mathrm{LB})\end{array}$

$\begin{array}{ll}\text { TEST LOCATION } & =\text { SWRI SA, TX } \\ \text { COMPUTER PROGRAM } & =\text { EPA.FOR } \\ \text { TEST CONFIGURATION } & =\text { Cert } 100 \\ \text { ENGINE MODEL } & =\text { EMD } 16-645 E 3 \\ \text { FUEL H/C RATIO } & =1.81 \\ \text { ENGINE INTAKE AIR } & =81 .\left(^{\circ} \mathrm{F}\right) \\ \text { WET BULB TEMP } & =71 .\left({ }^{\circ} \mathrm{F}\right) \\ \text { RELATIVE HUMIDITY } & =62 .(\%)\end{array}$

ENGINE PERFORMANCE SUMMARY:

ACCESSORY HORSEPOWER:

AUXILLARY GENERATOR HP $\quad=4.0$

AIR COMPRESSOR HP $\quad=15.1$

TRACTION MOTOR BLOWER HP $=83.4$

INERTIAL SEPERATOR BLOWER HP $=12.5$

RADIATOR FAN HP

$=77.2$

TOTAL ACCESSORY HP $=192.2$

FUEL RATE (OBS) = $912.0(\mathrm{LB} / \mathrm{HR})$

ALT. VOLTS $=981 . \quad$ ALT. AMPS $=1390 . \quad$ ALT. EFF. $=.939$

FLYWHEEL HP $=2140.2$ OBS. SFC $=.4261$ AAR CORR. SFC $=.4259(\mathrm{LB} / \mathrm{HP} \cdot \mathrm{HR})$

CALCULATED A/F $=30.74$ (LB DRY AIR/LB FUEL)

EMISSIONS G/HR G/HP-HR CONC.

$\begin{array}{lrrr}\text { HC } & 1451 . & .68 & 230 . \text { PPMC } \\ \text { CO } & 18180 . & 8.49 & 1524 . \text { PPM } \\ \text { NOX } & 22924 . & 10.71 & 1170 . \text { PPM (D) } \\ \text { NOX, CORR } & 24443 . & 11.42 & \\ \text { C02 } & & 599 . & 6.84 \text { PCT } \\ 02 & & & 11.52 \text { PCT }\end{array}$

CORRECTION FACTOR SUMMARY:

$\begin{array}{lllll}\text { EPA NOX CF }=1.0663 & \text { NOX-KH } & =1.0542 & \text { NOX-KT } & =1.0000 \\ \text { WET EXH HC CF }=.9364 & \text { AIR TEMP CF }=.9905 & \text { BAROM CF } & =1.0029 \\ \text { FUEL TEMP CF }=.9860 & \text { FUEL S.G. CF }=.9907 & \text { FUEL HHV CF } & =1.0062\end{array}$

PARTICULATE INFORMATION

FILTER PAIR I.D. NUMBER

$6721.0-6$

14.368

FILTER PAIR WEIGHT GAIN (mg)

34.970

DILUTE PM CONCENTRATION (mg/scf)

.411

CO2-BASED DILUTION FACTOR

7.197

PARTICULATE CONCENTRATION IN RAW EXHAUST $(\mathrm{mg} / \mathrm{scf}$ )

2.957

PARTICULATE MASS EMISSION RATE $(\mathrm{g} / \mathrm{hr})$

1139.

BRAKE-SPECIFIC PARTICULATE EMISSION RATE $(\mathrm{g} / \mathrm{hp}-\mathrm{hr})$

.532 


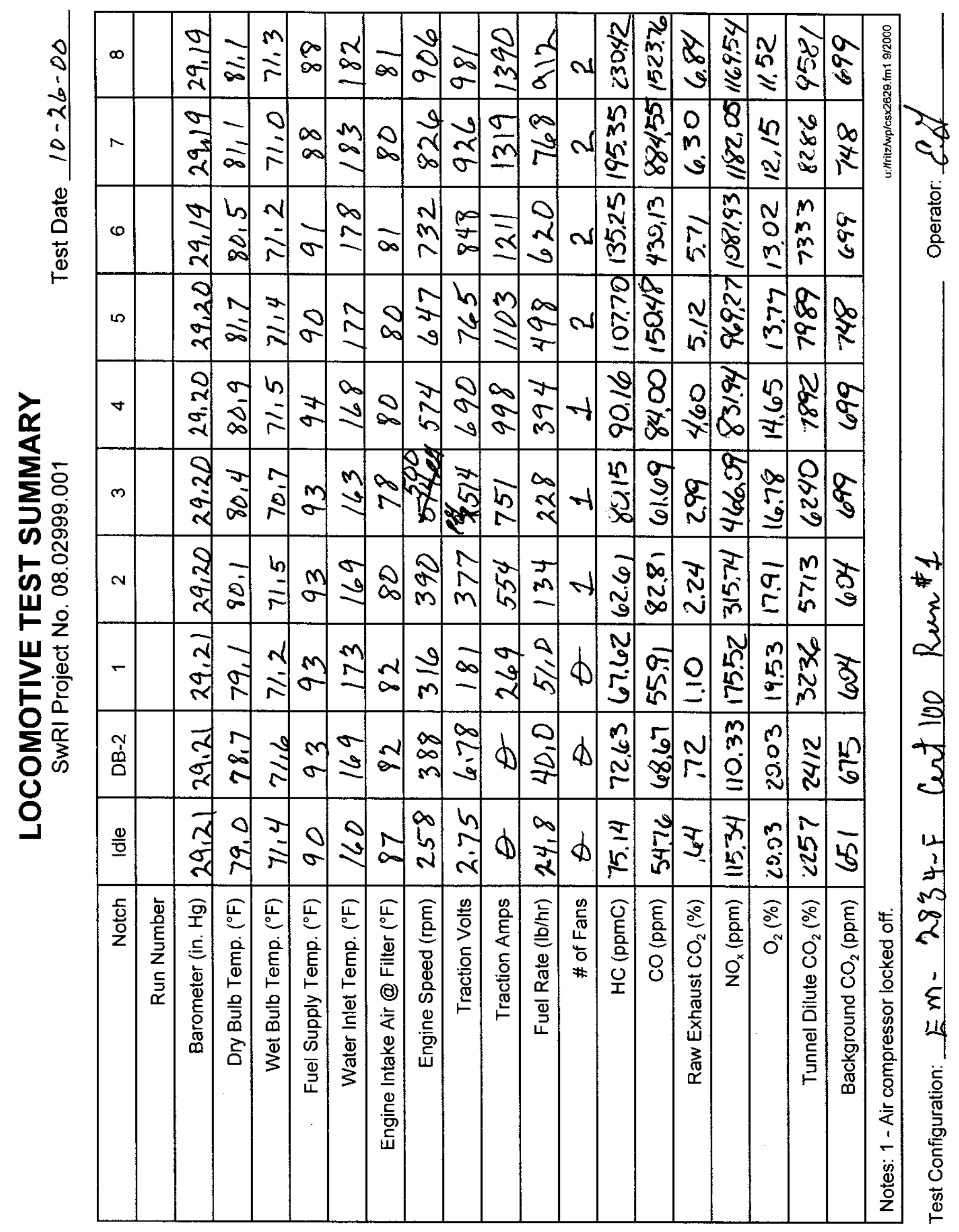




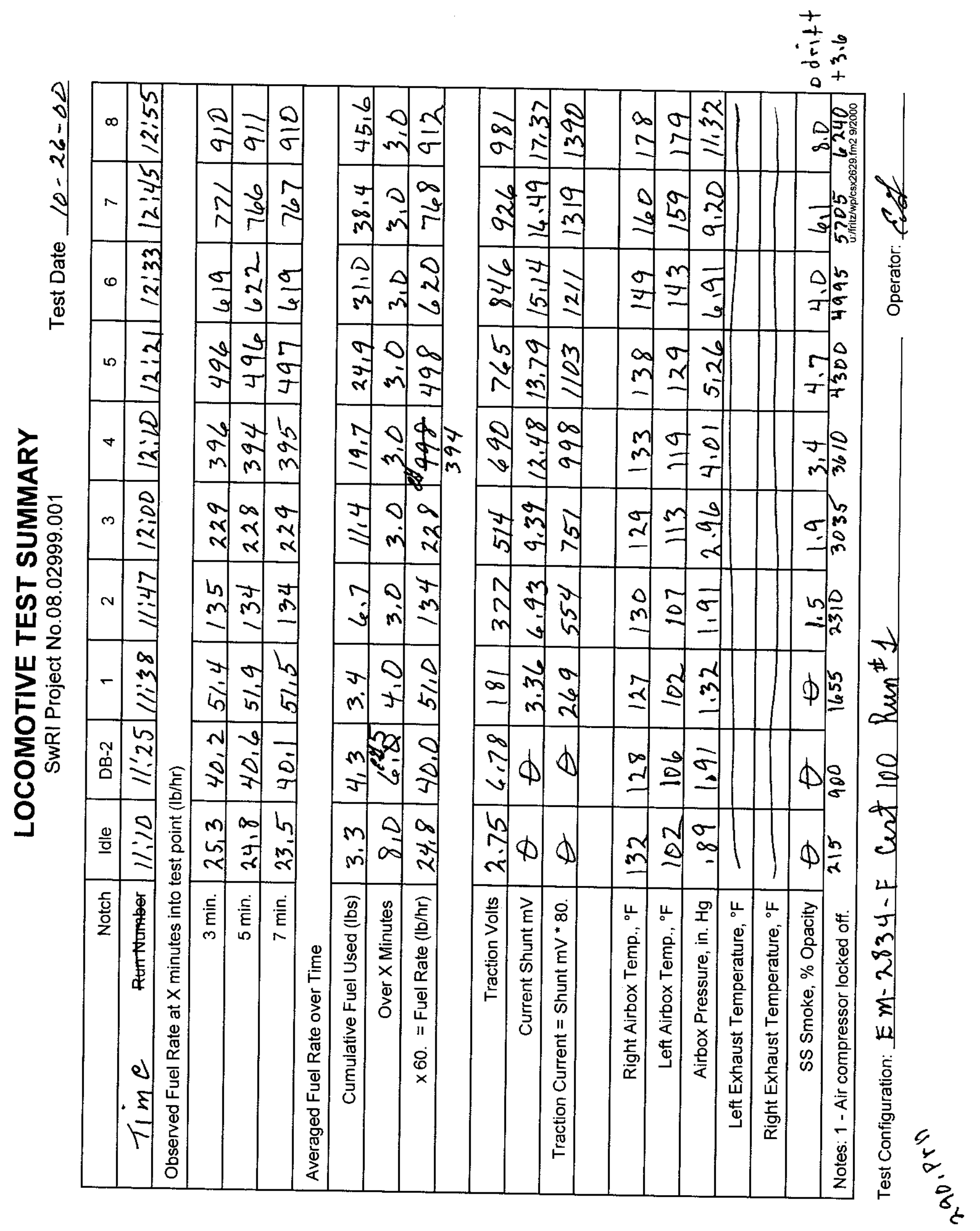




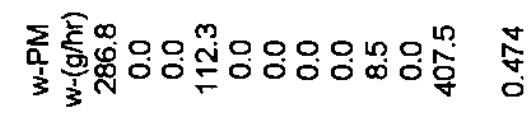

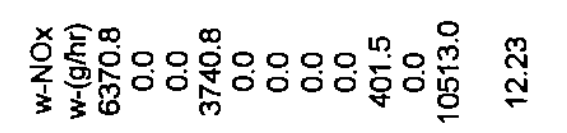

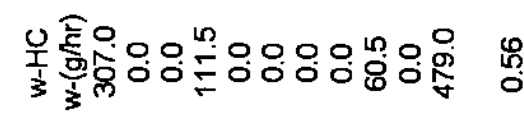

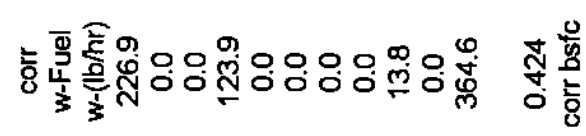

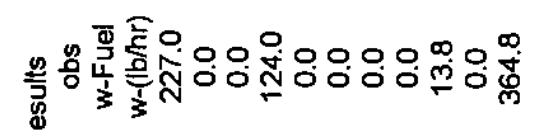

递

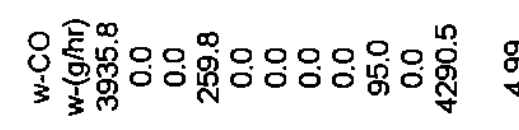

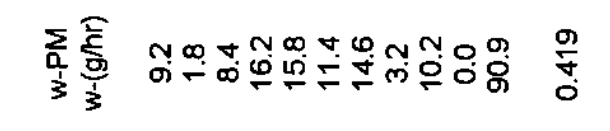

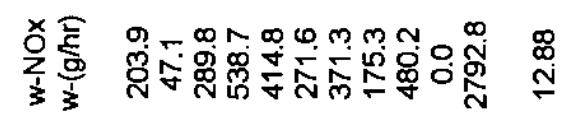

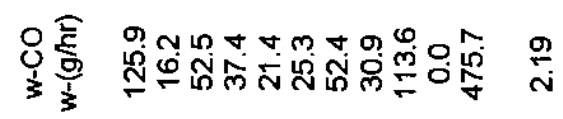

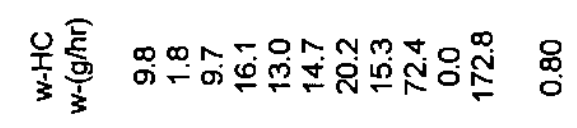

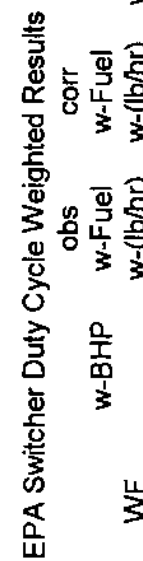

ํํำ

N十⿻上丨

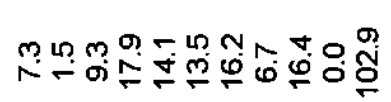

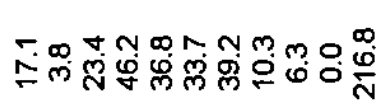

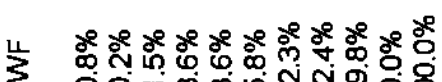

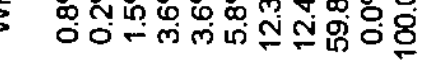

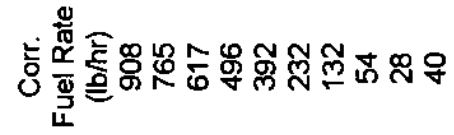

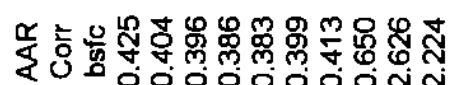

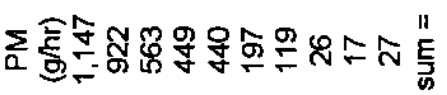

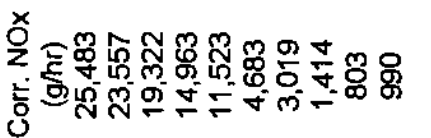

㐫

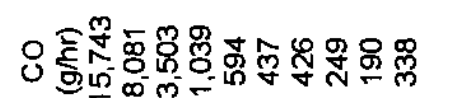

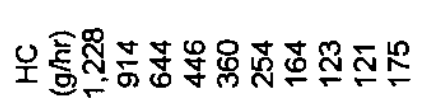

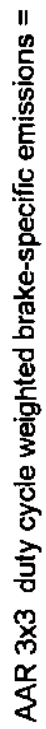

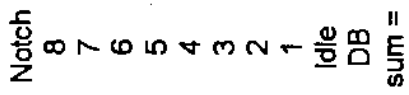

竎

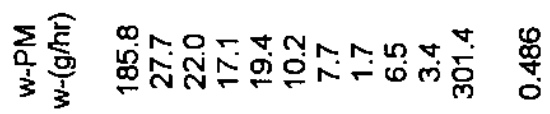

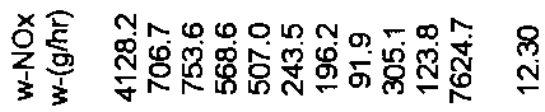

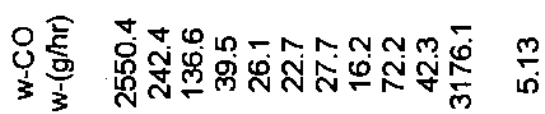

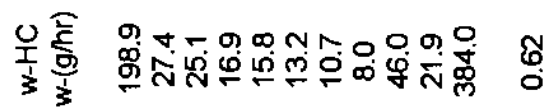

莳落

号贻

娄

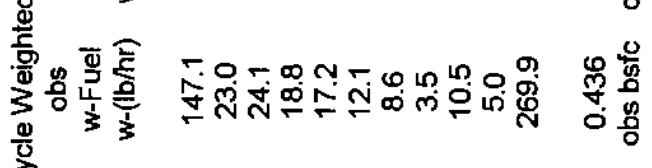

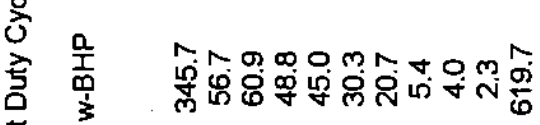

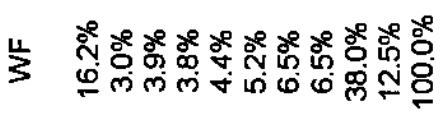

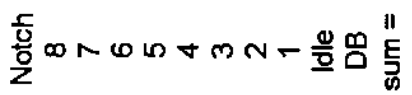

䆛 एᄂ

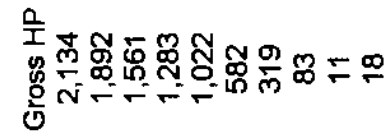

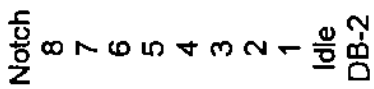




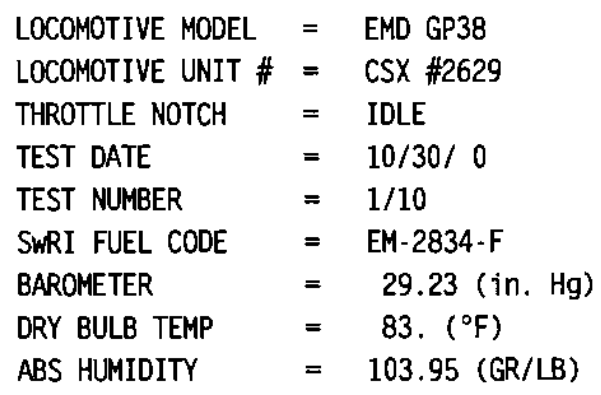

ENGINE PERFORMANCE SUMMARY:

ACCESSORY HORSEPOWER:

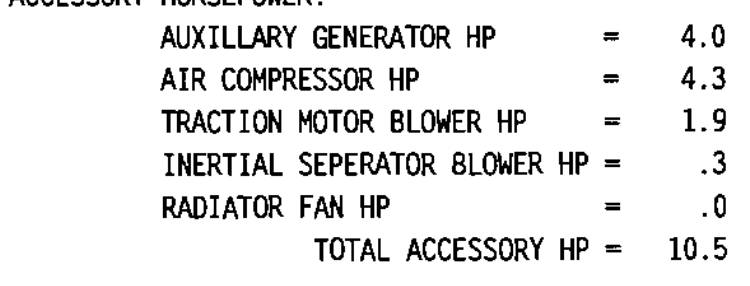

$\begin{array}{rlr}\text { AUXILLARY GENERATOR HP } & = & 4.0 \\ \text { AIR COMPRESSOR HP } & = & 4.3 \\ \text { TRACTION MOTOR BLOWER HP } & =1.9 \\ \text { INERTIAL SEPERATOR BLOWER HP } & = \\ \text { RADIATOR FAN HP } & .3 \\ \text { TOTAL ACCESSORY HP } & =10.5\end{array}$

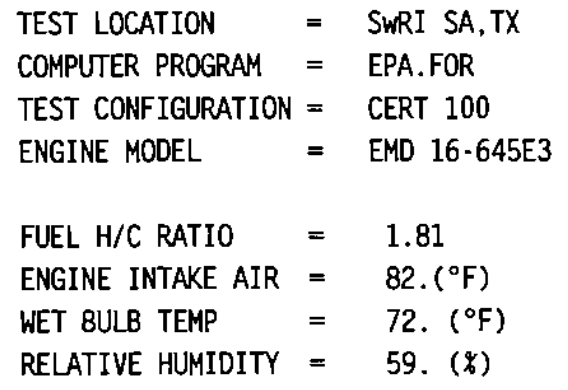




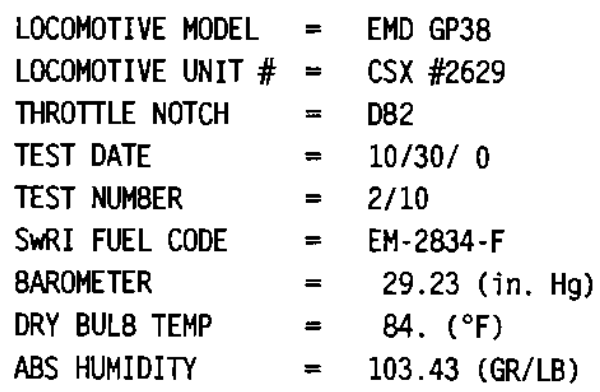

ENGINE PERFORMANCE SUMMARY:

ACCESSORY HORSEPOWER:

$\begin{array}{rlr}\text { AUXILLARY GENERATOR HP } & =4.0 \\ \text { AIR COMPRESSOR HP } & =6.5 \\ \text { TRACTION MOTOR BLOWER HP } & =6.5 \\ \text { INERTIAL SEPERATOR BLOWER HP } & =1.0 \\ \text { RADIATOR FAN HP } & .0 \\ \text { TOTAL ACCESSORY HP } & =18.0\end{array}$

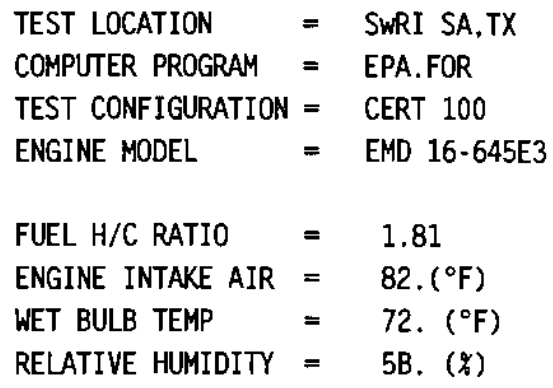

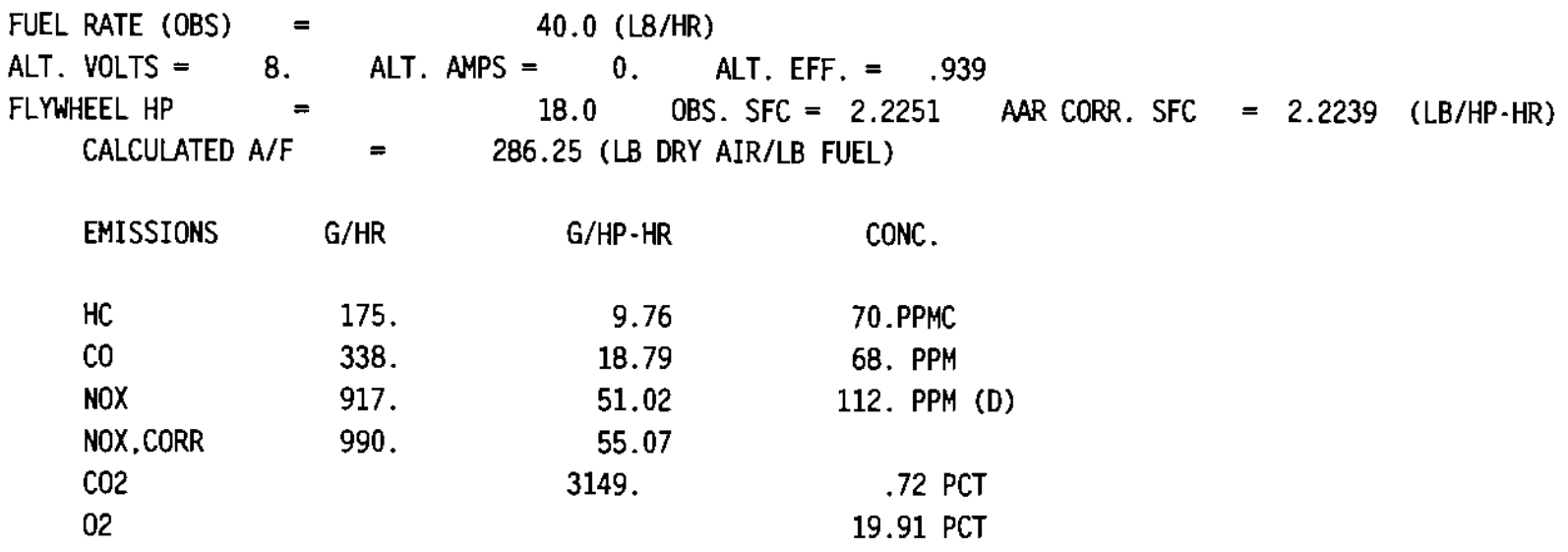

CORRECTION FACTOR SUMMARY:

$\begin{array}{lllll}\text { EPA NOX CF }=1.0793 & \text { NOX-KH } & =1.0648 & \text { NOX-KT } & =1.0000 \\ \text { WET EXH HC CF }=.9886 & \text { AIR TEMP CF }=.9901 & \text { BAROM CF } & =1.0032 \\ \text { FUEL TEMP CF }=.9830 & \text { FUEL S.G. CF }=.9907 & \text { FUEL HHV CF } & =1.0062\end{array}$

PARTICULATE INFORMATION

FILTER PAIR I.D. NUMBER

6773.0-58

1.716

38.626

.044

3.904

.173

27.

1.475 


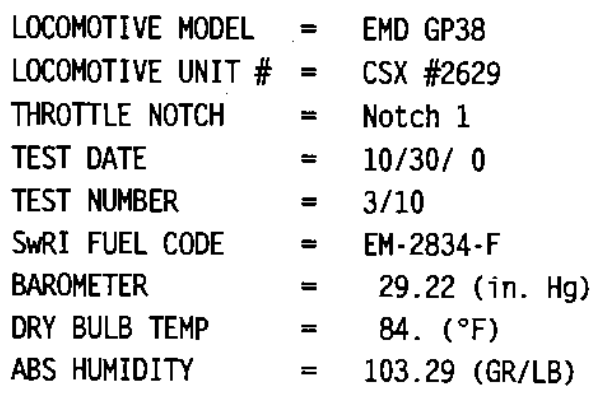

ENGINE PERFORMANCE SUMMARY:

ACCESSORY HORSEPOWER:

$\begin{array}{rlr}\text { AUXILLARY GENERATOR HP } & = & 4.0 \\ \text { AIR COMPRESSOR HP } & =5.3 \\ \text { TRACTION MOTOR BLONER HP } & =3.5 \\ \text { INERTIAL SEPERATOR BLOWER HP } & =.5 \\ \text { RADIATOR FAN HP } & =0 \\ \text { TOTAL ACCESSORY HP } & =13.3\end{array}$

FUEL RATE (OBS)

54.0 (LB/HR)

ALT. VOLTS $=182$.

ALT. AMPS $=268$

ALT. EFF. $=.939$

FLYWHEEL HP =

B3.0 OBS. SFC $=.650$

CALCULATED A/F 189.37 (LB DRY AIR/LB FUEL)

$\begin{array}{lrrr}\text { EMISSIONS } & \text { G/HR } & \text { G/HP.HR } & \text { CONC. } \\ \text { HC } & 123 . & 1.49 & 55 . P P M C \\ \text { CO } & 249 . & 3.01 & 56 . \text { PPH } \\ \text { NOX } & 1314 . & 15.83 & 179 . \text { PPH (0) } \\ \text { NOX.CORR } & 1414 . & 17.04 & \\ \text { C02 } & & 929 . & 1.10 \text { PCT } \\ 02 & & 19.41 \text { PCT }\end{array}$

CORRECTION FACTOR SUMMARY:

EPA NOX CF $=1.0762$

WET EXH HC CF $=.9852$

NOX-KH $=1.0623$

AIR TEMP CF $=.9892$

FUEL TEMP CF $=.9820$

FUEL S.G. CF $=.9907$

PARTICULATE INFORMATION

FILTER PAIR I.D. NUMBER

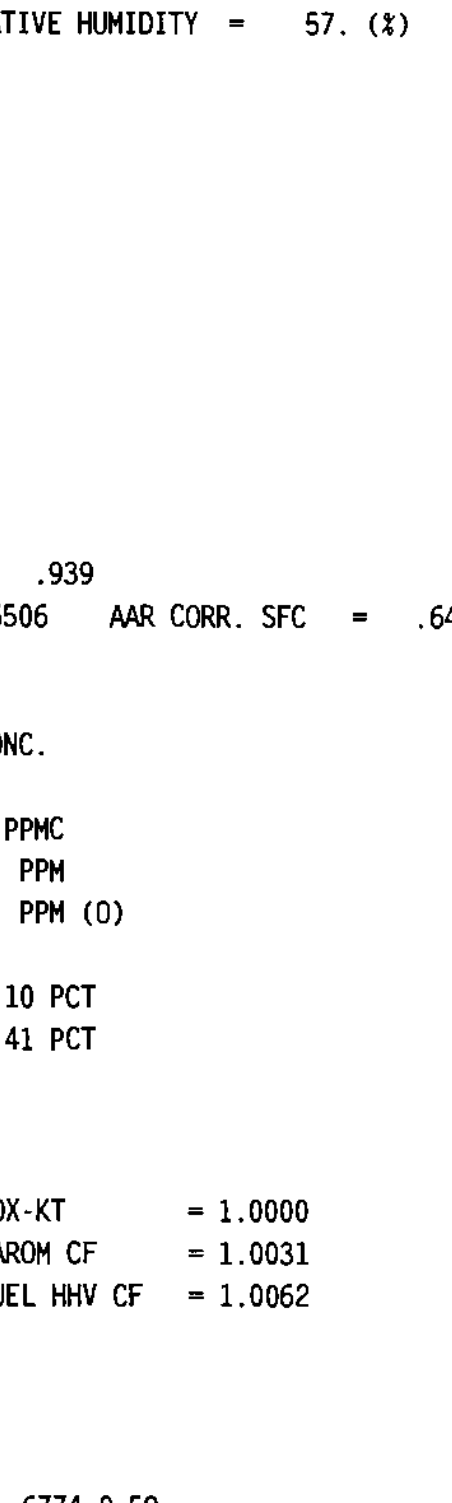

NOX-KT $\quad=1.0000$

BAROM CF $\quad=1.0031$

FUEL HHV CF $=1.0062$

FILTER PAIR WEIGHT GAIN (mg)

$6774.0-59$

1.780

38.555

DILUTE PM CONCENTRATION (mg/scf) $\quad .046$

CO2-BASED DILUTION FACTOR $\quad 4.130$

PARTICULATE CONCENTRATION IN RAW EXHAUST (mg/scf) 191

PARTICULATE MASS EMISSION RATE $(\mathrm{g} / \mathrm{hr})$

26.

BRAKE-SPECIFIC PARTICULATE EMISSION RATE $(\mathrm{g} / \mathrm{hp}-\mathrm{hr})$ 


$\begin{array}{ll}\text { LOCOMOTIVE MODEL } & =\text { EMD GP38 } \\ \text { LOCOMOTIVE UNIT \# } & =\text { CSX \#2629 } \\ \text { THROTILE NOTCH } & =\text { Notch } 2 \\ \text { TEST DATE } & =10 / 30 / 0 \\ \text { TEST NUMBER } & =4 / 10 \\ \text { SWRI FUEL CODE } & =\text { EM-2834-F } \\ \text { BAROMETER } & =29.22(\text { in. Hg) } \\ \text { DRY BULB TEMP } & =86 .\left({ }^{\circ} \mathrm{F}\right) \\ \text { ABS HUMIDITY } & =101.40(\mathrm{GR} / \mathrm{LB})\end{array}$

ENGINE PERFORMANCE SUMMARY:

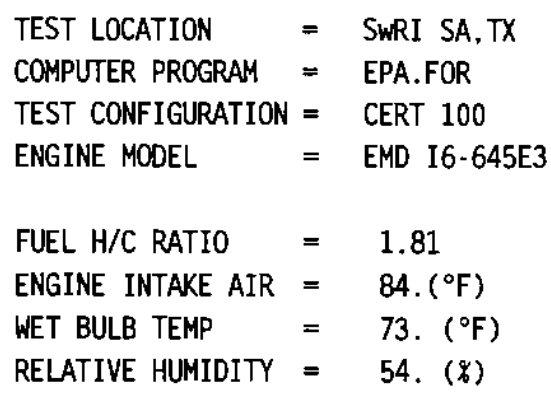

ACCESSORY HORSEPOWER:

$\begin{array}{rlr}\text { AUXILLARY GENERATOR HP } & = & 4.0 \\ \text { AIR COMPRESSOR HP } & = & 6.5 \\ \text { TRACTION MOTOR BLOWER HP } & =6.6 \\ \text { INERTIAL SEPERATOR BLOWER HP } & =1.0 \\ \text { RADIATOR FAN HP } & =3.0 \\ \text { TOTAL ACCESSORY HP } & =21.1\end{array}$

FUEL RATE (OBS) $=132.0(\mathrm{LB} / \mathrm{HR})$

ALT. VOLTS $=377 . \quad$ ALT. AMPS $=$ 553. ALT. EFF. $=.939$

FLYWHEEL HP $=318.9$ OBS. SFC $=.4139$ AAR CORR. SFC $=.4133(\mathrm{LB} / \mathrm{HP} \cdot \mathrm{HR})$

CALCULATED A/F $=93.83$ (LB DRY AIR/LB FUEL)

EMISSIONS G/HR G/HP.HR

$\begin{array}{lrrr}\text { HC } & 164 . & .51 & 60 . \text { PPMC } \\ \text { CO } & 426 . & 1.34 & 79 . \text { PPM } \\ \text { NOX } & 2833 . & 8.88 & 321 . \text { PPM (D) } \\ \text { NOX, CORR } & 3019 . & 9.47 & \\ \text { CO2 } & & 593 . & 2.24 \text { PCT } \\ 02 & & & 17.91 \text { PCT }\end{array}$

CORRECTION FACTOR SUMMARY:

$\begin{array}{lllll}\text { EPA NOX CF }=1.0658 & \text { NOX-KH } & =1.0538 & \text { NOX-KT } & =1.0000 \\ \text { WET EXH HC CF }=.9754 & \text { AIR TEMP CF }=.9892 & \text { BAROM CF } & =1.0031 \\ \text { FUEL TEMP CF }=.9835 & \text { FUEL S.G. CF }=.9907 & \text { FUEL HHV CF } & =1.0062\end{array}$

PARTICULATE INFORMATION

FILTER PAIR I.D. NUMBER

$$
\begin{array}{r}
6775.0 \cdot 60 \\
6.323 \\
37.995 \\
.166 \\
4.295 \\
.715
\end{array}
$$

SAMPLE VOLUME (sCf)

DILUTE PM CONCENTRATION (mg/scf)

PARTICULATE CONCENTRATION IN RAW EXHAUST (mg/scf)

PARTICULATE MASS EMISSION RATE $(\mathrm{g} / \mathrm{hr})$

119.

BRAKE-SPECIFIC PARTICULATE EMISSION RATE $(g / h p-h r)$ 


$\begin{array}{ll}\text { LOCOMOTIVE MODEL } & =\text { EMO GP38 } \\ \text { LOCOMOTIVE UNIT \# } & =\text { CSX } 2629 \\ \text { THROTTLE NOTCH } & =\text { Notch } 3 \\ \text { TEST DATE } & =10 / 30 / 0 \\ \text { TEST NUMBER } & =5 / 10 \\ \text { SWRI FUEL CODE } & =\text { EM-2834-F } \\ \text { BAROMETER } & =29.22(\mathrm{in} . \mathrm{Hg}) \\ \text { DRY BULB TEMP } & =84 .\left({ }^{\circ} \mathrm{F}\right) \\ \text { ABS HUMIDITY } & =103.13(\mathrm{GR} / \mathrm{LB})\end{array}$

$\begin{array}{ll}\text { TEST LOCATION } & =\text { SWRI SA, TX } \\ \text { COMPUTER PROGRAM } & =\text { EPA.FOR } \\ \text { TEST CONFIGURATION } & =\text { CERT } 100 \\ \text { ENGINE MODEL } & =\text { EMD } 16-645 E 3 \\ \text { FUEL H/C RATIO } & =1.81 \\ \text { ENGINE INTAKE AIR } & =80 .\left(^{\circ} \mathrm{F}\right) \\ \text { WET BULB TEMP } & =72 .\left({ }^{\circ} \mathrm{F}\right) \\ \text { RELATIVE HUMIDITY } & =58 .(\%)\end{array}$

ENGINE PERFORMANCE SUMMARY:

ACCESSORY HORSEPOWER:

$\begin{array}{rrr}\text { AUXILLARY GENERATOR HP } & =4.0 \\ \text { AIR COMPRESSOR HP } & =13.3 \\ \text { TRACTION MOTOR BLOWER HP } & =13 \\ \text { INERTIAL SEPERATOR BLOWER HP } & =2.1 \\ \text { RADIATOR FAN HP } & 6.4 \\ \text { TOTAL ACCESSORY HP } & =34.7\end{array}$

FUEL RATE (OBS) $=232.0(\mathrm{LB} / \mathrm{HR})$

ALT. VOLTS $=512 . \quad$ ALT. AMPS $=748 . \quad$ ALT. EFF. $=.939$

FLYWHEEL HP $=581.8 \quad$ OBS. SFC $=.3988$ AAR CORR. SFC $=.3989$ (LB/HP-HR)

CALCULATED A/F $=70.66$ (LB DRY AIR/LB FUEL)

EMISSIONS G/HR G/HP-HR CONC.

$\begin{array}{lrrr}\text { HC } & 254 . & .44 & 70 . \text { PPMC } \\ \text { CO } & 437 . & .75 & 62 . \text { PPM } \\ \text { NOX } & 4379 . & 7.53 & 376 . \text { PPM (D) } \\ \text { NOX,CORR } & 4683 . & 8.05 & \\ \text { CO2 } & & 573 . & 2.99 \text { PCT } \\ 02 & & & 16.78 \text { PCT }\end{array}$

CORRECTION FACTOR SUMMARY:

$\begin{array}{lllll}\text { EPA NOX CF }=1.0696 & \text { NOX-KH } & =1.0569 & \text { NOX-KT } & =1.0000 \\ \text { WET EXH HC CF }=.9689 & \text { AIR TEMP CF }=.9910 & \text { BAROM CF } & =1.0031 \\ \text { FUEL TEMP CF }=.9830 & \text { FUEL S.G. CF }=.9907 & \text { FUEL HHV CF } & =1.0062\end{array}$

PARTICULATE INFORMATION

FILTER PAIR I.D. NUMBER

$6776.0 \cdot 61$

FILTER PAIR WEIGHT GAIN (mg)

5.782

SAMPLE VOLUME (SCf)

38.223

DILUTE PM CONCENTRATION (mg/scf)

.151

5.880

PARTICULATE CONCENTRATION IN RAW EXHAUST (mg/scf)

.889

PARTICULATE MASS EMISSION RATE $(\mathrm{g} / \mathrm{hr})$

197.

8RAKE-SPECIFIC PARTICULATE EMISSION RATE $(\mathrm{g} / \mathrm{hp}-\mathrm{hr})$

.338 


$\begin{array}{ll}\text { LOCOMOTIVE MODEL } & =\text { EMD GP38 } \\ \text { LOCOMOTIVE UNIT \# } & =\text { CSX \#2629 } \\ \text { THROTTLE NOTCH } & =\text { Notch } 4 \\ \text { TEST DATE } & =10 / 30 / 0 \\ \text { TEST NUM8ER } & =6 / 10 \\ \text { SWRI FUEL CODE } & =\text { EM-2834-F } \\ \text { BAROMETER } & =29.21(\mathrm{in} . \mathrm{Hg}) \\ \text { DRY BULB TEMP } & =83 .\left({ }^{\circ} \mathrm{F}\right) \\ \text { ABS HUMIDITY } & =101.66(\mathrm{GR} / \mathrm{LB})\end{array}$

ENGINE PERFORMANCE SUMMARY:

ACCESSORY HORSEPOWER:

$\begin{array}{rlr}\text { AUXILLARY GENERATOR HP } & = & 4.0 \\ \text { AIR COMPRESSOR HP } & = & 9.5 \\ \text { TRACTION MOTOR BLOWER HP } & = & 20.9 \\ \text { INERTIAL SEPERATOR BLOWER HP } & = & 3.1 \\ \text { RADIATOR FAN HP } & =9.7 \\ \text { TOTAL ACCESSORY HP } & =47.3\end{array}$

$\begin{array}{ll}\text { TEST LOCATION } & =\text { SWRI SA,TX } \\ \text { COMPUTER PROGRAM } & =\text { EPA.FOR } \\ \text { TEST CONFIGURATION } & =\text { CERT } 100 \\ \text { ENGINE MODEL } & =\text { EMD } 16.645 E 3 \\ \text { FUEL H/C RATIO } & =1.81 \\ \text { ENGINE INTAKE AIR } & \left.=\text { B2. }{ }^{\circ} \mathrm{F}\right) \\ \text { WET BULB TEMP } & =72 .\left({ }^{\circ} \mathrm{F}\right) \\ \text { RELATIVE HUMIDITY } & =58 .(\%)\end{array}$

392.0 (L8/HR)

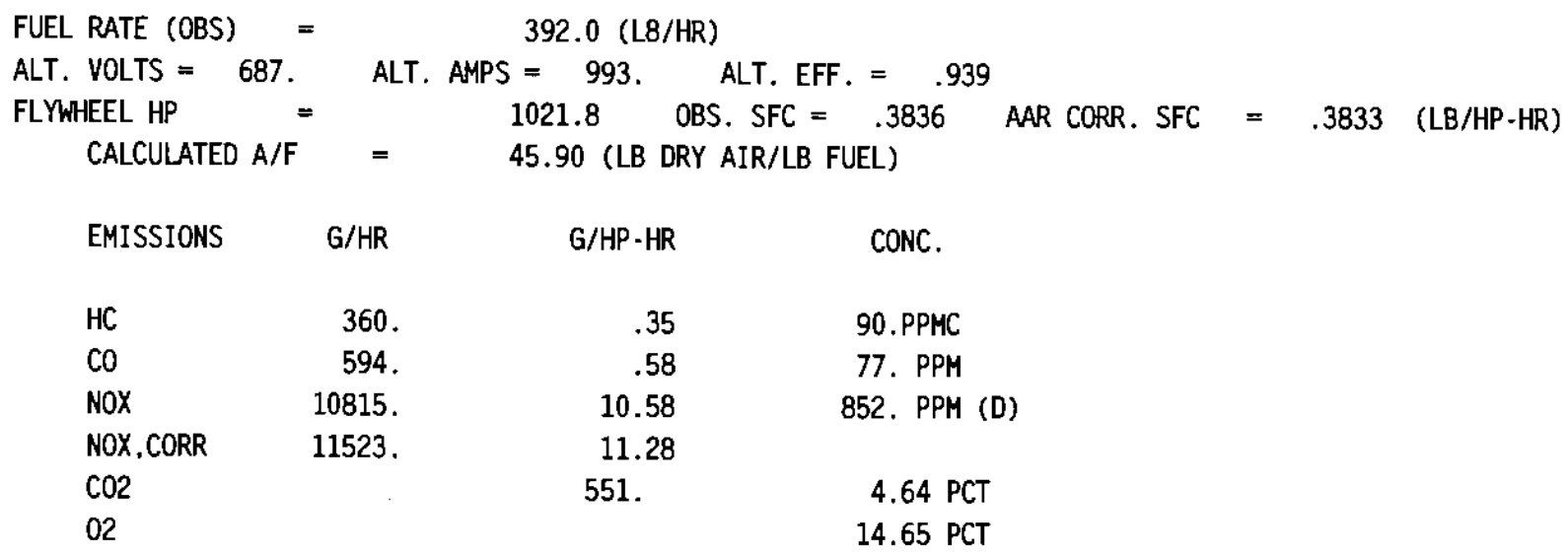

CORRECTION FACTOR SUMMARY:

$\begin{array}{lllll}\text { EPA NOX CF }=1.0655 & \text { NOX-KH } & =1.0535 & \text { NOX-KT } & =1.0000 \\ \text { WET EXH HC CF }=.9551 & \text { AIR TEMP CF }=.9901 & \text { BAROM CF } & =1.0030 \\ \text { FUEL TEMP CF }=.9850 & \text { FUEL S.G. CF }=.9907 & \text { FUEL HHV CF } & =1.0062\end{array}$

PARTICULATE INFORMATION

FILTER PAIR I.D. NUMBER

$6777.0-62$

11.287

37.666

.300

6.003

1.799

440 .

.431 


$\begin{array}{ll}\text { LOCOMOTIVE MODEL } & =\text { EMD GP38 } \\ \text { LOCOMOTIVE UNIT \# } & =\text { CSX } \# 2629 \\ \text { THROTTLE NOTCH } & =\text { Notch } 5 \\ \text { TEST DATE } & =10 / 30 / 0 \\ \text { TEST NUMBER } & =7 / 10 \\ \text { SWRI FUEL CODE } & =\text { EM-2834-F } \\ \text { BAROMETER } & =29.20(\mathrm{in} . \mathrm{Hg}) \\ \text { DRY BULB TEMP } & =84 .\left({ }^{\circ} \mathrm{F}\right) \\ \text { ABS HUMIDITY } & =97.86(\mathrm{GR} / \mathrm{LB})\end{array}$

ENGINE PERFORMANCE SUMMARY:

ACCESSORY HORSEPOWER;

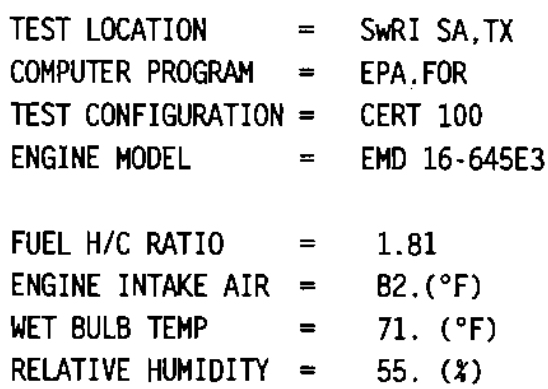

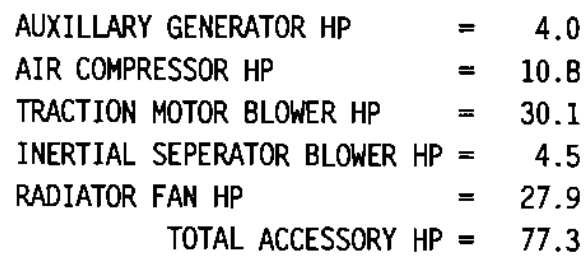

CORRECTION FACTOR SUMMARY:

$\begin{array}{lllll}\text { EPA NOX CF }=1.0559 & \text { NOX-KH } & =1.0457 & \text { NOX-KT } & =1.0000 \\ \text { WET EXH HC CF }=.9509 & \text { AIR TEMP CF }=.9901 & \text { BAROM CF } & =1.0029 \\ \text { FUEL TEMP CF }=.9850 & \text { FUEL S.G. CF }=.9907 & \text { FUEL HHV CF } & =1.0062\end{array}$

PARTICULATE INFORMATION

FILTER PAIR I.D. NUMBER

6778.0.63

8.630

37.265

.232

6.941

1.607

449.

.350 


$\begin{array}{ll}\text { LOCOMOTIVE MODEL } & =\text { EMD GP38 } \\ \text { LOCOMOTIVE UNIT \# } & =\text { CSX } \# 2629 \\ \text { THROTTLE NOTCH } & =\text { Notch } 6 \\ \text { TEST DATE } & =10 / 30 / 0 \\ \text { TEST NUMBER } & =8 / 10 \\ \text { SWRI FUEL CODE } & =\text { EM-2834-F } \\ \text { 8AROMETER } & =29.21(\mathrm{in} . \mathrm{Hg}) \\ \text { DRY 8ULB TEMP } & =83 .\left(^{\circ} \mathrm{F}\right) \\ \text { ABS HUMIDITY } & =97.41(\mathrm{GR} / \mathrm{LB})\end{array}$

ENGINE PERFORMANCE SUMMARY:

ACCESSORY HORSEPOWER:

$\begin{array}{rlr}\text { AUXILLARY GENERATOR HP } & =4.0 \\ \text { AIR COMPRESSOR HP } & =12.2 \\ \text { TRACTION MOTOR BLOWER HP } & 43.5 \\ \text { INERTIAL SEPERATOR BLOWER HP } & =6.5 \\ \text { RADIATOR FAN HP } & 40.2 \\ \text { TOTAL ACCESSORY HP } & =106.4\end{array}$

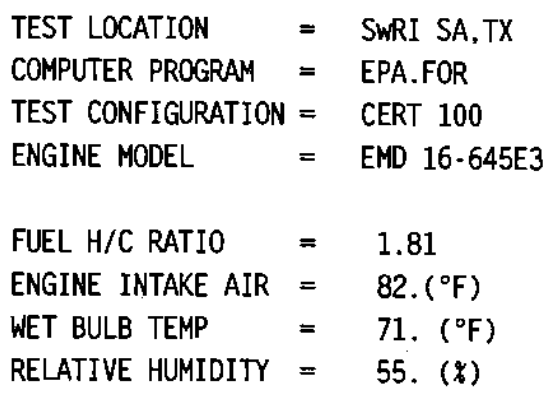

FUEL RATE (OBS) = $618.0(\mathrm{LB} / \mathrm{HR})$

ALT. VOLTS $=844 . \quad$ ALT. AMPS $=1206 . \quad$ ALT. EFF. $=.939$

FLYWHEEL HP $=1560.5 \quad$ OBS. SFC $=.3960 \quad$ AAR CORR. SFC $=\quad .3957$ (LB/HP-HR)

CALCULATED A/F $=37.29$ (LB DRY AIR/LB FUEL)

EMISSIONS G/HR G/HP-HR CONC.

$\begin{array}{llll}\text { HC } & 644 . & .41 & 125 . \text { PPMC }\end{array}$

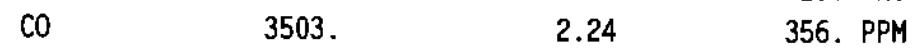

NOX 18320. $11.74 \quad 1132$. PPM (D)

NOX,CORR $19322 . \quad 12.38$

$02 \quad 13.15$ PCT

CORRECTION FACTOR SUMMARY:

$\begin{array}{lllll}\text { EPA NOX CF }=1.0547 & \text { NOX-KH } & =1.0448 & \text { NOX-KT } & =1.0000 \\ \text { WET EXH HC CF }=.9463 & \text { AIR TEMP CF }=.9901 & \text { BAROM CF } & =1.0030 \\ \text { FUEL TEMP CF }=.9850 & \text { FUEL S.G. CF }=.9907 & \text { FUEL HHV CF } & =1.0062\end{array}$

PARTICULATE INFORMATION

FILTER PAIR I.D. NUMBER

$6779.0-64$

FILTER PAIR WEIGHT GAIN (mg)

8.328

SAMPLE VOLUME (sCf)

37.073

DILUTE PM CONCENTRATION (mg/scf)

CO2-BASED DILUTION FACTOR $\quad 7.963$

PARTICULATE CONCENTRATION IN RAW EXHAUST (mg/scf) 1.789

PARTICULATE MASS EMISSION RATE $(\mathrm{g} / \mathrm{hr})$

BRAKE-SPECIFIC PARTICULATE EMISSION RATE $(\mathrm{g} / \mathrm{hp}-\mathrm{hr}) \quad .361$ 


$\begin{array}{ll}\text { LOCOMOTIVE MODEL } & =\text { EMD GP38 } \\ \text { LOCOMOTIVE UNIT \# } & =\text { CSX } \# 2629 \\ \text { TROTTLE NOTCH } & =\text { Notch } 7 \\ \text { TEST DATE } & =10 / 30 / 0 \\ \text { TEST NUMBER } & =9 / 10 \\ \text { SWRI FUEL CODE } & =\text { EM-2834-F } \\ \text { BAROMETER } & =29.20(\mathrm{in} . \mathrm{Hg}) \\ \text { DRY BULB TEMP } & =83 .\left({ }^{\circ} \mathrm{F}\right) \\ \text { ABS HUMIDITY } & =100.29(\mathrm{GR} / \mathrm{LB})\end{array}$

\section{ENGINE PERFORMANCE SUMMARY:}

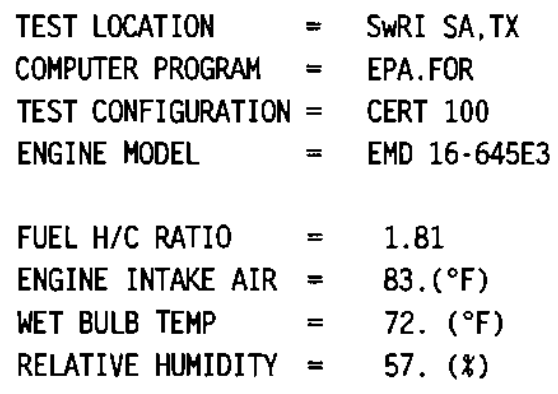

ACCESSORY HORSEPOWER:

$\begin{array}{rlr}\text { AUXILLARY GENERATOR HP } & = & 4.0 \\ \text { AIR COMPRESSOR HP } & = & 13.8 \\ \text { TRACTION MOTOR BLOWER HP } & =62.8 \\ \text { INERTIAL SEPERATOR BLOWER HP } & =9.4 \\ \text { RADIATOR FAN HP } & =58.1 \\ \text { TOTAL ACCESSORY HP } & =148.0\end{array}$

FUEL RATE (OBS) $=766.0$ (LB/HR)

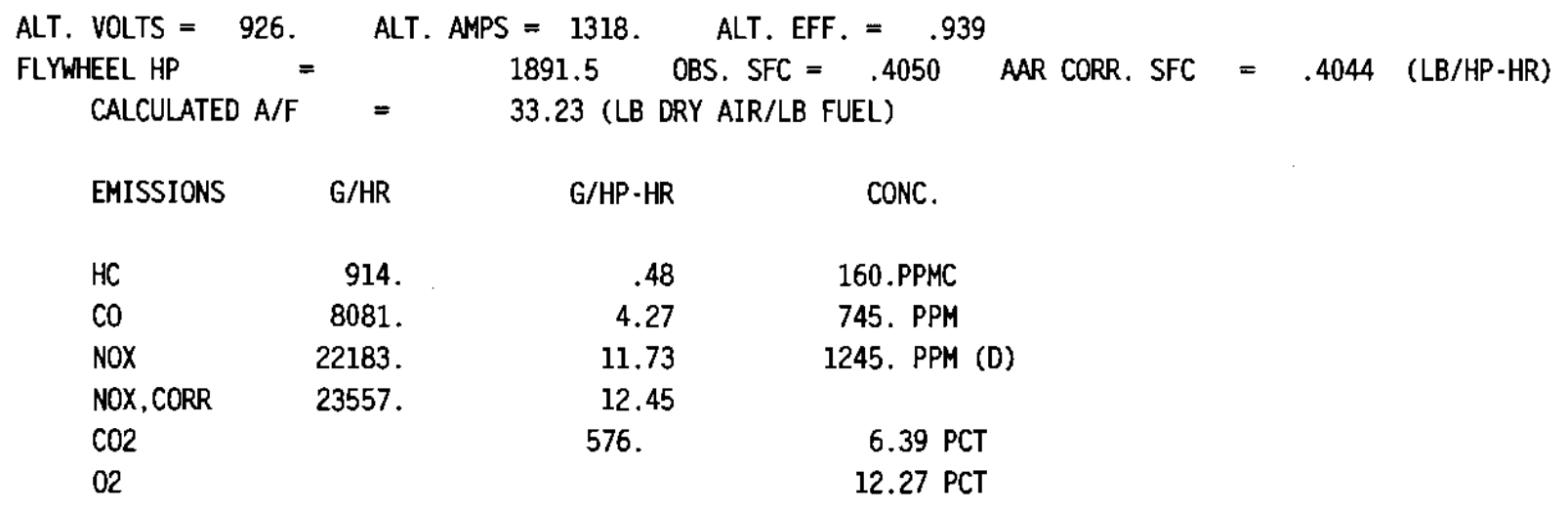

CORRECTION FACTOR SUMMARY:

\begin{tabular}{|c|c|c|c|c|c|}
\hline & $=1.0619$ & $\mathrm{NOX} \cdot \mathrm{KH}$ & $=1.0507$ & NOX-KT & $=1.0000$ \\
\hline $\mathrm{HC}$ & .9405 & AIR TEMP CF & $=.9896$ & BAROM CF & $=1.0029$ \\
\hline UEL & .9845 & FUEL S.G. CF & .9907 & FUEL HHV CF & 1.0062 \\
\hline
\end{tabular}

PARTICULATE INFORMATION

FILTER PAIR I.D. NUMBER

$6780.0-65$

FILTER PAIR WEIGHT GAIN (mg)

12.253

36.537

SAMPLE VOLUME (sCf)

.335

DILUTE PM CONCENTRATION (mg/scf)

7.881

CO2-BASED DILUTION FACTOR

2.643

PARTICULATE CONCENTRATION IN RAW EXHAUST ( $\mathrm{mg} / \mathrm{scf}$ )

PARTICULATE MASS EMISSION RATE $(\mathrm{g} / \mathrm{hr})$

922.

BRAKE-SPECIFIC PARTICULATE EMISSION RATE $(g / h p-h r)$ 


$\begin{array}{ll}\text { LOCOMOTIVE MODEL } & =\text { EMD GP38 } \\ \text { LOCOMOTIVE UNIT \# } & =\text { CSX } \# 2629 \\ \text { THROTTLE NOTCH } & =\text { Notch } 8 \\ \text { TEST DATE } & =10 / 30 / 0 \\ \text { TEST NUMBER } & =10 / 10 \\ \text { SWRI FUEL CODE } & =\text { EM }-2834-\mathrm{F} \\ \text { BAROMETER } & =29.19(\mathrm{in} . \mathrm{Hg}) \\ \text { DRY BULB TEMP } & =83 .\left(^{\circ} \mathrm{F}\right) \\ \text { ABS HUMIDITY } & =98.00(\mathrm{GR} / \mathrm{LB})\end{array}$

ENGINE PERFORMANCE SUMMARY:

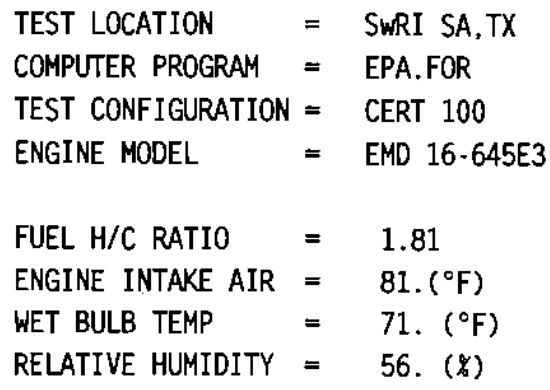

ACCESSORY HORSEPOWER:

$\begin{array}{rrr}\text { AUXILLARY GENERATOR HP } & =4.0 \\ \text { AIR COMPRESSOR HP } & =15.1 \\ \text { TRACTION MOTOR BLOWER HP } & \mathrm{B} 2.8 \\ \text { INERTIAL SEPERATOR BLOWER HP } & =12.4 \\ \text { RADIATOR FAN HP } & 76.6 \\ \text { TOTAL ACCESSORY HP } & =191.0\end{array}$

FUEL RATE (OBS) = 908.0 (LB/HR)

ALT. VOLTS $=980 . \quad$ ALT. AMPS $=1388 . \quad$ ALT. EFF. $=.939$

FLYWHEEL HP $=\quad 2134.2$ OBS. SFC $=.4255$ AAR CORR. SFC $=.4253$ (LB/HP-HR)

CALCULATED A/F $=30.85$ (LB DRY AIR/LB FUEL)

EMISSIONS G/HR G/HP-HR CONC.

$\begin{array}{lrrr}\text { HC } & 1228 . & .58 & 195 . \text { PPMC } \\ \text { CO } & 15743 . & 7.38 & 1321 . \text { PPM } \\ \text { NOX } & 24127 . & 11.31 & 1232 . \text { PPM (D) } \\ \text { NOX,CORR } & 25483 . & 11.94 & \\ \text { C02 } & & 600 . & 6.84 \text { PCT } \\ 02 & & & 11.52 \text { PCT }\end{array}$

CORRECTION FACTOR SUMMARY:

$\begin{array}{lllll}\text { EPA NOX CF }=1.0562 & \text { NOX-KH } & =1.0460 & \text { NOX-KT } & =1.0000 \\ \text { WET EXH HC CF }=.9367 & \text { AIR TEMP CF }=.9905 & \text { BAROM CF } & =1.0029 \\ \text { FUEL TEMP CF }=.9860 & \text { FUEL S.G. CF }=.9907 & \text { FUEL HHV CF } & =1.0062\end{array}$

PARTICULATE INFORMATION

FILTER PAIR I.D. NUMBER

FILTER PAIR WEIGHT GAIN (mg)

SAMPLE VOLUME (SCf)

DILUTE PM CONCENTRATION (mg/scf)

CO2-BASED DILUTION FACTOR

PARTICULATE CONCENTRATION IN RAW EXHAUST ( $\mathrm{mg} / \mathrm{scf}$ )

PARTICULATE MASS EMISSION RATE $(\mathrm{g} / \mathrm{hr})$

BRAKE-SPECIFIC PARTICULATE EMISSION RATE $(\mathrm{g} / \mathrm{hp}-\mathrm{hr})$

\author{
$6781.0-66$ \\ 11.577 \\ 35.551 \\ .326 \\ 9.150 \\ 2.980 \\ 1147.
}

.537 
|.

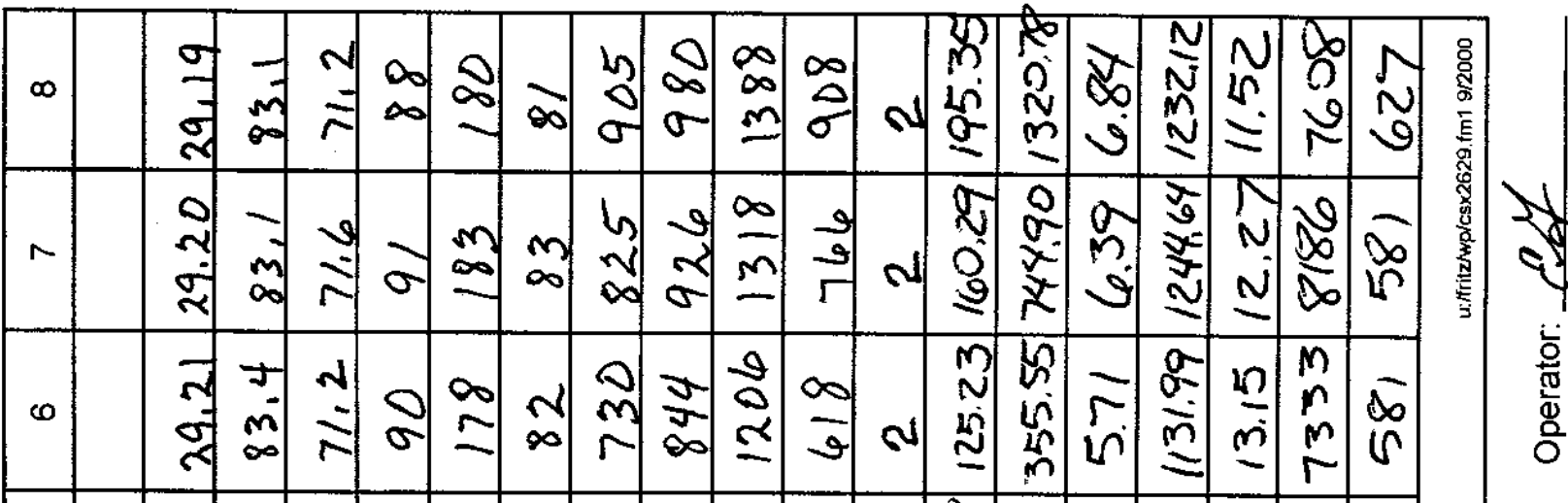

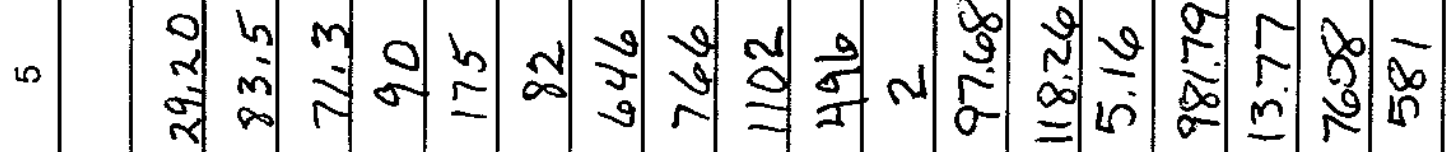

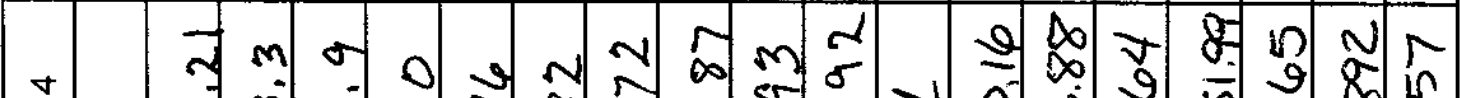

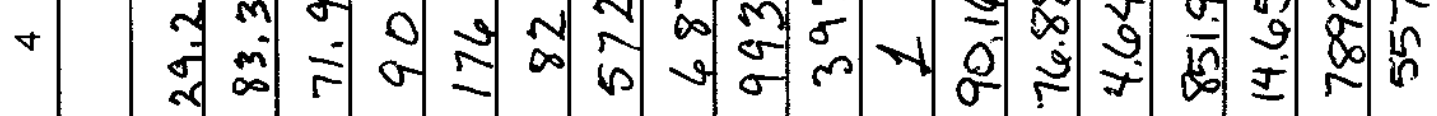

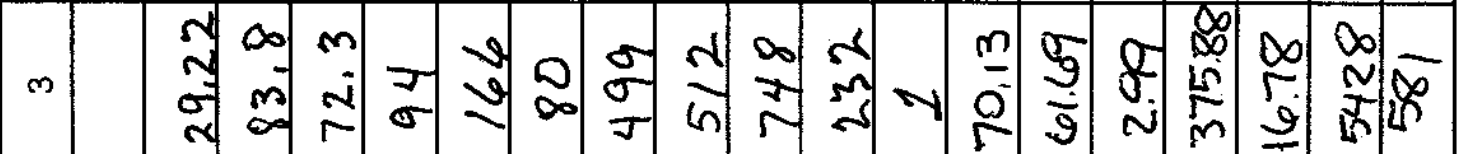

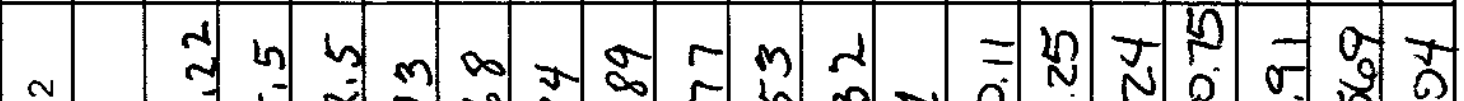

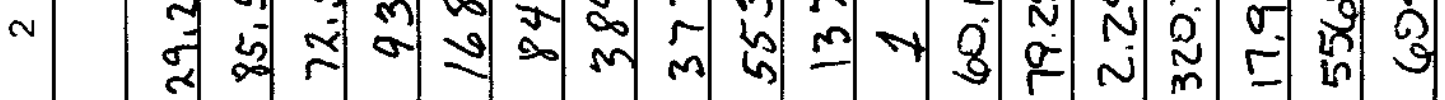

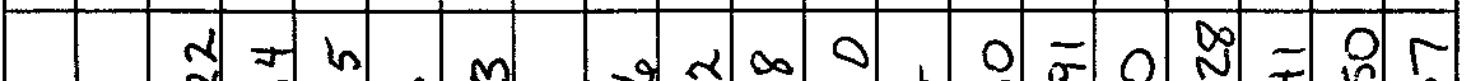
- ने चे

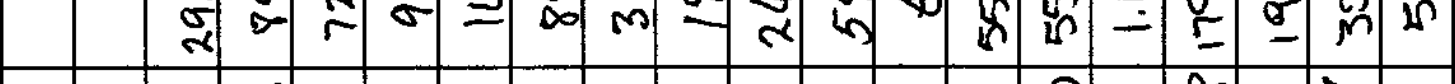

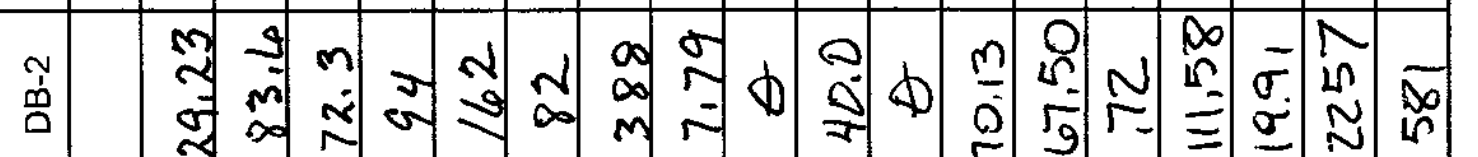

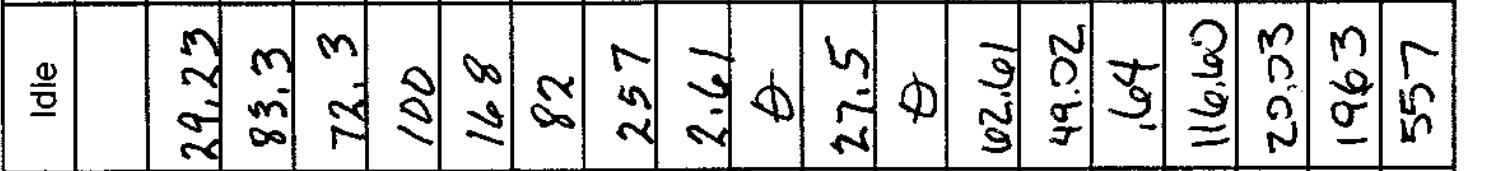

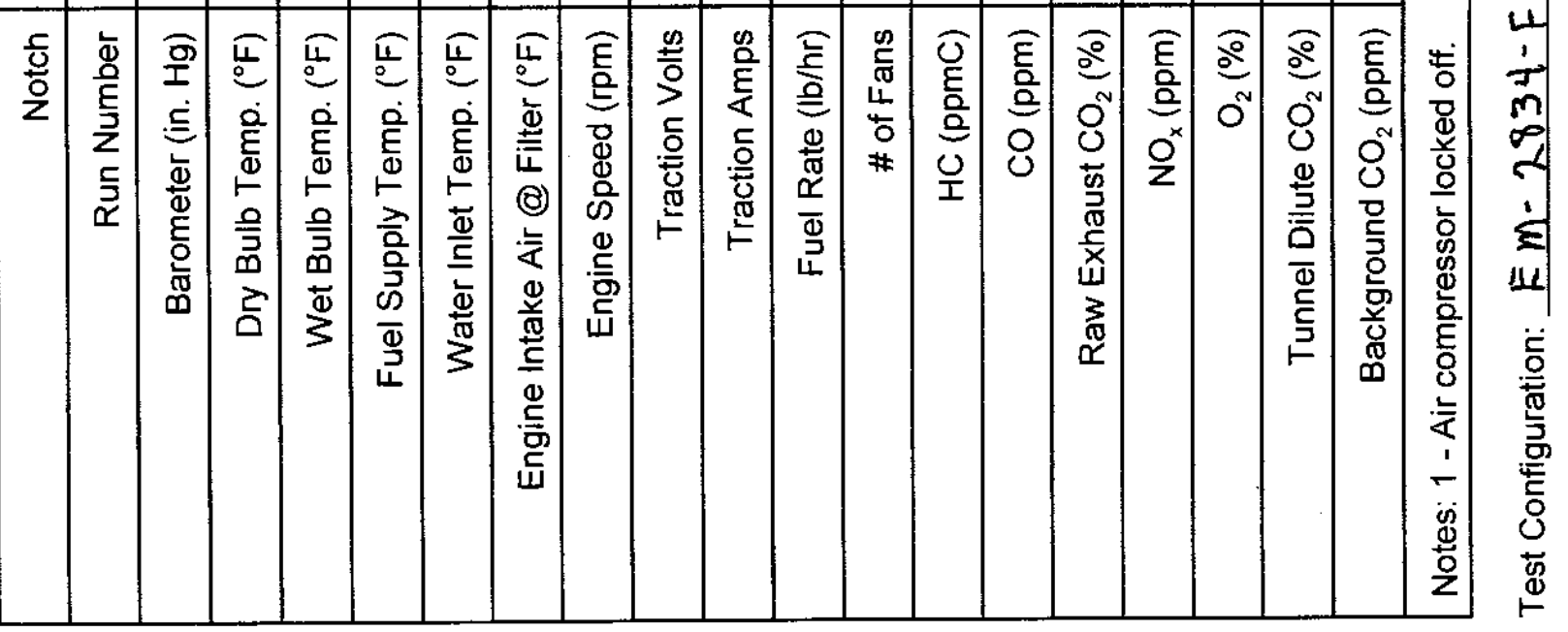




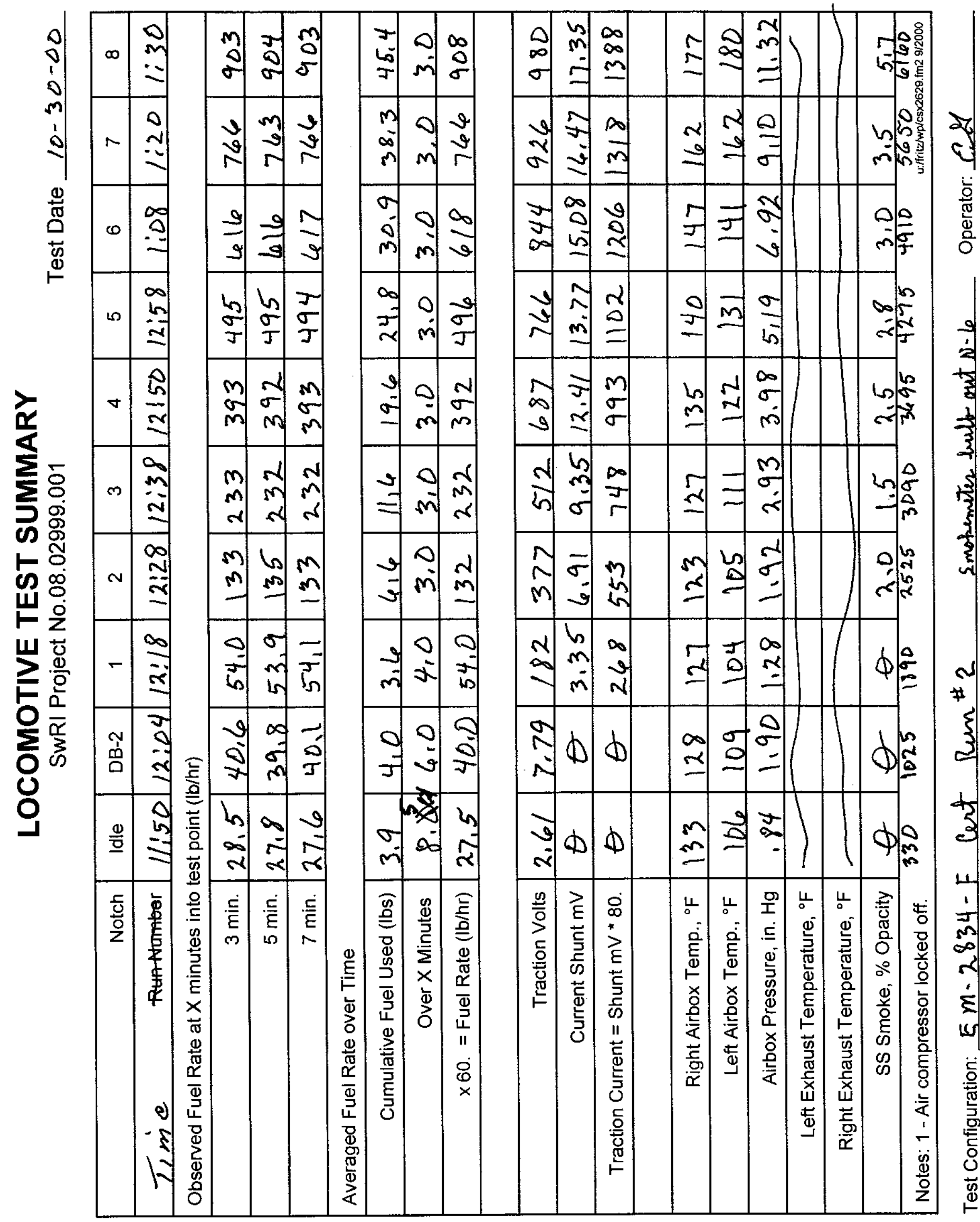




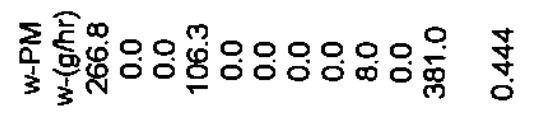

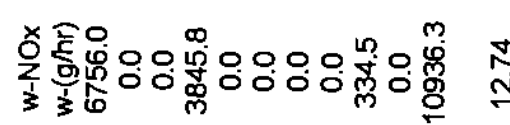

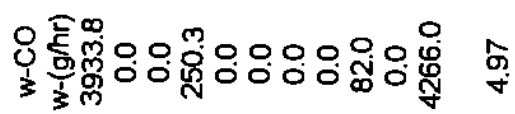

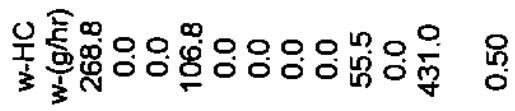

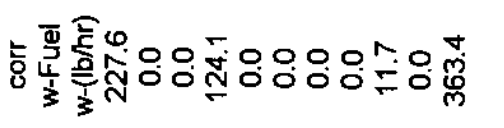

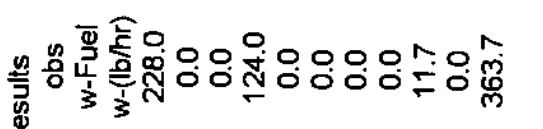

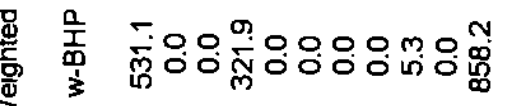

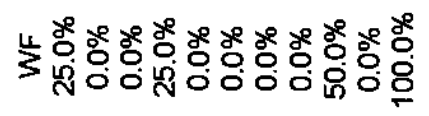
言产产

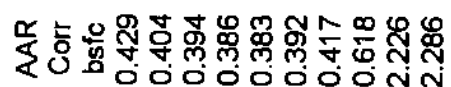

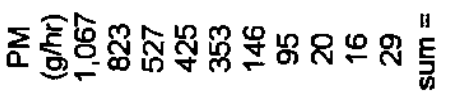

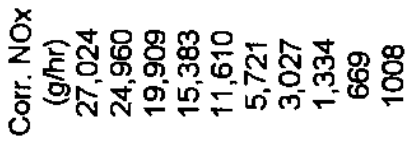

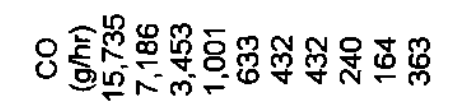

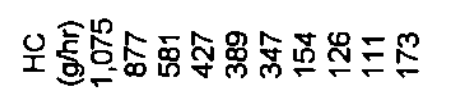

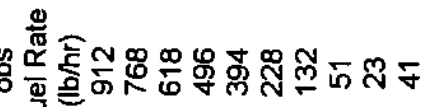

일

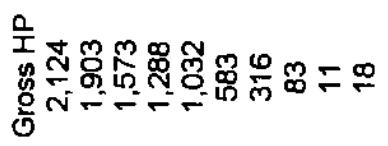

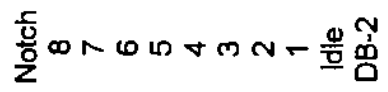

突产

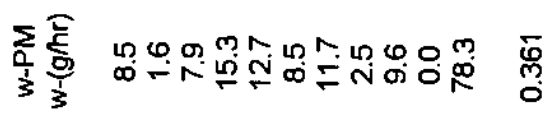

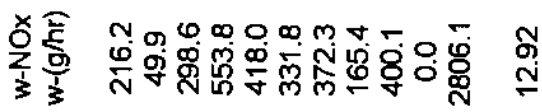

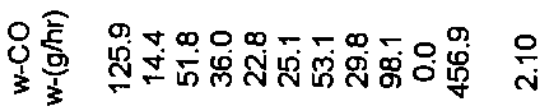

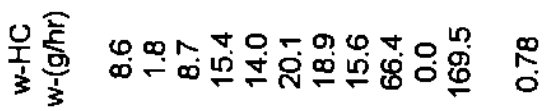

兽

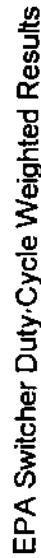

产

崖

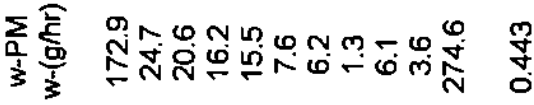

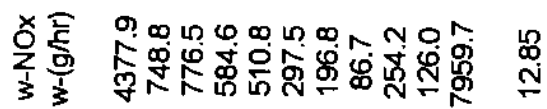

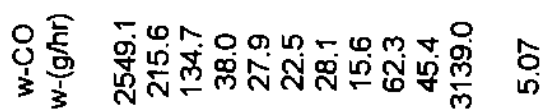

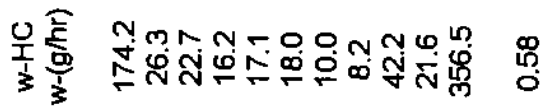

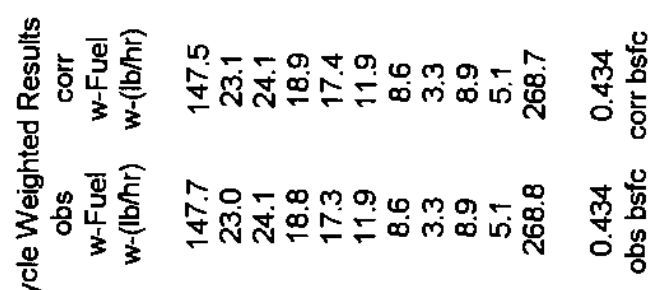

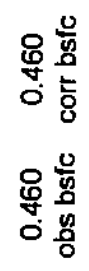

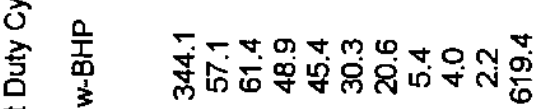

แ พ้ำ

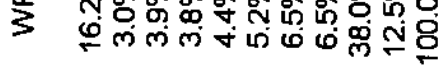

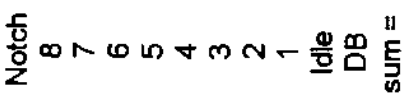

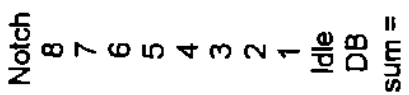




$\begin{array}{ll}\text { LOCOMOTIVE MODEL } & =\text { EMD GP38 } \\ \text { LOCOMOTIVE UNIT \# } & =\text { CSX } \# 2629 \\ \text { THROTTLE NOTCH } & =\text { IDLE } \\ \text { TEST DATE } & =11 / 1 / 0 \\ \text { TEST NUMBER } & =1 / 10 \\ \text { SWRI FUEL CODE } & =\text { EM }-2834-\mathrm{F} \\ \text { BAROMETER } & =29.09(\mathrm{in} . \mathrm{Hg}) \\ \text { DRY BULB TEMP } & =77 .\left({ }^{\circ} \mathrm{F}\right) \\ \text { ABS HUMIDITY } & =115.48(\mathrm{GR} / \mathrm{LB})\end{array}$

ENGINE PERFORMANCE SUMMARY:

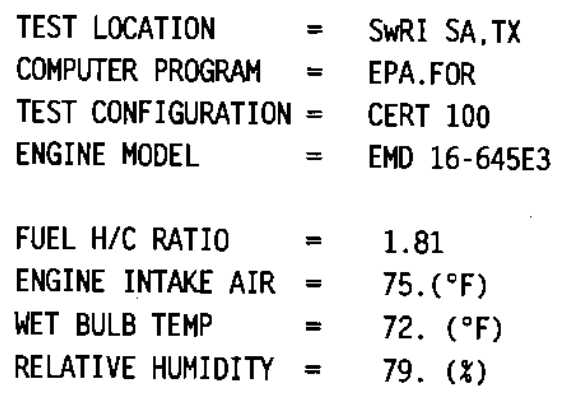

\section{ACCESSORY HORSEPOWER:}

$\begin{array}{rrr}\text { AUXILLARY GENERATOR HP } & = & 4.0 \\ \text { AIR COMPRESSOR HP } & = & 4.3 \\ \text { TRACTION MOTOR BLOWER HP } & =1.9 \\ \text { INERTIAL SEPERATOR BLOWER HP } & =.3 \\ \text { RADIATOR FAN HP } & =.0 \\ \text { TOTAL ACCESSORY HP } & =10.5\end{array}$

FUEL RATE (OBS) = $23.3(\mathrm{LB} / \mathrm{HR})$

ALT. VOLTS $=3 . \quad$ ALT. AMPS $=0 . \quad$ ALT. EFF. $=.939$

FLYWHEEL HP $=10.5$ OBS. SFC $=2.2231 \quad$ AAR CORR. SFC $=2.2263$ (LB/HP-HR)

CALCULATED A/F $=361.62$ (LB DRY AIR/LB FUEL)

EMISSIONS G/HR G/HP-HR CONC.

$\begin{array}{llll}H C & 111 & 10.55 & 60 . \text { PPMC }\end{array}$

$\begin{array}{llll}\mathrm{CO} & 164 . & 15.63 & 44 . \mathrm{PPM}\end{array}$

NOX 599. $57.20 \quad$ 99. PPM (D)

$\begin{array}{lll}\text { NOX, CORR } & 669 . & 63.80\end{array}$

$\begin{array}{lll}\mathrm{CO} 2 & 3149 . & .57 \text { PCT }\end{array}$

$02 \quad 20.03$ PCT

CORRECTION FACTOR SUMMARY:

$\begin{array}{lllll}\text { EPA NOX CF }=1.1154 & \text { NOX-KH } & =1.0940 & \text { NOX-KT } & =1.0000 \\ \text { WET EXH HC CF }=.9894 & \text { AIR TEMP CF }=.9932 & \text { BAROM CF } & =1.0020 \\ \text { FUEL TEMP CF }=.9835 & \text { FUEL S.G. CF }=.9907 & \text { FUEL HHV CF } & =1.0062\end{array}$

PARTICULATE INFORMATION

FILTER PAIR I.D. NUMBER

FILTER PAIR WEIGHT GAIN (mg)

$6822.0-107$

1.234

SAMPLE VOLUME (SCf)

39.075

DILUTE PM CONCENTRATION (mg/scf)

.032

CO2-BASED DILUTION FACTOR

4.578

PARTICULATE CONCENTRATION IN RAW EXHAUST (mg/scf)

PARTICULATE MASS EMISSION RATE $(\mathrm{g} / \mathrm{hr})$

.145

BRAKE-SPECIFIC PARTICULATE EMISSION RATE $(g / h p-h r)$

16.

1.551 


$\begin{array}{ll}\text { LOCOMOTIVE MODEL } & =\text { EMD GP38 } \\ \text { LOCOMOTIVE UNIT \# } & =\text { CSX \#2629 } \\ \text { THROTTLE NOTCH } & =\text { DB2 } \\ \text { TEST DATE } & =11 / 1 / 0 \\ \text { TEST NUMBER } & =2 / 10 \\ \text { SWRI FUEL CODE } & =\text { EM-2834-F } \\ \text { BAROMETER } & =29.09(\mathrm{in} . \mathrm{Hg}) \\ \text { DRY BULB TEMP } & =78 .\left({ }^{\circ} \mathrm{F}\right) \\ \text { ABS HUMIDITY } & =115.82(\mathrm{GR} / \mathrm{LB})\end{array}$

$\begin{array}{ll}\text { TEST LOCATION } & \text { SWRI SA,TX } \\ \text { COMPUTER PROGRAM } & =\text { EPA.FOR } \\ \text { TEST CONFIGURATION } & =\text { CERT } 100 \\ \text { ENGINE MODEL } & =\text { EMD } 16 \cdot 645 E 3 \\ \text { FUEL H/C RATIO } & =1.81 \\ \text { ENGINE INTAKE AIR } & =77 .\left({ }^{\circ} \mathrm{F}\right) \\ \text { WET BULB TEMP } & =73 .\left({ }^{\circ} \mathrm{F}\right) \\ \text { RELATIVE HUMIDITY } & =78 .\left({ }^{\circ}\right)\end{array}$

ENGINE PERFORMANCE SUMMARY:

ACCESSORY HORSEPOWER:

$\begin{array}{rlr}\text { AUXILLARY GENERATOR HP } & = & 4.0 \\ \text { AIR COMPRESSOR HP } & = & 6.4 \\ \text { TRACTION MOTOR BLOWER HP } & =6.5 \\ \text { INERTIAL SEPERATOR BLOWER HP } & =1.0 \\ \text { RADIATOR FAN HP } & .0 \\ \text { TOTAL ACCESSORY HP } & =17.9\end{array}$

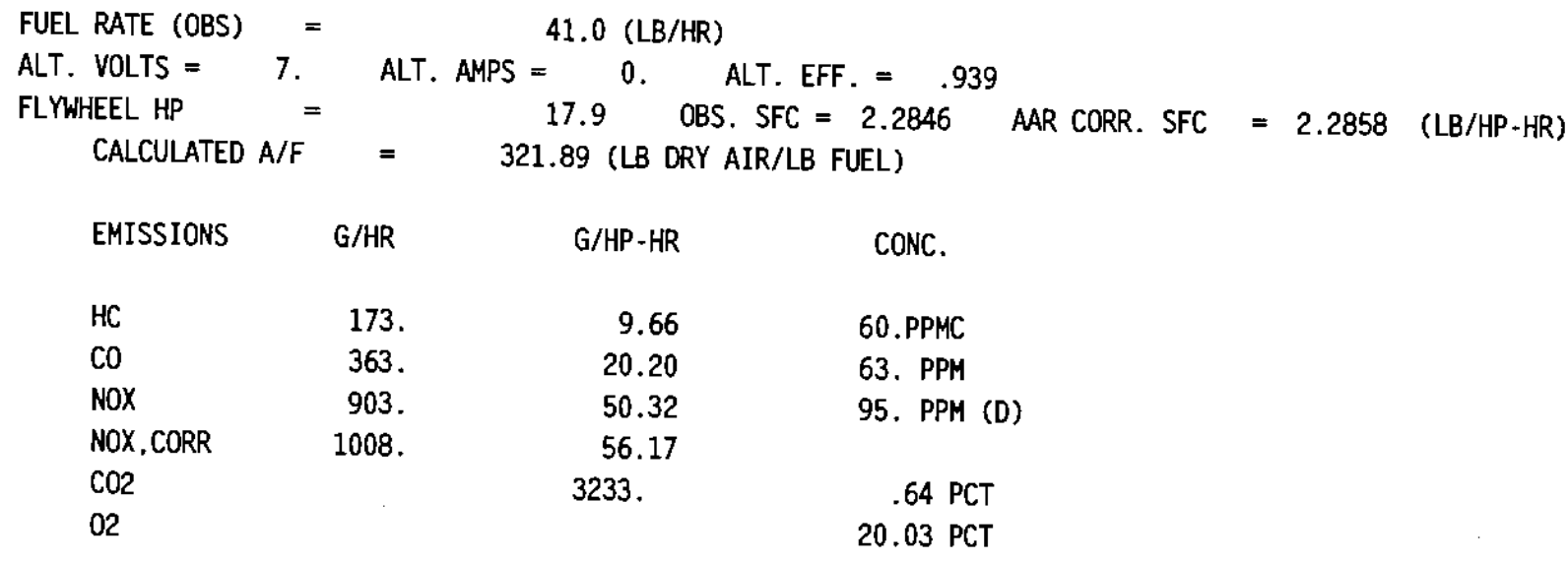

CORRECTION FACTOR SUMMARY:

$\begin{array}{lllll}\text { EPA NOX CF }=1.1163 & \text { NOX-KH } & =1.0947 & \text { NOX-KT } & =1.0000 \\ \text { WET EXH HC CF }=.9887 & \text { AIR TEMP CF }=.9923 & \text { BAROM CF } & =1.0020 \\ \text { FUEL TEMP CF }=.9825 & \text { FUEL S.G. CF }=.9907 & \text { FUEL HHV CF } & =1.0062\end{array}$

PARTICULATE INFORMATION

FILTER PAIR I.D. NUMBER

$6823.0-108$
1.607
38.668
.042
3.912
.163
29.
1.596

FILTER PAIR WEIGHT GAIN (mg)

SAMPLE VOLUME (SCF)

DILUTE PM CONCENTRATION (mg/scf)

CO2-BASED DILUTION FACTOR

PARTICULATE CONCENTRATION IN RAW EXHAUST (mg/scf)

PARTICULATE MASS EMISSION RATE $(\mathrm{g} / \mathrm{hr})$

BRAKE-SPECIFIC PARTICULATE EMISSION RATE $(g / h p \cdot h r)$

1.596 


$\begin{array}{ll}\text { LOCOMOTIVE MODEL } & =\text { EMD GP38 } \\ \text { LOCOMOTIVE UNIT \# } & =\text { CSX } \# 2629 \\ \text { THROTTLE NOTCH } & =\text { Notch } 1 \\ \text { TEST DATE } & =11 / 1 / 0 \\ \text { TEST NUMBER } & =3 / 10 \\ \text { SWRI FUEL CODE } & =\text { EM-2834-F } \\ \text { BAROMETER } & =29.09(\mathrm{in} . \mathrm{Hg}) \\ \text { DRY 8ULB TEMP } & =78 .\left(^{\circ} \mathrm{F}\right) \\ \text { ABS HUMIDITY } & =118.16(\mathrm{GR} / \mathrm{LB})\end{array}$

ENGINE PERFORMANCE SUMMARY:

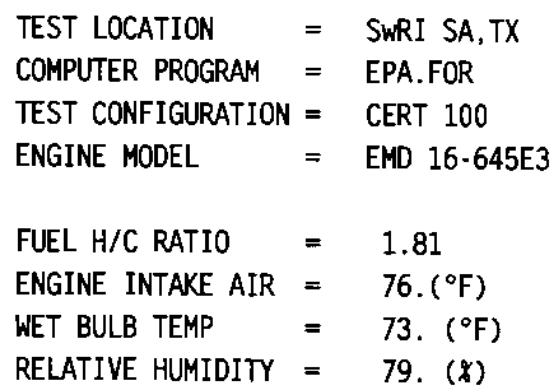

ACCESSORY HORSEPOWER:

$\begin{array}{rlr}\text { AUXILLARY GENERATOR HP } & = & 4.0 \\ \text { AIR COMPRESSOR HP } & = & 5.3 \\ \text { TRACTION MOTOR BLOWER HP } & = & 3.5 \\ \text { INERTIAL SEPERATOR BLOWER HP } & = & .5 \\ \text { RADIATOR FAN HP } & =0 \\ \text { TOTAL ACCESSORY HP } & =13.3\end{array}$

FUEL RATE (08S) =

51.0 (LB/HR)

ALT. VOLTS $=181$.

ALT. AMPS $=268 . \quad$ ALT. EFF, $=.939$

FLYWHEEL HP =

82.6 OBS. $\mathrm{SFC}=.6175$

CALCULATED A/F $=214.51$ (LB DRY AIR/LB FUEL)

EMISSIONS G/HR G/HP.HR CONC.

$\begin{array}{lrrr}\text { HC } & 126 . & 1.52 & 53 . P P M C \\ \text { CO } & 240 . & 2.90 & 50 . \text { PPM } \\ \text { NOX } & 1190 . & 14.41 & 152 . \text { PPM (D) } \\ \text { NOX, CORR } & 1334 . & 16.15 & \\ \text { CO2 } & & 881 . & .97 \text { PCT } \\ 02 & & 19.53 \text { PCT }\end{array}$

CORRECTION FACTOR SUMMARY:

$\begin{array}{lllll}\text { EPA NOX CF }=1.1205 & \text { NOX-KH } & =1.0982 & \text { NOX-KT } & =1.0000 \\ \text { WET EXH HC CF }=.9857 & \text { AIR TEMP CF }=.9928 & \text { BAROM CF } & =1.0020 \\ \text { FUEL TEMP CF }=.9840 & \text { FUEL S.G. CF }=.9907 & \text { FUEL HHV CF } & =1.0062\end{array}$

PARTICULATE INFORMATION

FILTER PAIR I.D. NUMBER

$6824.0-109$

FILTER PAIR WEIGHT GAIN (mg)

1.518

SAMPLE VOLUME (SCf)

38.248

DILUTE PM CONCENTRATION (mg/scf)

.040

CO2-BASED DILUTION FACTOR

3.497

PARTICULATE CONCENTRATION IN RAW EXHAUST (mg/scf)

PARTICULATE MASS EMISSION RATE $(\mathrm{g} / \mathrm{hr})$

.139

20.

BRAKE-SPECIFIC PARTICULATE EMISSION RATE $(\mathrm{g} / \mathrm{hp}-\mathrm{hr})$ 


$\begin{array}{ll}\text { LOCOMOTIVE MODEL } & =\text { EMD GP38 } \\ \text { LOCOMOTIVE UNIT \# } & =\text { CSX } \# 2629 \\ \text { THROTTLE NOTCH } & =\text { Notch } 2 \\ \text { TEST DATE } & =11 / 1 / 0 \\ \text { TEST NUMBER } & =4 / 10 \\ \text { SWRI FUEL CODE } & =E M-2834-\mathrm{F} \\ \text { BAROMETER } & =29.08(\mathrm{in} . \mathrm{Hg}) \\ \text { DRY BULB TEMP } & \left.=78 .{ }^{\circ} \mathrm{F}\right) \\ \text { ABS HUMIDITY } & =113.88(\mathrm{GR} / \mathrm{LB})\end{array}$

ENGINE PERFORMANCE SUMMARY:

ACCESSORY HORSEPOWER:

$\begin{array}{rlr}\text { AUXILLARY GENERATOR HP } & =4.0 \\ \text { AIR COMPRESSOR HP } & =6.5 \\ \text { TRACTION MOTOR BLOWER HP } & =6.6 \\ \text { INERTIAL SEPERATOR BLOWER HP } & =1.0 \\ \text { RADIATOR FAN HP } & =3.1 \\ \text { TOTAL ACCESSORY HP } & =21.2\end{array}$

FUEL RATE (OBS) =

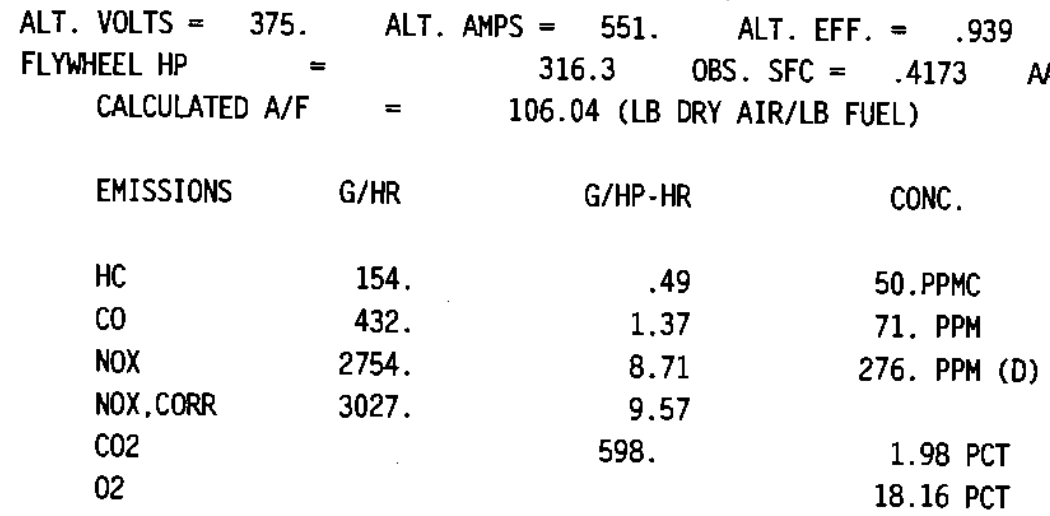

CORRECTION FACTOR SUMMARY:

$\begin{array}{lllll}\text { EPA NOX CF }=1.0990 & \text { NOX-KH } & =1.0808 & \text { NOX-KT } & =1.0000 \\ \text { WET EXH HC CF }=.9770 & \text { AIR TEMP CF }=.9919 & \text { BAROM CF } & =1.0019 \\ \text { FUEL TEMP CF }=.9830 & \text { FUEL S.G. CF }=.9907 & \text { FUEL HHV CF } & =1.0062\end{array}$

PARTICULATE INFORMATION

FILTER PAIR I.D. NUMBER

FILTER PAIR WEIGHT GAIN (mg)

$6825.0 \cdot 110$

SAMPLE VOLUME (scf)

4.165

38.395

DILUTE PM CONCENTRATION (mg/scf)

.108

4.638

PARTICULATE CONCENTRATION IN RAW EXHAUST (mg/scf)

PARTICULATE MASS EMISSION RATE $(\mathrm{g} / \mathrm{hr})$

.503

95.

BRAKE-SPECIFIC PARTICULATE EMISSION RATE $(\mathrm{g} / \mathrm{hp} \cdot \mathrm{hr})$

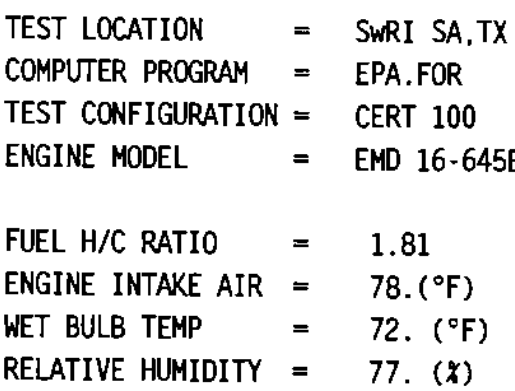


LOCOMOTIVE EMISSIONS SWRI PROJECT NO. 08-2999-001

\begin{tabular}{|c|c|c|c|}
\hline LOCOMOTIVE MODEL & EMD GP38 & TEST LOCATION & SWRI SA,TX \\
\hline LOCOMOTIVE UNIT \# & $\operatorname{cS} x \# 2629$ & COMPUTER PROGRAM & EPA.FOR \\
\hline THROTTLE NOTCH & Notch 3 & TEST CONFIGURATION & CERT 100 \\
\hline TEST DATE & $11 / 1 / 0$ & ENGINE MODEL & EMD $16-645 E 3$ \\
\hline TEST NUMBER & $5 / 10$ & & \\
\hline SWRI FUEL CODE & $E M-2 B 34-F$ & FUEL H/C RATIO & $1 . \mathrm{B} 1$ \\
\hline BAROMETER & $29.09(\mathrm{in.} H \mathrm{Hg})$ & ENGINE INTAKE AIR & $75 .\left(^{\circ} \mathrm{F}\right)$ \\
\hline DRY BULB TEMP & $=77 .\left({ }^{\circ} \mathrm{F}\right)$ & WET BULB TEMP & 72. $\left({ }^{\circ} \mathrm{F}\right)$ \\
\hline ABS HUMIDITY & $=112.07($ GR/LB $)$ & RELATIVE HUMIDITY & 77. (y) \\
\hline
\end{tabular}

ENGINE PERFORMANCE SUMMARY:

ACCESSORY HORSEPOWER:

$\begin{array}{rlr}\text { AUXILLARY GENERATOR HP } & = & 4.0 \\ \text { AIR COMPRESSOR HP } & = & 8.3 \\ \text { TRACTION MOTOR BLOWER HP } & = & 14.1 \\ \text { INERTIAL SEPERATOR BLOWER HP } & = & 2.1 \\ \text { RADIATOR FAN HP } & = & 6.5 \\ \text { TOTAL ACCESSORY HP } & = & 35.0\end{array}$

FUEL RATE (OBS) $=228.0$ (LB/HR)

ALT. VOLTS $=$ 513. ALT. AMPS $=748 . \quad$ ALT. EFF. $=.939$

FLYWHEEL HP $=583.2$ OBS. SFC $=.3909$ AAR CORR. SFC $=.3915$ (LB/HP-HR)

CALCULATED A/F $=76.67$ (LB DRY AIR/LB FUEL)

EMISSIONS G/HR G/HP-HR CONC.

$\begin{array}{lrrr}\text { HC } & 347 . & .60 & 90 . \text { PPMC } \\ \text { CO } & 432 . & .74 & 57 . \text { PPM } \\ \text { NOX } & 5235 . & 8.98 & 421 . \text { PPM (D) } \\ \text { NOX, CORR } & 5721 . & 9.81 & \\ \text { CO2 } & & 561 . & 2.75 \text { PCT } \\ 02 & & & 17.15 \text { PCT }\end{array}$

CORRECTION FACTOR SUMMARY:

$\begin{array}{lllll}\text { EPA NOX CF }=1.0928 & \text { NOX-KH } & =1.0757 & \text { NOX-KT } & =1.0000 \\ \text { WET EXH HC CF }=.9705 & \text { AIR TEMP CF }=.9932 & \text { BAROM CF } & =1.0020 \\ \text { FUEL TEMP CF }=.9840 & \text { FUEL S.G. CF }=.9907 & \text { FUEL HHV CF } & =1.0062\end{array}$

PARTICULATE INFORMATION

FILTER PAIR I.D. NUMBER

FILTER PAIR WEIGHT GAIN (mg)

$6826.0 \cdot 111$

SAMPLE VOLUME (SCf)

4.578

38.138

.120

5.168

CO2-BASED DILUTION FACTOR

.620

PARTICULATE CONCENTRATION IN RAW EXHAUST (mI/scf)

146.

BRAKE-SPECIFIC PARTICULATE EMISSION RATE $(\mathrm{g} / \mathrm{hp}-\mathrm{hr})$ 


$\begin{array}{ll}\text { LOCOMOTIVE MODEL } & =\text { EMD GP38 } \\ \text { LOCOMOTIVE UNIT \# } & =\text { CSX \#2629 } \\ \text { THROTTLE NOTCH } & =\text { Notch } 4 \\ \text { TEST DATE } & =11 / 1 / 0 \\ \text { TEST NUMBER } & =6 / 10 \\ \text { SWRI FUEL CODE } & =\text { EM-2834-F } \\ \text { BAROMETER } & =29.09(\text { in. Hg) } \\ \text { DRY BULB TEMP } & =77 .\left({ }^{\circ}\right) \\ \text { ABS HUMIDITY } & =113.35(\text { GR/LB) }\end{array}$

ENGINE PERFORMANCE SUMMARY:

ACCESSORY HORSEPOWER:

$\begin{array}{rlr}\text { AUXILLARY GENERATOR HP } & =4.0 \\ \text { AIR COMPRESSOR HP } & =9.6 \\ \text { TRACTION MOTOR BLOWER HP } & =21.2 \\ \text { INERTIAL SEPERATOR BLOWER HP } & =3.2 \\ \text { RADIATOR FAN HP } & =9.8 \\ \text { TOTAL ACCESSORY HP } & =47.7\end{array}$

$\begin{array}{ll}\text { TEST LOCATION } & =\text { SWRI SA,TX } \\ \text { COMPUTER PROGRAM } & =\text { EPA.FOR } \\ \text { TEST CONFIGURATION } & =\text { CERT 100 } \\ \text { ENGINE MODEL } & =\text { EMD } 16-645 E 3 \\ \text { FUEL H/C RATIO } & =1.81 \\ \text { ENGINE INTAKE AIR } & =75 .\left(^{\circ} \mathrm{F}\right) \\ \text { WET BULB TEMP } & \left.=72 .{ }^{\circ} \mathrm{F}\right) \\ \text { RELATIVE HUMIDITY } & =79 .(\%)\end{array}$

394.0 (LB/HR)

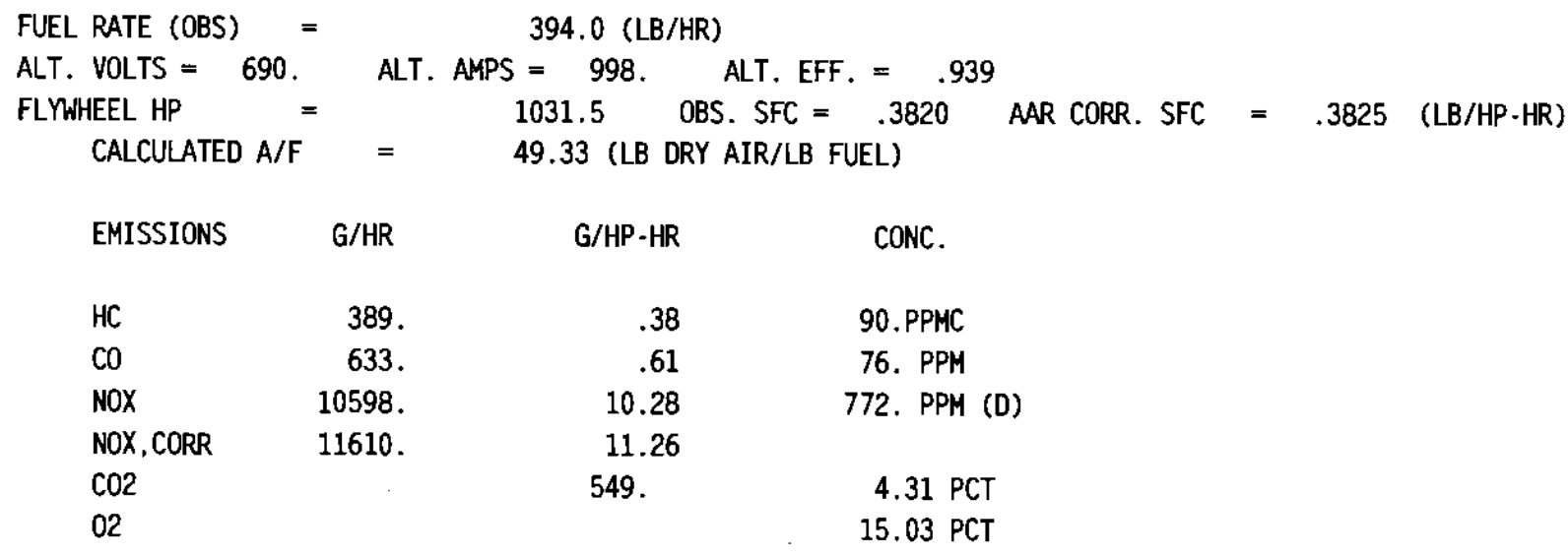

CORRECTION FACTOR SUMMARY:

$\begin{array}{lllll}\text { EPA NOX CF }=1.0954 & \text { NOX-KH } & =1.0779 & \text { NOX-KT } & =1.0000 \\ \text { WET EXH HC CF }=.9573 & \text { AIR TEMP CF }=.9932 & \text { 8AROM CF } & =1.0020 \\ \text { FUEL TEMP CF }=.9845 & \text { FUEL S.G. CF }=.9907 & \text { FUEL HHV CF } & =1.0062\end{array}$

PARTICULATE INFDRMATION

FILTER PAIR I.D. NUMBER FILTER PAIR WEIGHT GAIN (mg)

6B27.0-112

7.511

37.978

.198

6.771

1.339

353.

.343 


$\begin{array}{ll}\text { LOCOMOTIVE MODEL } & =\text { EMD GP38 } \\ \text { LOCOMOTIVE UNIT \# } & \text { CSX \#2629 } \\ \text { THROTTLE NOTCH } & =\text { Notch } 5 \\ \text { TEST DATE } & =11 / 1 / 0 \\ \text { TEST NUMBER } & =7 / 10 \\ \text { SWRI FUEL CODE } & =\text { EM-2834-F } \\ \text { BAROMETER } & =29.08(\mathrm{i} . \mathrm{Hg}) \\ \text { DRY BULB TEMP } & =76 .\left({ }^{\circ} \mathrm{F}\right) \\ \text { ABS HUMIDITY } & =113.66(\mathrm{GR} / \mathrm{LB})\end{array}$

ENGINE PERFORMANCE SUMMARY:

ACCESSORY HORSEPOWER:

$\begin{array}{rlr}\text { AUXILLARY GENERATOR HP } & = & 4.0 \\ \text { AIR COMPRESSOR HP } & = & 10.8 \\ \text { TRACTION MOTOR BLOWER HP } & = & 30.4 \\ \text { INERTIAL SEPERATOR BLOWER HP } & = & 4.6 \\ \text { RADIATOR FAN HP } & = & 28.1 \\ \text { TOTAL ACCESSORY HP } & =77.8\end{array}$

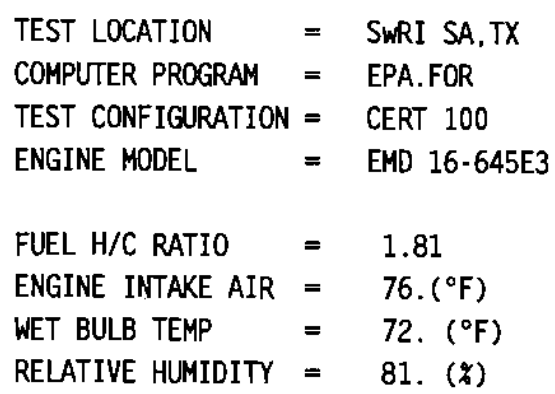

FUEL RATE (OBS) $\quad=\quad 496.0($ LB/HR)

ALT. VOLTS $=767 . \quad$ ALT. AMPS $=1104 . \quad$ ALT. EFF. $=.939$

FLYWHEEL HP $=1287.5$ OBS. SFC $=.3852$ AAR CORR. SFC $=.3856(\mathrm{LB} / \mathrm{HP}-\mathrm{HR})$

CALCULATED A/F $=45.50$ (LB DRY AIR/LB FUEL)

EMISSIONS G/HR G/HP-HR

$\begin{array}{lrrr}\text { HC } & 427 . & .33 & \text { 85. PPMC } \\ \text { CO } & 1001 . & .78 & 103 . \text { PPM } \\ \text { NOX } & 14033 . & 10.90 & 882 . \text { PPM (D) } \\ \text { NOX, CORR } & 15383 . & 11.95 & \\ \text { CO2 } & & 553 . & 4.68 \text { PCT } \\ 02 & & & 14.52 \text { PCT }\end{array}$

CORRECTION FACTOR SUMMARY:

$\begin{array}{lllll}\text { EPA NOX CF }=1.0962 & \text { NOX-KH } & =1.0785 & \text { NOX-KT } & =1.0000 \\ \text { WET EXH HC CF }=.9542 & \text { AIR TEMP CF }=.9928 & \text { BAROM CF } & =1.0019 \\ \text { FUEL TEMP CF }=.9845 & \text { FUEL S.G. CF }=.9907 & \text { FUEL HHV CF } & =1.0062\end{array}$

PARTICULATE INFORMATION

FILTER PAIR I.D. NUMBER

$6828.0-113$

FILTER PAIR WEIGHT GAIN (mg) 8.343

SAMPLE VOLUME (sCf)

37.400

DILUTE PM CONCENTRATION (mg/scf)

CO2-BASED DILUTION FACTOR 6.201

PARTICULATE CONCENTRATION IN RAW EXHAUST (mg/scf) 1.383

PARTICULATE MASS EMISSION RATE $(\mathrm{g} / \mathrm{hr})$

BRAKE-SPECIFIC PARTICULATE EMISSION RATE $(\mathrm{g} / \mathrm{hp}-\mathrm{hr}) \quad .330$ 
LOCOMOTIVE EMISSIONS SWRI PROJECT NO. 08-2999-001

$\begin{array}{ll}\text { LOCOMOTIVE MODEL } & =\text { EMD GP38 } \\ \text { LOCOMOTIVE UNIT \# } & =\text { CSX \#2629 } \\ \text { THROTTLE NOTCH } & =\text { Notch } 6 \\ \text { TEST DATE } & =11 / 1 / 0 \\ \text { TEST NUMBER } & =8 / 10 \\ \text { SWRI FUEL CODE } & =\text { EM-2834-F } \\ \text { BAROMETER } & =29.09(\mathrm{in} . \mathrm{Hg}) \\ \text { DRY BULB TEMP } & =76 .\left(^{\circ} \mathrm{F}\right) \\ \text { ABS HUMIDITY } & =112.44(\mathrm{GR} / \mathrm{LB})\end{array}$

ENGINE PERFORMANCE SUMMARY:

ACCESSORY HORSEPOWER:

$\begin{array}{rlr}\text { AUXILLARY GENERATOR HP } & =4.0 \\ \text { AIR COMPRESSOR HP } & =12.2 \\ \text { TRACTION MOTOR BLOWER HP } & =44.1 \\ \text { INERTIAL SEPERATOR BLOWER HP } & =6.6 \\ \text { RADIATOR FAN HP } & 40.7 \\ \text { TOTAL ACCESSORY HP } & =107.6\end{array}$

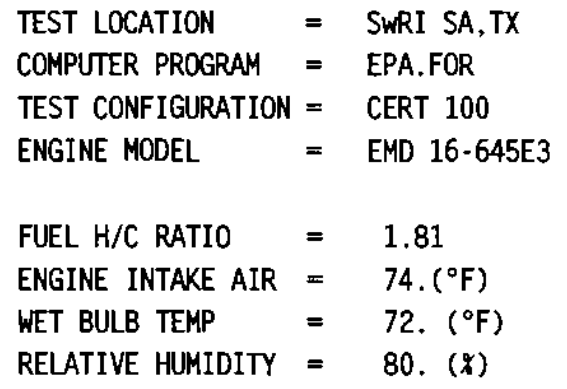

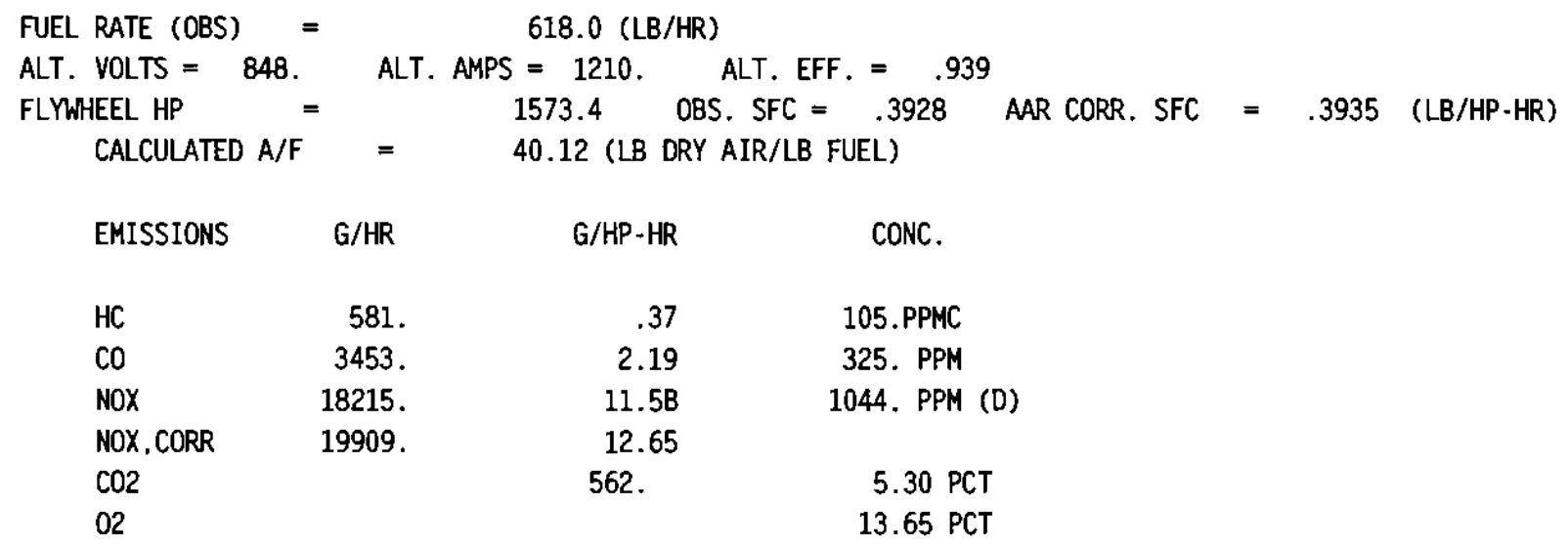

618.0 (LB/HR)

CORRECTION FACTOR SUMMARY:

$\begin{array}{lllll}\text { EPA NOX CF }=1.0930 & \text { NOX-KH } & =1.0759 & \text { NOX-KT } & =1.0000 \\ \text { WET EXH HC CF }=.9490 & \text { AIR TEMP CF }=.9937 & \text { BAROM CF } & =1.0020 \\ \text { FUEL TEMP CF }=.9855 & \text { FUEL S.G. CF }=.9907 & \text { FUEL HHV CF } & =1.0062\end{array}$

PARTICULATE INFORMATION

FILTER PAIR I.D. NUMBER

FILTER PAIR WEIGHT GAIN (mg)

$6829.0 \cdot 114$

7.499

37.120

SAMPLE VOLUME (SCf)

.202

DILUTE PM CONCENTRATION (mg/scf)

7.714

PARTICULATE CONCENTRATION IN RAW EXHAUST ( $\mathrm{mg} / \mathrm{scf}$ )

PARTICULATE MASS EMISSION RATE $(\mathrm{g} / \mathrm{hr})$

1.558

BRAKE-SPECIFIC PARTICULATE EMISSION RATE $(\mathrm{g} / \mathrm{hp}-\mathrm{hr})$

527.

.335 


$\begin{array}{ll}\text { LOCOMOTIVE MODEL } & =\text { EMD GP38 } \\ \text { LOCOMOTIVE UNIT \# } & =\text { CSX } \# 2629 \\ \text { THROTTLE NOTCH } & =\text { Notch } 7 \\ \text { TEST DATE } & =11 / 1 / 0 \\ \text { TEST NUMBER } & =9 / 10 \\ \text { SWRI FUEL CODE } & =\text { EM-2834-F } \\ \text { BAROMETER } & =29.09(\mathrm{in} . \mathrm{Hg}) \\ \text { DRY BULB TEMP } & =77 .\left({ }^{\circ} \mathrm{F}\right) \\ \text { ABS HUMIDITY } & =110.91(\mathrm{GR} / \mathrm{LB})\end{array}$

ENGINE PERFORMANCE SUMMARY:

ACCESSORY HORSEPOWER:

$\begin{array}{rlr}\text { AUXILLARY GENERATOR HP } & = & 4.0 \\ \text { AIR COMPRESSOR HP } & =13.8 \\ \text { TRACTION MOTOR BLOWER HP } & =63.5 \\ \text { INERTIAL SEPERATOR BLOWER HP } & =9.5 \\ \text { RADIATOR FAN HP } & =58.8 \\ \text { TOTAL ACCESSORY HP } & =149.6\end{array}$

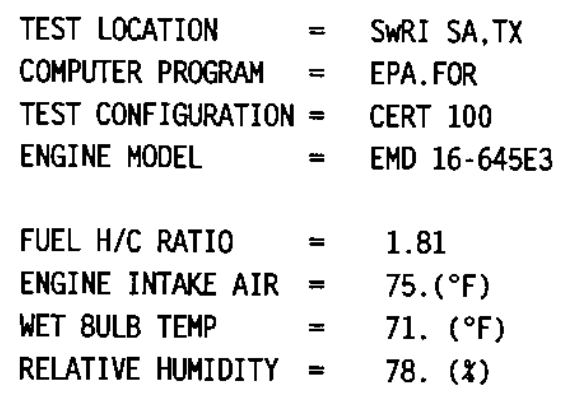

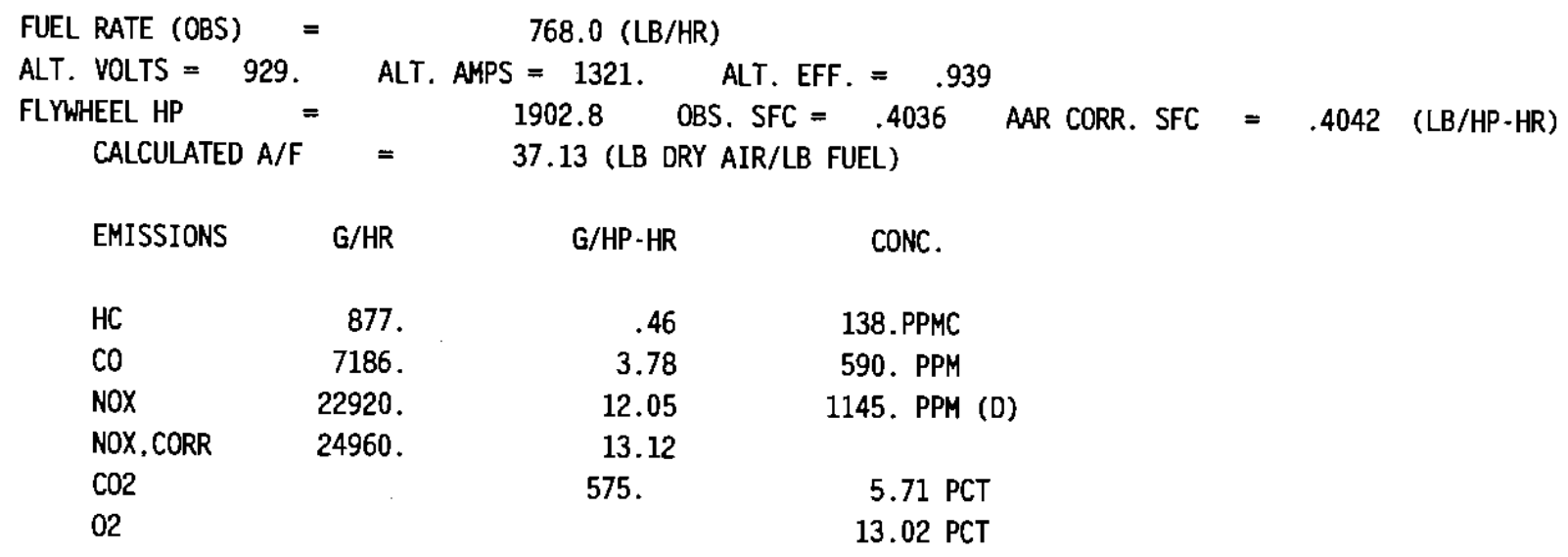

$768.0(\mathrm{LB} / \mathrm{HR})$

CORRECTION FACTOR SUMMARY:

$\begin{array}{lllll}\text { EPA NOX CF }=1.0890 & \text { NOX-KH } & =1.0727 & \text { NOX-KT } & =1.0000 \\ \text { WET EXH HC CF }=.9456 & \text { AIR TEMP CF }=.9932 & \text { BAROM CF } & =1.0020 \\ \text { FUEL TEMP CF }=.9860 & \text { FUEL S.G. CF }=.9907 & \text { FUEL HHV CF } & =1.0062\end{array}$

PARTICULATE INFORMATION

FILTER PAIR I.D. NUMBER $6830.0 \cdot 115$

FILTER PAIR WEIGHT GAIN (mg)

9.949

SAMPLE VOLUME (SCf)

36.758

DILUTE PM CONCENTRATION (mg/scf)

.271

7.801

CO2-BASED DILUTION FACTOR

2.112

PARTICULATE CONCENTRATION IN RAW EXHAUST (mg/scf)

823.

BRAKE-SPECIFIC PARTICULATE EMISSION RATE $(\mathrm{g} / \mathrm{hp}-\mathrm{hr})$ 


$\begin{array}{ll}\text { LOCOMOTIVE MODEL } & =\text { EMD GP38 } \\ \text { LOCOMOTIVE UNIT \# } & =\text { CSX } \# 2629 \\ \text { THROTTLE NOTCH } & =\text { Notch } 8 \\ \text { TEST DATE } & =11 / 1 / 0 \\ \text { TEST NUMBER } & =10 / 10 \\ \text { SWRI FUEL CODE } & =\text { EM-2834-F } \\ \text { BAROMETER } & =29.09(\mathrm{in} . \mathrm{Hg}) \\ \text { DRY BULB TEMP } & =76 .\left(^{\circ} \mathrm{F}\right) \\ \text { ABS HUMIDITY } & =111.08(\mathrm{GR} / \mathrm{LB})\end{array}$

ENGINE PERFORMANCE SUMMARY:

ACCESSORY HORSEPOWER:

$\begin{array}{rrr}\text { AUXILLARY GENERATOR HP } & = & 4.0 \\ \text { AIR COMPRESSOR HP } & =15.1 \\ \text { TRACTION MOTOR BLOWER HP } & =83.9 \\ \text { INERTIAL SEPERATOR BLOWER HP } & =12.6 \\ \text { RADIATOR FAN HP } & 77.6 \\ \text { TOTAL ACCESSORY HP } & =193.1\end{array}$

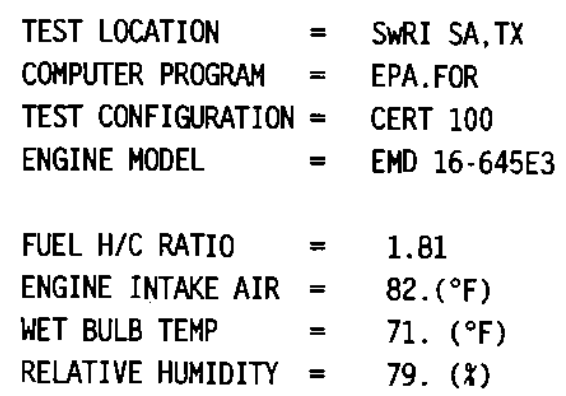

$912.0(\mathrm{LB} / \mathrm{HR})$

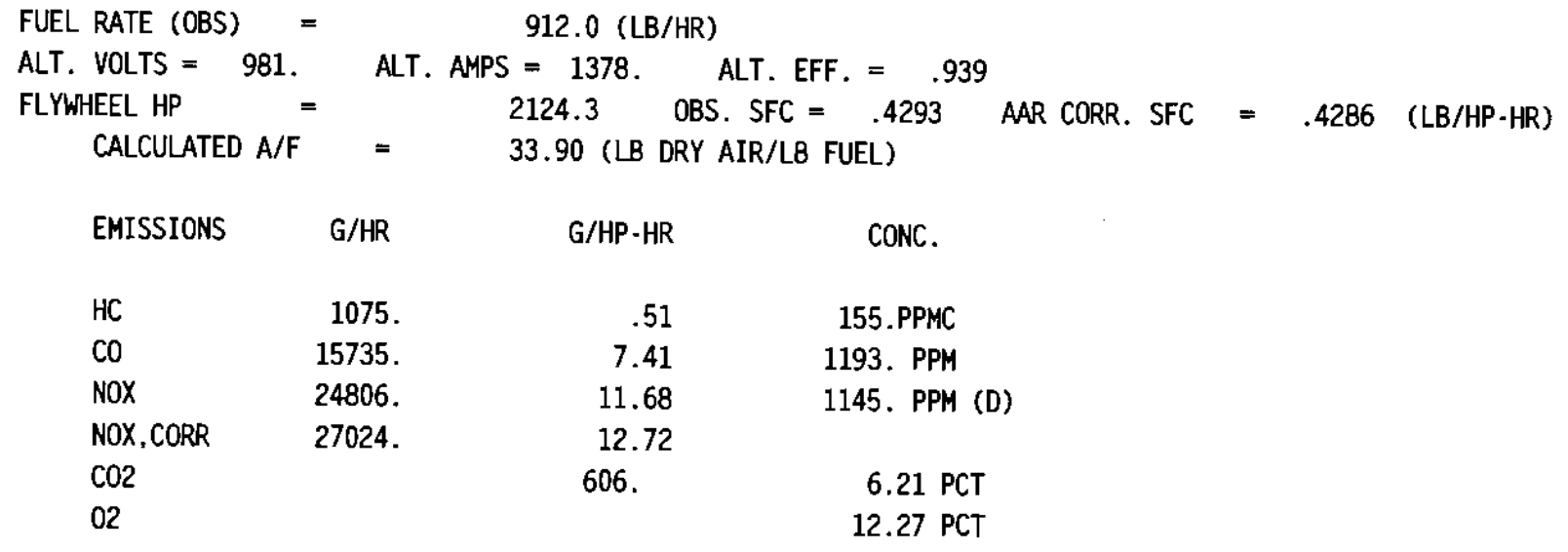

CORRECTION FACTOR SUMMARY:

$\begin{array}{lllll}\text { EPA NOX CF }=1.0894 & \text { NOX-KH } & =1.0730 & \text { NOX-KT } & =1.0000 \\ \text { WET EXH HC CF }=.9412 & \text { AIR TEMP CF }=.9901 & \text { BAROM CF } & =1.0020 \\ \text { FUEL TEMP CF }=.9860 & \text { FUEL S.G. CF }=.9907 & \text { FUEL HHV CF } & =1.0062\end{array}$

PARTICULATE INFORMATION

.......................

FILTER PAIR I.D. NUMBER

FILTER PAIR WEIGHT GAIN (mg)

$6831.0 \cdot 116$

SAMPLE VOLUME (scf)

12.241

35.866

DILUTE PM CONCENTRATION (mg/scf)

.341

CO2-BASED DILUTION FACTOR

7.377

2.518

PARTICULATE MASS EMISSION RATE $(g / h r)$

1067.

BRAKE-SPECIFIC PARTICULATE EMISSION RATE $(g / h p-h r)$

.502 


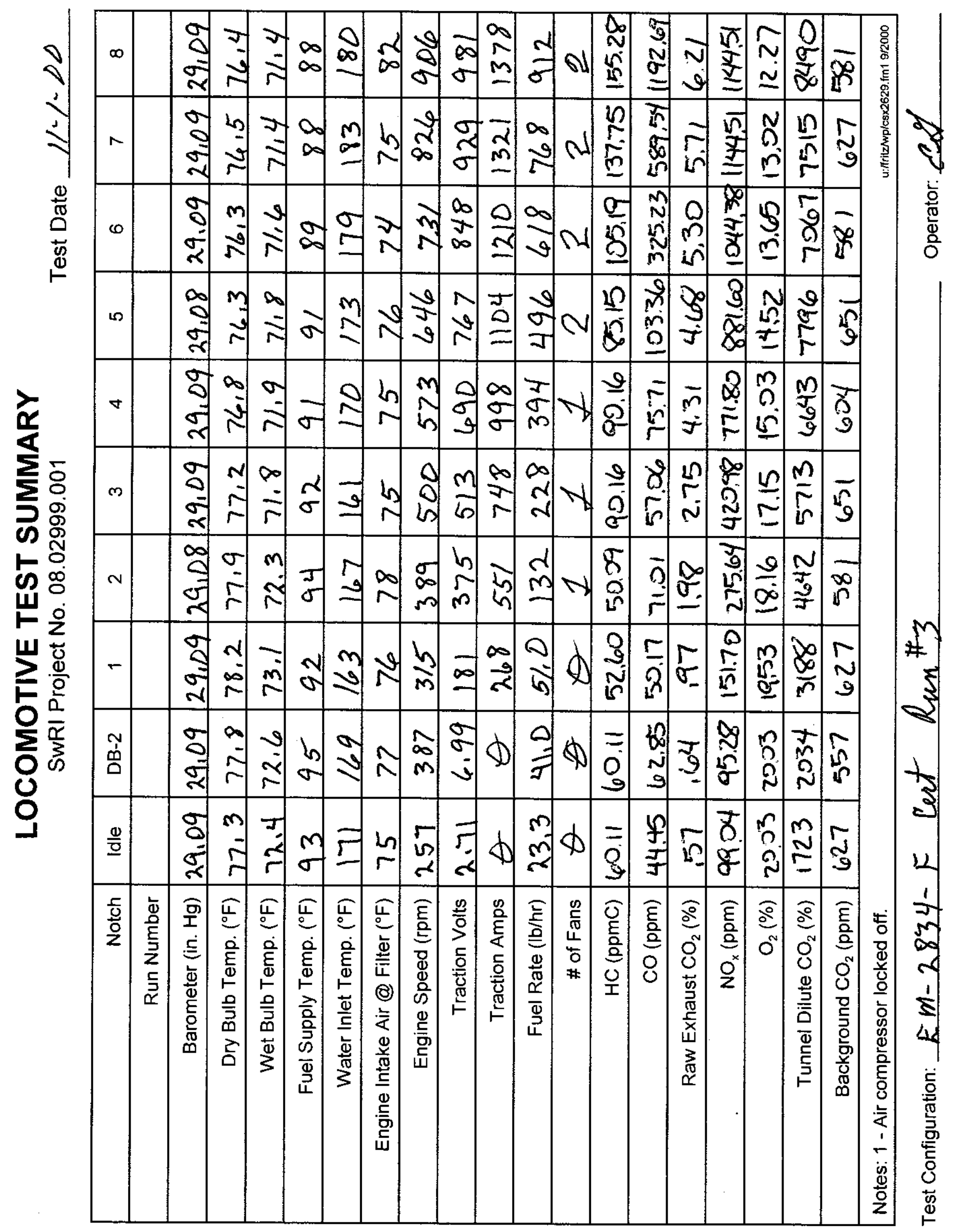




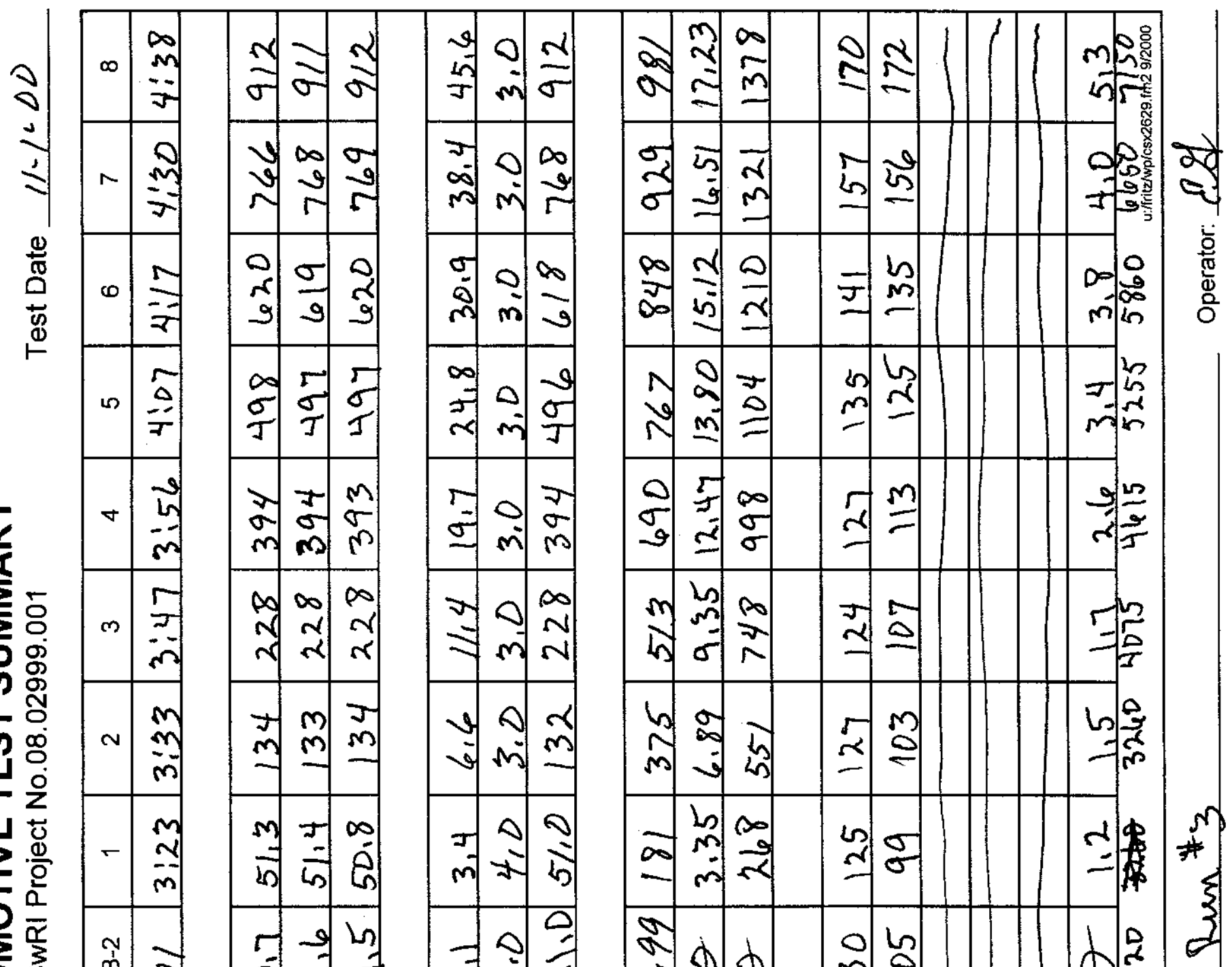

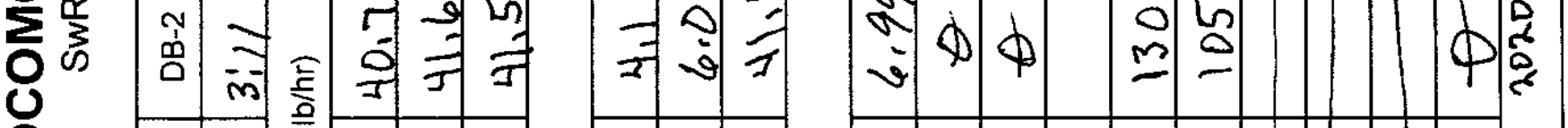

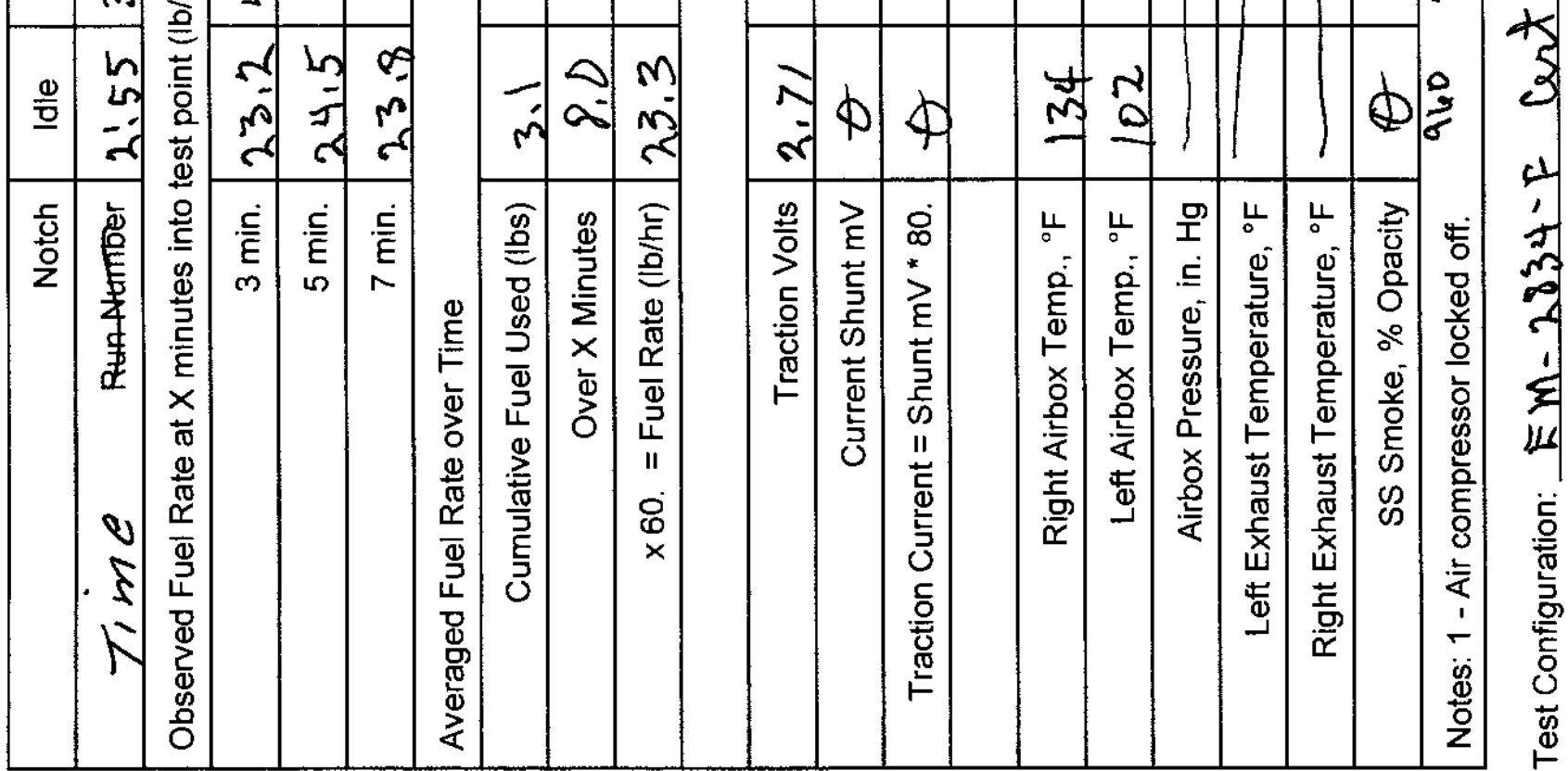




\section{APPENDIX B}

\section{CARB FUEL TEST RESULTS}




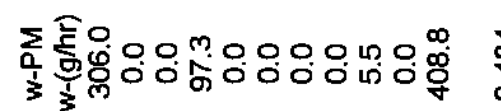

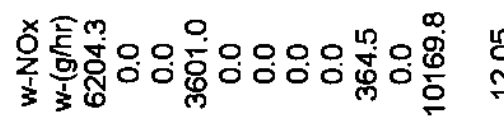

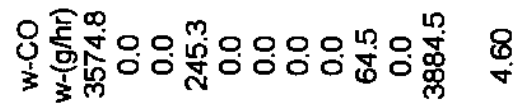

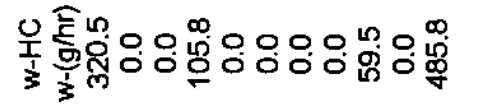

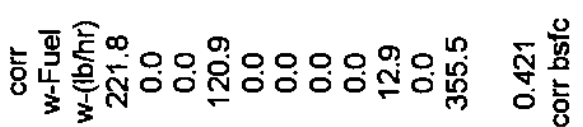

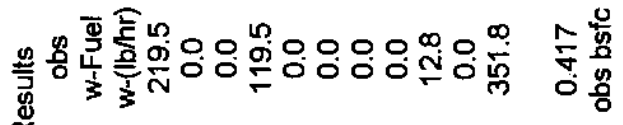

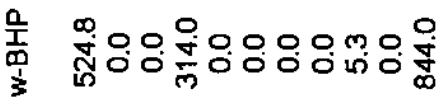

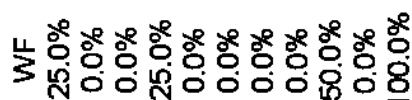

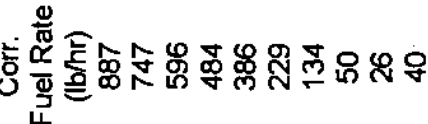

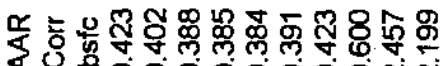

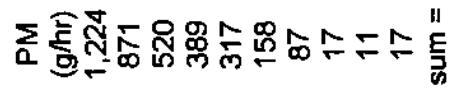

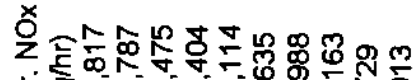

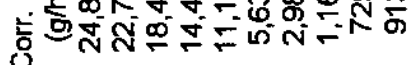

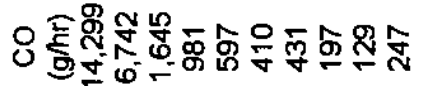

承

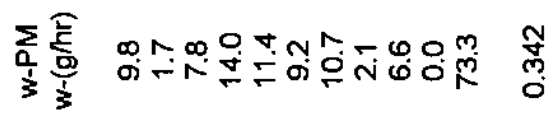

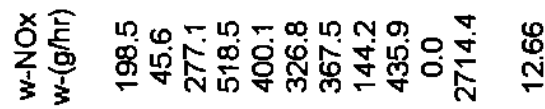

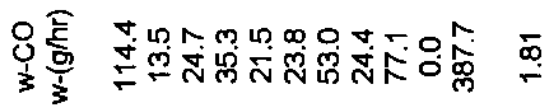

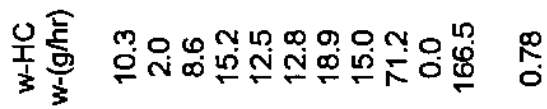

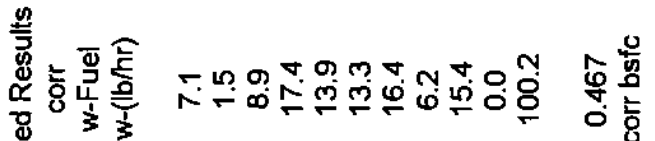

壳
亩落

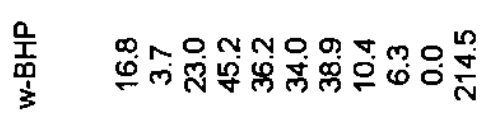

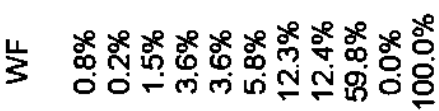

$\sum_{\frac{1}{3}} \frac{1}{5}$

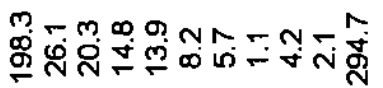

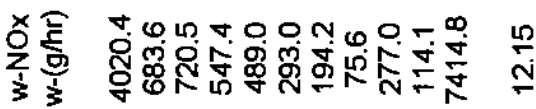

옿

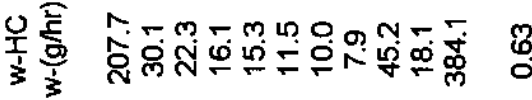

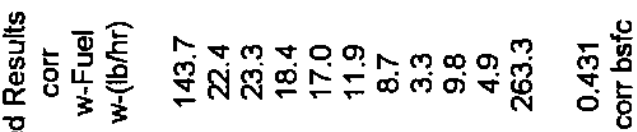

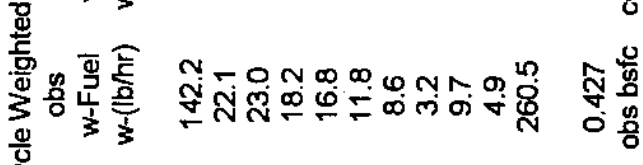

总

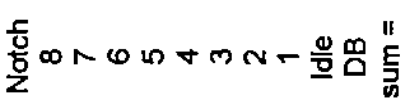




$\begin{array}{ll}\text { LOCOMOTIVE MODEL } & =\text { EMD GP38 } \\ \text { LOCOMOTIVE UNIT \# } & \text { CSX \#2629 } \\ \text { THROTTLE NOTCH } & =\text { IDLE } \\ \text { TEST DATE } & =10 / 27 / 0 \\ \text { TEST NUM8ER } & =1 / 10 \\ \text { SWRI FUEL CODE } & =\text { EM-2663.F } \\ \text { BAROMETER } & =29.25(\mathrm{in} . \mathrm{Hg}) \\ \text { DRY BULB TEMP } & =81 .\left({ }^{\circ} \mathrm{F}\right) \\ \text { ABS HUMIDITY } & =110.20(\mathrm{GR} / \mathrm{LB})\end{array}$

ENGINE PERFORMANCE SUMMARY:

ACCESSORY HORSEPOWER:

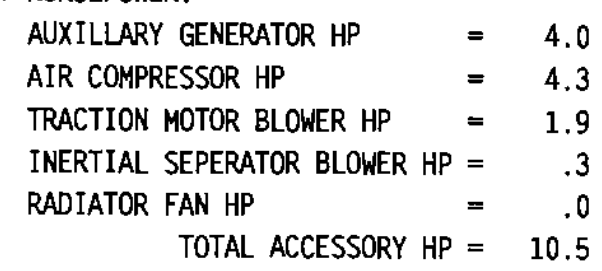

$\begin{array}{rlr}\text { AUXILLARY GENERATOR HP } & = & 4.0 \\ \text { AIR COMPRESSOR HP } & = & 4.3 \\ \text { TRACTION MOTOR BLOWER HP } & =1.9 \\ \text { INERTIAL SEPERATOR BLOWER HP } & = & .3 \\ \text { RADIATOR FAN HP } & =.0 \\ \text { TOTAL ACCESSORY HP } & =10.5\end{array}$

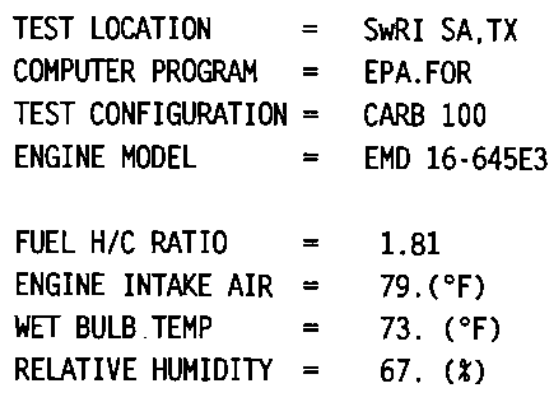

CORRECTION FACTOR SUMMARY:

EPA NOX CF $=1.0995 \quad$ NOX-KH $=1.0812 \quad$ NOX-KT $=1.0000$

WET EXH HC CF $=.9891$ AIR TEMP CF $=.9914$ BAROM CF $=1.0034$

FUEL TEMP CF $=.9815 \quad$ FUEL S.G. CF $=1.0187$ FUEL HHV CF $=1.0189$

PARTICULATE INFORMATION

FILTER PAIR I.D. NUMBER

$6742.0-27$

FILTER PAIR WEIGHT GAIN (mg)

.718

39.552

.018

DILUTE PM CONCENTRATION (mg/scf)

CO2-BASED DILUTION FACTOR

PARTICULATE CONCENTRATION IN RAW EXHAUST (mg/scf)

PARTICULATE MASS EMISSION RATE $(\mathrm{g} / \mathrm{hr})$

BRAKE-SPECIFIC PARTICULATE EMISSION RATE ( $g / h p-h r)$
5.560

.101

11.

1.071 


$\begin{array}{llll}\text { LOCOMOTIVE MODEL } & =\text { EMD GP38 } & \text { TEST LOCATION } & =\text { SWRI SA, TX } \\ \text { LOCOMOTIVE UNIT \# } & =\text { CSX \#2629 } & \text { COMPUTER PROGRAM } & =\text { EPA.FOR } \\ \text { THROTTLE NOTCH } & =\text { D82 } & \text { TEST CONFIGURATION } & \text { CARB 100 } \\ \text { TEST DATE } & =10 / 27 / 0 & \text { ENGINE MODEL } & =\text { EMD } 16-645 E 3 \\ \text { TEST NUMBER } & =2 / 10 & & \\ \text { SWRI FUEL CODE } & =\text { EM-2663-F } & \text { FUEL H/C RATIO } & =1.81 \\ \text { BAROMETER } & =29.24(\mathrm{in} . \mathrm{Hg}) & \text { ENGINE INTAKE AIR } & =81 .\left({ }^{\circ} \mathrm{F}\right) \\ \text { DRY BULB TEMP } & =85 .\left({ }^{\circ} \mathrm{F}\right) & \text { WET BULB TEMP } & =73 .\left({ }^{\circ} \mathrm{F}\right) \\ \text { ABS HUMIDITY } & =105.77(\mathrm{GR} / \mathrm{LB}) & \text { RELATIVE HUMIDITY } & =57 .(\%)\end{array}$

ENGINE PERFORMANCE SUMMARY:

ACCESSORY HORSEPOWER:

$\begin{array}{rlr}\text { AUXILLARY GENERATOR HP } & = & 4.0 \\ \text { AIR COMPRESSOR HP } & = & 6.5 \\ \text { TRACTION MOTOR BLOWER HP } & =6.5 \\ \text { INERTIAL SEPERATOR BLOWER HP } & =1.0 \\ \text { RADIATOR FAN HP } & .0 \\ \text { TOTAL ACCESSORY HP } & =18.0\end{array}$

FUEL RATE (OBS) =

ALT. VOLTS $=$

FLYWHEEL HP

B.

CALCULATED A/F

EMISSIONS

G/HR

G/HP.HR

8.07

13.75

46.79

50.83

3085 .

$\mathrm{CO} 2$

02

CORRECTION FACTOR SUMMARY:

EPA NOX CF $=1.0862$

WET EXH HC CF $=.9887$

FUEL TEMP CF $=.9830$

$\begin{array}{ll}\text { NOX-KH } & =1.0704 \\ \text { AIR TEMP CF } & =.9905\end{array}$

FUEL S.G. $C F=1.0187$

PARTICULATE INFORMATION

FILTER PAIR I.D. NUMBER

FILTER PAIR WEIGHT GAIN (mg)

SAMPLE VOLUME (SCf)

DILUTE PM CONCENTRATION (mg/scf)

CO2-BASED DILUTION FACTOR

PARTICULATE CONCENTRATION IN RAW EXHAUST (mg/scf)

PARTICULATE MASS EMISSION RATE $(g / \mathrm{hr})$

BRAKE-SPECIFIC PARTICULATE EMISSION RATE $(g / h p-h r)$

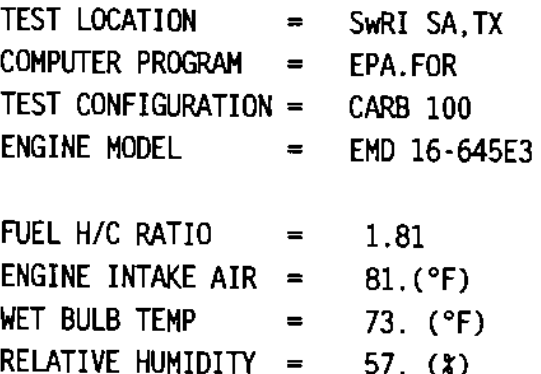




$\begin{array}{ll}\text { LOCOMOTIVE MODEL } & =\text { EMD GP38 } \\ \text { LOCOMOTIVE UNIT \# } & =\text { CSX \#2629 } \\ \text { THROTTLE NOTCH } & =\text { Notch } 1 \\ \text { TEST DATE } & =10 / 27 / 0 \\ \text { TEST NUMBER } & =3 / 10 \\ \text { SWRI FUEL CODE } & =\text { EM-2663.F } \\ \text { BAROMETER } & =29.24(\mathrm{in} . \mathrm{Hg}) \\ \text { DRY BULB TEMP } & =82 .\left(^{\circ} \mathrm{F}\right) \\ \text { ABS HUMIDITY } & =105.64(\mathrm{GR} / \mathrm{LB})\end{array}$

ENGINE PERFORMANCE SUMMARY:

ACCESSORY HORSEPOWER:

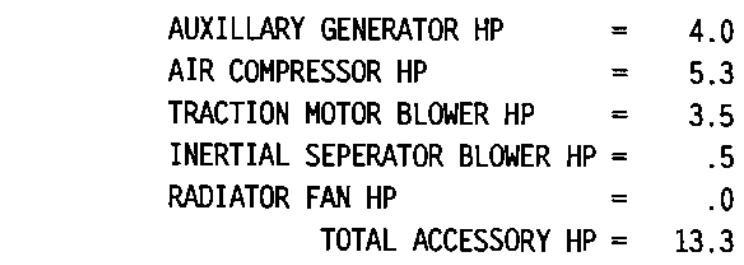

$\begin{array}{rlr}\text { AUXILLARY GENERATOR HP } & =4.0 \\ \text { AIR COMPRESSOR HP } & 5.3 \\ \text { TRACTION MOTOR BLOWER HP } & =3.5 \\ \text { INERTIAL SEPERATOR BLOWER HP } & =.5 \\ \text { RADIATOR FAN HP } & .0 \\ \text { TOTAL ACCESSORY HP } & =13.3\end{array}$

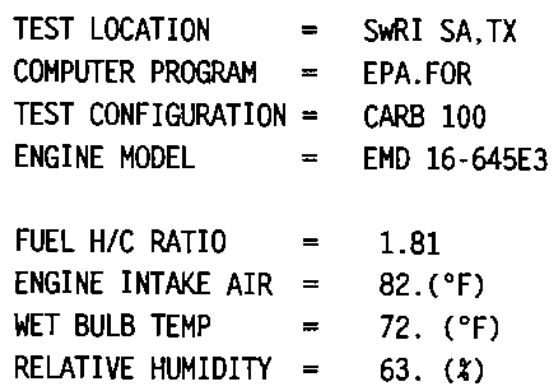

CORRECTION FACTOR SUMMARY:

$\begin{array}{lllll}\text { EPA NOX CF }=1.0831 & \text { NOX-KH } & =1.0679 & \text { NOX-KT } & =1.0000 \\ \text { WET EXH HC CF }=.9854 & \text { AIR TEMP CF }=.9901 & \text { BAROM CF } & =1.0033 \\ \text { FUEL TEMP CF }=.9835 & \text { FUEL S.G. CF }=1.0187 & \text { FUEL HHV CF } & =1.0189\end{array}$

\section{PARTICULATE INFORMATION}

FILTER PAIR I.D. NUMBER

$6744.0-29$
1.364
38.248
.036
3.651
.130
17.

FILTER PAIR WEIGHT GAIN (mg)

SAMPLE VOLUME (SCf)

DILUTE PM CONCENTRATION (mg/scf)

CO2-BASED DILUTION FACTOR

PARTICULATE CONCENTRATION IN RAW EXHAUST (mg/scf)

PARTICULATE MASS EMISSION RATE $(g / h r)$

17.

8RAKE-SPECIFIC PARTICULATE EMISSION RATE $(\mathrm{g} / \mathrm{hp}-\mathrm{hr})$

.201 


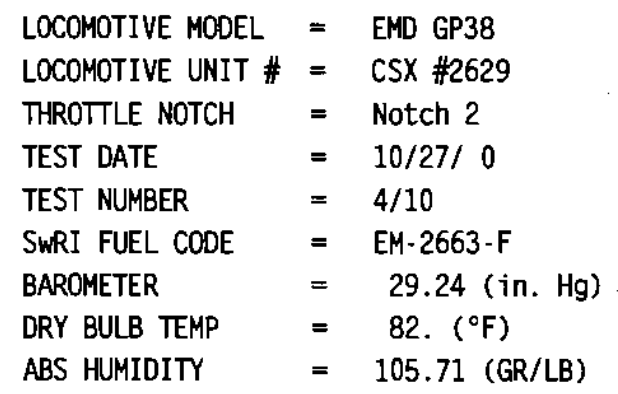

ENGINE PERFORMANCE SUMMARY:

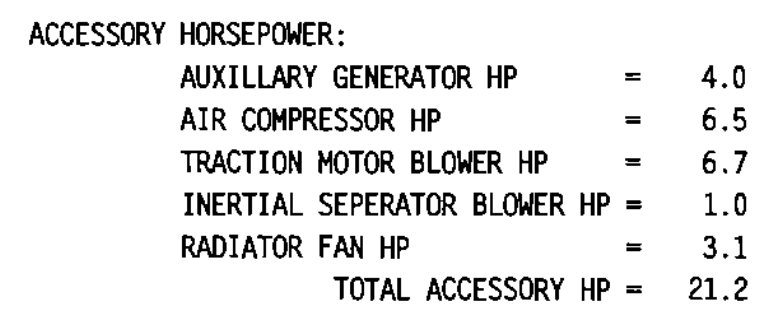

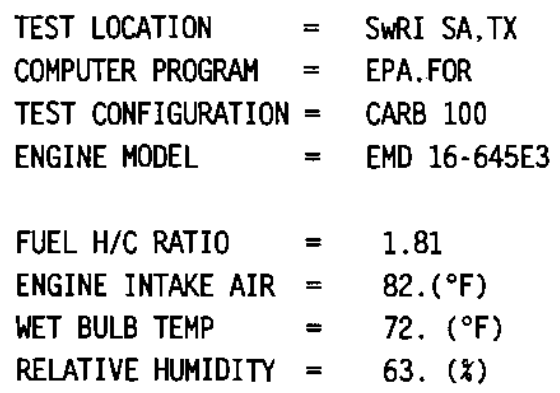




$\begin{array}{ll}\text { LOCOMOTIVE MODEL } & =\text { EMD GP38 } \\ \text { LOCOMOTIVE UNIT \# } & =\text { CSX } 2629 \\ \text { THROTTLE NOTCH } & =\text { Notch } 3 \\ \text { TEST DATE } & =10 / 27 / 0 \\ \text { TEST NUMBER } & =5 / 10 \\ \text { SWRI FUEL CODE } & =\text { EM-2663-F } \\ \text { BAROMETER } & =29.23(\mathrm{in} . \mathrm{Hg}) \\ \text { DRY BULB TEMP } & =83 .\left(^{\circ} \mathrm{F}\right) \\ \text { ABS HUMIDITY } & =105.90(\mathrm{GR} / \mathrm{LB})\end{array}$

ENGINE PERFORMANCE SUMMARY:

ACCESSORY HORSEPOWER:

$\begin{array}{rlr}\text { AUXILLARY GENERATOR HP } & =4.0 \\ \text { AIR COMPRESSOR HP } & =8.3 \\ \text { TRACTION MOTOR BLOWER HP } & =14.0 \\ \text { INERTIAL SEPERATOR BLOWER HP } & =2.1 \\ \text { RADIATOR FAN HP } & 6.5 \\ \text { TOTAL ACCESSORY HP } & =34.9\end{array}$

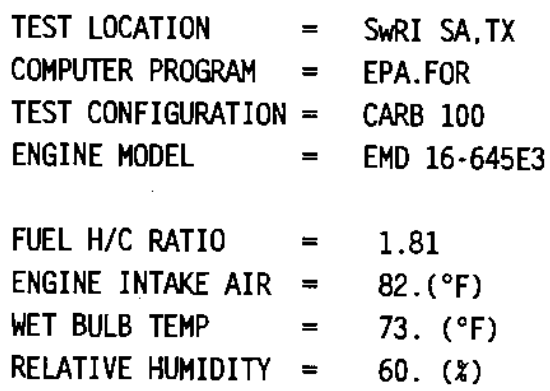

226.0 (LB/HR)

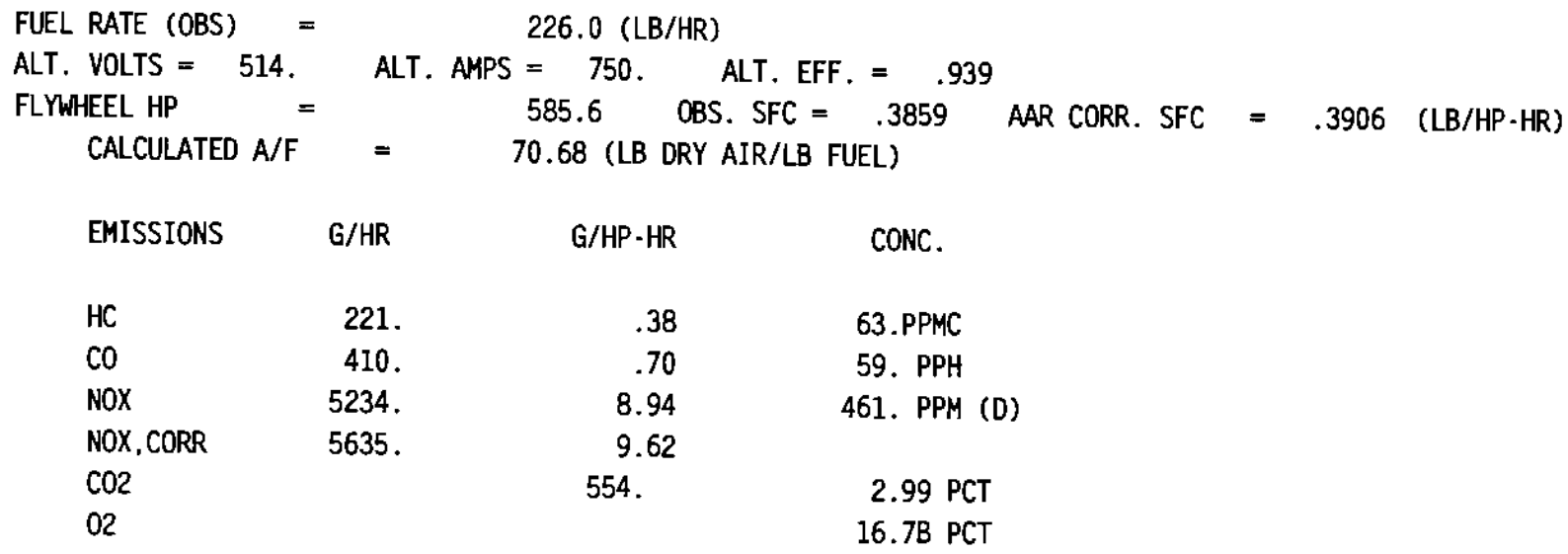

CORRECTION FACTOR SUMMARY:

$\begin{array}{lllll}\text { EPA NOX CF }=1.0767 & \text { NOX-KH } & =1.0626 & \text { NOX-KT } & =1.0000 \\ \text { WET EXH HC CF }=.9687 & \text { AIR TEMP CF }=.9901 & \text { BAROM CF } & =1.0032 \\ \text { FUEL TEMP CF }=.9835 & \text { FUEL S.G. CF }=1.0187 & \text { FUEL HHV CF } & =1.0189\end{array}$

PARTICULATE INFORMATION

FILTER PAIR I.D. NUMBER

$6746.0-31$

5.529

38.105

.145

5.040

.731

158.

.269 


$\begin{array}{ll}\text { LOCOMOTIVE MODEL } & =\text { EMD GP38 } \\ \text { LOCOMOTIVE UNIT \# } & =\text { CSX \#2629 } \\ \text { THROTTLE NOTCH } & =\text { Notch } 4 \\ \text { TEST DATE } & =10 / 27 / 0 \\ \text { TEST NUMBER } & =6 / 10 \\ \text { SWRI FUEL CODE } & =\text { EM-2663-F } \\ \text { BAROMETER } & =29.22(\text { in. Hg) } \\ \text { DRY BULB TEMP } & =86 .\left(^{\circ} \mathrm{F}\right) \\ \text { ABS HUMIDITY } & =103.37(\mathrm{GR} / \mathrm{LB})\end{array}$

ENGINE PERFORMANCE SUMMARY:

$\begin{array}{ll}\text { TEST LOCATION } & =\text { SWRI SA,TX } \\ \text { COMPUTER PROGRAM } & =\text { EPA. FOR } \\ \text { TEST CONFIGURATION } & =\text { CARB } 100 \\ \text { ENGINE MODEL } & =\text { EMD } 16-645 E 3 \\ \text { FUEL H/C RATIO } & =1.81 \\ \text { ENGINE INTAKE AIR } & =83 .\left({ }^{\circ} \mathrm{F}\right) \\ \text { WET BULB TEMP } & =73 .\left({ }^{\circ} \mathrm{F}\right) \\ \text { RELATIVE HUMIDITY } & =54 .\left({ }^{\circ}\right)\end{array}$

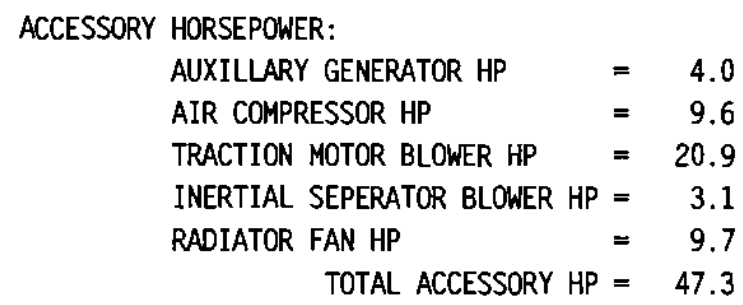

FILTER PAIR I.D. NUMBER

6747.0-32

7.907

38.067

.208

6.127

1.273

317.

.315 


$\begin{array}{ll}\text { LOCOMOTIVE MODEL } & =\text { EMD GP38 } \\ \text { LOCOMOTIVE UNIT \# } & =\text { CSX \#2629 } \\ \text { THROTLLE NOTCH } & =\text { Notch } 5 \\ \text { TEST DATE } & =10 / 27 / 0 \\ \text { TEST NUMBER } & =7 / 10 \\ \text { SWRI FUEL CODE } & =\text { EM-2663-F } \\ \text { BAROMETER } & =29.22(\mathrm{in} . \mathrm{Hg}) \\ \text { DRY BULB TEMP } & =85 .\left({ }^{\circ} \mathrm{F}\right) \\ \text { ABS HUMIDITY } & =105.09(\mathrm{GR} / \mathrm{LB})\end{array}$

ENGINE PERFORMANCE SUMMARY:

\section{ACCESSORY HORSEPOWER:}

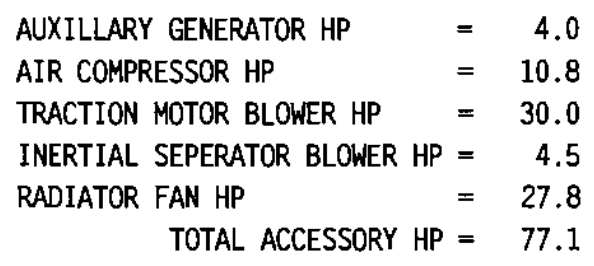




$\begin{array}{ll}\text { LOCOMOTIVE MODEL } & =\text { EMD GP38 } \\ \text { LOCOMOTIVE UNIT \# } & =\text { CSX \#2629 } \\ \text { THROTLE NOTCH } & =\text { Notch } 6 \\ \text { TEST DATE } & =10 / 27 / 0 \\ \text { TEST NUMBER } & =8 / 10 \\ \text { SWRI FUEL CODE } & =\text { EM-2663-F } \\ \text { BAROMETER } & =29.21(\text { in. Hg) } \\ \text { DRY BULB TEMP } & =86 .\left({ }^{\circ} \mathrm{F}\right) \\ \text { ABS HUMIDITY } & =102.98(\mathrm{GR} / \mathrm{LB})\end{array}$

ENGINE PERFORMANCE SUMMARY:

ACCESSORY HORSEPOWER:

$\begin{array}{llr}\text { AUXILLARY GENERATOR HP } & = & 4.0 \\ \text { AIR COMPRESSOR HP } & = & 12.2 \\ \text { TRACTION MOTOR BLOWER HP } & = & 43.4 \\ \text { INERTIAL SEPERATOR BLOWER HP } & 6.5 \\ \text { RADIATOR FAN HP } & =40.2\end{array}$

TOTAL ACCESSORY HP $=106.3$

$\begin{array}{ll}\text { TEST LOCATION } & =\text { SWRI SA.TX } \\ \text { COMPUTER PROGRAM } & =\text { EPA.FOR } \\ \text { TEST CONFIGURATION } & =\text { CARB } 100 \\ \text { ENGINE MODEL } & =\text { EMD } 16.645 E 3 \\ \text { FUEL H/C RATIO } & =1.81 \\ \text { ENGINE INTAKE AIR } & =85 .\left({ }^{\circ} \mathrm{F}\right) \\ \text { WET BULB TEMP } & =73 .\left({ }^{\circ} \mathrm{F}\right) \\ \text { RELATIVE HUMIDITY } & =54 .(\%)\end{array}$

FUEL RATE (OBS) $=590.0$ (LB/HR)

ALT. VOLTS $=835 . \quad$ ALT. AMPS $=1198 . \quad$ ALT. EFF. $=.939$

FLYWHEEL HP $=1535.4$ OBS. SFC $=.3843 \quad$ AAR CORR. SFC $=.3883(\mathrm{LB} / \mathrm{HP} \cdot \mathrm{HR})$

CALCULATED A/F $=38.54$ (LB DRY AIR/LB FUEL)

$\begin{array}{llll}\text { EMISSIONS } & G / H R & G / H P \cdot H R & \text { CONC. }\end{array}$

$\begin{array}{lrrr}\text { HC } & 572 . & .37 & 113 . \text { PPMC } \\ \text { CO } & 1645 . & 1.07 & 169 . \text { PPM } \\ \text { NOX } & 17287 . & 11.26 & 1082 . \text { PPM (D) } \\ \text { NOX, CORR } & 18475 . & 12.03 & \\ \text { CO2 } & & 551 . & 5.54 \text { PCT } \\ 02 & & & 13.27 \text { PCT }\end{array}$

CORRECTION FACTOR SUMMARY:

$\begin{array}{lllll}\text { EPA NOX CF }=1.0688 & \text { NOX-KH } & =1.0562 & \text { NOX-KT } & =1.0000 \\ \text { WET EXH HC CF }=.9476 & \text { AIR TEMP CF }=.9887 & \text { 8AROM CF } & =1.0030 \\ \text { FUEL TEMP CF }=.9870 & \text { FUEL S.G. CF }=1.0187 & \text { FUEL HHV CF } & =1.0189\end{array}$

PARTICULATE 1NFORMATION

FILTER PAIR I.D. NUMBER

$6749.0 \cdot 34$

FILTER PAIR WEIGHT GAIN (mg) $\quad 8.470$

SAMPLE VOLUME (SCf)

37.125

DILUTE PM CONCENTRATION (mg/scf)

.228

7.336

CO2-BASED DILUTION FACTOR

1.674

PARTICULATE CONCENTRATION IN RAW EXHAUST (mg/scf)

520.

BRAKE-SPECIFIC PARTICULATE EMISSION RATE $(\mathrm{g} / \mathrm{hp}-\mathrm{hr})$ 


$\begin{array}{ll}\text { LOCOMOTIVE MODEL } & =\text { EMD GP38 } \\ \text { LOCOMOTIVE UNIT \# } & \text { CSX } \# 2629 \\ \text { THROTTLE NOTCH } & =\text { Notch } 7 \\ \text { TEST DATE } & =10 / 27 / 0 \\ \text { TEST NUMBER } & =9 / 10 \\ \text { SWRI FUEL CODE } & =\text { EM-2663-F } \\ \text { BAROMETER } & =29.21(\mathrm{in} . \mathrm{Hg}) \\ \text { ORY BULB TEMP } & =86 .\left(^{\circ} \mathrm{F}\right) \\ \text { ABS HUMIDITY } & =101.27(\mathrm{GR} / \mathrm{LB})\end{array}$

ENGINE PERFORMANCE SUMMARY:

ACCESSORY HORSEPOWER:

$\begin{array}{llr}\text { AUXILLARY GENERATOR HP } & = & 4.0 \\ \text { AIR COMPRESSOR HP } & =13.8 \\ \text { TRACTION MOTOR BLOWER HP } & =62.7 \\ \text { INERTIAL SEPERATOR BLOWER HP } & =9.4 \\ \text { RADIATOR FAN HP } & 5 B .0 \\ \text { TOTAL ACCESSORY HP } & =147.9\end{array}$

FUEL RATE (OBS) =

738.0 (LB/HR)

ALT. VOLTS $=917$.

ALT. AMPS $=1306$.

ALT. EFF. $=.939$

FLYWHEEL HP =

CALCULATED A/

1858.8 OBS. $\mathrm{SFC}=\quad .3970$

34.64 (LB DRY AIR/LB FUEL)

EMISSIONS

G/HR

G/HP-HR

CONC.

$\mathrm{HC}$

$\mathrm{CO}$

NOX

1003.

.54

3.63

11.52

$2140 \mathrm{~B}$.

12.26

$\mathrm{CO} 2$

22787.

565 .

02

CORRECTION FACTOR SUMMARY:

EPA NOX CF $=1.0644$

NOX-KH $=1.0527$

AIR TEMP CF $=.9905$

NOX-KT $=1.0000$

WET EXH HC CF $=.9426$

FUEL S.G. CF $=1.0187$

BAROM CF $\quad=1.0030$

FUEL HHV CF $=1.0189$

12.40 PCT

175. PPMC

61B. PPM

1195. PPM (D)

$6.13 \mathrm{PCT}$

PARTICULATE INFORMATION

FILTER PAIR I.D. NUMBER

FILTER PAIR WEIGHT GAIN (mg)

$6750.0-35$

10.494

36.666

SAMPLE VOLUME (scf)

$.2 \mathrm{~B} 6$

8.692

$2.48 \mathrm{~B}$

PARTICULATE CONCENTRATION IN RAW EXHAUST (mg/scf)

PARTICULATE MASS EMISSION RATE $(\mathrm{g} / \mathrm{hr})$

871.

BRAKE-SPECIFIC PARTICULATE EMISSION RATE $(\mathrm{g} / \mathrm{hp}-\mathrm{hr})$

.469 


$\begin{array}{ll}\text { LOCOMOTIVE MODEL } & =\text { EMD GP38 } \\ \text { LOCOMOTIVE UNIT \# } & \text { CSX \#2629 } \\ \text { THROTLLE NOTCH } & =\text { Notch } 8 \\ \text { TEST DATE } & =10 / 27 / 0 \\ \text { TEST NUMBER } & =10 / 10 \\ \text { SWRI FUEL CODE } & =\text { EM-2663-F } \\ \text { BAROMETER } & =29.21(\text { in. Hg) } \\ \text { DRY BULB TEMP } & =84 .\left({ }^{\circ} \mathrm{F}\right) \\ \text { ABS HUMIDITY } & =98.02(\mathrm{GR} / \mathrm{LB})\end{array}$

ENGINE PERFORMANCE SUMMARY:

ACCESSORY HORSEPOWER:

$\begin{array}{rlr}\text { AUXILLARY GENERATOR HP } & = & 4.0 \\ \text { AIR COMPRESSOR HP } & = & 15.1 \\ \text { TRACTION MOTOR BLOWER HP } & = & 33.0 \\ \text { INERTIAL SEPERATOR BLOWER HP } & =12.4 \\ \text { RADIATOR FAN HP } & =76.7 \\ \text { TOTAL ACCESSORY HP } & =191.2\end{array}$

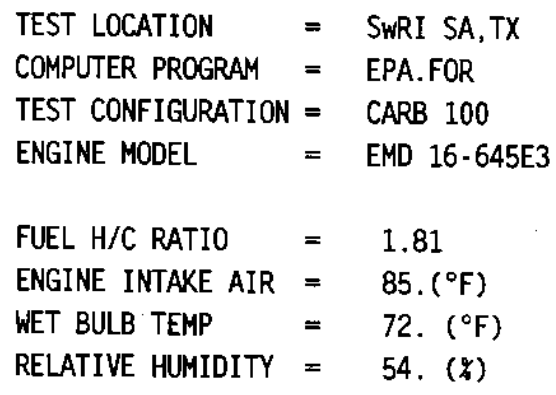

FUEL RATE (OBS) $=878.0$ (LB/HR)

ALT. VOLTS $=972 . \quad$ ALT. AMPS $=1374 . \quad$ ALT. EFF. $=.939$

FLYWHELL HP $=2099.1$ OBS. SFC $=.4183$ AAR CORR. SFC $=.4226(\mathrm{LB} / \mathrm{HP} \cdot \mathrm{HR})$

CALCULATED A/F $=31.69$ (LB DRY AIR/LB FUEL)

$\begin{array}{lll}\text { EMISSIONS G/HR G/HP-HR } & \text { CONC. }\end{array}$

$\begin{array}{lrrr}\text { HC } & 1282 . & .61 & 205 . \text { PPMC } \\ \text { CO } & 14299 . & 6.81 & 1207 . \text { PPM } \\ \text { NOX } & 23496 . & 11.19 & 1207 . \text { PPM (D) } \\ \text { NOX, CORR } & 24817 . & 11.82 & \\ \text { CO2 } & & 591 . & 6.66 \text { PCT } \\ 02 & & & 11.64 \text { PCT }\end{array}$

CORRECTION FACTOR SUMMARY:

$\begin{array}{lllll}\text { EPA NOX CF }=1.0562 & \text { NOX-KH } & =1.0460 & \text { NOX-KT } & =1.0000 \\ \text { WET EXH HC CF }=.9382 & \text { AIR TEMP CF }=.9887 & \text { BAROM CF } & =1.0030 \\ \text { FUEL TEMP CF }=.9850 & \text { FUEL S.G. CF }=1.0187 & \text { FUEL HHV CF } & =1.0189\end{array}$

PARTICULATE INFORMATION

FILTER PAIR I.D. NUMBER

$6751.0-36$

FILTER PAIR WEIGHT GAIN (mg) 15.611

SAMPLE VOLUME (sCf)

35.195

DILUTE PM CONCENTRATION (mg/scf)

CO2-BASED DILUTION FACTOR $\quad 7.223$

PARTICULATE CONCENTRATION IN RAW EXHAUST (mg/scf) 3.204

PARTICULATE MASS EMISSION RATE $(\mathrm{g} / \mathrm{hr}) \quad 1224$.

BRAKE-SPECIFIC PARTICULATE EMISSION RATE $(\mathrm{g} / \mathrm{hp}-\mathrm{hr}) \quad .583$ 


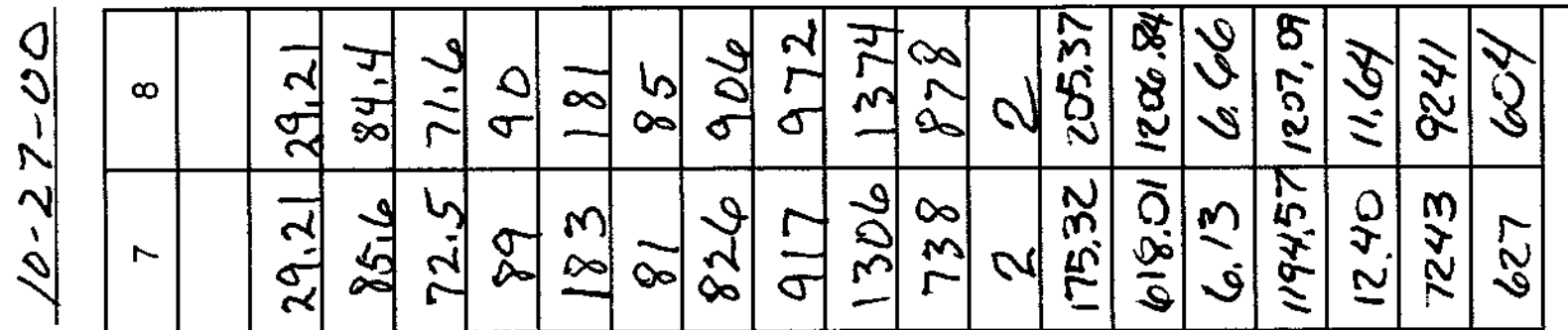

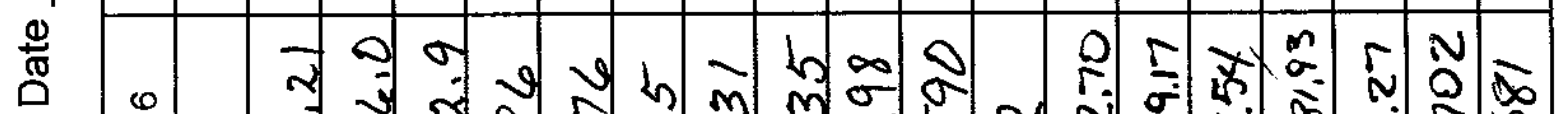

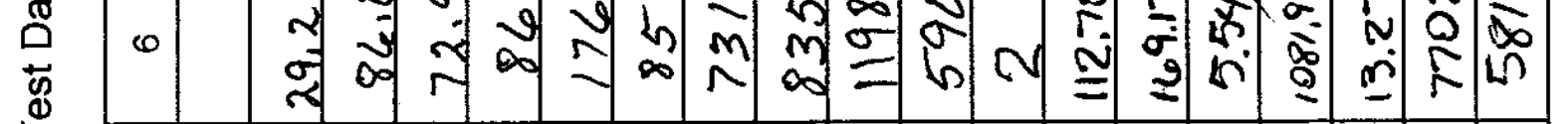

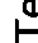

\begin{tabular}{|c|c|c|c|c|c|c|c|c|c|c|c|c|c|c|c|c|c|}
\hline ه & $\begin{array}{l}\text { y } \\
\text { ज्ञ }\end{array}$ & $\begin{array}{l}b \\
\infty\end{array}$ & $\begin{array}{l}0 \\
\\
\end{array}$ & 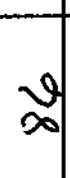 & $\approx$ & $m$ & \begin{tabular}{l}
3 \\
\multirow{3}{*}{}
\end{tabular} & No & 의 & N & & ज. & $\begin{array}{l}\overline{0} \\
\bar{N} \\
=\end{array}$ & $\begin{array}{l}\text { of } \\
\text { vi }\end{array}$ & $\frac{\bar{n}}{\tilde{n}}$ & $\begin{array}{l}0 \\
\tilde{a} \\
\underline{m}\end{array}$ & $\begin{array}{l}\alpha \\
\vdots \\
\vdots \\
0\end{array}$ \\
\hline & ฟิ & $\vec{\infty}$ & m & of & o & $m$ & $\stackrel{m}{n}$ & ఎ & $\tilde{\sigma}$ & $\begin{array}{l}N \\
\infty \\
m\end{array}$ & $N$ & $\frac{2 n}{n}$ & & $\begin{array}{l}m \\
v \\
v\end{array}$ & $\begin{array}{l}5_{0} \\
\overline{0} \\
\infty\end{array}$ & $\begin{array}{l}\tilde{N} \\
\bar{v}\end{array}$ & $\underset{\Sigma}{\stackrel{\Sigma}{*}}$ \\
\hline
\end{tabular}

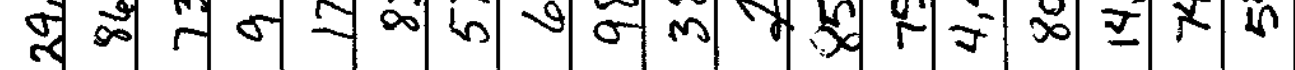

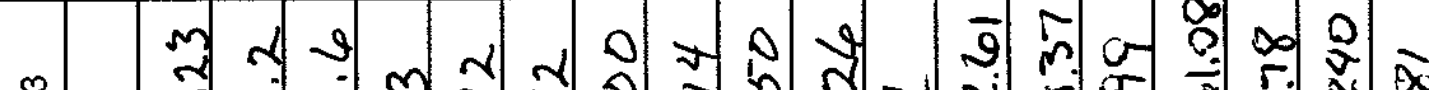

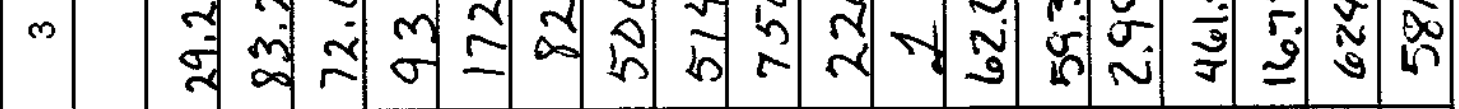

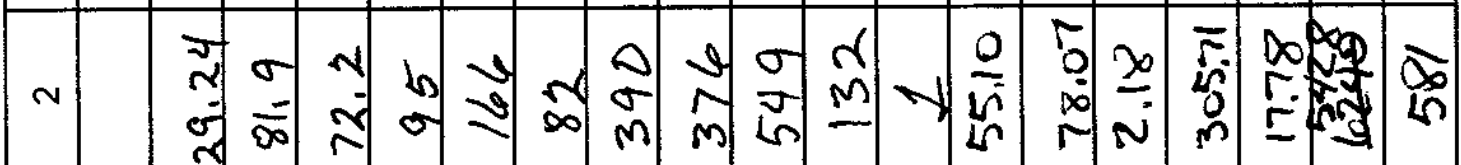

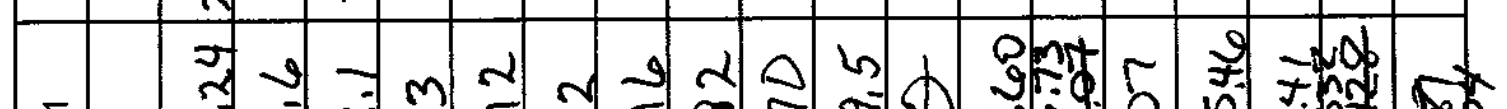

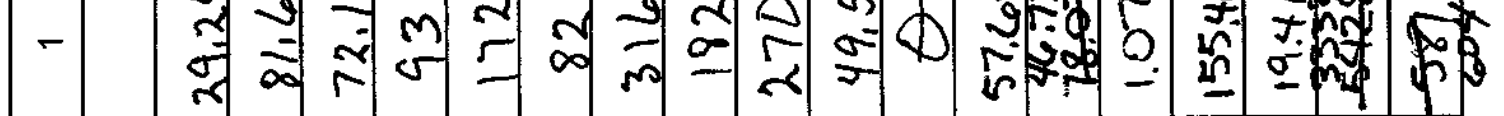

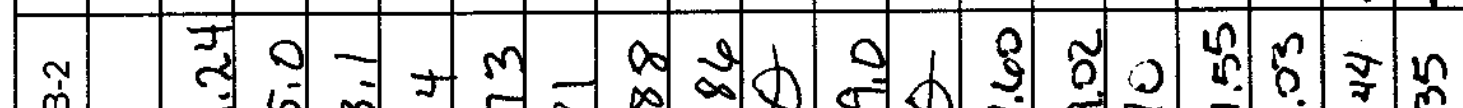

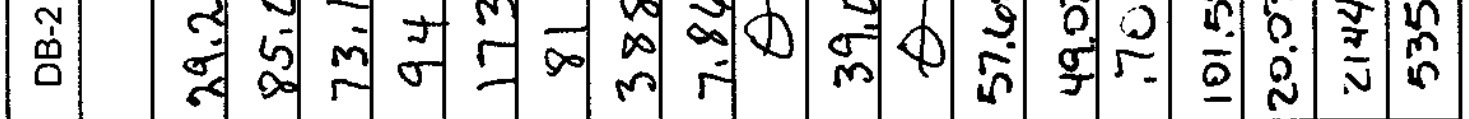

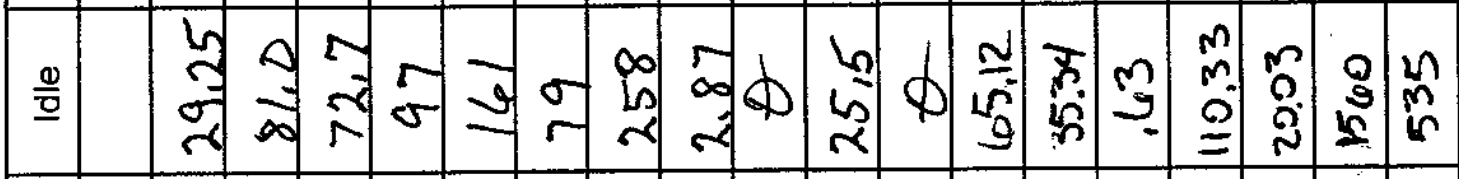

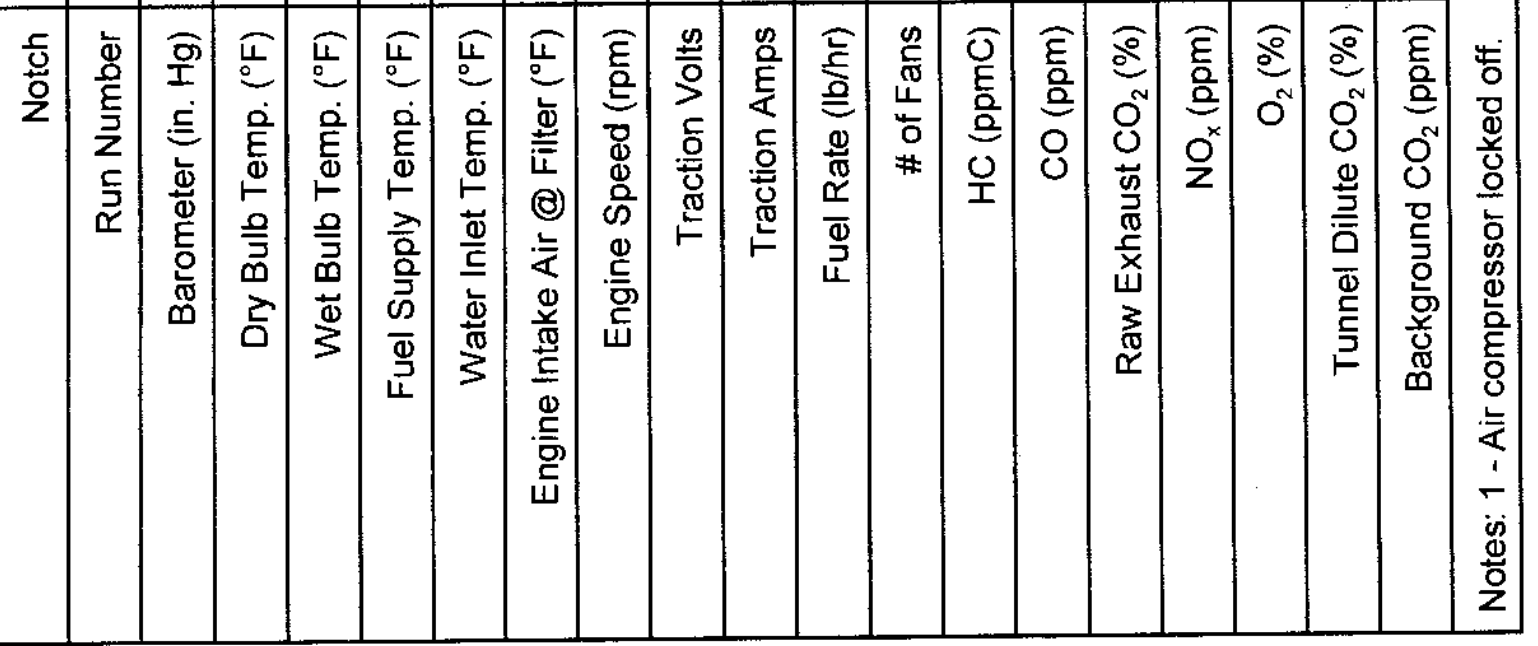

$\xi$

के

1

焉

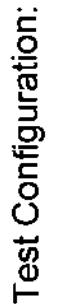




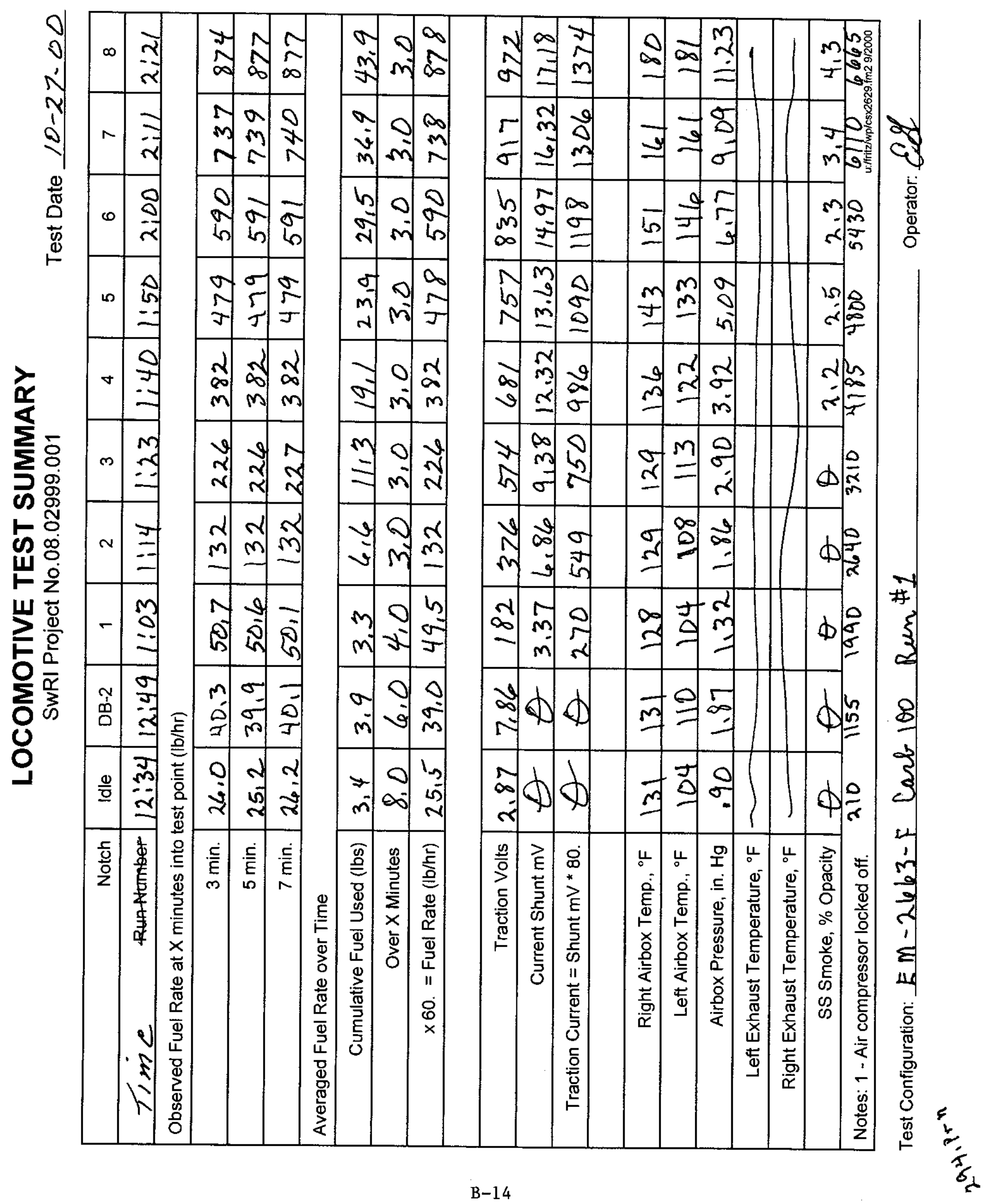




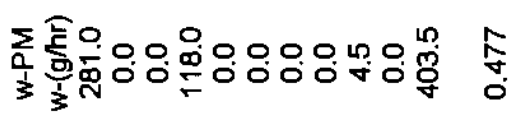

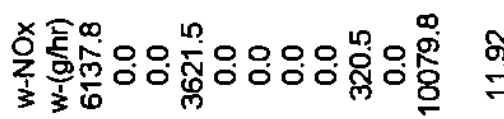

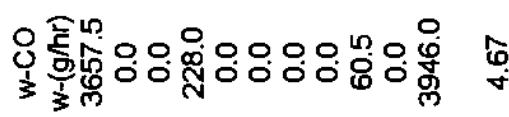

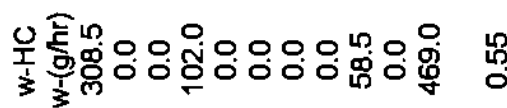

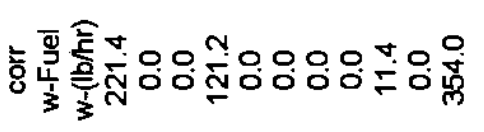

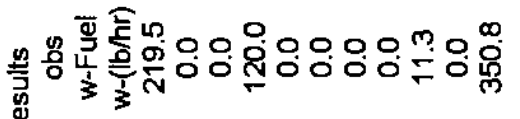

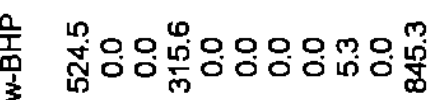

药颉

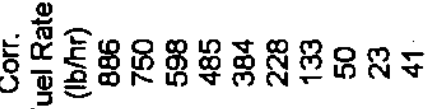

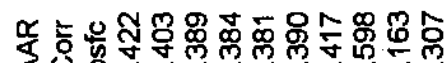

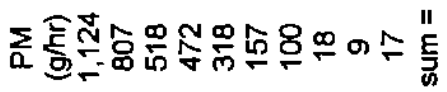

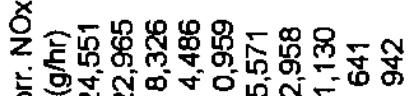

获

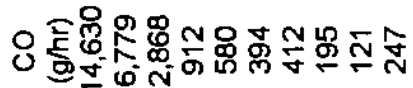

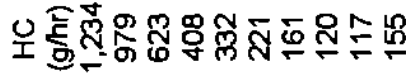

옹

响

$\sum_{i=1}^{\infty}$

罗

要

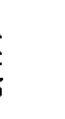

$\sum_{\substack{1 \\ \frac{1}{3}}} \frac{\widehat{E}}{\frac{1}{3}}$

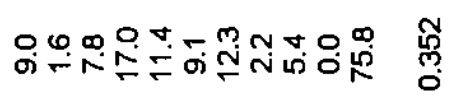

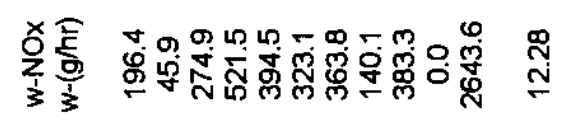

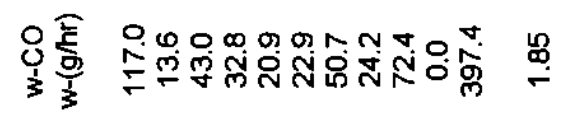

운

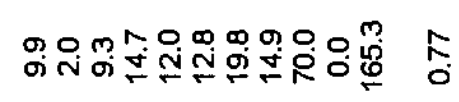

兽

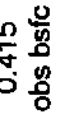

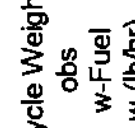

文
这

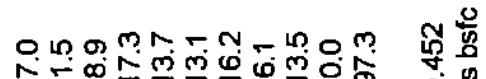

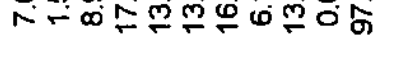

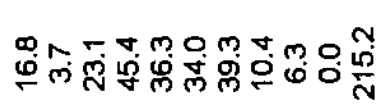

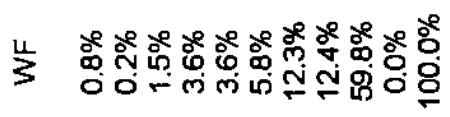
$\circ$ 。

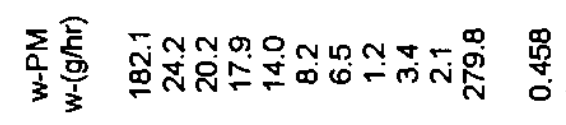

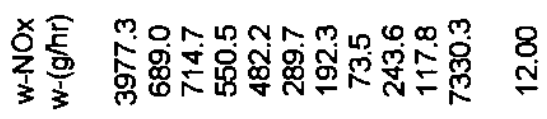

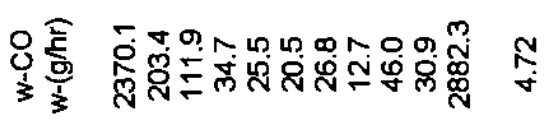

挼

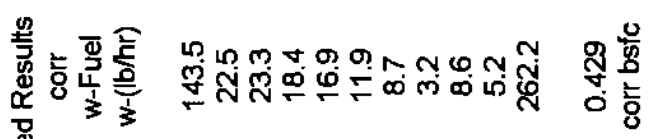

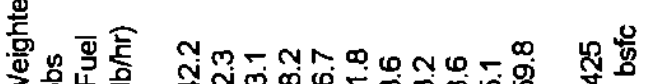

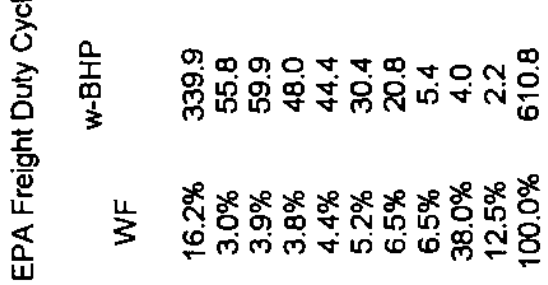




$\begin{array}{ll}\text { LOCOMOTIVE MODEL } & =\text { EMD GP38 } \\ \text { LOCOMOTIVE UNIT \# } & =\text { CSX } \$ 2629 \\ \text { THROTTLE NOTCH } & =\text { IDLE } \\ \text { TEST DATE } & =10 / 27 / 0 \\ \text { TEST NUMBER } & =1 / 10 \\ \text { SWRI FUEL CODE } & =\text { EM-2663-F } \\ \text { BAROMETER } & =29.20(\mathrm{in} . \mathrm{Hg}) \\ \text { DRY BULB TEMP } & =86 .\left({ }^{\circ} \mathrm{F}\right) \\ \text { ABS HUMIDITY } & =97.08(\mathrm{GR} / \mathrm{LB})\end{array}$

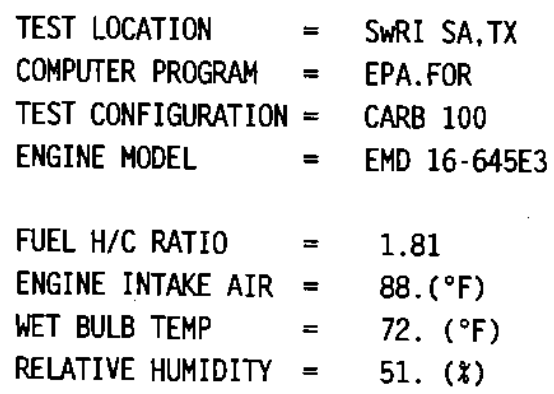

ENGINE PERFORMANCE SUMMARY:

ACCESSORY HORSEPOWER:

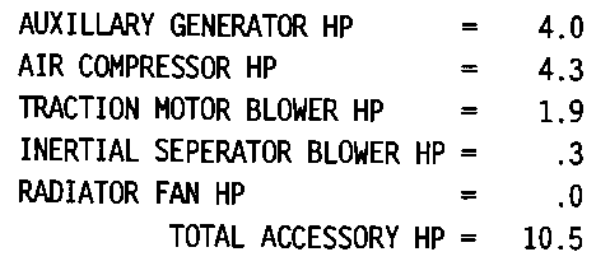

FILTER PAIR I.D. NUMBER

$6756.0 \cdot 41$

.703

38.924

.018

5.054

.091

9.

.856 


$\begin{array}{ll}\text { LOCOMOTIVE MODEL } & =\text { EMD GP38 } \\ \text { LOCOMOTIVE UNIT \# } & =\text { CSX \#2629 } \\ \text { THROTTLE NOTCH } & =\text { DB2 } \\ \text { TEST DATE } & =10 / 27 / 0 \\ \text { TEST NUMBER } & =2 / 10 \\ \text { SWRI FUEL CODE } & =\text { EM-2663-F } \\ \text { BAROMETER } & =29.20(\mathrm{in} . \mathrm{Hg}) \\ \text { DRY BULB TEMP } & =86 .\left(^{\circ} \mathrm{F}\right) \\ \text { ABS HUMIDITY } & =95.74(\mathrm{GR} / \mathrm{LB})\end{array}$

ENGINE PERFORMANCE SUMMARY:

ACCESSORY HORSEPOWER:

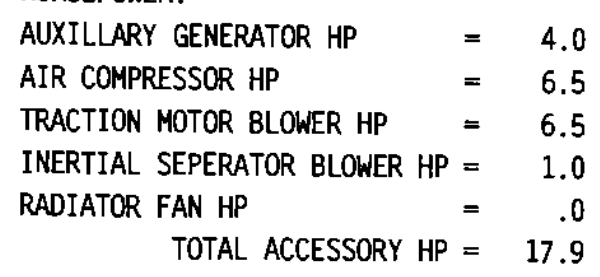

$\begin{array}{rlr}\text { AUXILLARY GENERATOR HP } & =4.0 \\ \text { AIR COMPRESSOR HP } & =6.5 \\ \text { TRACTION MOTOR BLOWER HP } & =6.5 \\ \text { INERTIAL SEPERATOR BLOWER HP } & =1.0 \\ \text { RADIATOR FAN HP } & .0 \\ \text { TOTAL ACCESSORY HP } & =17.9\end{array}$

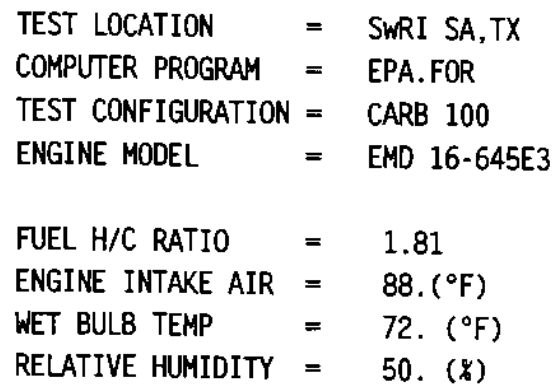

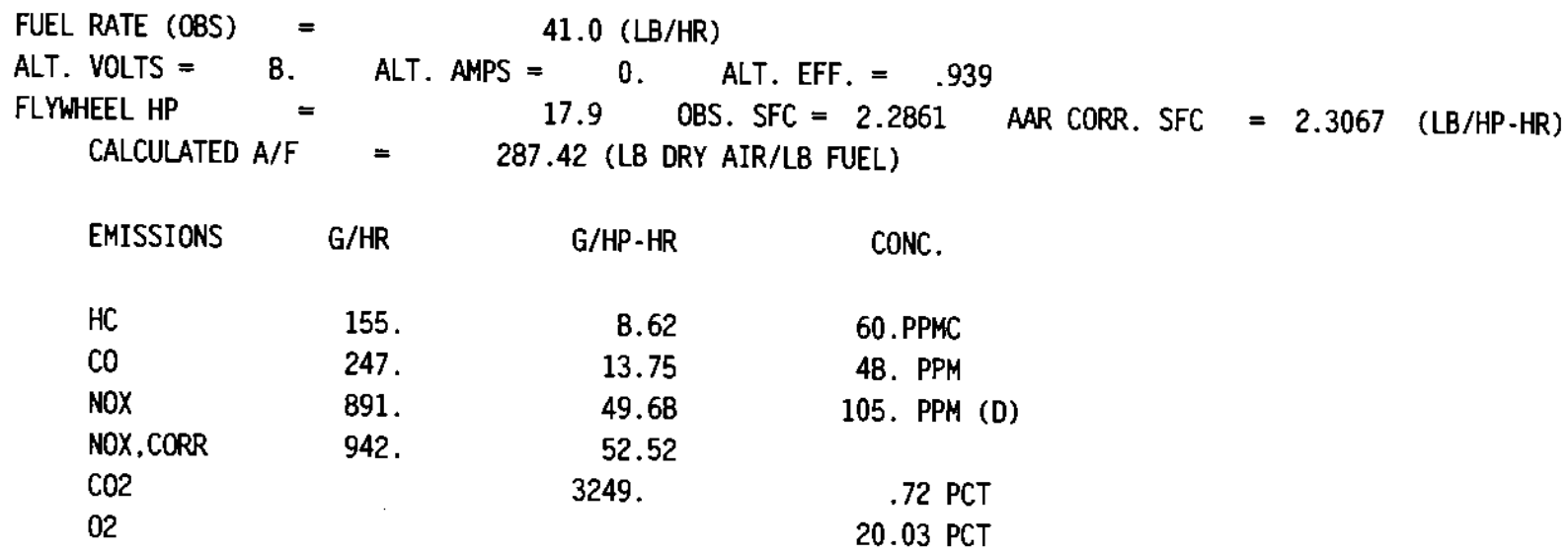

CORRECTION FACTOR SUMMARY:

$\begin{array}{lllll}\text { EPA NOX CF }=1.0573 & \text { NOX-KH } & =1.0469 & \text { NOX-KT } & =1.0000 \\ \text { WET EXH HC CF }=.9890 & \text { AIR TEMP CF }=.9874 & \text { BAROM CF } & =1.0029 \\ \text { FUEL TEMP CF }=.9825 & \text { FUEL S.G. CF }=1.0187 & \text { FUEL HHV CF } & =1.0189\end{array}$

PARTICULATE INFORMATION

FILTER PAIR I.D. NUMBER

FILTER PAIR WEIGHT GAIN (mg)

$6757.0-42$

SAMPLE VOLUME (SCf)

DILUTE PM CONCENTRATION (mg/scf)

38.281

.024

4.502

C02-8ASED DILUTION FACTOR

PARTICULATE CONCENTRATION IN RAW EXHAUST (mg/scf)

.109

PARTICULATE MASS EMISSION RATE $(g / h r)$

17.

BRAKE-SPECIF1C PARTICULATE EMISSION RATE $(\mathrm{g} / \mathrm{hp}-\mathrm{hr})$

.960 


$\begin{array}{ll}\text { LOCOMOTIVE MODEL } & =\text { EMD GP38 } \\ \text { LOCOMOTIVE UNIT \# } & =\text { CSX } \# 2629 \\ \text { THROTTLE NOTCH } & =\text { Notch } 1 \\ \text { TEST DATE } & =10 / 27 / 0 \\ \text { TEST NUMBER } & =3 / 10 \\ \text { SWRI FUEL CODE } & =\text { EM-2663-F } \\ \text { BAROMETER } & =29.19(\mathrm{in} . \mathrm{Hg}) \\ \text { DRY BULB TEMP } & =85 .\left({ }^{\circ} \mathrm{F}\right) \\ \text { ABS HUMIDITY } & =94.86(\mathrm{GR} / \mathrm{LB})\end{array}$

$\begin{array}{lll}\text { TEST LOCATION } & = & \text { SWRI SA,TX } \\ \text { COMPUTER PROGRAM } & =\text { EPA. FOR } \\ \text { TEST CONFIGURATION } & =\text { CARB } 100 \\ \text { ENGINE MODEL } & =\text { EMD } 16 \cdot 645 E 3 \\ \text { FUEL H/C RATIO } & =1.81 \\ \text { ENGINE INTAKE AIR } & =87 .\left(^{\circ} \mathrm{F}\right) \\ \text { WET BULB TEMP } & =71 .\left({ }^{\circ} \mathrm{F}\right) \\ \text { RELAT IVE HUMIDITY } & =52 .(\%)\end{array}$

ENGINE PERFORMANCE SUMMARY:

ACCESSORY HORSEPOWER:

$\begin{array}{rrr}\text { AUXILLARY GENERATOR HP } & =4.0 \\ \text { AIR COMPRESSOR HP } & =5.3 \\ \text { TRACTION MOTOR BLOWER HP } & =3.5 \\ \text { INERTIAL SEPERATOR BLOWER HP } & =.5 \\ \text { RADIATOR FAN HP } & =.0 \\ \text { TOTAL ACCESSORY HP } & =13.4\end{array}$

FUEL RATE (OBS) =

$49.5(\mathrm{LB} / \mathrm{HR})$
ALT. AMPS $=270 . \quad$ ALT. EFF. $=.939$

ALT. VOLTS $=182$.

FLYWHEEL HP

B3.6 OBS. SFC $=.5923$ AAR CORR. SFC $=.5979(\mathrm{LB} / \mathrm{HP}-\mathrm{HR})$

CALCULATED A/F 192.94 (LB DRY AIR/LB FUEL)

EMISSIONS G/HR G/HP-HR CONC.

$\begin{array}{lrrr}\text { HC } & 120 . & 1.44 & \text { 58.PPMC } \\ \text { CO } & 195 . & 2.33 & 47 . \text { PPM } \\ \text { NOX } & 1073 . & 12.84 & 157 . \text { PPM (D) } \\ \text { NOX.CORR } & 1130 . & 13.52 & \\ \text { CO2 } & & 846 . & 1.08 \text { PCT } \\ 02 & & & 19.53 \text { PCT }\end{array}$

CORRECTION FACTOR SUMMARY:

EPA NOX CF $=1.0531$

WET EXH HC CF $=.9858$

FUEL TEMP CF $=.9860$

$$
\begin{array}{ll}
\text { NOX-KH } & =1.0434 \\
\text { AIR TEMP CF } & =.9878
\end{array}
$$$$
\text { FUEL S.G. CF }=1.0187
$$

PARTICULATE INFORMATION

$\begin{array}{ll}\text { NOX-KT } & =1.0000 \\ \text { BAROM CF } & =1.0029 \\ \text { FUEL HHV CF } & =1.0189\end{array}$

FILTER PAIR I.D. NUMBER

FILTER PAIR WEIGHT GAIN (mg)

SAMPLE VOLUME (sCf)

DILUTE PM CONCENTRATION (mg/scf)

CO2-BASED DILUTION FACTOR

PARTICULATE CONCENTRATION IN RAW EXHAUST (mg/scf)

PARTICULATE MASS EMISSION RATE $(g / h r)$

BRAKE-SPECIFIC PARTICULATE EMISSION RATE $(g / h p-h r)$
$6758.0-43$

1.516

38.093

.040

3.581

.143

18.

.218 


\begin{tabular}{|c|c|c|c|}
\hline LOCOMOTIVE MODEL & EMD GP38 & TEST LOCATION & SWRI SA,TX \\
\hline LOCOMOTIVE UNIT \# & $\operatorname{cs} x \# 2629$ & COMPUTER PROGRAM & EPA.FOR \\
\hline THROTTLE NOTCH & Notch 2 & TEST CONFIGURATION & CARB 100 \\
\hline TEST DATE & $10 / 27 / 0$ & ENGINE MODEL & EMD 16-645E3 \\
\hline TEST NUMBER & $4 / 10$ & & \\
\hline SWRI FUEL CODE & EM-2663-F & FUEL H/C RATIO & 1.81 \\
\hline BAROMETER & $29.19($ in. $\mathrm{Hg})$ & ENGINE INTAKE AIR & $86 .\left({ }^{\circ} \mathrm{F}\right)$ \\
\hline DRY BULB TEMP & 85. $\left({ }^{\circ} \mathrm{F}\right)$ & WET BULB TEMP & 71. $\left({ }^{\circ} \mathrm{F}\right)$ \\
\hline ABS HUMIDITY & $94.69(G R / L B)$ & RELATIVE HUMIDITY & 51. $(\%)$ \\
\hline
\end{tabular}

ENGINE PERFORMANCE SUMMARY:

ACCESSORY HORSEPOWER:

$\begin{array}{rlr}\text { AUXILLARY GENERATOR HP } & =4.0 \\ \text { AIR COMPRESSOR HP } & =6.5 \\ \text { TRACTION MOTOR BLOWER HP } & =6.6 \\ \text { INERTIAL SEPERATOR BLOWER HP } & =1.0 \\ \text { RADIATOR FAN HP } & 3.1 \\ \text { TOTAL ACCESSORY HP } & =21.2\end{array}$

FUEL RATE (OBS) =

132.0 (LB/HR)

ALT. VOLTS $=377$.

ALT. AMPS $=554 . \quad$ ALT. EFF. $=.939$

FLYWHEEL HP $=\quad 319.5$ OBS. SFC = .4131 AAR CORR. SFC $=\quad .4172$ (LB/HP-HR)

CALCULATED A/F $\quad=\quad 96.40$ (LB DRY AIR/LB FUEL)

EMISSIONS G/HR G/HP-HR CONC.

HC $\quad 161.50 \quad 58 . P P M C$

CO 412 . 1.29 75. PPH

NOX 2820. $8.83 \quad 311$. PPH (D)

NOX, CORR 2958. $\quad 9.26$

CO2 592 . 2.18 PCT

$02 \quad 17.91$ PCT

CORRECTION FACTOR SUMMARY:

$\begin{array}{lllll}\text { EPA NOX CF }=1.0488 & \text { NOX-KH } & =1.0399 & \text { NOX-KT } & =1.0000 \\ \text { WET EXH HC CF }=.9762 & \text { AIR TEMP CF }=.9883 & \text { BAROH CF } & =1.0029 \\ \text { FUEL TEMP CF }=.9850 & \text { FUEL S.G. CF }=1.0187 & \text { FUEL HHV CF } & =1.0189\end{array}$

PARTICULATE INFORMATION

.......................

FILTER PAIR I.D. NUMBER

$$
\begin{array}{r}
6759.0-44 \\
5.049 \\
38.274 \\
.132 \\
4.410 \\
.582 \\
100 .
\end{array}
$$

.312 


$\begin{array}{ll}\text { LOCOMOTIVE MODEL } & =\text { EMD GP38 } \\ \text { LOCOMOTIVE UNIT \# } & =\text { CSX } \# 2629 \\ \text { THROTTLE NOTCH } & =\text { Notch } 3 \\ \text { TEST DATE } & =10 / 27 / 0 \\ \text { TEST NUMBER } & =5 / 10 \\ \text { SWRI FUEL CODE } & =\text { EM-2663-F } \\ \text { BAROMETER } & =29.19(\mathrm{in} . \mathrm{Hg}) \\ \text { DRY BULB TEMP } & =85 .\left({ }^{\circ} \mathrm{F}\right) \\ \text { ABS HUMIDITY } & =96.49(\mathrm{GR} / \mathrm{LB})\end{array}$

ENGINE PERFORMANCE SUMMARY:

ACCESSORY HORSEPOWER:

$\begin{array}{rlr}\text { AUXILLARY GENERATOR HP } & = & 4.0 \\ \text { AIR COMPRESSOR HP } & = & 8.3 \\ \text { TRACTION MOTOR BLOWER HP } & = & 13.9 \\ \text { INERTIAL SEPERATOR BLOWER HP } & = & 2.1 \\ \text { RADIATOR FAN HP } & = \\ \text { TOTAL ACCESSORY HP } & =34.8\end{array}$

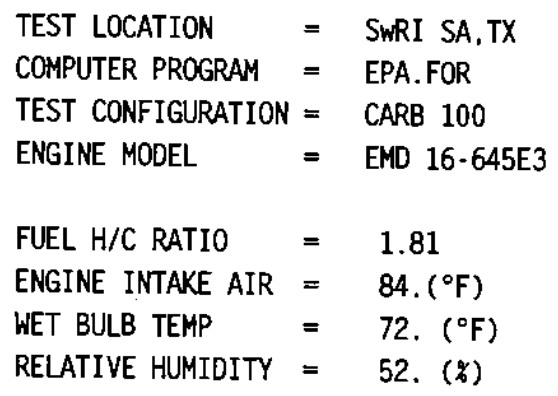




$\begin{array}{llll}\text { LOCOMOTIVE MODEL } & =\text { EMD GP38 } & \text { TEST LOCATION } & =\text { SWRI SA,TX } \\ \text { LOCOMOTIVE UNIT \# } & =\text { CSX \#2629 } & \text { COMPUTER PROGRAM } & =\text { EPA.FOR } \\ \text { THROTTLE NOTCH } & =\text { Notch } 4 & \text { TEST CONFIGURATION } & \text { CARB } 100 \\ \text { TEST DATE } & =10 / 27 / 0 & \text { ENGINE MODEL } & =\text { EMD } 16.645 E 3 \\ \text { TEST NUMBER } & =6 / 10 & & \\ \text { SWRI FUEL CODE } & =\text { EM-2663-F } & \text { FUEL H/C RATIO } & =1.81 \\ \text { BAROMETER } & =29.19(\mathrm{in} . \mathrm{Hg}) & \text { ENGINE INTAKE AIR } & =86 .\left(^{\circ} \mathrm{F}\right) \\ \text { DRY BULB TEMP } & =85 .\left({ }^{\circ} \mathrm{F}\right) & \text { WET BULB TEMP } & =71 .\left({ }^{\circ} \mathrm{F}\right) \\ \text { ABS HUMIDITY } & =95.57(\mathrm{GR} / \mathrm{LB}) & \text { RELATIVE HUMIDITY } & =51 .(\%)\end{array}$

ENGINE PERFORMANCE SUMMARY:

ACCESSORY HORSEPOWER:

$\begin{array}{llr}\text { AUXILLARY GENERATOR HP } & = & 4.0 \\ \text { AIR COMPRESSOR HP } & = & 9.6 \\ \text { TRACTION MOTOR BLOWER HP } & = & 21.1 \\ \text { INERTIAL SEPERATOR BLOWER HP } & = & 3.2 \\ \text { RADIATOR FAN HP } & = & 9.7\end{array}$

TOTAL ACCESSORY HP $=47.5$

FUEL RATE (OBS) $=380.0(\mathrm{LB} / \mathrm{HR})$

ALT. VOLTS $=682 . \quad$ ALT. AMPS $=9 B 6 . \quad$ ALT. EFF. $=.939$

FLYHEEL HP $=100 \mathrm{~B} .2$ OBS. SFC $=.3769$ AAR CORR. SFC $=.3806(\mathrm{LB} / \mathrm{HP}-\mathrm{HR})$

CALCULATED A/F $=47.62$ (LB DRY AIR/LB FUEL)

$\begin{array}{llll}\text { EMISSIONS } & \text { G/HR } & \text { G/HP.HR } & \text { CONC. }\end{array}$

$\begin{array}{lrrc}\text { HC } & 332 . & .33 & 83 . \text { PPMC } \\ \text { CO } & 5 B 0 . & .57 & 75 . \text { PPM } \\ \text { NOX } & 10435 . & 10.35 & \text { B17. PPM (D) } \\ \text { NOX.CORR } & 10959 . & 10.87 & \\ \text { C02 } & & 542 . & 4.47 \text { PCT } \\ 02 & & & 14.65 \text { PCT }\end{array}$

CORRECTION FACTOR SUMMARY:

$\begin{array}{lllll}\text { EPA NOX CF }=1.0502 & \text { NOX-KH } & =1.0411 & \text { NOX-KT } & =1.0000 \\ \text { WET EXH HC CF }=.9568 & \text { AIR TEMP CF }=.9883 & \text { BAROM CF } & =1.0029 \\ \text { FUEL TEMP CF }=.9860 & \text { FUEL S.G. CF }=1.0187 & \text { FUEL HHV CF } & =1.0189\end{array}$

PARTICULATE INFORMATION

FILTER PAIR I.D. NUMBER

$6761.0-46$

7.801

37.512

SAMPLE VOLUME (SCf)

.208

6.216

1.293

PARTICULATE CONCENTRATION IN RAW EXHAUST (mg/scf)

318.

BRAKE-SPECIFIC PARTICULATE EMISSION RATE $(\mathrm{g} / \mathrm{hp}-\mathrm{hr})$

.315 


$\begin{array}{ll}\text { LOCOMOTIVE MODEL } & =\text { EMD GP38 } \\ \text { LOCOMOTIVE UNIT \# } & =\text { CSX \#2629 } \\ \text { THROTTLE NOTCH } & =\text { Notch } 5 \\ \text { TEST DATE } & =10 / 27 / 0 \\ \text { TEST NUMBER } & =7 / 10 \\ \text { SWRI FUEL CODE } & =\text { EM-2663-F } \\ \text { BAROMETER } & =29.18(\text { in. Hg) } \\ \text { DRY BULB TEMP } & =86 .\left({ }^{\circ} \mathrm{F}\right) \\ \text { ABS HUMIDITY } & =98.00(\mathrm{GR} / \mathrm{LB})\end{array}$

ENGINE PERFORMANCE SUMMARY:

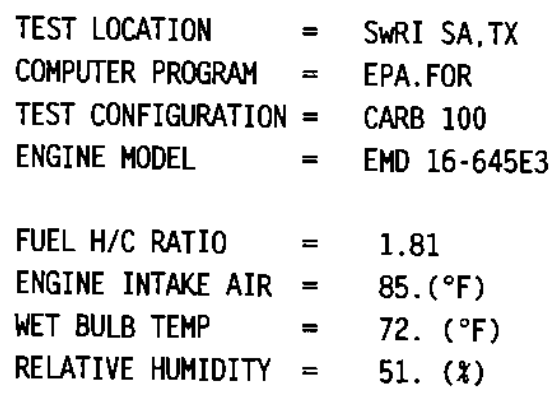

ACCESSORY HORSEPOWER:

$\begin{array}{rrr}\text { AUXILLARY GENERATOR HP } & = & 4.0 \\ \text { AIR COMPRESSOR HP } & =10.8 \\ \text { TRACTION MOTOR BLOWER HP } & =30.1 \\ \text { INERTIAL SEPERATOR BLOWER HP } & =4.5 \\ \text { RADIATOR FAN HP } & 27.8 \\ \text { TOTAL ACCESSORY HP } & =77.2\end{array}$

FUEL RATE (OBS) =

ALT. VOLTS $=759$.

480.0 (LB/HR)

$\begin{array}{lccc}\text { FLYWHEEL HP } & & 1262.3 & \text { OBS. SFC }= \\ \text { CALCULATED A/F } & = & 42.40 & \text { (LB DRY AIR/LB FUEL) } \\ \text { EMISSIONS } & \text { G/HR } & \text { G/HP-HR } & \text { CONC. } \\ & & & \\ \text { HC } & 408 . & .32 & 90 . \text { PPMC } \\ \text { CO } & 912 . & .72 & 105 . \text { PPM } \\ \text { NOX } & 13715 . & 10.86 & 957 . \text { PPM (D) } \\ \text { NOX,CORR } & 14486 . & 11.48 & \\ \text { C02 } & & 546 . & 5.03 \text { PCT } \\ \text { O2 } & & & 14.02 \text { PCT }\end{array}$

CORRECTION FACTOR SUMMARY:

$\begin{array}{lllll}\text { EPA NOX CF }=1.0562 & \text { NOX-KH } & =1.0460 & \text { NOX-KT } & =1.0000 \\ \text { WET EXH HC CF }=.9520 & \text { AIR TEMP CF }=.9887 & \text { BAROM CF } & =1.0028 \\ \text { FUEL TEMP CF }=.9855 & \text { FUEL S.G. CF }=1.0187 & \text { FUEL HHV CF } & =1.0189\end{array}$

PARTICULATE INFORMATION

FILTER PAIR I.D. NUMBER

$6762.0 \cdot 47$

FILTER PAIR WEIGHT GAIN (mg)

37.329

DILUTE PM CONCENTRATION (mg/scf)

9.127

1.702

PARTICULATE CONCENTRATION IN RAW EXHAUST (mg/scf)

PARTICULATE MASS EMISSION RATE $(\mathrm{g} / \mathrm{hr})$

472 .

BRAKE-SPECIFIC PARTICULATE EMISSION RATE $(\mathrm{g} / \mathrm{hp}-\mathrm{hr})$ 


$\begin{array}{ll}\text { LOCOMOTIVE MODEL } & =\text { EMD GP38 } \\ \text { LOCOMOTIVE UNIT \# } & =\text { CSX \#2629 } \\ \text { THROTTLE NOTCH } & =\text { Notch } 6 \\ \text { TEST DATE } & =10 / 27 / 0 \\ \text { TEST NUMBER } & =8 / 10 \\ \text { SWRI FUEL CODE } & =\text { EM-2663-F } \\ \text { BAROMETER } & =29.18(\mathrm{in} . \mathrm{Hg}) \\ \text { DRY BULB TEMP } & =86 .\left({ }^{\circ} \mathrm{F}\right) \\ \text { ABS HUMIDITY } & =94.31(\mathrm{GR} / \mathrm{LB})\end{array}$

ENGINE PERFORMANCE SUMMARY:

ACCESSORY HORSEPOWER:

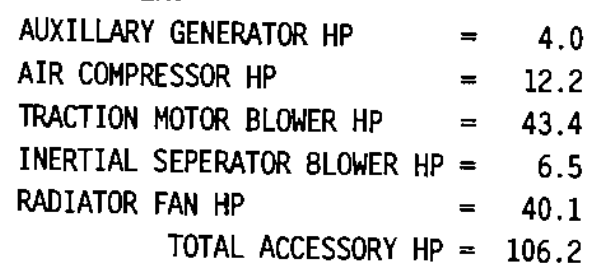

$\begin{array}{rlr}\text { AUXILLARY GENERATOR HP } & = & 4.0 \\ \text { AIR COMPRESSOR HP } & = & 12.2 \\ \text { TRACTION MOTOR BLOWER HP } & = & 43.4 \\ \text { INERTIAL SEPERATOR 8LOWER HP } & = & 6.5 \\ \text { RADIATOR FAN HP } & = & 40.1 \\ \text { TOTAL ACCESSORY HP } & = & 106.2\end{array}$

$\begin{array}{ll}\text { TEST LOCATION } & =\text { SWRI SA, TX } \\ \text { COMPUTER PROGRAM } & =\text { EPA.FOR } \\ \text { TEST CONFIGURATION } & =\text { CARB } 100 \\ \text { ENGINE MODEL } & =\text { EMD } 16-645 E 3 \\ \text { FUEL H/C RATIO } & =1.81 \\ \text { ENGINE INTAKE AIR } & =86 .\left({ }^{\circ} \mathrm{F}\right) \\ \text { WET BULB TEMP } & =72 .\left({ }^{\circ} \mathrm{F}\right) \\ \text { RELATIVE HUMIDITY } & =49 .(\%)\end{array}$

CORRECTION FACTOR SUMMARY:

$\begin{array}{lllll}\text { EPA NOX CF }=1.0470 & \text { NOX-KH } & =1.0385 & \text { NOX-KT } & =1.0000 \\ \text { WET EXH HC CF }=.9479 & \text { AIR TEMP CF }=.9883 & \text { BAROM CF } & =1.0028 \\ \text { FUEL TEMP CF }=.9865 & \text { FUEL S.G. CF }=1.0187 & \text { FUEL HHV CF } & =1.0189\end{array}$

PARTICULATE INFORMATION

FILTER PAIR I.D. NUMBER

FILTER PAIR WEIGHT GAIN (mg)

$6763.0-48$

8.850

36.968

SAMPLE VOLUME (scf)

DILUTE PM CONCENTRATION (mg/scf)

.239

CO2-BASED DILUTION FACTOR

6.962

1.667

PARTICULATE MASS EMISSION RATE $(\mathrm{g} / \mathrm{hr})$

518.

BRAKE-SPECIFIC PARTICULATE EMISSION RATE $(\mathrm{g} / \mathrm{hp} \cdot \mathrm{hr})$ 


$\begin{array}{ll}\text { LOCOMOTIVE MODEL } & =\text { EMD GP38 } \\ \text { LOCOMOTIVE UNIT \# } & =\text { CSX \#2629 } \\ \text { THROTTLE NOTCH } & =\text { Notch } 7 \\ \text { TEST DATE } & =10 / 27 / 0 \\ \text { TEST NUM8ER } & =9 / 10 \\ \text { SWRI FUEL CODE } & =\text { EM-2663-F } \\ \text { BAROMETER } & =29.18(\mathrm{in} . \mathrm{Hg}) \\ \text { DRY BULB TEMP } & =86 .\left({ }^{\circ} \mathrm{F}\right) \\ \text { ABS HUMIDITY } & =93.50(\mathrm{GR} / \mathrm{LB})\end{array}$

$\begin{array}{lll}\text { TEST LOCATION } & =\text { SWRI SA,TX } \\ \text { COMPUTER PROGRAM } & =\text { EPA.FOR } \\ \text { TEST CONFIGURATION } & =\text { CARB } 100 \\ \text { ENGINE MODEL } & =\text { EMD } 16-645 E 3 \\ \text { FUEL H/C RATIO } & =1.81 \\ \text { ENGINE INTAKE AIR } & =85 .\left(^{\circ} \mathrm{F}\right) \\ \text { WET BULB TEMP } & =71 .\left(^{\circ} \mathrm{F}\right) \\ \text { RELATIVE HUMIDITY } & =49 .(\%)\end{array}$

ENGINE PERFORMANCE SUMMARY:

ACCESSORY HORSEPOWER:

$\begin{array}{rrr}\text { AUXILLARY GENERATOR HP } & =4.0 \\ \text { AIR COMPRESSOR HP } & =13.8 \\ \text { TRACTION MOTOR BLOWER HP } & 62.6 \\ \text { INERTIAL SEPERATOR BLOWER HP } & =9.4 \\ \text { RADIATOR FAN HP } & 57.9 \\ \text { TOTAL ACCESSORY HP } & =147.7\end{array}$

FUEL RATE (OBS) = 742.0 (LB/HR)

ALT. VOLTS $=917 . \quad$ ALT. AMPS $=1306 . \quad$ ALT. EFF. $=.939$

FLYWHEEL HP $=\quad 1858.5 \quad$ OBS. SFC $=.3992 \quad$ AAR CORR. SFC $=.4033$ (LB/HP-HR)

CALCULATED A/F $=34.64$ (LB DRY AIR/L8 FUEL)

EMISSIONS G/HR G/HP.HR CONC.

$\begin{array}{lrrr}\text { HC } & 979 . & .53 & 170 . \text { PPMC } \\ \text { CO } & 6779 . & 3.65 & 618 . \text { PPM } \\ \text { NOX } & 21977 . & 11.82 & 1220 . \text { PPM (D) } \\ \text { NOX, CORR } & 22965 . & 12.36 & \\ \text { CO2 } & & 569 . & 6.13 \text { PCT } \\ 02 & & & 12.40 \text { PCT }\end{array}$

CORRECTION FACTOR SUMMARY:

EPA NOX CF $=1.0450$

WET EXH HC CF $=.9430$

NOX-KH $\quad=1.0368$

NOX-KT $\quad=1.0000$

FUEL TEMP CF $=.9835$

AIR TEMP CF $=.9887$

BAROM CF $\quad=1.0028$

FUEL S.G. CF $=1.0187 \quad$ FUEL HHV CF $=1.0189$

PARTICULATE INFORMATION

FILTER PAIR I.D. NUMBER

FILTER PAIR WEIGHT GAIN (mg)

$6764.0-49$

SAMPLE VOLUME (SCf)

DILUTE PM CONCENTRATION (mg/scf)

7.349

36.910

C02-8ASED DILUTION FACTOR

.199

11.520

2.294

PARTICULATE CONCENTRATION IN RAW EXHAUST (mg/scf)

PARTICULATE MASS EMISSION RATE $(\mathrm{g} / \mathrm{hr})$

807.

BRAKE-SPECIFIC PARTICULATE EMISSION RATE $(g / h p-h r)$ 


$\begin{array}{ll}\text { LOCOMOTIVE MODEL } & =\text { EMD GP38 } \\ \text { LOCOMOTIVE UNIT \# } & =\text { CSX } \# 2629 \\ \text { THROTTLE NOTCH } & =\text { Notch } 8 \\ \text { TEST DATE } & =10 / 27 / 0 \\ \text { TEST NUMBER } & =10 / 10 \\ \text { SWRI FUEL CODE } & =\text { EM-2663-F } \\ \text { BAROMETER } & =29.18(\mathrm{in} . \mathrm{Hg}) \\ \text { DRY BULB TEMP } & =86 .\left(^{\circ} \mathrm{F}\right) \\ \text { ABS HUMIDITY } & =93.60(\mathrm{GR} / \mathrm{LB})\end{array}$

ENGINE PERFORMANCE SUMMARY:

ACCESSORY HORSEPOWER:

$\begin{array}{rrr}\text { AUXILLARY GENERATOR HP } & =4.0 \\ \text { AIR COMPRESSOR HP } & 15.1 \\ \text { TRACTION MOTOR BLOWER HP } & =82.7 \\ \text { INERTIAL SEPERATOR BLOWER HP } & =12.4 \\ \text { RADIATOR FAN HP } & 76.5 \\ \text { TOTAL ACCESSORY HP } & =190.7\end{array}$

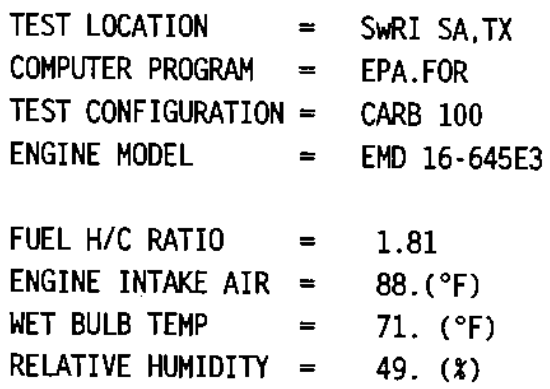

878.0 (LB/HR)

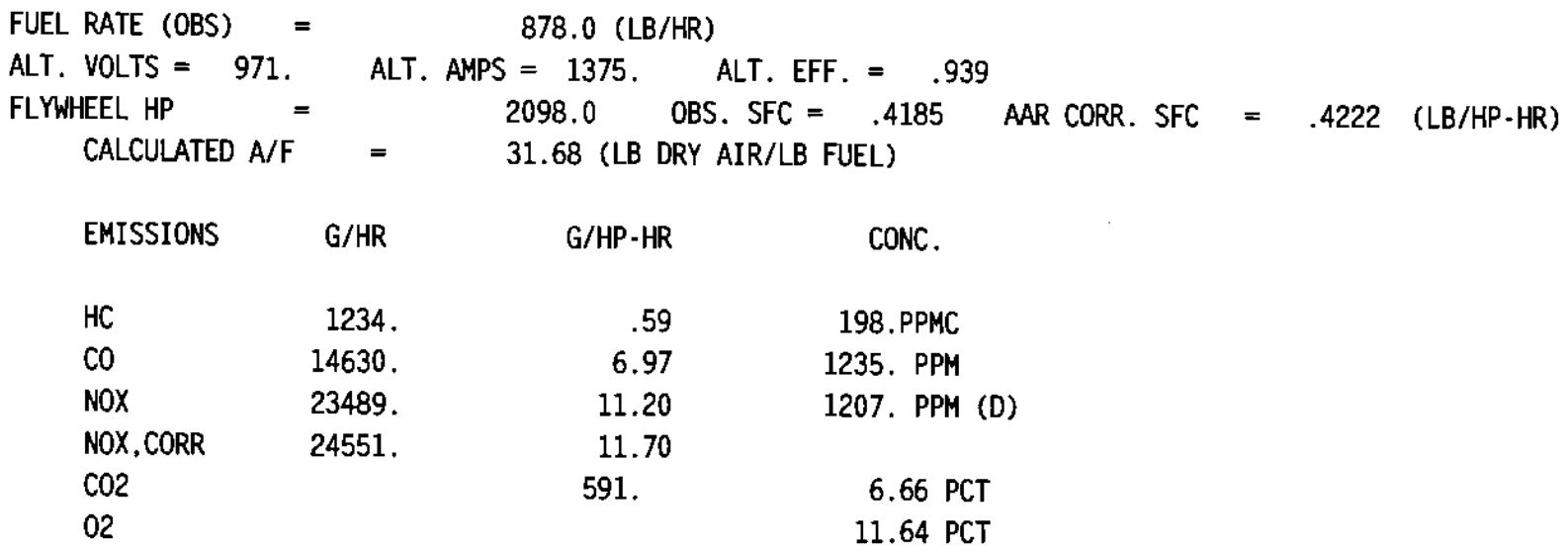

CORRECTION FACTOR SUMMARY:

$\begin{array}{lllll}\text { EPA NOX CF }=1.0452 & \text { NOX-KH } & =1.0370 & \text { NOX-KT } & =1.0000 \\ \text { WET EXH HC CF= } & .9384 & \text { AIR TEMP CF }=.9874 & \text { BAROM CF } & =1.0028 \\ \text { FUEL TEMP CF }=.9845 & \text { FUEL S.G. CF }=1.0187 & \text { FUEL HHV CF } & =1.0189\end{array}$

PARTICULATE INFORMATION

FILTER PAIR I.D. NUMBER

$6765.0-50$

FILTER PAIR WEIGHT GAIN (mg)

15.194

35.353

SAMPLE VOLUME (sCf)

DILUTE PM CONCENTRATION (mg/scf)

6.848

PARTICULATE CONCENTRATION IN RAW EXHAUST ( $\mathrm{mg} / \mathrm{scf}$ )

2.943

PARTICULATE MASS EMISSION RATE $(\mathrm{g} / \mathrm{hr})$

1124 .

BRAKE-SPECIFIC PARTICULATE EMISSION RATE $(g / h p-h r)$ 


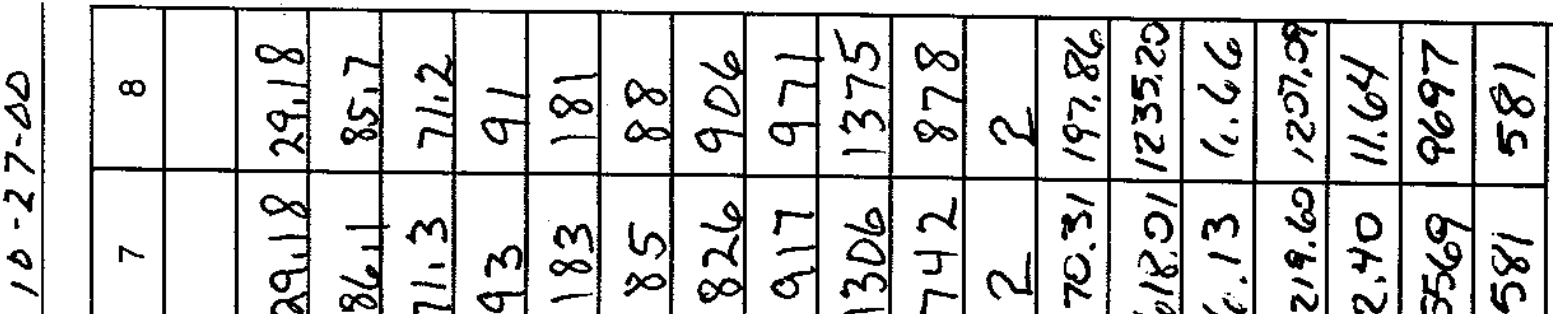
.

要

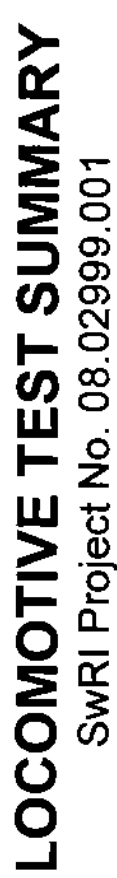

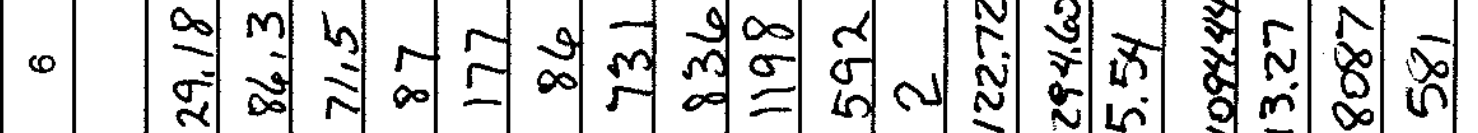

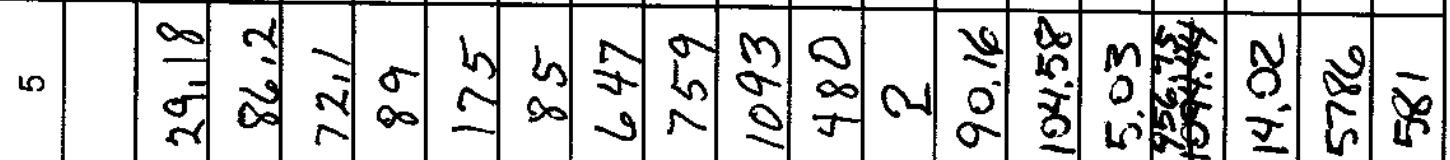

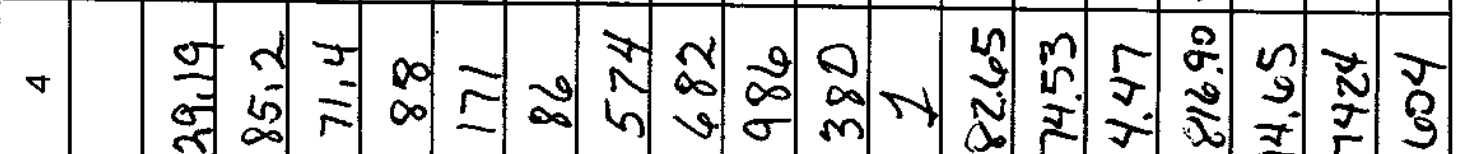

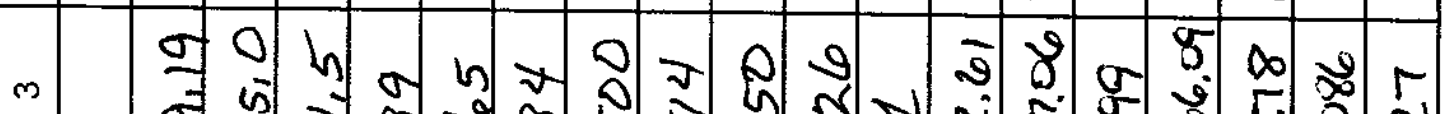

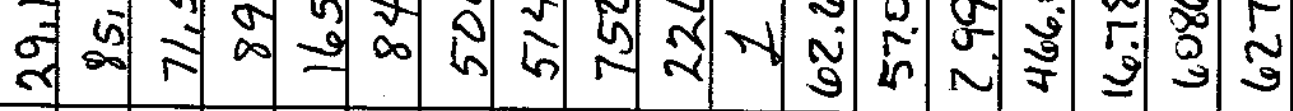

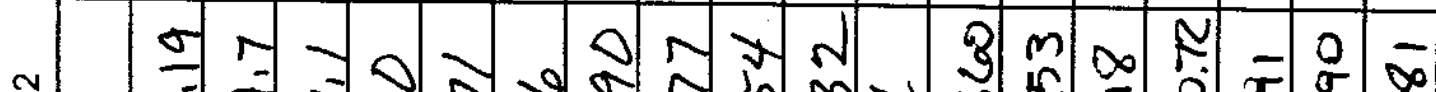

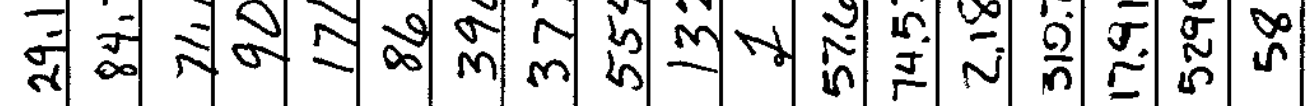

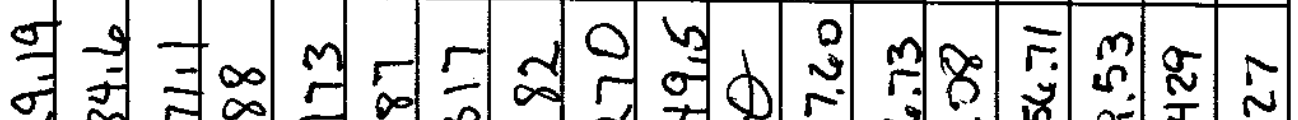

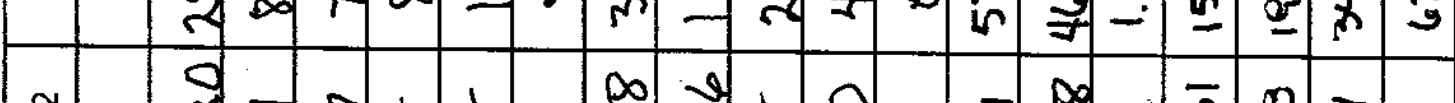

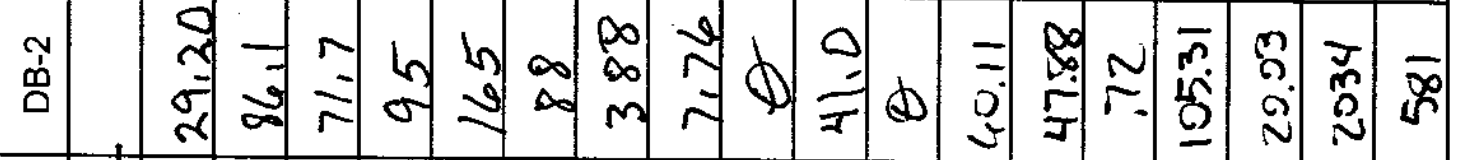

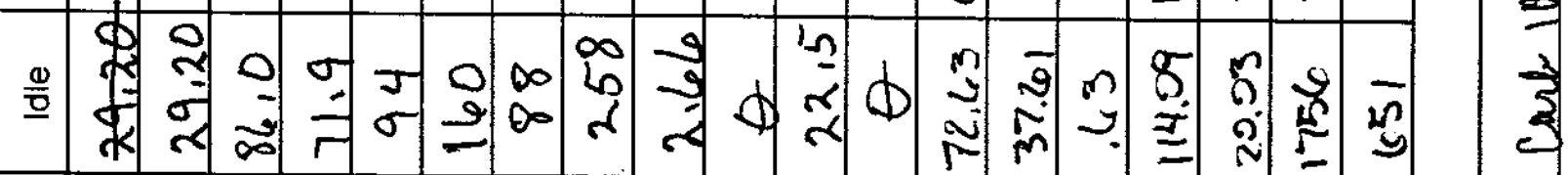

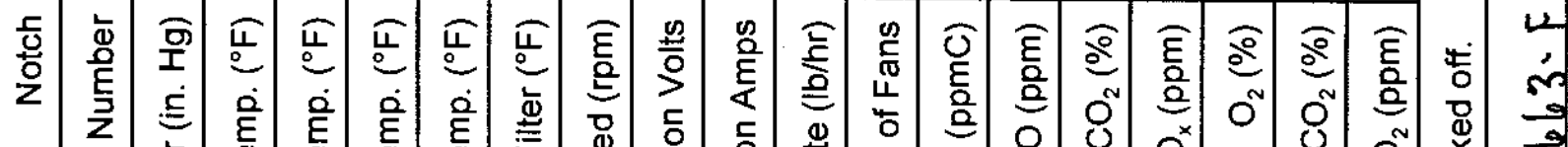

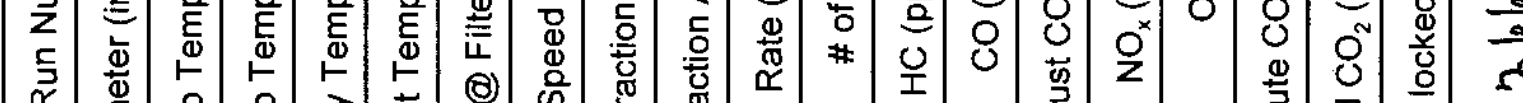

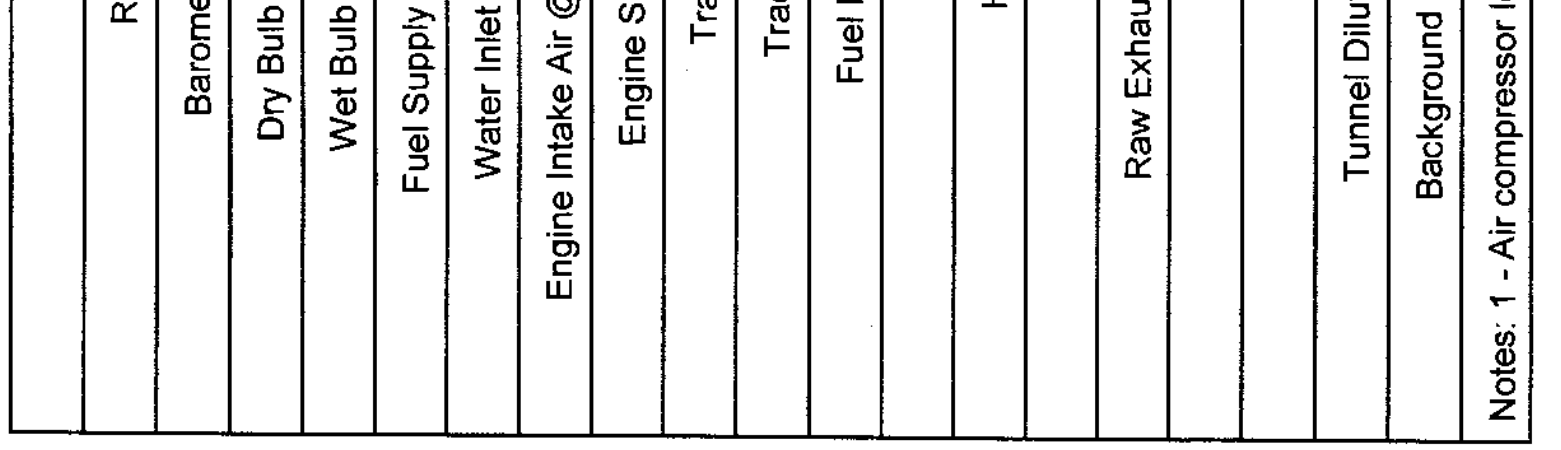
$\sum_{\text {is }}^{\infty}$ $\ddot{\circ}$ 등 준 突 0 


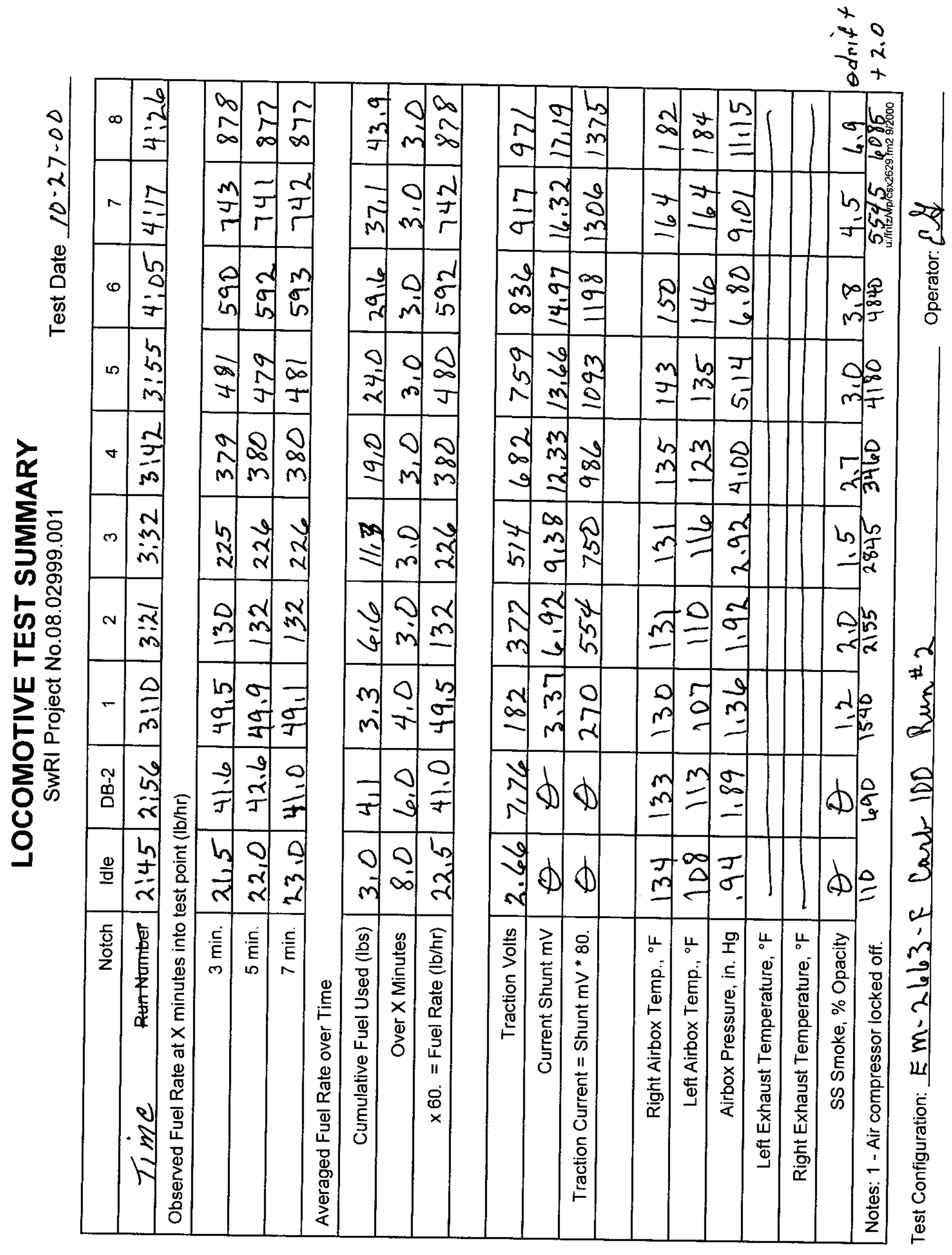




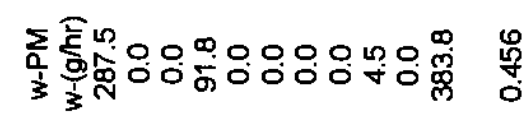

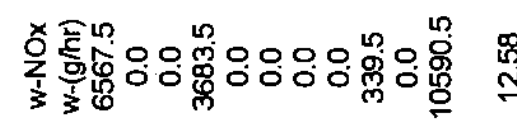

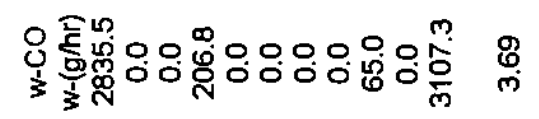

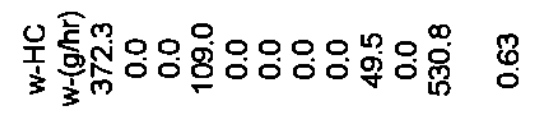

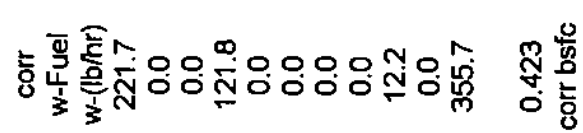

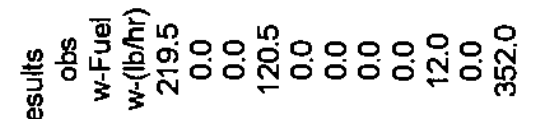

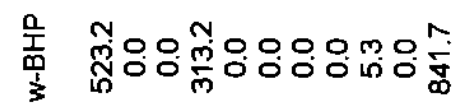

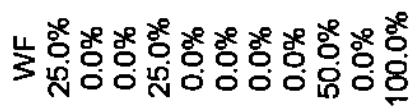

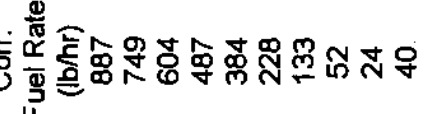

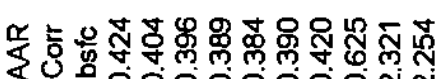

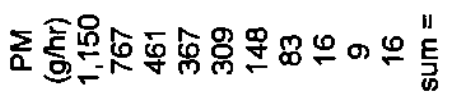

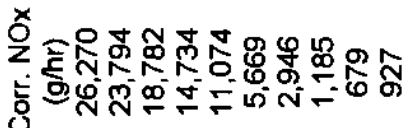

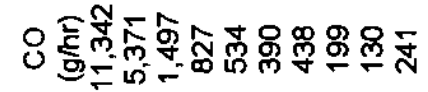

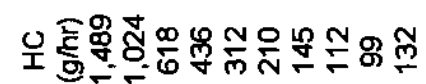

离离

政

논

造语要

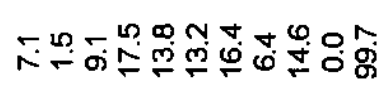

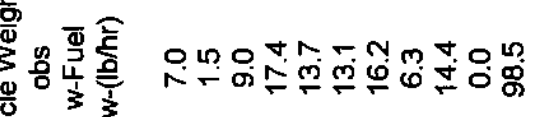

ত্ঠ

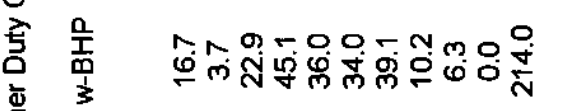

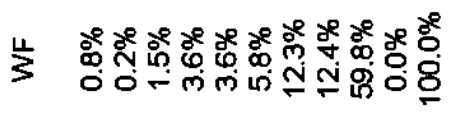

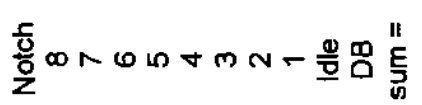

高产

×

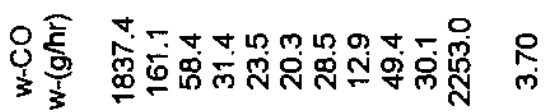

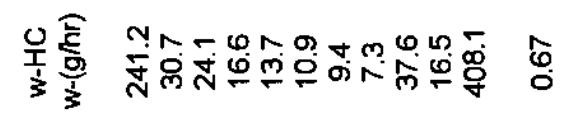

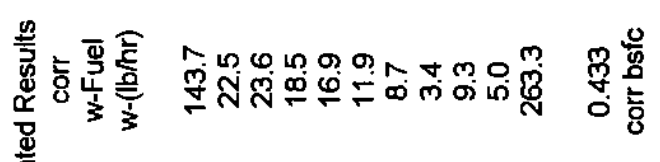

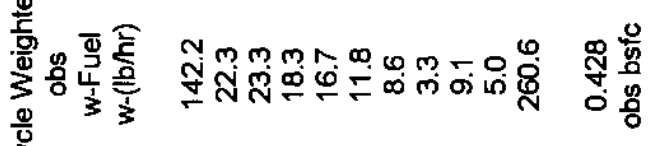

仓

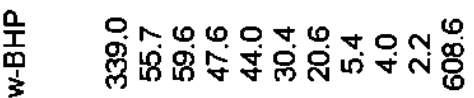

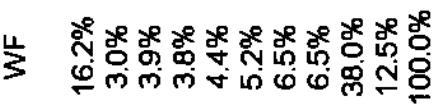




$\begin{array}{ll}\text { LOCOMOTIVE MODEL } & =\text { EMD GP38 } \\ \text { LOCOMOTIVE UNIT \# } & =\text { CSX } \# 2629 \\ \text { THROTTLE NOTCH } & =\text { IDLE } \\ \text { TEST DATE } & =10 / 31 / 0 \\ \text { TEST NUMBER } & =1 / 10 \\ \text { SWRI FUEL CODE } & =\text { EM-2663-F } \\ \text { BAROMETER } & =29.17(\mathrm{in} . \mathrm{Hg}) \\ \text { DRY BULB TEMP } & =86 .\left(^{\circ} \mathrm{F}\right) \\ \text { ABS HUMIDITY } & =101.70(\mathrm{GR} / \mathrm{LB})\end{array}$

ENGINE PERFORMANCE SUMMARY:

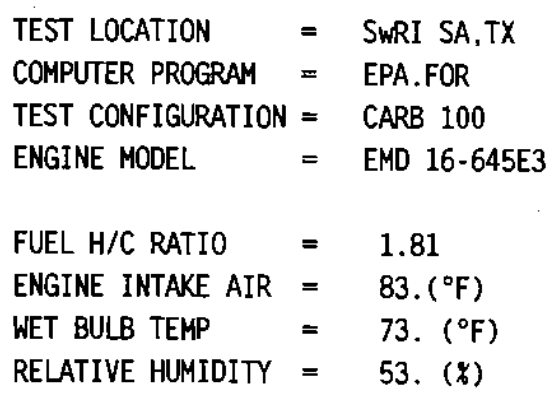

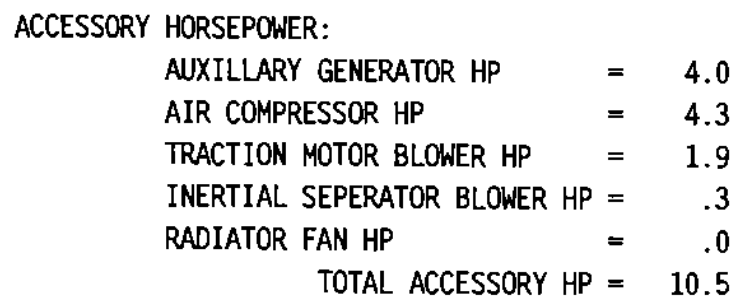

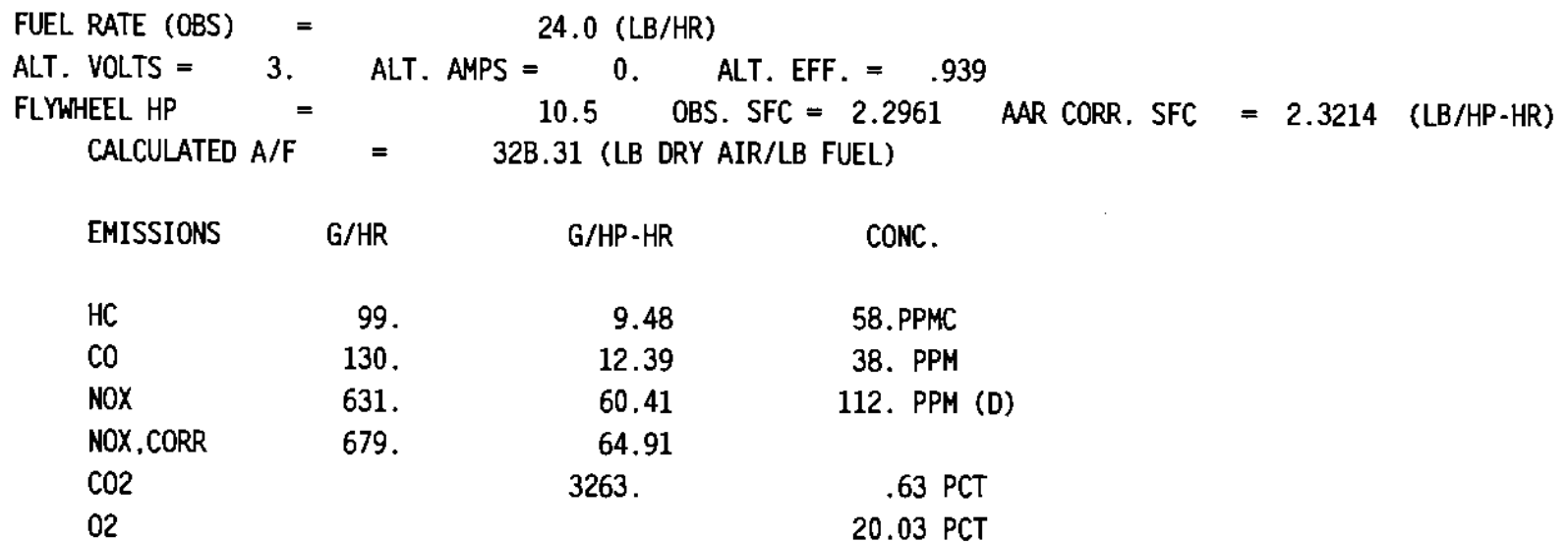

PARTICULATE INFORMATION

FILTER PAIR I.D. NUMBER

$6802.0-87$

FILTER PAIR WEIGHT GAIN (mg) .865

38.815

.022

3.845

.086

9. .862 


$\begin{array}{llll}\text { LOCOMOTIVE MODEL } & =\text { EMD GP38 } & \text { TEST LOCATION } & =\text { SWRI SA,TX } \\ \text { LOCOMOTIVE UNIT \# } & =\text { CSX \#2629 } & \text { COMPUTER PROGRAM } & =\text { EPA.FOR } \\ \text { THROTTLE NOTCH } & =\text { DB2 } & \text { TEST CONFIGURATION }= & \text { CARB } 100 \\ \text { TEST DATE } & =10 / 31 / 0 & \text { ENGINE MODEL } & =\text { EMD } 16-645 E 3 \\ \text { TEST NUMBER } & =2 / 10 & & \\ \text { SWRI FUEL CODE } & =\text { EM-2663-F } & \text { FUEL H/C RATIO } & =1.81 \\ \text { BAROMETER } & =29.17(\mathrm{in} . \mathrm{Hg}) & \text { ENGINE INTAKE AIR }= & 83 .\left({ }^{\circ} \mathrm{F}\right) \\ \text { DRY BULB TEMP } & =85 .\left({ }^{\circ} \mathrm{F}\right) & \text { WET BULB TEMP } & =72 .\left({ }^{\circ} \mathrm{F}\right) \\ \text { ABS HUMIDITY } & =102.40(\mathrm{GR} / \mathrm{LB}) & \text { RELATIVE HUMIDITY }=56 .\left({ }^{\circ}\right)\end{array}$

ENGINE PERFORMANCE SUMMARY:

ACCESSORY HORSEPOWER:

$\begin{array}{rlr}\text { AUXILLARY GENERATOR HP } & =4.0 \\ \text { AIR COMPRESSOR HP } & =6.5 \\ \text { TRACTION MOTOR BLOWER HP } & =6.5 \\ \text { INERTIAL SEPERATOR BLOWER HP } & =1.0 \\ \text { RADIATOR FAN HP } & .0 \\ \text { TOTAL ACCESSORY HP } & =17.9\end{array}$

FUEL RATE (OBS)

ALT. VOLTS =

FLYWHEEL HP

CALCULATED A/F

\section{EMISSIONS}

G/HR

132.

241.

861.

927.

NOX, CORR

$\mathrm{CO} 2$

02

40.0 (LB/HR)

8. ALT. AMPS $=0 . \quad$ ALT. EFF. $=.939$

17.9 OBS. SFC $=2.2289$ AAR CORR. SFC $=2.2535(\mathrm{LB} / \mathrm{HP} \cdot \mathrm{HR})$ 287.71 (LB DRY AIR/LB FUEL)

GONC.

7.37 53.PPMC

$13.42 \quad 48 . \mathrm{PPM}$

48.00 104. PPM (D)

51.67

3171 .

$.72 \mathrm{PCT}$

$19.91 \mathrm{PCT}$

CORRECTION FACTOR SUMMARY:

$\begin{array}{lllll}\text { EPA NOX CF }=1.0763 & \text { NOX-KH } & =1.0624 & \text { NOX-KT } & =1.0000 \\ \text { WET EXH HC CF }=.9886 & \text { AIR TEMP CF }=.9896 & \text { BAROM CF } & =1.0027 \\ \text { FUEL TEMP CF }=.9830 & \text { FUEL S.G. CF }=1.0187 & \text { FUEL HHV CF } & =1.0189\end{array}$

PARTICULATE INFORMATION

FILTER PAIR I.D. NUMBER

$6803.0 \cdot 88$

FILTER PAIR WEIGHT GAIN (mg)

.976

SAMPLE VOLUME (scf)

38.986

DILUTE PM CONCENTRATION (mg/scf)

.025

CO2-BASED DILUTION FACTOR

4.137

.104

PARTICULATE CONCENTRATION IN RAW EXHAUST (mg/scf)

16.

BRAKE-SPECIFIC PARTICULATE EMISSION RATE $(\mathrm{g} / \mathrm{hp}-\mathrm{hr})$ 


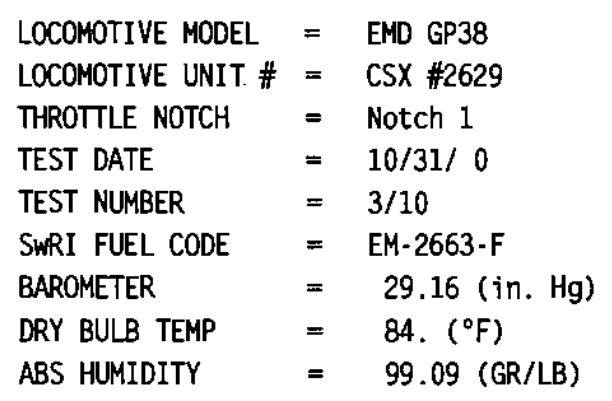

ENGINE PERFORMANCE SUMMARY:

ACCESSORY HORSEPOWER:
AUXILLARY GENERATOR HP $=4.0$
AIR COMPRESSOR HP $=5.3$
TRACTION MOTOR BLOWER HP $=3.5$
INERTIAL SEPERATOR BLOWER HP $=\quad .5$
RADIATOR FAN HP $=.0$
TOTAL ACCESSORY HP $=13.3$

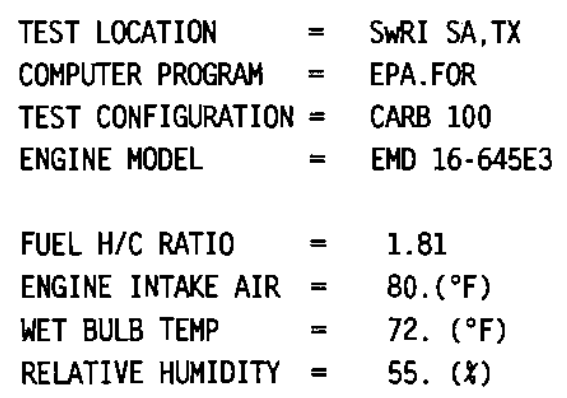

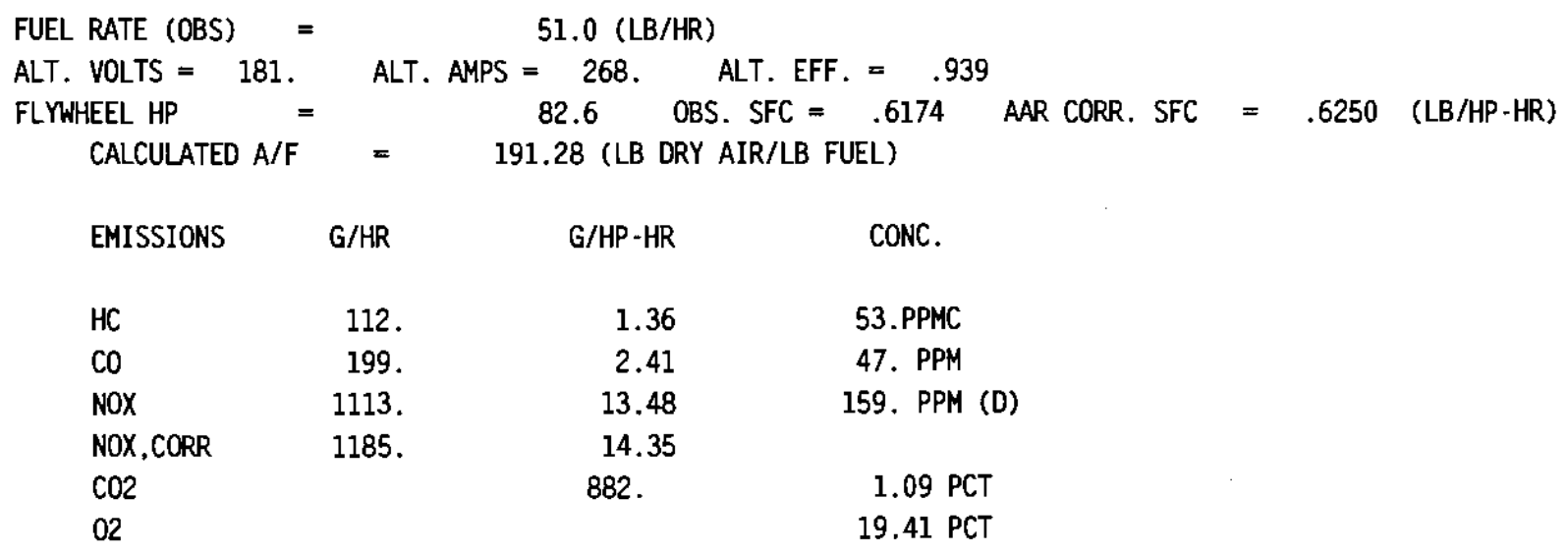

CORRECTION FACTOR SUMMARY:

$\begin{array}{lllll}\text { EPA NOX CF }=1.0646 & \text { NOX-KH } & =1.0529 & \text { NOX-KT } & =1.0000 \\ \text { WET EXH HC CF }=.9855 & \text { AIR TEMP CF }=.9910 & \text { BAROM CF } & =1.0026 \\ \text { FUEL TEMP CF }=.9845 & \text { FUEL S.G. CF }=1.0187 & \text { FUEL HHV CF } & =1.0189\end{array}$

PARTICULATE INFORMATION

FILTER PAIR I.D. NUMBER

$6804.0-89$

FILTER PAIR WEIGHT GAIN (mg)

1.306

SAMPLE VOLUME (SCf)

38.457

.034

3.704

CO2-BASED DILUTION FACTOR

.126

PARTICULATE CONCENTRATION IN RAW EXHAUST ( $\mathrm{mg} / \mathrm{scf}$ )

16.

BRAKE-SPECIFIC PARTICULATE EMISSION RATE $(\mathrm{g} / \mathrm{hp}$ - $\mathrm{hr}$ ) 


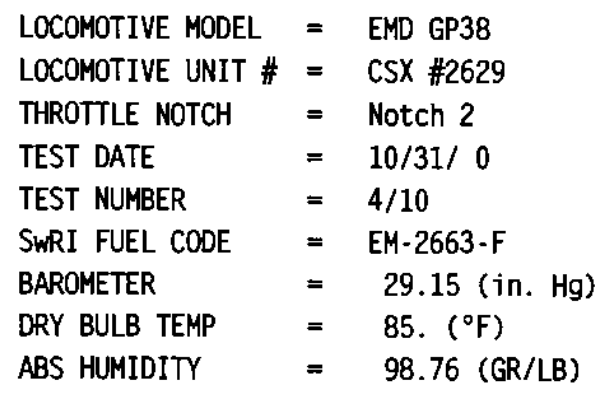

ENGINE PERFORMANCE SUMMARY:

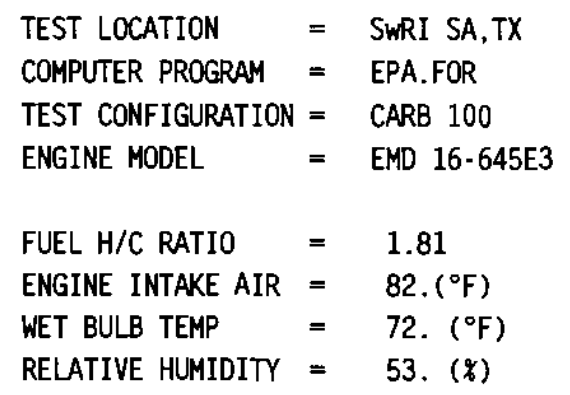

ACCESSORY HORSEPOWER:

$\begin{array}{rlr}\text { AUXILLARY GENERATOR HP } & = & 4.0 \\ \text { AIR COMPRESSOR HP } & = & 6.5 \\ \text { TRACTION MOTOR BLOWER HP } & = & 6.6 \\ \text { INERTIAL SEPERATOR BLOWER HP } & = & 1.0 \\ \text { RADIATOR FAN HP } & = & 3.1 \\ \text { TOTAL ACCESSORY HP } & = & 21.1\end{array}$

FUEL RATE (OBS) $=132.0$ (LB/HR)

ALT. VOLTS $=376 . \quad$ ALT. AMPS $=552 . \quad$ ALT. EFF. $=.939$

FLYWHEEL HP $=317.6$ OBS. SFC $=.4156$ AAR CORR. SFC $=.4203($ LB/HP.HR)

CALCULATED A/F $=95.11$ (LB DRY AIR/LB FUEL)

$\begin{array}{lrrr}\text { EMISSIONS } & \text { G/HR } & \text { G/HP-HR } & \text { CONC. } \\ \text { HC } & 145 . & .46 & 53 . \text { PPMC } \\ \text { CO } & 438 . & 1.38 & 80 . \text { PPM } \\ \text { NOX } & 2782 . & 8.76 & 311 . \text { PPM (D) } \\ \text { NOX, CORR } & 2946 . & 9.28 & \\ \text { C02 } & & 596 . & 2.21 \text { PCT } \\ \text { O2 } & & & 17.78 \text { PCT }\end{array}$

CORRECTION FACTOR SUMMARY:

\begin{tabular}{|c|c|c|c|c|c|}
\hline & 0591 & NOX-KH & $=1.0483$ & NOX-KT & $=1.0000$ \\
\hline $\mathrm{C} C \mathrm{CF}$ & -9757 & AIR TEMP CF & $=.9901$ & BAROM CF & $=1.0025$ \\
\hline UEL & .9850 & FUEL S.G. Cl & $=1.0187$ & FUEL HHV CF & $=1.018$ \\
\hline
\end{tabular}

PARTICULATE INFORMATION

FILTER PAIR I.D. NUMBER

FILTER PAIR WEIGHT GAIN (mg)

$6805.0 \cdot 90$

SAMPLE VOLUME (sCf)

3.853

38.716

DILUTE PM CONCENTRATION (mg/scf)

.100

CO2-BASED DILUTION FACTOR

4.954

PARTICULATE CONCENTRATION IN RAW EXHAUST ( $\mathrm{mg} / \mathrm{scf}$ )

.493

PARTICULATE MASS EMISSION RATE $(\mathrm{g} / \mathrm{hr})$

83.

BRAKE-SPECIFIC PARTICULATE EMISSION RATE $(g / h p-h r)$ 


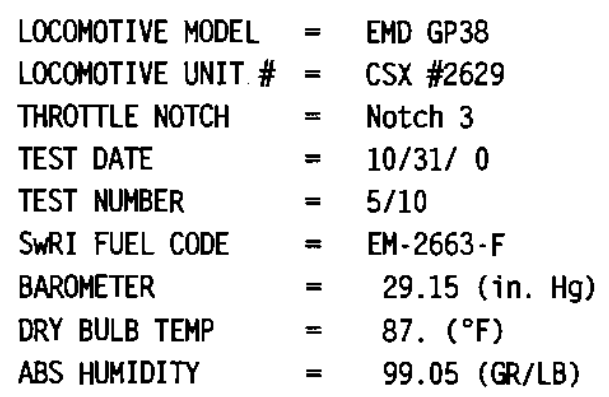

ENGINE PERFORMANCE SUMMARY:

ACCESSORY HORSEPOWER:

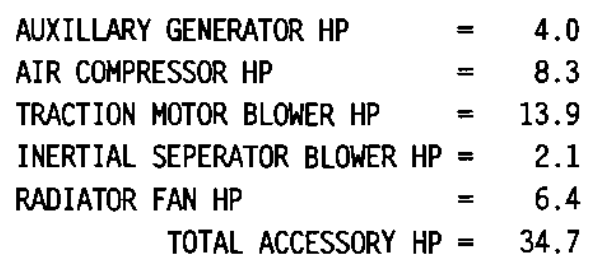

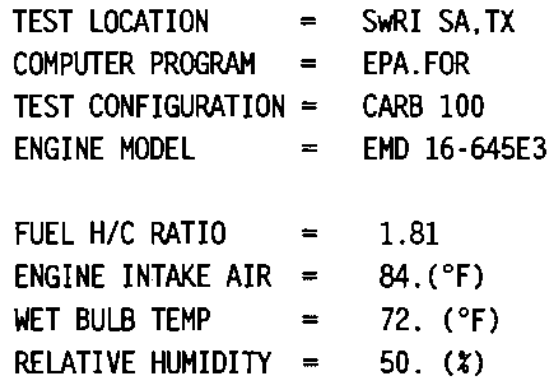
226.0 (LB/HR)

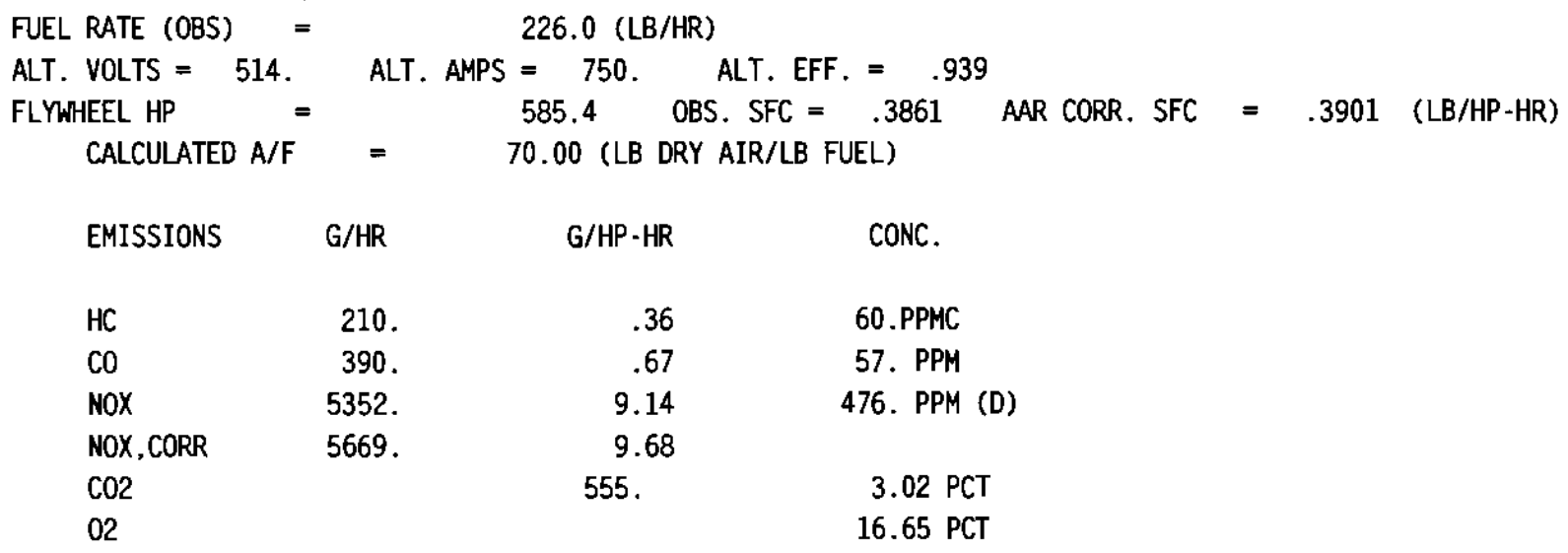

CORRECTION FACTOR SUMMARY:

$\begin{array}{lllll}\text { EPA NOX CF }=1.0592 & \text { NOX-KH } & =1.0484 & \text { NOX-KT } & =1.0000 \\ \text { WET EXH HC CF }=.9688 & \text { AIR TEMP CF }=.9892 & \text { BAROM CF } & =1.0025 \\ \text { FUEL TEMP CF }=.9850 & \text { FUEL S.G. CF }=1.0187 & \text { FUEL HHV CF } & =1.0189\end{array}$

PARTICULATE INFORMATION

FILTER PAIR I.D. NUMBER

$6806.0-91$

5.229

FILTER PAIR WEIGHT GAIN (mg)

38.522

SAMPLE VOLUME (scf)

.136

DILUTE PM CONCENTRATION (mg/scf)

5.090

PARTICULATE CONCENTRATION IN RAW EXHAUST (mg/scf)

.691

PARTICULATE MASS EMISSION RATE $(\mathrm{g} / \mathrm{hr})$

$14 \mathrm{~B}$.

BRAKE-SPECIFIC PARTICULATE EMISSION RATE $(g / h p-h r)$ 


$\begin{array}{ll}\text { LOCOMOTIVE MODEL } & =\text { EMD GP38 } \\ \text { LOCOMOTIVE UNIT \# } & =\text { CSX } \# 2629 \\ \text { THROTTLE NOTCH } & =\text { Notch } 4 \\ \text { TEST DATE } & =10 / 31 / 0 \\ \text { TEST NUMBER } & =6 / 10 \\ \text { SWRI FUEL CODE } & =\text { EM-2663-F } \\ \text { BAROMETER } & =29.14(\mathrm{in} . \mathrm{Hg}) \\ \text { DRY BULB TEMP } & =87 .\left(^{\circ} \mathrm{F}\right) \\ \text { ABS HUMIDITY } & =94.72(\mathrm{GR} / \mathrm{LB})\end{array}$

$\begin{array}{lll}\text { TEST LOCATION } & =\text { SWRI SA,TX } \\ \text { COMPUTER PROGRAM } & =\text { EPA.FOR } \\ \text { TEST CONFIGURATION } & =\text { CARB } 100 \\ \text { ENGINE MODEL } & = & \text { EMD } 16 \cdot 645 E 3 \\ & & \\ \text { FUEL H/C RAT10 } & =1.81 \\ \text { ENGINE INTAKE AIR } & =84 .\left(^{\circ} \mathrm{F}\right) \\ \text { WET BULB TEMP } & =72 .\left(^{\circ} \mathrm{F}\right) \\ \text { RELATIVE HUMIDITY } & =49 .(\%)\end{array}$

ENGINE PERFORMANCE SUMMARY:

ACCESSORY HORSEPOWER:

$\begin{array}{rlr}\text { AUXILLARY GENERATOR HP } & = & 4.0 \\ \text { AIR COMPRESSOR HP } & 9.6 \\ \text { TRACTION MOTOR BLOWER HP } & =20.9 \\ \text { INERTIAL SEPERATOR BLOWER HP } & =3.1 \\ \text { RADIATOR FAN HP } & 9.6 \\ \text { TOTAL ACCESSORY HP } & =47.2\end{array}$

FUEL RATE (OBS) =

$380.0(\mathrm{LB} / \mathrm{HR})$

ALT. VOLTS $=679$.

ALT. AMPS $=983 . \quad$ ALT. EFF. $=.939$

1000.7 OBS. SFC $=.3797$

AAR CORR. SFC $=.3837(\mathrm{LB} / \mathrm{HP}-\mathrm{HR})$

CALCULATED A/F $=47.63$ (LB DRY AIR/LB FUEL)

EMISSIONS G/HR G/HP-HR CONC.

$\begin{array}{llll}H C & 312 . & .31 & 78 . P P M C\end{array}$

$\begin{array}{llll}\mathrm{CO} & 534 . & .53 & 69 . \mathrm{PPM}\end{array}$

$\begin{array}{lll}\text { NOX } & 10566 . & 10.56\end{array}$

NOX, CORR 11074 . 11.07

$\mathrm{CO} 2$

546.

4.47 PCT

02

14.77 PCT

CORRECTION FACTOR SUMMARY:

$\begin{array}{lllll}\text { EPA NOX CF }=1.0481 & \text { NOX-KH } & =1.0394 & \text { NOX-KT } & =1.0000 \\ \text { WET EXH HC CF }=.9568 & \text { AIR TEMP CF }=.9892 & \text { BAROM CF } & =1.0024 \\ \text { FUEL TEMP CF }=.9855 & \text { FUEL S.G. CF }=1.0187 & \text { FUEL HHV CF } & =1.0189\end{array}$

PARTICULATE INFORMATION

FILTER PAIR I.D. NUMBER

$6807.0-92$

FILTER PAIR WEIGHT GAIN (mg)

7.642

SAMPLE VOLUME (scf)

38.164

DILUTE PM CONCENTRATION (mg/scf)

.200

CO2-BASED DILUTION FACTOR

6.282

1.258

PARTICULATE CONCENTRATION IN RAW EXHAUST ( $\mathrm{mg} / \mathrm{scf}$ )

309 .

BRAKE-SPECIFIC PARTICULATE EMISSION RATE $(g / h p-h r)$ 


$\begin{array}{ll}\text { LOCOMOTIVE MODEL } & =\text { EMD GP38 } \\ \text { LOCOMOTIVE UNIT \# } & \text { CSX \#2629 } \\ \text { THROTTLE NOTCH } & =\text { Notch } 5 \\ \text { TEST DATE } & =10 / 31 / 0 \\ \text { TEST NUMBER } & =7 / 10 \\ \text { SWRI FUEL CODE } & =\text { EM-2663-F } \\ \text { BAROMETER } & =29.14(\text { in. Hg) } \\ \text { DRY BULB TEMP } & =84 .\left({ }^{\circ}\right) \\ \text { ABS HUMIDITY } & =94.54(\mathrm{GR} / \mathrm{LB})\end{array}$

ENGINE PERFORMANCE SUMMARY:

ACCESSORY HORSEPOWER:

$\begin{array}{rlr}\text { AUXILLARY GENERATOR HP } & = & 4.0 \\ \text { AIR COMPRESSOR HP } & = & 10.8 \\ \text { TRACTION MOTOR BLOWER HP } & = & 30.2 \\ \text { INERTIAL SEPERATOR BLOWER HP } & = & 4.5 \\ \text { RADIATOR FAN HP } & = & 27.9 \\ \text { TOTAL ACCESSORY HP } & =77.4\end{array}$

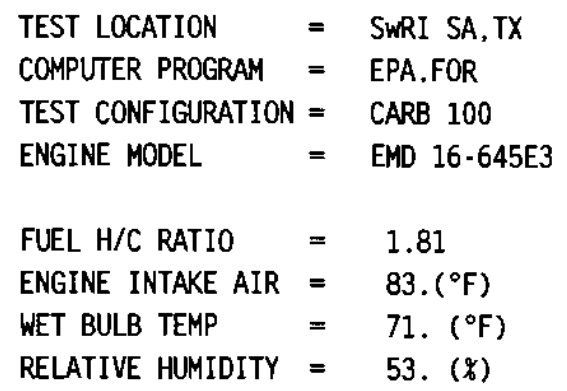

$482.0(\mathrm{LB} / \mathrm{HR})$

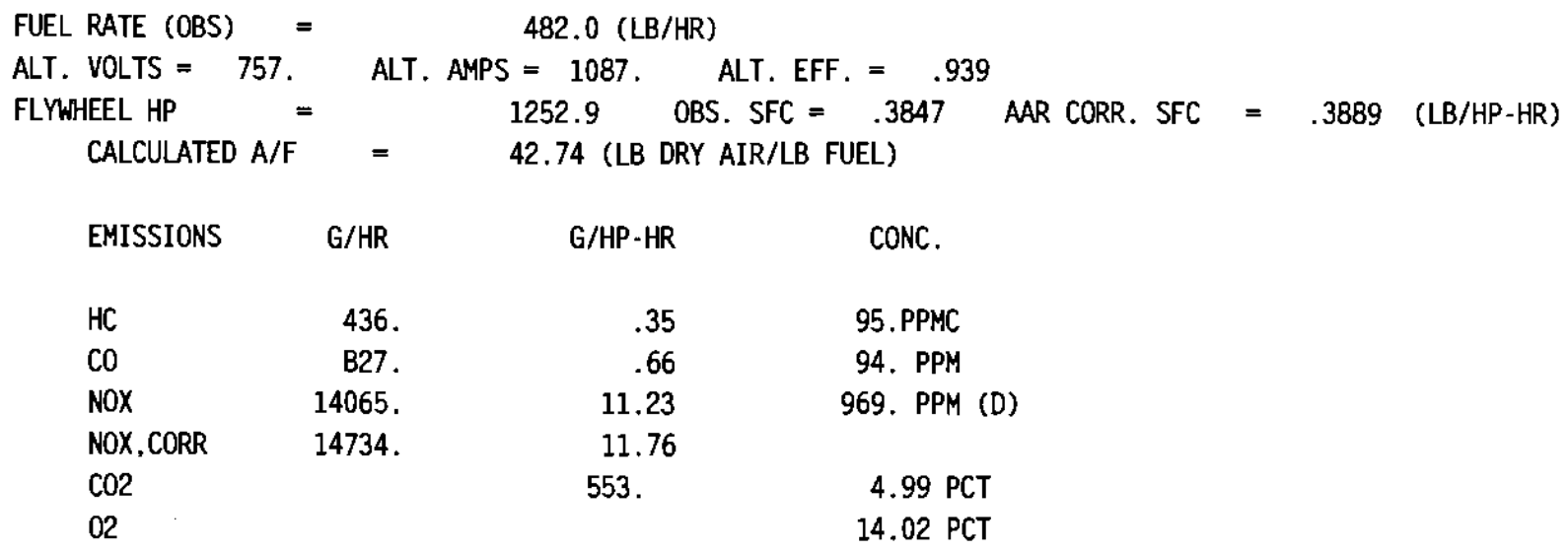

CORRECTION FACTOR SUMMARY:

$\begin{array}{lllll}\text { EPA NOX CF }=1.0476 & \text { NOX-KH } & =1.0389 & \text { NOX-KT } & =1.0000 \\ \text { WET EXH HC CF }=.9525 & \text { AIR TEMP CF }=.9896 & \text { BAROM CF } & =1.0024 \\ \text { FUEL TEMP CF }=.9860 & \text { FUEL S.G. CF }=1.0187 & \text { FUEL HHV CF } & =1.0189\end{array}$

PARTICULATE INFORMATION

FILTER PAIR I.D. NUMBER

6808.0-93

FILTER PAIR WEIGHT GAIN (mg)

7.564

37.472

DILUTE PM CONCENTRATION (mg/scf) .202

CO2-BASED DILUTION FACTOR

6.477

1.307

PARTICULATE CONCENTRATION IN RAW EXHAUST (mg/scf)

PARTICULATE MASS EMISSION RATE $(\mathrm{g} / \mathrm{hr})$

367.

8RAKE-SPECIFIC PARTICULATE EMISSION RATE ( $\mathrm{g} / \mathrm{hp}-\mathrm{hr}$ ) 


$\begin{array}{ll}\text { LOCOMOTIVE MODEL } & =\text { EMD GP38 } \\ \text { LOCOMOTIVE UNIT \# } & =\text { CSX \#2629 } \\ \text { THROTTLE NOTCH } & =\text { Notch } 6 \\ \text { TEST DATE } & =10 / 31 / 0 \\ \text { TEST NUMBER } & =8 / 10 \\ \text { SWRI FUEL CODE } & =\text { EM-2663.F } \\ \text { BAROMETER } & =29.13(\mathrm{in} . \mathrm{Hg}) \\ \text { DRY 8ULB TEMP } & =85 .\left(^{\circ} \mathrm{F}\right) \\ \text { ABS HUMIDITY } & =94.53(\mathrm{GR} / \mathrm{LB})\end{array}$

ENGINE PERFORMANCE SUMMARY:

ACCESSORY HORSEPOWER:

$\begin{array}{llr}\text { AUXILLARY GENERATOR HP } & =4.0 \\ \text { AIR COMPRESSOR HP } & =12.2 \\ \text { TRACTION MOTOR BLOWER HP } & =43.4 \\ \text { INERTIAL SEPERATOR BLOWER HP } & =6.5 \\ \text { RADIATOR FAN HP } & 40.2 \\ \text { TOTAL ACCESSORY HP } & =106.3\end{array}$

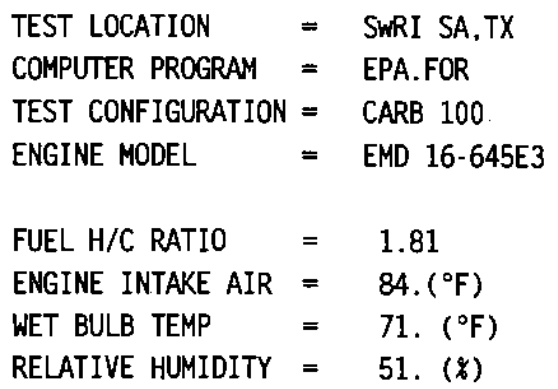

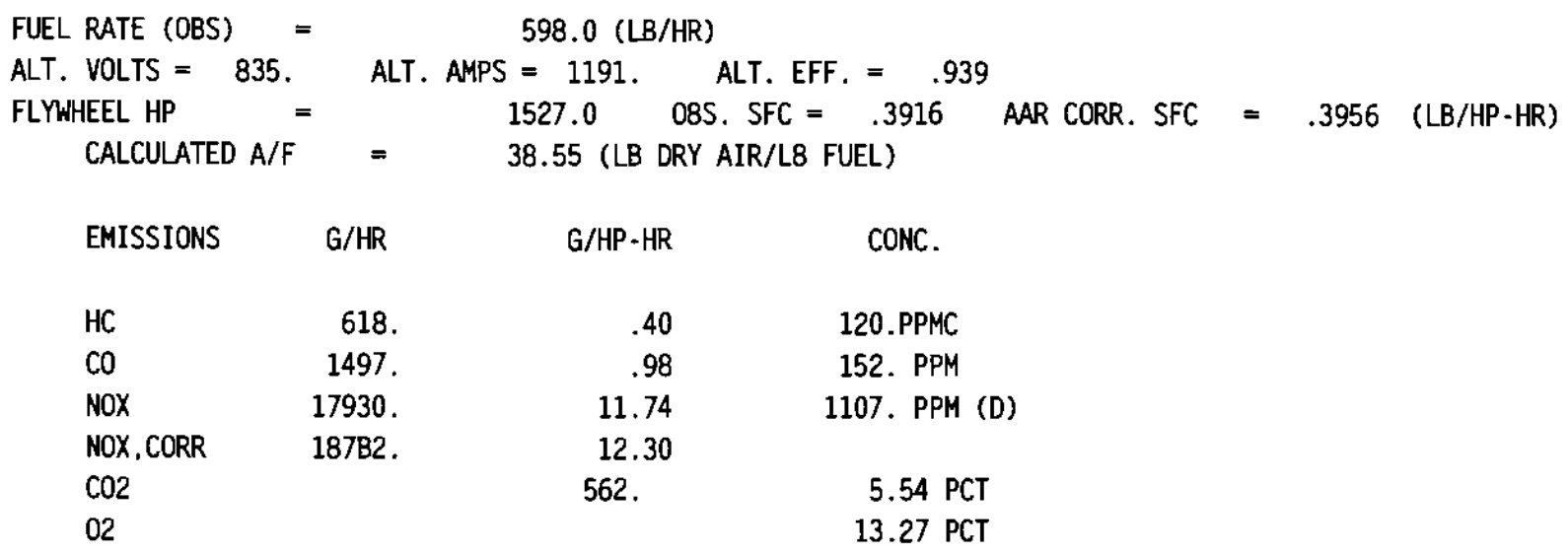

CORRECTION FACTOR SUMMARY:

$\begin{array}{lllll}\text { EPA NOX CF }=1.0475 & \text { NOX-KH } & =1.0389 & \text { NOX-KT } & =1.0000 \\ \text { WET EXH HC CF }=.9480 & \text { AIR TEMP CF }=.9892 & \text { BAROM CF } & =1.0023 \\ \text { FUEL TEMP CF }=.9850 & \text { FUEL S.G. CF }=1.0187 & \text { FUEL HHV CF } & =1.0189\end{array}$

PARTICULATE INFORMATION

FILTER PAIR I.D. NUMBER

$6809.0-94$

FILTER PAIR WEIGHT GAIN (mg)

6.248

37.382

SAMPLE VOLUME (sCf)

.167

DILUTE PM CONCENTRATION (mg/scf)

8.758

PARTICULATE CONCENTRATION IN RAW EXHAUST (mg/scf)

PARTICULATE MASS EMISSION RATE $(\mathrm{g} / \mathrm{hr})$

1.464

BRAKE-SPECIFIC PARTICULATE EMISSION RATE $(g / h p-h r)$

461. 
LOCOMOTIVE EMISSIONS

$\begin{array}{ll}\text { LOCOMOTIVE MODEL } & =\text { EMD GP38 } \\ \text { LOCOMOTIVE UNIT \# } & \text { CSX } \# 2629 \\ \text { THROTLLE NOTCH } & =\text { Notch } 7 \\ \text { TEST DATE } & =10 / 31 / 0 \\ \text { TEST NUMBER } & =9 / 10 \\ \text { SWRI FUEL CODE } & =\text { EM-2663-F } \\ \text { BAROMETER } & =29.13(\mathrm{in} . \mathrm{Hg}) \\ \text { DRY BULB TEMP } & =85 .\left({ }^{\circ} \mathrm{F}\right) \\ \text { ABS HUMIDITY } & =94.83(\mathrm{GR} / \mathrm{LB})\end{array}$

SWRI PROJECT NO. 08-2999-001

ENGINE PERFORMANCE SUMMARY:

ACCESSORY HORSEPOWER:
AUXILLARY GENERATOR HP $\quad=\quad 4.0$
AIR COMPRESSOR HP $\quad=13.8$
TRACTION MOTOR BLOWER HP $=62.8$
INERTIAL SEPERATOR BLOWER HP $=9.4$
RADIATOR FAN HP $\quad=58.1$
TOTAL ACCESSORY HP $=148.2$

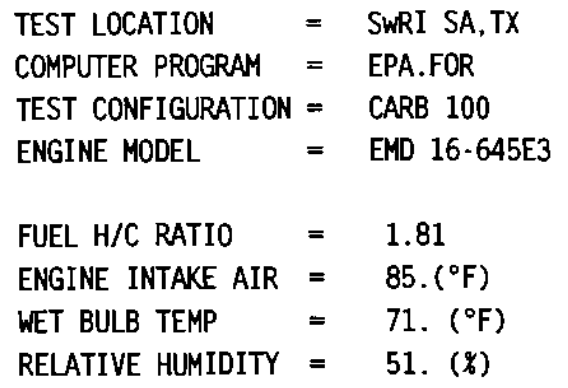

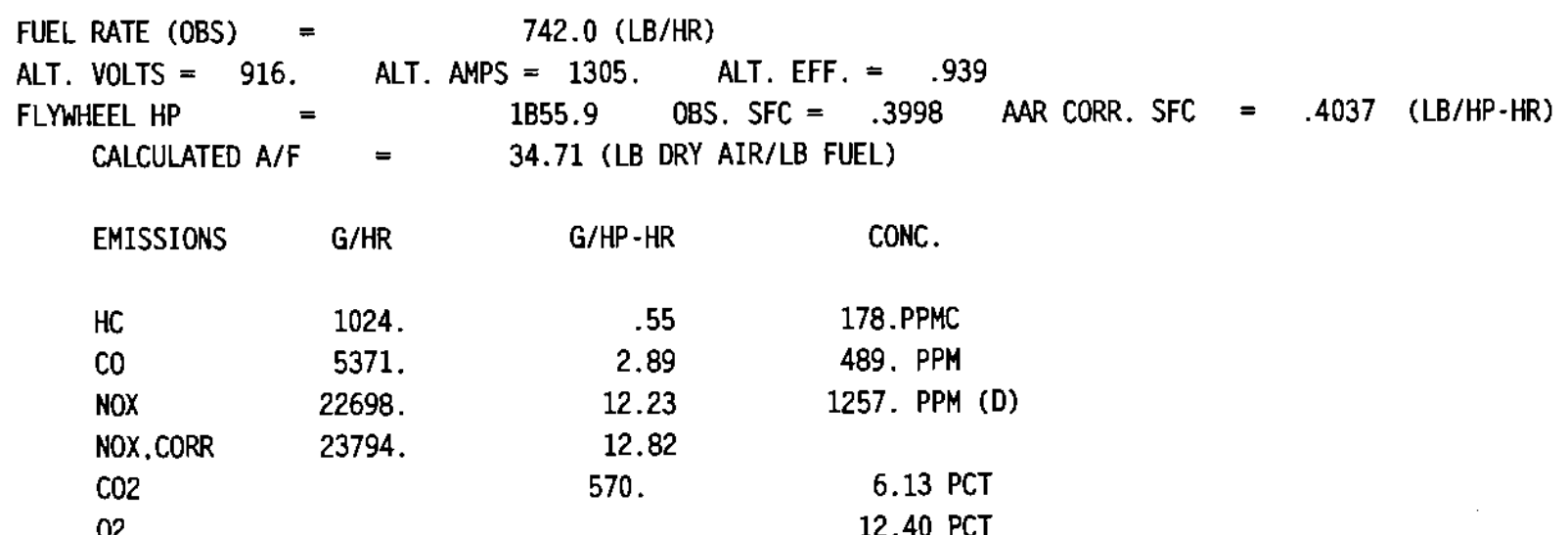


LOCOMOTIVE EMISSIONS SWRI PROJECT NO. 08-2999-001

\begin{tabular}{|c|c|c|c|c|}
\hline LOCOMOTIVE MODEL & $=E M D G P 38$ & TEST LOCATION & $=$ & SWRI SA,TX \\
\hline LOCOMOTIVE UNIT \# & CSX \#2629 & COMPUTER PROGRAM & $=$ & EPA.FOR \\
\hline THROTTLE NOTCH & Notch 8 & TEST CONFIGURATION & $=$ & CARB 100 \\
\hline TEST DATE & $10 / 31 / 0$ & ENGINE MODEL & $=$ & EMD $16 \cdot 645 E 3$ \\
\hline TEST NUMBER & $10 / 10$ & & & \\
\hline SWRI FUEL CODE & $E M-2663-F$ & FUEL H/C RATIO & $=$ & 1.81 \\
\hline BAROMETER & $=29.13($ in. $\mathrm{Hg})$ & ENGINE INTAKE AIR & $=$ & $84 .\left({ }^{\circ} \mathrm{F}\right)$ \\
\hline DRY BULB TEMP & 84. $\left({ }^{\circ} \mathrm{F}\right)$ & WET BULB TEMP & $=$ & 71. $\left({ }^{\circ} \mathrm{F}\right)$ \\
\hline ABS HUMIDITY & 95.21 (GR/LB) & RELATIVE HUMIDITY & $=$ & 52. (\%) \\
\hline
\end{tabular}

ENGINE PERFORMANCE SUMMARY:

ACCESSORY HORSEPOWER:

$\begin{array}{rrr}\text { AUXILLARY GENERATOR HP } & =4.0 \\ \text { AIR COMPRESSOR HP } & =15.1 \\ \text { TRACTION MOTOR BLOWER HP } & =82.8 \\ \text { INERTIAL SEPERATOR BLOWER HP } & =12.4 \\ \text { RADIATOR FAN HP } & 76.6 \\ \text { TOTAL ACCESSORY HP } & =190.8\end{array}$

FUEL RATE (OBS) $=878.0$ (LB/HR)

ALT. VOLTS $=969 . \quad$ ALT. AMPS $=1374 . \quad$ ALT. EFF. $=.939$

FLYWHEEL HP $=2092.8$ OBS. SFC $=.4195$ AAR CORR. SFC $=.4238$ (L8/HP-HR)

CALCULATED A/F $=31.80$ (LB DRY AIR/LB FUEL)

$\begin{array}{lrrr}\text { EMISSIONS } & \text { G/HR } & \text { G/HP.HR } & \text { CONC. } \\ \text { HC } & 1489 . & .71 & 238 . \text { PPMC } \\ \text { CO } & 11342 . & 5.42 & 954 . \text { PPM } \\ \text { NOX } & 25038 . & 11.96 & 1282 . \text { PPM (D) } \\ \text { NOX, CORR } & 26270 . & 12.55 & \\ \text { CO2 } & & 594 . & 6.66 \text { PCT } \\ 02 & & & 11.64 \text { PCT }\end{array}$

CORRECTION FACTOR SUMMARY:

$\begin{array}{lllll}\text { EPA NOX CF }=1.0492 & \text { NOX-KH } & =1.0403 & \text { NOX-KT } & =1.0000 \\ \text { WET EXH HC CF }=.9384 & \text { AIR TEMP CF }=.9892 & \text { BAROM CF } & =1.0023 \\ \text { FUEL TEMP CF }=.9850 & \text { FUEL S.G. CF }=1.0187 & \text { FUEL HHV CF } & =1.0189\end{array}$

PARTICULATE INFORMATION

FILTER PAIR I.D. NUMBER

6811.0 .96

FILTER PAIR WEIGHT GAIN (mg)

16.047

SAMPLE VOLUME (SCf)

35.408

DILUTE PM CONCENTRATION (mg/scf)

.453

CO2-BASED DILUTION FACTOR

6.620

3.000

PARTICULATE CONCENTRATION IN RAW EXHAUST (mg/scf)

PARTICULATE MASS EMISSION RATE $(\mathrm{g} / \mathrm{hr})$

1150.

BRAKE-SPECIFIC PARTICULATE EMISSION RATE $(g / h p-h r)$ 


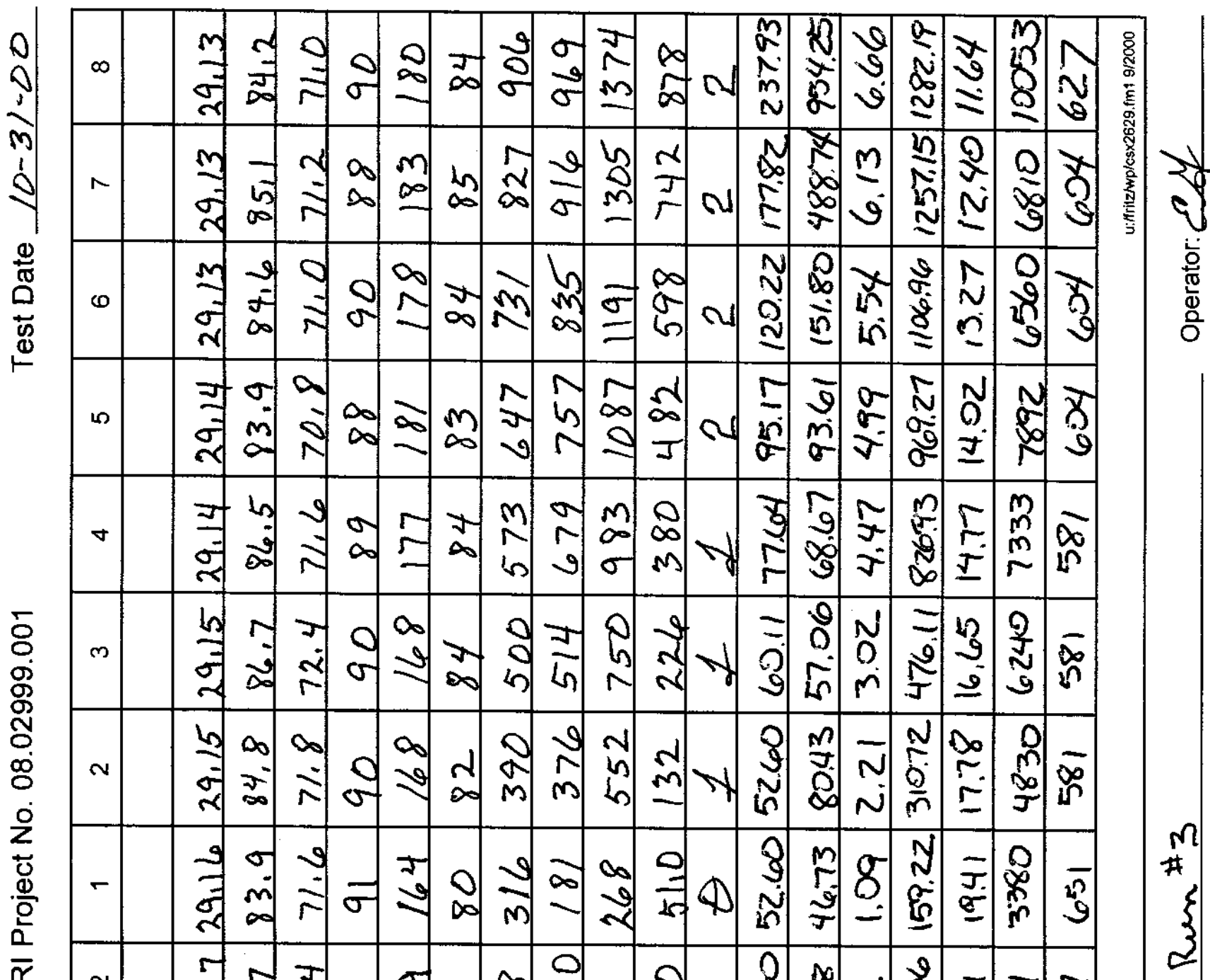

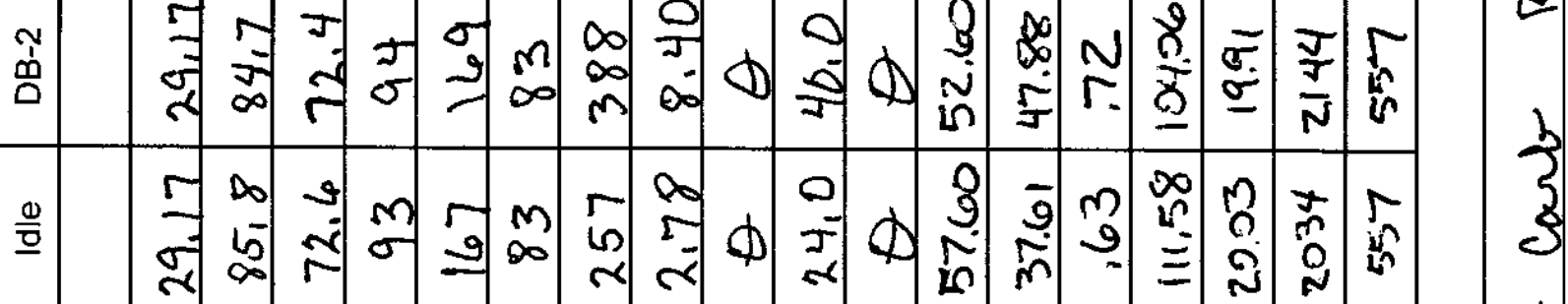

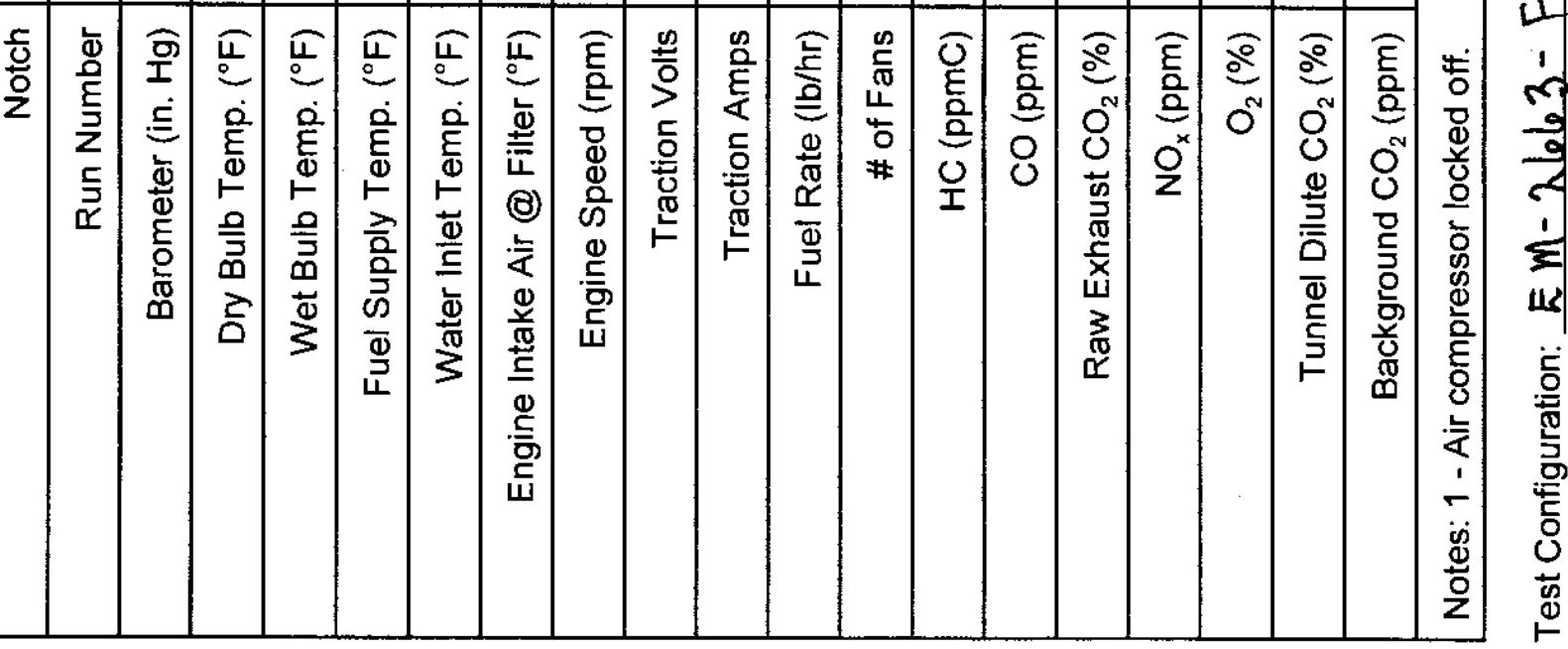




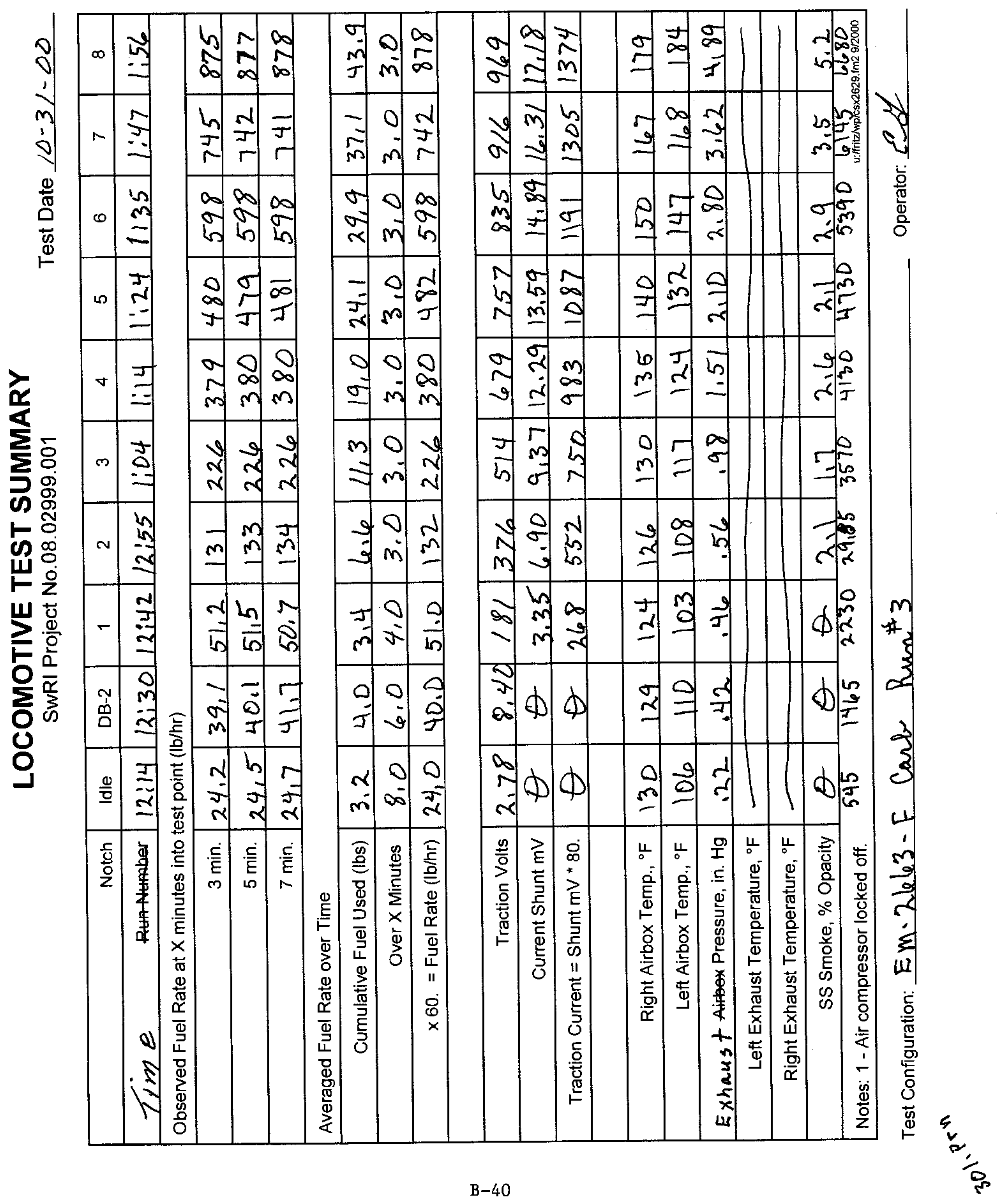




\section{APPENDIX C}

\section{B20 TEST RESULTS}




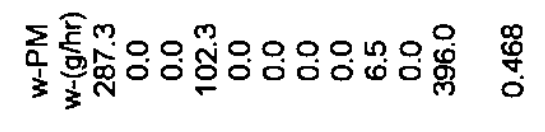

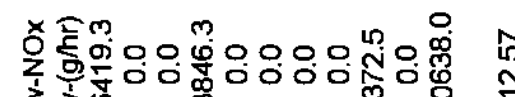

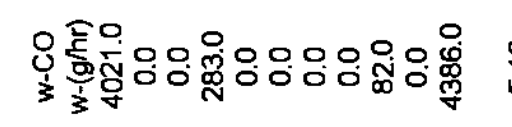

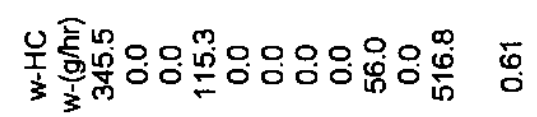

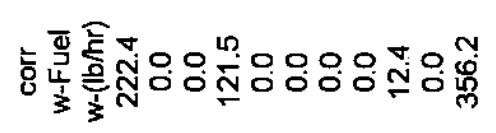

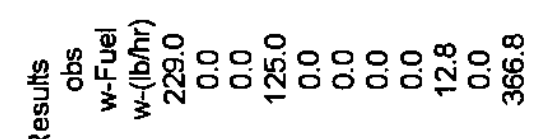

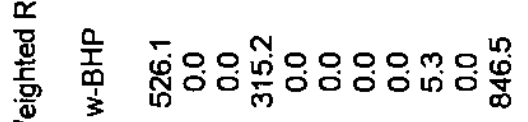

要

㠃

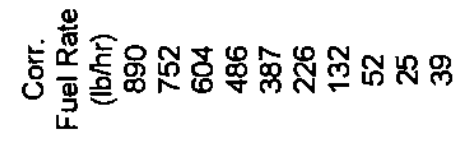

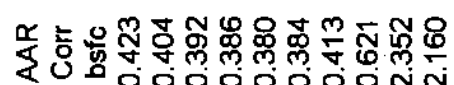

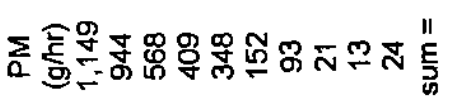

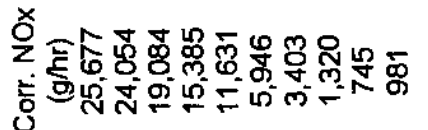

妥

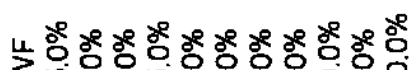

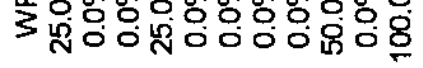

实䐱

范葛

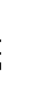

(1)

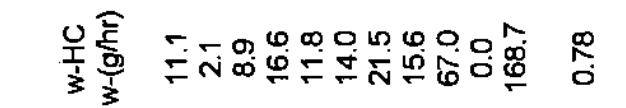

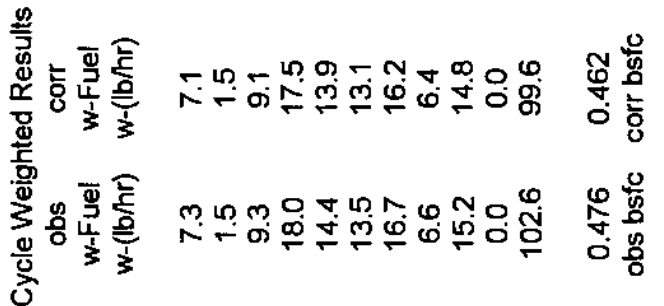

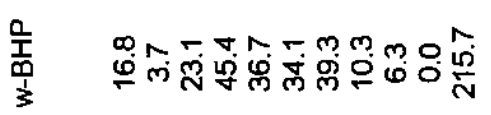

५

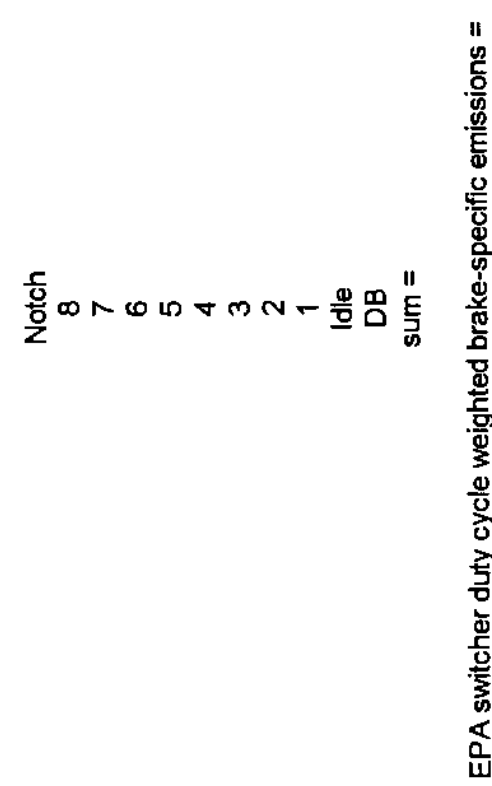

总

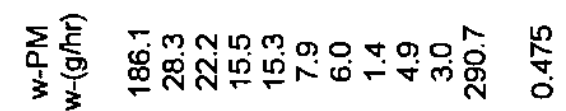

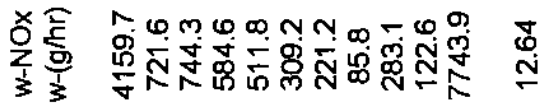

O

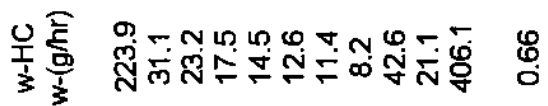

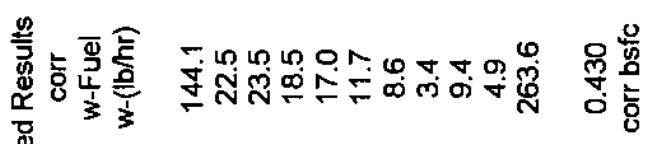

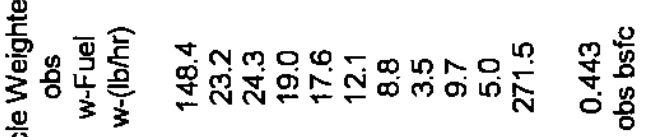

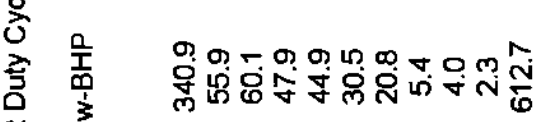

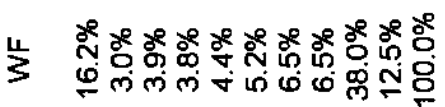

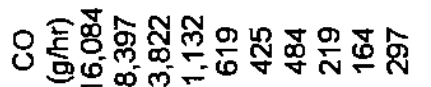

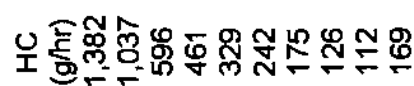

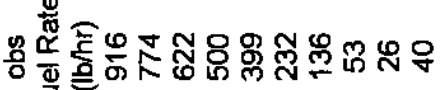

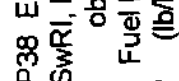

通

온

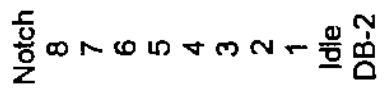


LOCOMOTIVE EMISSIONS SWRI PROJECT NO. 08-2999-001

$\begin{array}{lll}\text { LOCOMOTIVE MODEL } & =\text { EMD GP38 } \\ \text { LOCOMOTIVE UNIT \# } & =\text { CSX } \# 2629 \\ \text { THROTTLE NOTCH } & =\text { IDLE } \\ \text { TEST DATE } & =10 / 26 / 0 \\ \text { TEST NUMBER } & =1 / 10 \\ \text { SWRI FUEL CODE } & =\text { EM }-3004-\mathrm{F} \\ \text { BAROMETER } & =29.15(\mathrm{in} . \mathrm{Hg}) \\ \text { DRY BULB TEMP } & =83 .\left(^{\circ} \mathrm{F}\right) \\ \text { ABS HUMIDITY } & =99.91(\mathrm{GR} / \mathrm{LB})\end{array}$

ENGINE PERFORMANCE SUMMARY:

ACCESSORY HORSEPOWER:

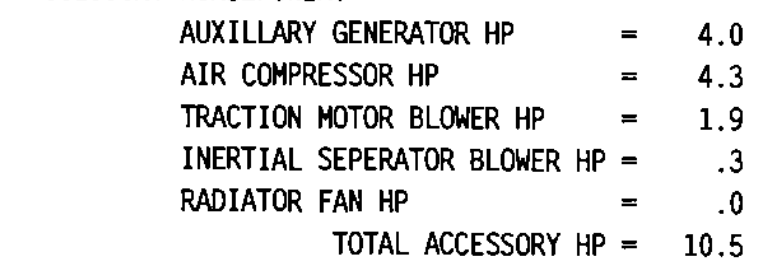

$\begin{array}{llr}\text { AUXILLARY GENERATOR HP } & = & 4.0 \\ \text { AIR COMPRESSOR HP } & = & 4.3 \\ \text { TRACTION MOTOR BLOWER HP } & =1.9 \\ \text { INERTIAL SEPERATOR BLOWER HP } & =.3 \\ \text { RADIATOR FAN HP } & =0 \\ \text { TOTAL ACCESSORY HP } & =10.5\end{array}$

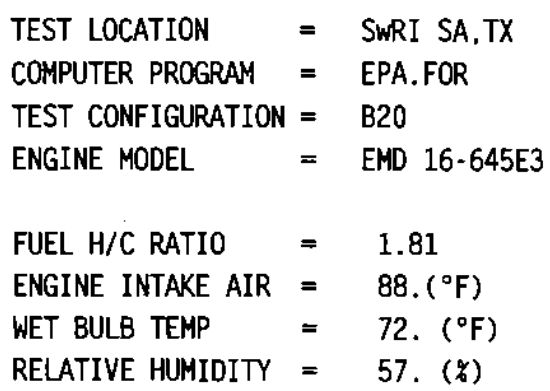

PARTICULATE INFORMATION

FILTER PAIR I.D. NUMBER

$6722.0-7$

FILTER PAIR WEIGHT GAIN (mg)

1.046

SAMPLE VOLUME (scf)

38.926

DILUTE PM CONCENTRATION (mg/scf)

.027

CO2-BASED DILUTION FACTOR

4.560

PARTICULATE CONCENTRATION IN RAW EXHAUST ( $\mathrm{mg} / \mathrm{scf}$ )

PARTICULATE MASS EMISSION RATE $(\mathrm{g} / \mathrm{hr})$

.123

13.

BRAKE-SPECIFIC PARTICULATE EMISSION RATE $(g / h p-h r)$

1.281 


\begin{tabular}{|c|c|c|c|}
\hline LOCOMOTIVE MODEL & $=\quad \mathrm{EMD} \mathrm{GP38}$ & TEST LOCATION & SWRI SA, TX \\
\hline LOCOMOTIVE UNIT \# & $=\quad \operatorname{csx} \# 2629$ & COMPUTER PROGRAM & EPA.FOR \\
\hline THROTTLE NOTCH & DB2 & TEST CONFIGURATION & $\mathrm{B} 20$ \\
\hline TEST DATE & $10 / 26 / 0$ & ENGINE MODEL & EMD $16-645 E 3$ \\
\hline TEST NUMBER & $2 / 10$ & & \\
\hline SWRI FUEL CODE & $E M-3004-F$ & FUEL H/C RATIO & 1.81 \\
\hline BAROMETER & 29.14 (in. $\mathrm{Hg}$ ) & ENGINE INTAKE AIR & $86 .\left({ }^{\circ} \mathrm{F}\right)$ \\
\hline DRY BULB TEMP & 83. $\left({ }^{\circ} \mathrm{F}\right)$ & WET BULB TEMP & 71. $\left({ }^{\circ} \mathrm{F}\right)$ \\
\hline ABS HUMIDITY & $99.37(G R / L B)$ & RELATIVE HUMIDITY & 57. (\%) \\
\hline
\end{tabular}

ENGINE PERFDRMANCE SUMMARY:

ACCESSORY HORSEPOWER:

$\begin{array}{rlr}\text { AUXILLARY GENERATOR HP } & =4.0 \\ \text { AIR COMPRESSOR HP } & =6.5 \\ \text { TRACTION MOTOR BLOWER HP } & =6.5 \\ \text { INERTIAL SEPERATOR BLOWER HP } & =1.0 \\ \text { RADIATOR FAN HP } & .0 \\ \text { TOTAL ACCESSORY HP } & =18.0\end{array}$

FUEL RATE (OBS) $=40.0(\mathrm{LB} / \mathrm{HR})$

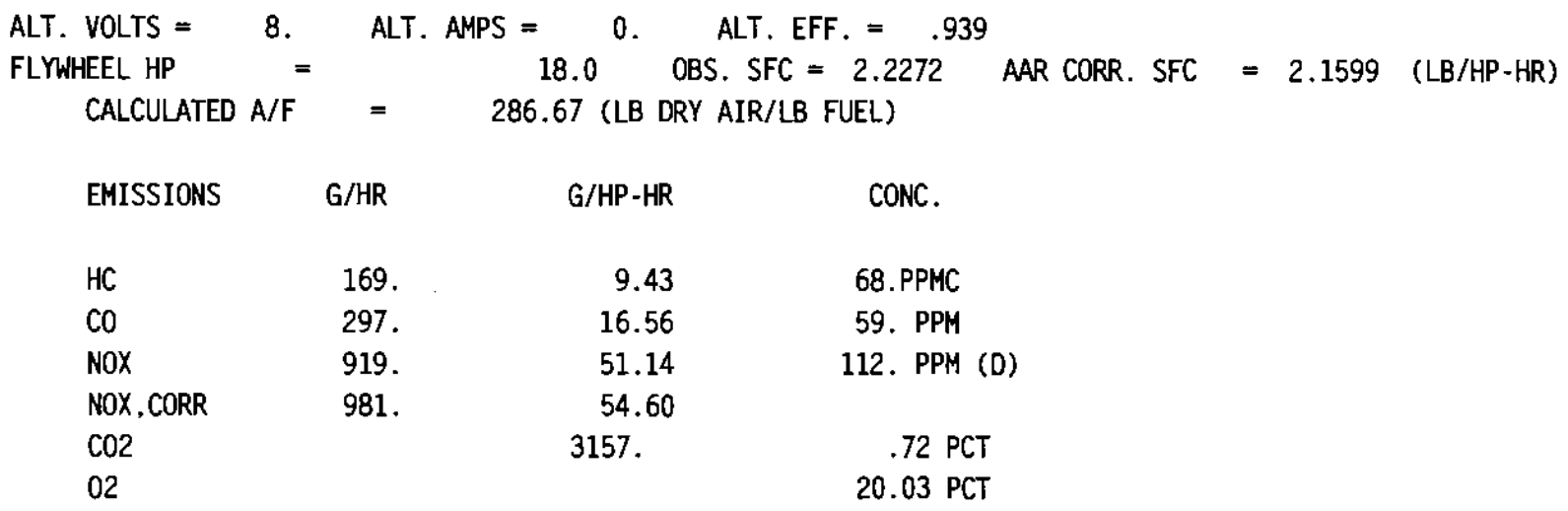

CORRECTION FACTOR SUMMARY:

$\begin{array}{lllll}\text { EPA NOX CF }=1.0676 & \text { NOX-KH } & =1.0553 & \text { NOX-KT } & =1.0000 \\ \text { WET EXH HC CF }=.9888 & \text { AIR TEMP CF }=.9883 & \text { BAROM CF } & =1.0024 \\ \text { FUEL TEMP CF }=.9860 & \text { FUEL S.G. CF }=.9832 & \text { FUEL HHV CF } & =.9789\end{array}$

PARTICULATE INFORMATION

FILTER PAIR I.D. NUMBER

$6723.0-8$

FILTER PAIR WEIGHT GAIN (mg)

1.416

SAMPLE VDLUME (scf)

3B. 756

DILUTE PM CONCENTRATION (mg/scf)

.037

4.246

CD2-BASED DILUTION FACTOR

.155

PARTICULATE CONCENTRATION IN RAW EXHAUST (mg/scf)

PARTICULATE MASS EMISSION RATE $(\mathrm{g} / \mathrm{hr})$

24.

BRAKE-SPECIFIC PARTICULATE EMISSION RATE $(g / h p-h r)$ 
LOCOMOTIVE EMISSIONS SWRI PROJECT NO. 08-2999-001

$\begin{array}{lll}\text { LOCOMOTIVE MODEL } & =\text { EMD GP38 } \\ \text { LOCOMOTIVE UNIT \# } & =\text { CSX } \# 2629 \\ \text { THROTTLE NOTCH } & =\text { Notch } 1 \\ \text { TEST DATE } & =10 / 26 / 0 \\ \text { TEST NUMBER } & =3 / 10 \\ \text { SWRI FUEL CODE } & =\text { EM-3004-F } \\ \text { BAROMETER } & =29.14(\mathrm{jn} . \mathrm{Hg}) \\ \text { DRY BULB TEMP } & =85 .\left({ }^{\circ} \mathrm{F}\right) \\ \text { ABS HUMIDITY } & =96.81(\mathrm{GR} / \mathrm{LB})\end{array}$

$\begin{array}{ll}\text { TEST LOCATION } & \text { SWRI SA, TX } \\ \text { COMPUTER PROGRAM } & =\text { EPA.FOR } \\ \text { TEST CONFIGURATION } & =\text { B20 } \\ \text { ENGINE MODEL } & =\text { EMD } 16-645 E 3 \\ \text { FUEL H/C RATIO } & =1.81 \\ \text { ENGINE INTAKE AIR } & =84 .\left(^{\circ} \mathrm{F}\right) \\ \text { WET BULB TEMP } & =71 .\left({ }^{\circ} \mathrm{F}\right) \\ \text { RELATIVE HUMIDITY } & =53 .(\%)\end{array}$

ENGINE PERFORMANCE SUMMARY:

ACCESSORY HORSEPOWER:

$\begin{array}{rlr}\text { AUXILLARY GENERATOR HP } & =4.0 \\ \text { AIR COMPRESSOR HP } & =5.3 \\ \text { TRACTION MOTOR BLOWER HP } & =3.5 \\ \text { INERTIAL SEPERATOR BLOWER HP } & =.5 \\ \text { RADIATOR FAN HP } & =0 \\ \text { TOTAL ACCESSORY HP } & =13.2\end{array}$

FUEL RATE (OBS) = 53.2 (LB/HR)

ALT. VOLTS $=1 B 2 . \quad A L T$. AMPS $=269 . \quad$ ALT. EFF. $=.939$

FLYWHEEL HP $=83.2$ OBS. SFC $=.6395$ AAR CORR. SFC $=.6208$ (LB/HP-HR)

CALCULATED A/F $=1 B 7.74$ (LB DRY AIR/LB FUEL)

EMISSIONS G/HR G/HP-HR CONC.

$\begin{array}{lrrr}\text { HC } & 126 . & 1.51 & 5 B . P P M C \\ \text { CO } & 219 . & 2.63 & 50 . \text { PPM } \\ \text { NOX } & 1247 . & 14.99 & 174 . \text { PPM (D) } \\ \text { NOX,CORR } & 1320 . & 15.87 & \\ \text { CO2 } & & 914 . & 1.11 \text { PCT } \\ 02 & & & 19.53 \text { PCT }\end{array}$

CORRECTION FACTOR SUMMARY:

EPA NOX CF $=1.0582$

WET EXH HC CF $=.9855$

NOX-KH $=1.0476$

AIR TEMP CF $=.9892$

NOX-KT $=1.0000$

FUEL TEMP CF $=.9855$

FUEL S.G. $C F=.9832$

BAROM CF $=1.0024$

FUEL HHV CF $=.9789$

PARTICULATE INFORMATION

FILTER PAIR I.D. NUMBER

$6724.0-9$

FILTER PAIR WEIGHT GAIN (mg)

1.619

SAMPLE VOLUME (scf)

38.119

DILUTE PH CONCENTRATION (mg/scf)

.042

CO2-BASED DILUTION FACTOR

3.787

PART1CULATE CONCENTRATION IN RAW EXHAUST (mg/scf)

PARTICULATE MASS EMISSION RATE $(\mathrm{g} / \mathrm{hr})$

.161

21.

BRAKE-SPECIFIC PARTICULATE EMISSION RATE $(\mathrm{g} / \mathrm{hp}-\mathrm{hr})$ 


$\begin{array}{lll}\text { LOCOMOTIVE MODEL } & =\text { EMD GP38 } \\ \text { LOCOMOTIVE UNIT \# } & =\text { CSX \#2629 } \\ \text { THROTTLE NOTCH } & =\text { Notch } 2 \\ \text { TEST DATE } & =10 / 26 / 0 \\ \text { TEST NUMBER } & =4 / 10 \\ \text { SWRI FUEL CODE } & =\text { EM-3004-F } \\ \text { BAROMETER } & =29.14(\mathrm{in} . \mathrm{Hg}) \\ \text { DRY BULB TEMP } & =83 .\left(^{\circ} \mathrm{F}\right) \\ \text { ABS HUMIDITY } & =97.63(\mathrm{GR} / \mathrm{LB})\end{array}$

ENGINE PERFORMANCE SUMMARY:

ACCESSORY HORSEPOWER:

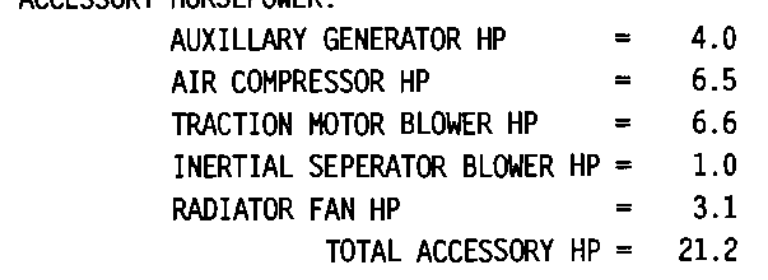

$\begin{array}{rlr}\text { AUXILLARY GENERATOR HP } & =4.0 \\ \text { AIR COMPRESSOR HP } & =6.5 \\ \text { TRACTION MOTOR BLOWER HP } & =6.6 \\ \text { INERTIAL SEPERATOR BLOWER HP } & =1.0 \\ \text { RADIATOR FAN HP } & =3.1 \\ \text { TOTAL ACCESSORY HP } & =21.2\end{array}$

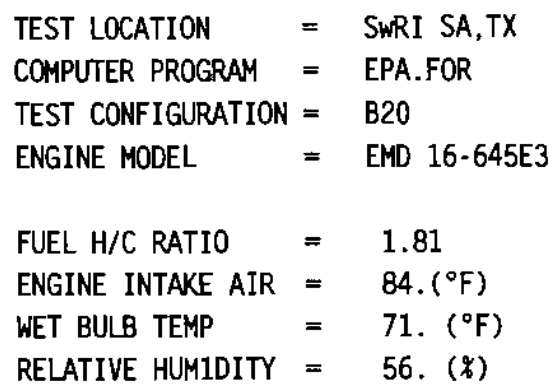

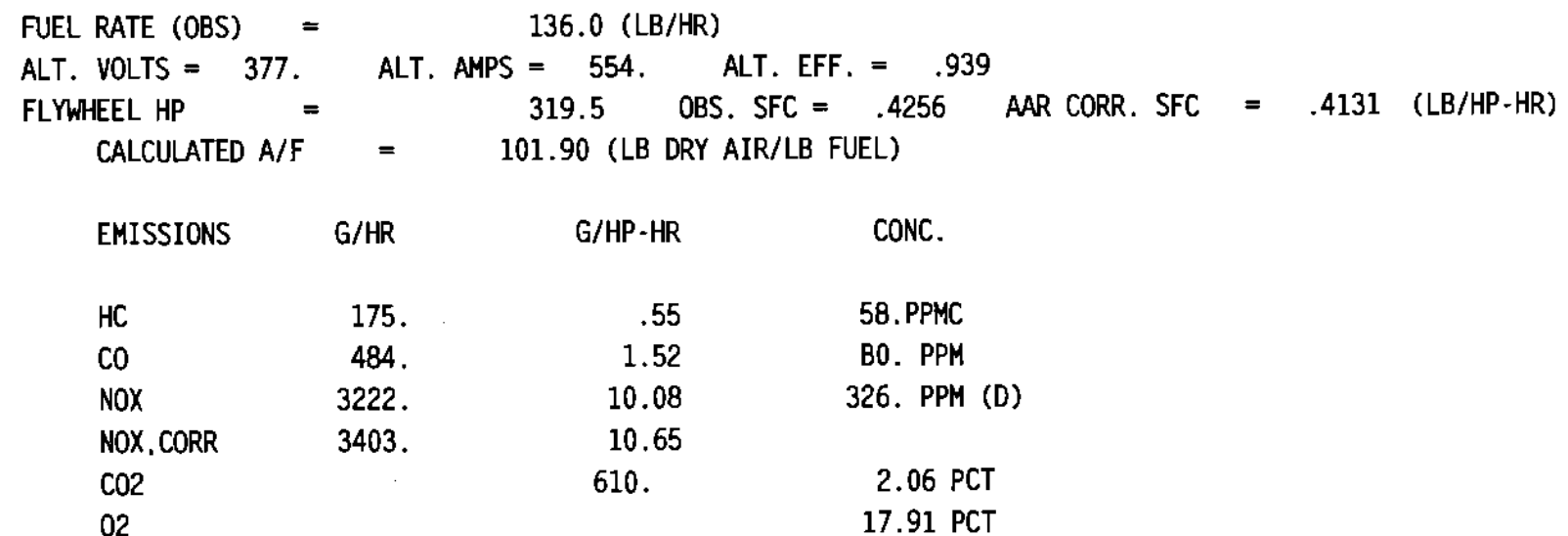




\begin{tabular}{|c|c|c|c|}
\hline LOCOMOTIVE MODEL & EMD GP38 & TEST LOCATION & SWRI SA,TX \\
\hline LOCOMOTIVE UNIT \# & $\operatorname{cs} x \# 2629$ & COMPUTER PROGRAM & EPA.FOR \\
\hline THROTTLE NOTCH & Notch 3 & TEST CONFIGURATION & $\mathrm{B} 20$ \\
\hline TEST DATE & $10 / 26 / 0$ & ENGINE MODEL & EMD $16-645 E 3$ \\
\hline TEST NUMBER & $5 / 10$ & & \\
\hline SWRI FUEL CODE & $E M-3004-F$ & FUEL H/C RATIO & 1.81 \\
\hline BAROMETER & 29.14 (in. $\mathrm{Hg}$ ) & ENGINE INTAKE AIR & $81 .\left(^{\circ} \mathrm{F}\right)$ \\
\hline DRY BULB TEMP & B3. $\left({ }^{\circ} \mathrm{F}\right)$ & WET BULB TEMP & 71. $\left({ }^{\circ} \mathrm{F}\right)$ \\
\hline ABS HUMIDITY & 95.21 (GR/LB) & RELATIVE HUMIDITY & 55. (y) \\
\hline
\end{tabular}

ENGINE PERFORMANCE SUMMARY:

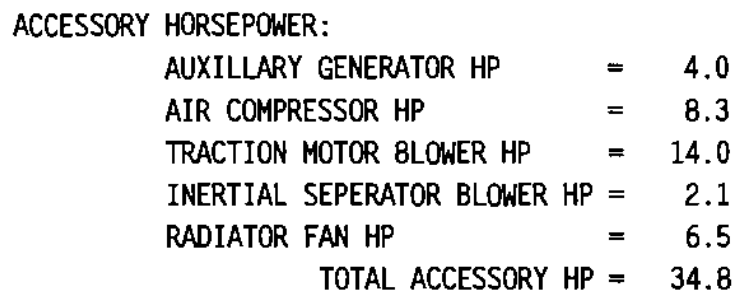




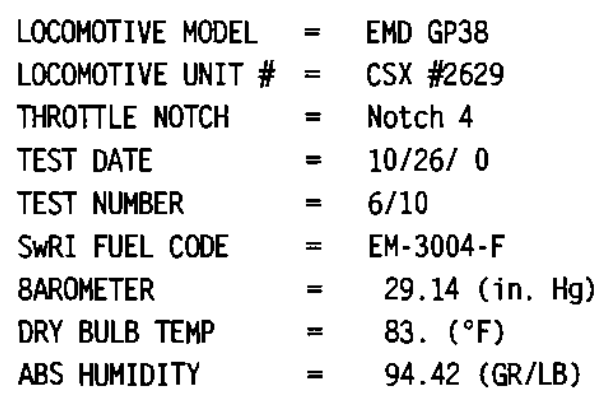

ENGINE PERFORMANCE SUMMARY:

ACCESSORY HORSEPOWER:

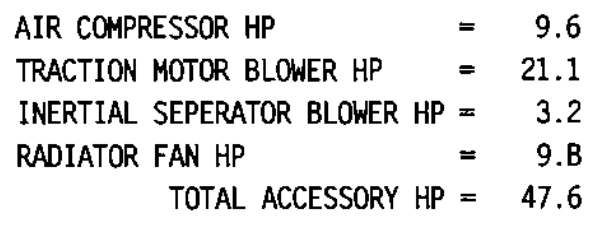

TOTAL ACCESSORY HP $=47.6$ \\ AUXILLARY GENERATOR HP $=\mathbf{4 . 0}$}

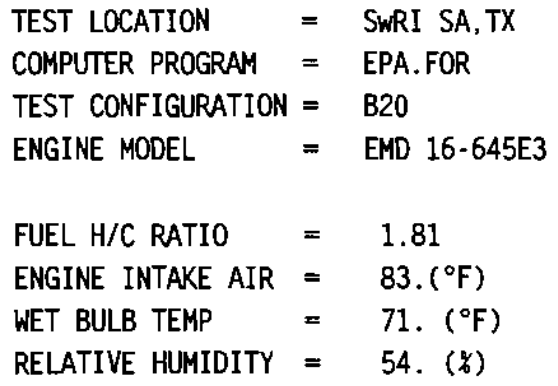

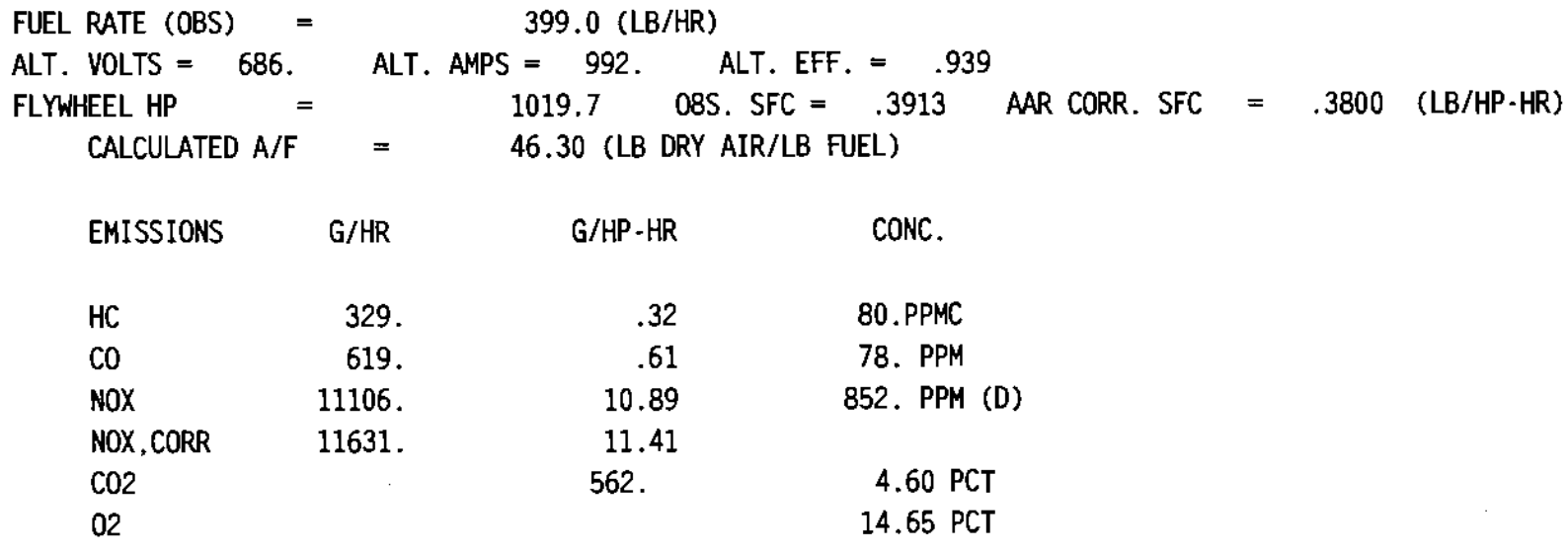

CORRECTION FACTOR SUMMARY:

$\begin{array}{lllll}\text { EPA NOX CF }=1.0473 & \text { NOX-KH } & =1.0387 & \text { NOX-KT } & =1.0000 \\ \text { WET EXH HC CF }=.9557 & \text { AIR TEMP CF }=.9896 & \text { BAROM CF } & =1.0024 \\ \text { FUEL TEMP CF }=.9840 & \text { FUEL S.G. CF }=.9832 & \text { FUEL HHV CF } & =.9789\end{array}$

PARTICULATE INFORMATION

FILTER PAIR I.D. NUMBER

$$
\begin{gathered}
6727.0-12 \\
8.985 \\
37.873 \\
.237 \\
5.844 \\
1.387 \\
348 . \\
.342
\end{gathered}
$$


LOCOMOTIVE EMISSIONS SWRI PROJECT NO. 08-2999-001

$\begin{array}{llll}\text { LOCOMOTIVE MODEL } & =\text { EMD GP38 } & \text { TEST LOCATION } & =\text { SWRI SA, TX } \\ \text { LOCOMOTIVE UNIT \# } & =\text { CSX \#2629 } & \text { COMPUTER PROGRAM } & =\text { EPA.FOR } \\ \text { THROTTLE NOTCH } & =\text { Notch } 5 & \text { TEST CONFIGURATION } & \text { B20 } \\ \text { TEST DATE } & =10 / 26 / 0 & \text { ENGINE MODEL } & =\text { EMD } 16-645 E 3 \\ \text { TEST NUMBER } & =7 / 10 & & \\ \text { SWRI FUEL CODE } & =\text { EM-3004-F } & \text { FUEL H/C RATIO } & =1.81 \\ \text { BAROMETER } & =29.13(\mathrm{in} . \mathrm{Hg}) & \text { ENGINE INTAKE AIR } & =81 .\left({ }^{\circ} \mathrm{F}\right) \\ \text { DRY BULB TEMP } & =83 .\left({ }^{\circ} \mathrm{F}\right) & \text { WET BULB TEMP } & =71 .\left({ }^{\circ} \mathrm{F}\right) \\ \text { ABS HUMIDITY } & =95.54(\mathrm{GR} / \mathrm{LB}) & \text { RELATIVE HUMIDITY } & =55 .(\mathrm{\gamma})\end{array}$

ENGINE PERFORMANCE SUMMARY:

ACCESSORY HORSEPOWER:

$\begin{array}{rrr}\text { AUXILLARY GENERATOR HP } & =4.0 \\ \text { AIR COMPRESSOR HP } & =10.8 \\ \text { TRACTION MOTOR BLOWER HP } & 30.1 \\ \text { INERTIAL SEPERATOR BLOWER HP } & =4.5 \\ \text { RADIATOR FAN HP } & =27.8 \\ \text { TOTAL ACCESSORY HP } & =77.2\end{array}$

FUEL RATE (OBS) $=500.0(\mathrm{LB} / \mathrm{HR})$

ALT. VOLTS $=760 . \quad$ ALT. AMPS $=1090 . \quad$ ALT. EFF. $=.939$

FLYWHEEL HP $=1260.6 \quad$ OBS. SFC $=.3966$ AAR CORR. SFC $=.3855$ (LB/HP-HR)

CALCULATED A/F $=42.38$ (LB DRY AIR/LB FUEL)

EMISSIONS G/HR G/HP-HR CONC.

$\begin{array}{lrrr}\text { HC } & 461 . & .37 & 98 . \text { PPMC } \\ \text { CO } & 1132 . & .90 & 125 . \text { PPM } \\ \text { NOX } & 14652 . & 11.62 & 982 . \text { PPM (D) } \\ \text { NOX.CORR } & 15385 . & 12.20 & \\ \text { C02 } & & 570 . & 5.03 \text { PCT } \\ 02 & & 13.90 \text { PCT }\end{array}$

CORRECTION FACTOR SUMMARY:

$\begin{array}{lllll}\text { EPA NOX CF }=1.0501 & \text { NOX-KH } & =1.0410 & \text { NOX-KT } & =1.0000 \\ \text { WET EXH HC CF }=.9521 & \text { AIR TEMP CF }=.9905 & \text { BAROM CF } & =1.0023 \\ \text { FUEL TEMP CF }=.9850 & \text { FUEL S.G. CF }=.9832 & \text { FUEL HHV CF } & =.9789\end{array}$

PARTICULATE INFORMATION

FILTER PAIR I.D. NUMBER

$672 \mathrm{~B} .0-13$

FILTER PAIR WEIGHT GAIN (mg)

8.007

37.274

DILUTE PM CONCENTRATION (mg/scf) $\quad .215$

CO2-BASED DILUTION FACTOR

6.601

1.418

PARTICULATE CONCENTRATION IN RAW EXHAUST $(\mathrm{mg} / \mathrm{sCf})$
PARTICULATE MASS EMISSION RATE $(\mathrm{g} / \mathrm{hr})$

BRAKE-SPECIFIC PARTICULATE EMISSION RATE $(\mathrm{g} / \mathrm{hp}-\mathrm{hr}) \quad .325$ 
LOCOMOTIVE EMISSIONS SWRI PROJECT NO. 08-2999.001

$\begin{array}{ll}\text { LOCOMOTIVE MODEL } & =\text { EMD GP38 } \\ \text { LOCOMOTIVE UNIT \# } & =\text { CSX } \# 2629 \\ \text { THROTTLE NOTCH } & =\text { Notch } 6 \\ \text { TEST DATE } & =10 / 26 / 0 \\ \text { TEST NUMBER } & =8 / 10 \\ \text { SWRI FUEL CODE } & =\text { EM-3004-F } \\ \text { BAROMETER } & =29.13(\mathrm{in} . \mathrm{Hg}) \\ \text { DRY BULB TEMP } & =82 .\left({ }^{\circ} \mathrm{F}\right) \\ \text { ABS HUMIDITY } & =94.28(\mathrm{GR} / \mathrm{LB})\end{array}$

$\begin{array}{ll}\text { TEST LOCATION } & =\text { SWRI SA,TX } \\ \text { COMPUTER PROGRAM } & =\text { EPA.FOR } \\ \text { TEST CONFIGURATION } & =\text { B20 } \\ \text { ENGINE MODEL } & =\text { EMD } 16 \cdot 645 E 3 \\ & \\ \text { FUEL H/C RATIO } & =1.81 \\ \text { ENGINE INTAKE AIR } & =84 .\left(^{\circ} \mathrm{F}\right) \\ \text { WET BULB TEMP } & =70 .\left({ }^{\circ} \mathrm{F}\right) \\ \text { RELATIVE HUMIDITY } & =55 .(\%)\end{array}$

ENGINE PERFORMANCE SUMMARY:

ACCESSORY HORSEPOWER:

$\begin{array}{rrr}\text { AUXILLARY GENERATOR HP } & = & 4.0 \\ \text { AIR COMPRESSOR HP } & =12.2 \\ \text { TRACTION MOTOR BLOWER HP } & =43.6 \\ \text { INERTIAL SEPERATOR BLOWER HP } & =6.5 \\ \text { RADIATOR FAN HP } & =40.3 \\ \text { TOTAL ACCESSORY HP } & =106.7\end{array}$

FUEL RATE (OBS) = $622.0(\mathrm{LB} / \mathrm{HR})$

ALT. VOLTS $=$ B38. ALT. AMPS $=1199 . \quad$ ALT. EFF. $=.939$

FLYWHELL HP $=1542.1 \quad$ OBS. SFC $=.4034 \quad$ AAR CORR. SFC $=.3915$ (LB/HP-HR)

CALCULATED A/F $=37.28$ (LB DRY AIR/LB FUEL)

EMISSIONS G/HR G/HP.HR CONC.

$\begin{array}{lrrr}\text { HC } & 596 . & .39 & 115 . \text { PPMC } \\ \text { CO } & 3822 . & 2.48 & 386 . \text { PPM } \\ \text { NOX } & 18229 . & 11.82 & 1119 . \text { PPM (D) } \\ \text { NOX, CORR } & 19084 . & 12.38 & \\ \text { C02 } & & 577 . & 5.71 \text { PCT } \\ \text { O2 } & & & 13.15 \text { PCT }\end{array}$

CORRECTION FACTOR SUMMARY:

$\begin{array}{lllll}\text { EPA NOX CF }=1.0469 & \text { NOX-KH } & =1.0384 & \text { NOX-KT } & =1.0000 \\ \text { WET EXH HC CF= } & .9465 & \text { AIR TEMP CF }=.9892 & \text { BAROM CF } & =1.0023 \\ \text { FUEL TEMP CF }=.9845 & \text { FUEL S.G. CF }=.9832 & \text { FUEL HHV CF } & =.9789\end{array}$

PARTICULATE INFORMATION

FILTER PAIR I.D. NUMBER

$6729.0-14$

FILTER PAIR WEIGHT GAIN $(\mathrm{mg})$

8.794

SAMPLE VOLUME (SCf)

37.002

DILUTE PM CONCENTRATION (mg/scf)

.238

7.540

1.792

PARTICULATE CONCENTRATION IN RAW EXHAUST (mg/scf)

PARTICULATE MASS EMISSION RATE $(\mathrm{g} / \mathrm{hr})$

568.

BRAKE-SPECIFIC PARTICULATE EMISSION RATE $(\mathrm{g} / \mathrm{hp}-\mathrm{hr})$ 


$\begin{array}{ll}\text { LOCOMOTIVE MODEL } & =\text { EMD GP38 } \\ \text { LOCOMOTIVE UNIT \# } & =\text { CSX } \# 2629 \\ \text { THROTTLE NOTCH } & =\text { Notch } 7 \\ \text { TEST DATE } & =10 / 26 / 0 \\ \text { TEST NUMBER } & =9 / 10 \\ \text { SWRI FUEL CODE } & =\text { EM-3004-F } \\ \text { BAROMETER } & =29.13(\mathrm{in} . \mathrm{Hg}) \\ \text { DRY BULB TEMP } & =83 .\left({ }^{\circ} \mathrm{F}\right) \\ \text { ABS HUMIDITY } & =97.20(\mathrm{GR} / \mathrm{LB})\end{array}$

ENGINE PERFORMANCE SUMMARY:

ACCESSORY HORSEPOWER:

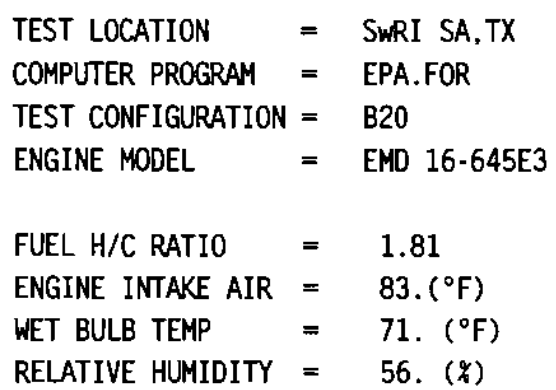

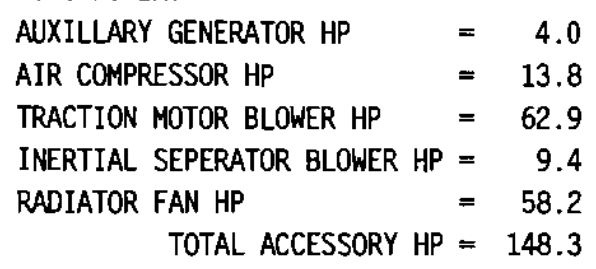

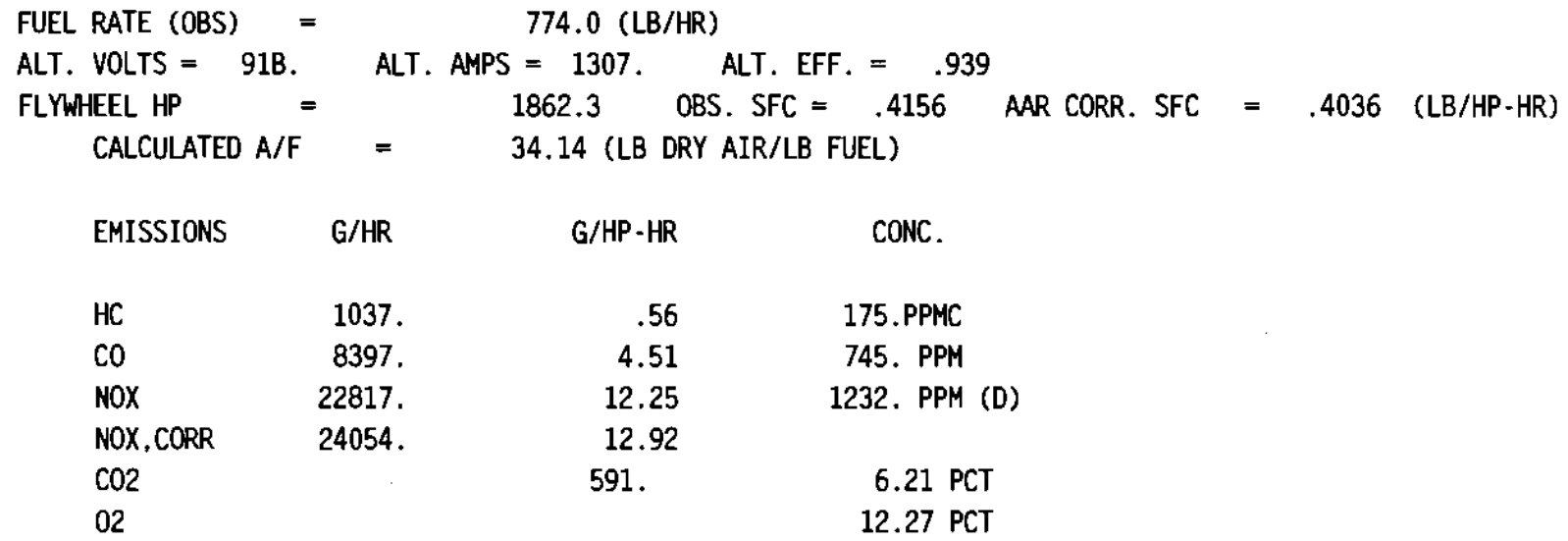

CORRECTION FACTOR SUMMARY:

$\begin{array}{lllll}\text { EPA NOX CF }=1.0542 & \text { NOX }- \text { KH } & =1.0443 & \text { NOX-KT } & =1.0000 \\ \text { WET EXH HC CF }=.9421 & \text { AIR TEMP CF }=.9896 & \text { BAROM CF } & =1.0023 \\ \text { FUEL TEMP CF }=.9855 & \text { FUEL S.G. CF }=.9832 & \text { FUEL HHV CF }=.9789\end{array}$

PARTICULATE INFORMATION

FILTER PAIR I.D. NUMBER

$6730.0-15$

FILTER PAIR WEIGHT GAIN (mg)

12.061

35.951

SAMPLE VOLUME (scf)

.335

DILUTE PM CONCENTRATION (mg/sCf)

7.774

2.608

PARTICULATE CONCENTRATION IN RAW EXHAUST ( $\mathrm{mg} / \mathrm{scf}$ )

PARTICULATE MASS EMISSION RATE $(\mathrm{g} / \mathrm{hr})$

944.

BRAKE-SPECIFIC PARTICULATE EMISSION RATE $(g / h p-h r)$ 


$\begin{array}{ll}\text { LOCOMOTIVE MODEL } & =\text { EMD GP38 } \\ \text { LOCOMOTIVE UNIT. \# } & =\text { CSX } \# 2629 \\ \text { THROTTLE NOTCH } & =\text { Notch } 8 \\ \text { TEST DATE } & =10 / 26 / 0 \\ \text { TEST NUMBER } & =10 / 10 \\ \text { SWRI FUEL CODE } & =\text { EM-3004-F } \\ \text { BAROMETER } & =29.13(\mathrm{in} . \mathrm{Hg}) \\ \text { DRY BULB TEMP } & =84 .\left({ }^{\circ} \mathrm{F}\right) \\ \text { ABS HUMIDITY } & =96.48(\mathrm{GR} / \mathrm{LB})\end{array}$

ENGINE PERFORMANCE SUMMARY:

ACCESSORY HORSEPOWER:
AUXILLARY GENERATOR HP
AIR COMPRESSOR HP
$=4.0$
TRACTION MOTOR BLOWER HP $=82.8$
INERTIAL SEPERATOR BLOWER HP $=12.4$
RADIATOR FAN HP $\quad=76.6$
TOTAL ACCESSORY HP $=191.0$

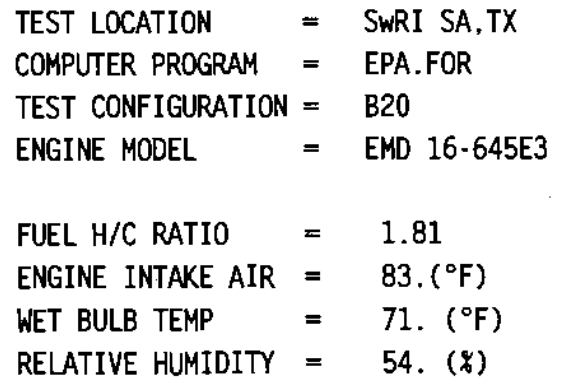

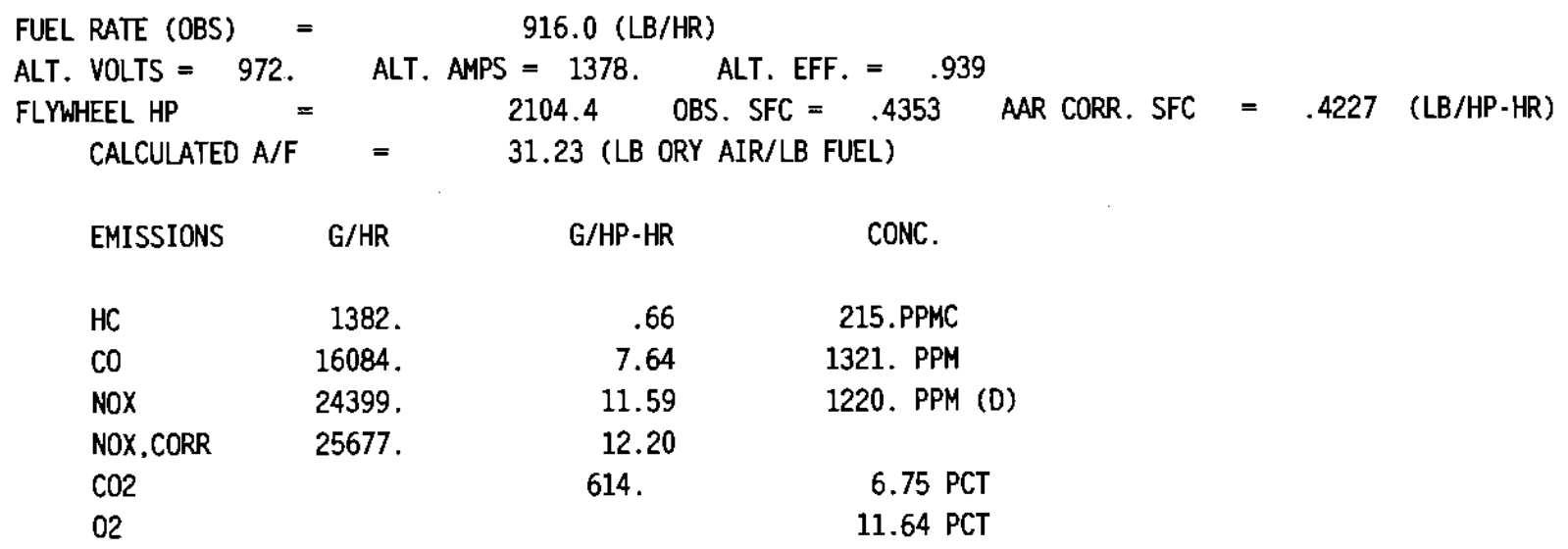

CORRECTION FACTOR SUMMARY:

$\begin{array}{lllll}\text { EPA NOX CF }=1.0524 & \text { NOX-KH } & =1.0429 & \text { NOX-KT } & =1.0000 \\ \text { WET EXH HC CF }=.9375 & \text { AIR TEMP CF }=.9896 & \text { BAROM CF } & =1.0023 \\ \text { FUEL TEMP CF }=.9850 & \text { FUEL S.G. CF }=.9832 & \text { FUEL HHV CF }=.9789\end{array}$

PARTICULATE INFORMATION

FILTER PAIR I.D. NUMBER

$6731.0-16$

FILTER PAIR WEIGHT GAIN (mg)

13.884

35.266

DILUTE PM CONCENTRATION (mg/scf)

.394

7.428

CO2-BASED DILUTION FACTOR

2.924

PARTICULATE CONCENTRATION IN RAW EXHAUST (mg/scf)

PARTICULATE MASS EMISSION RATE $(\mathrm{g} / \mathrm{hr}$ )

1149.

BRAKE-SPECIFIC PARTICULATE EMISSION RATE $(\mathrm{g} / \mathrm{hp}-\mathrm{hr})$ 


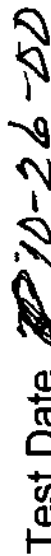

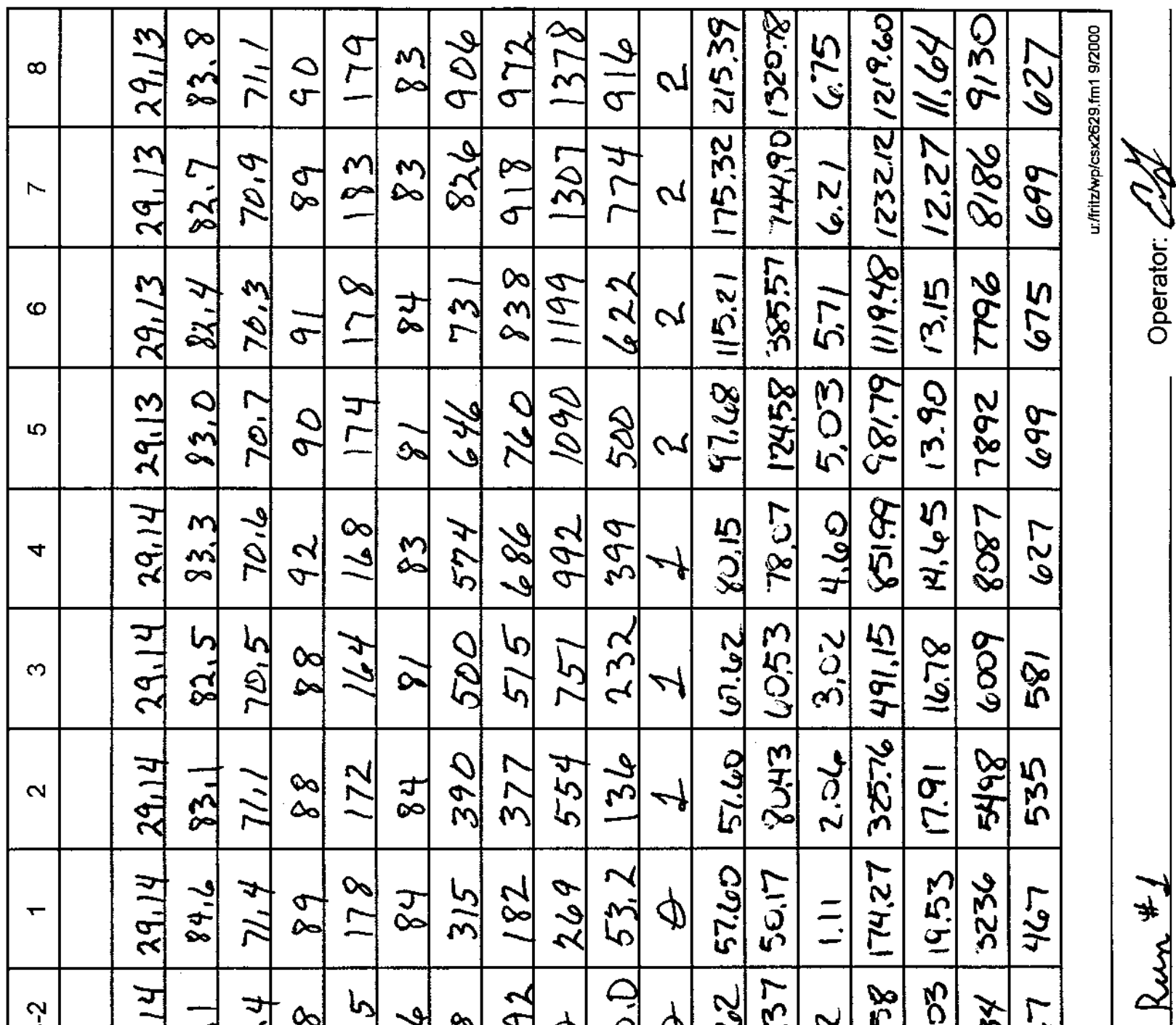

烍

กั

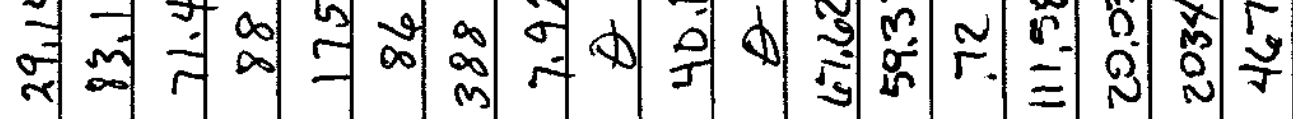

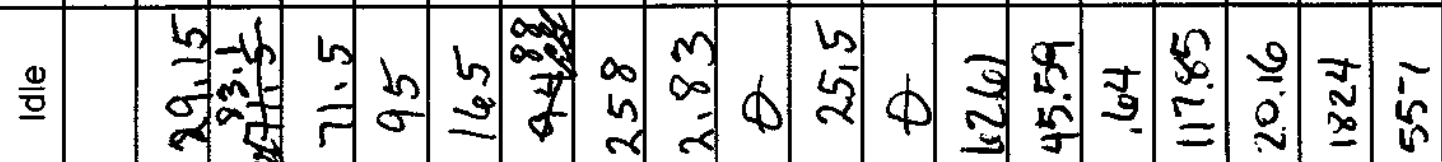

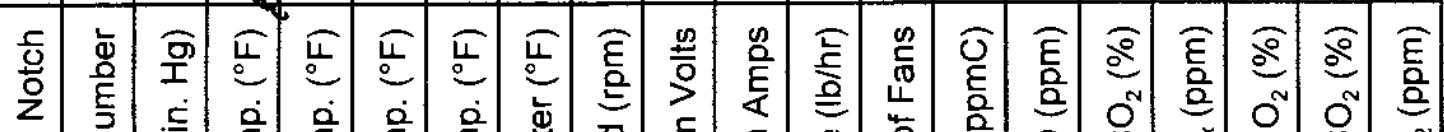

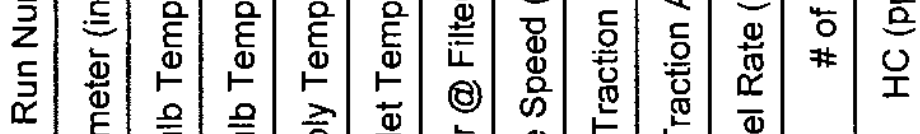

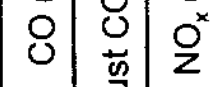

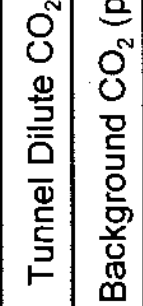

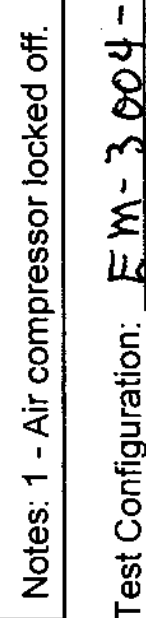




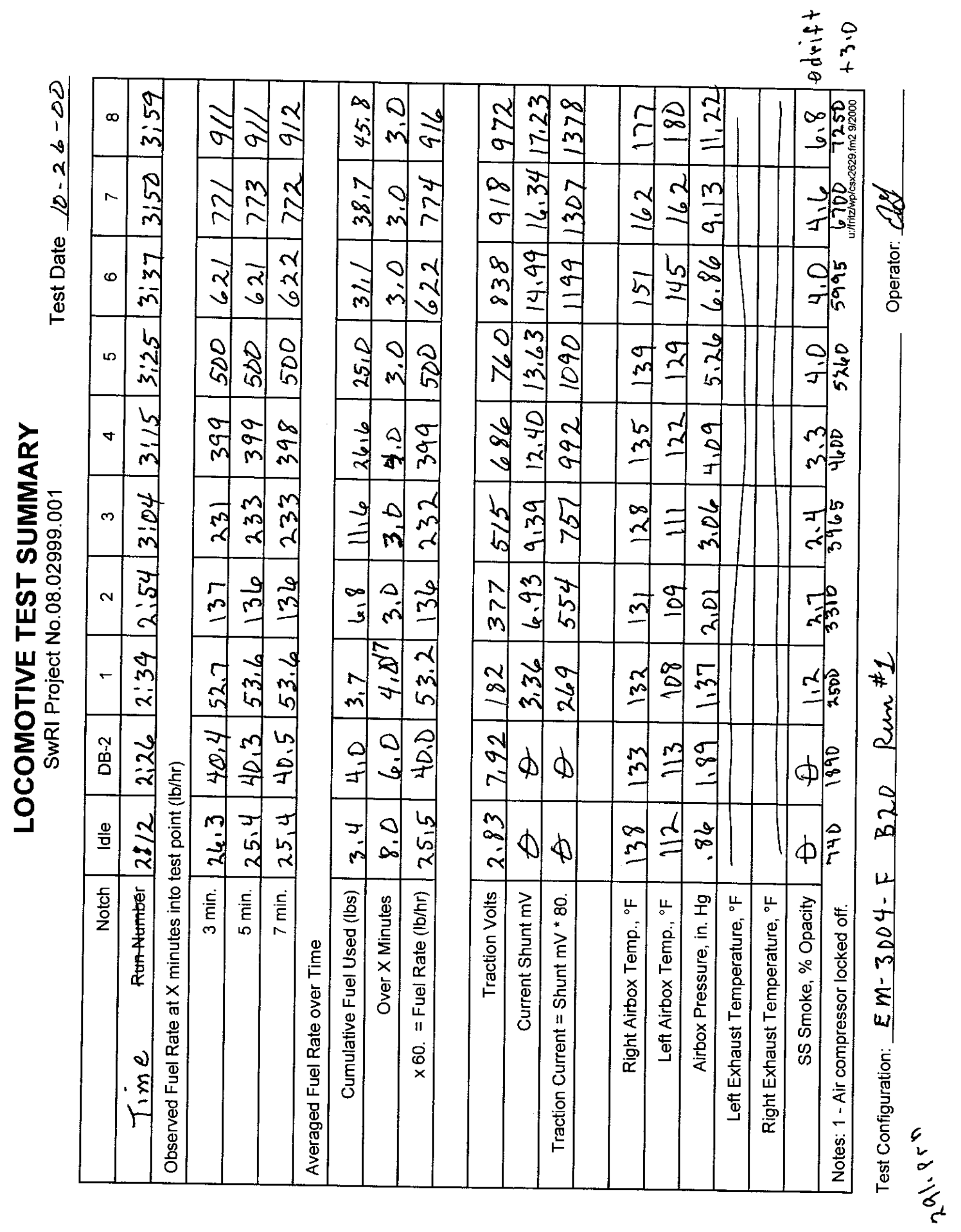




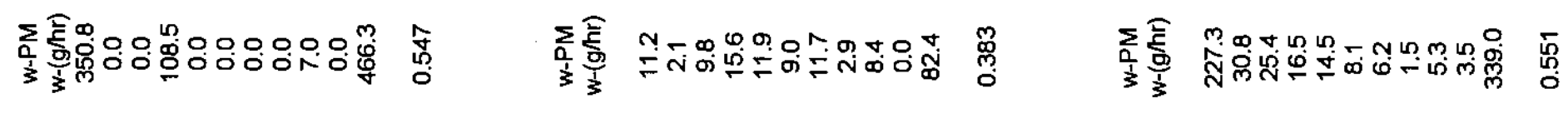

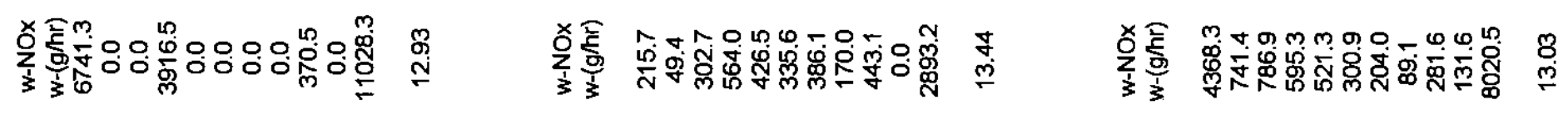

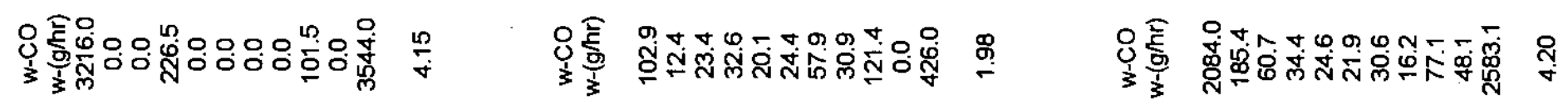

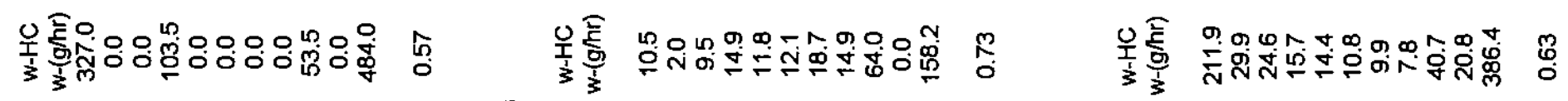

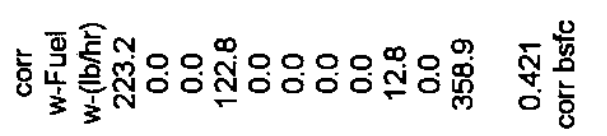

害

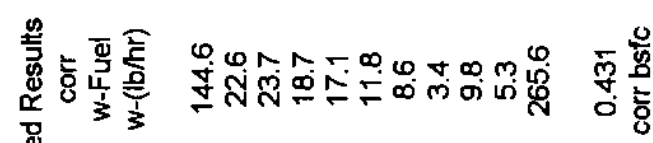

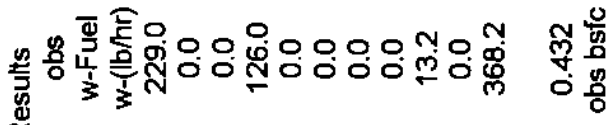

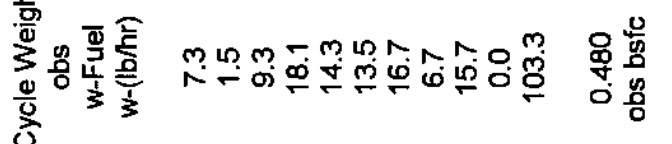

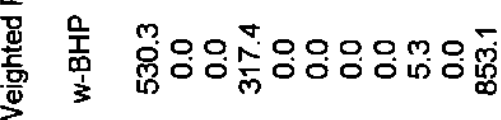

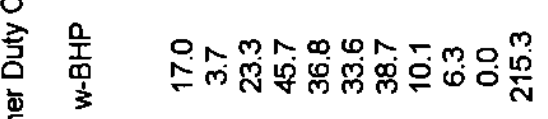

s

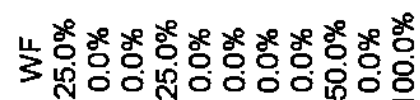

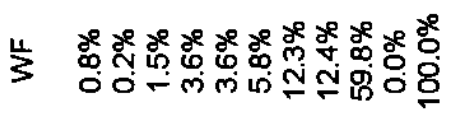

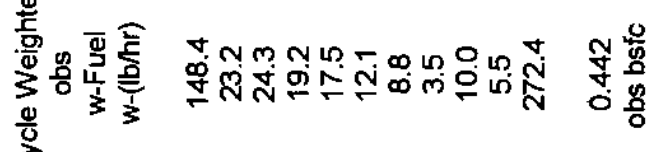

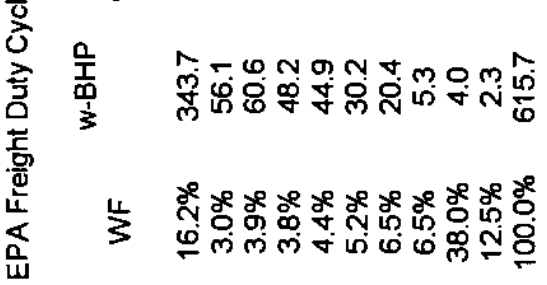

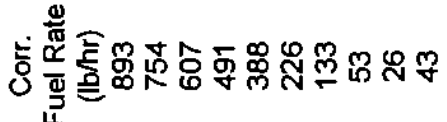

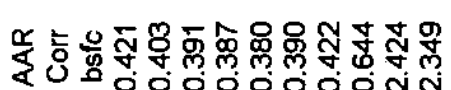

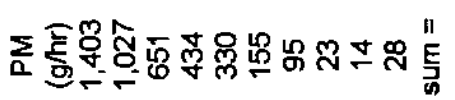

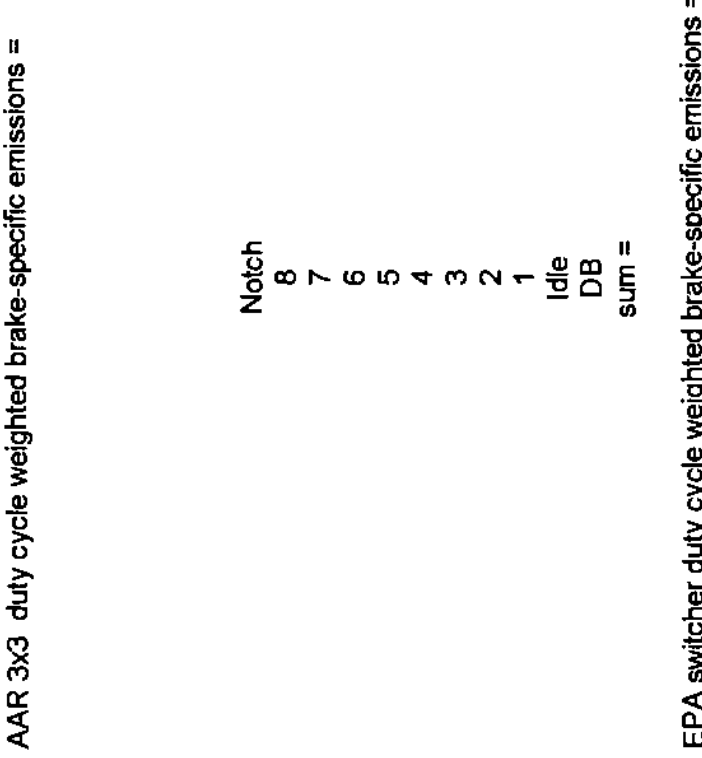

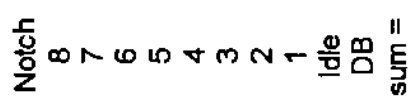

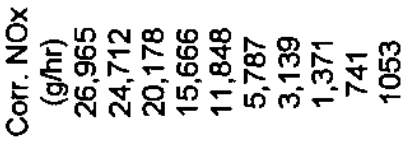

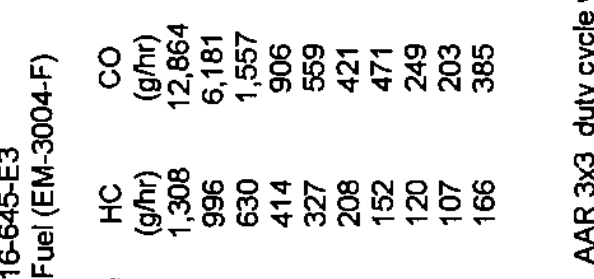

㽞

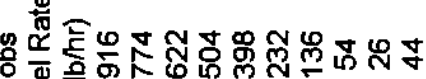

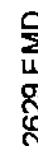

gु

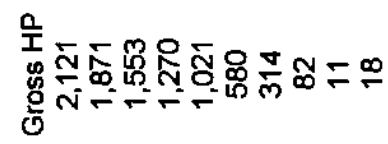

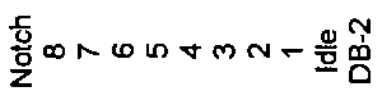


LOCOMOTIVE EMISSIONS SWRI PROJECT NO. 08-2999-001

$\begin{array}{ll}\text { LOCOMOTIVE MODEL } & =\text { EMD GP38 } \\ \text { LOCOMOTIVE UNIT \# } & =\text { CSX } \# 2629 \\ \text { THROTTLE NOTCH } & =\text { DB2 } \\ \text { TEST DATE } & =10 / 30 / 0 \\ \text { TEST NUMBER } & =2 / 10 \\ \text { SWRI FUEL CODE } & =\text { EM }-3004 \cdot \mathrm{F} \\ \text { BAROMETER } & =29.26(\mathrm{in} . \mathrm{Hg}) \\ \text { DRY BULB TEMP } & =76 .\left(^{\circ} \mathrm{F}\right) \\ \text { ABS HUMIDITY } & =112.16(\mathrm{GR} / \mathrm{LB})\end{array}$

$\begin{array}{ll}\text { TEST LOCATION } & =\text { SWRI SA,TX } \\ \text { COMPUTER PROGRAM } & =\text { EPA.FOR } \\ \text { TEST CONFIGURATION } & =\text { B20 } \\ \text { ENGINE MODEL } & =\text { EMD } 16-645 E 3 \\ \text { FUEL H/C RATIO } & =1.81 \\ \text { ENGINE INTAKE AIR } & =79 .\left(^{\circ} \mathrm{F}\right) \\ \text { WET BULB TEMP } & =72 .\left({ }^{\circ} \mathrm{F}\right) \\ \text { RELATIVE HUMIDITY } & =82 .(\mathrm{\%})\end{array}$

ENGINE PERFORMANCE SUMMARY:

ACCESSORY HORSEPOWER:

$\begin{array}{rlr}\text { AUXILLARY GENERATOR HP } & =4.0 \\ \text { AIR COMPRESSOR HP } & =6.5 \\ \text { TRACTION MOTOR BLOWER HP } & =6.7 \\ \text { INERTIAL SEPERATOR BLONER HP } & =1.0 \\ \text { RADIATOR FAN HP } & .0 \\ \text { TOTAL ACCESSORY HP } & =18.2\end{array}$

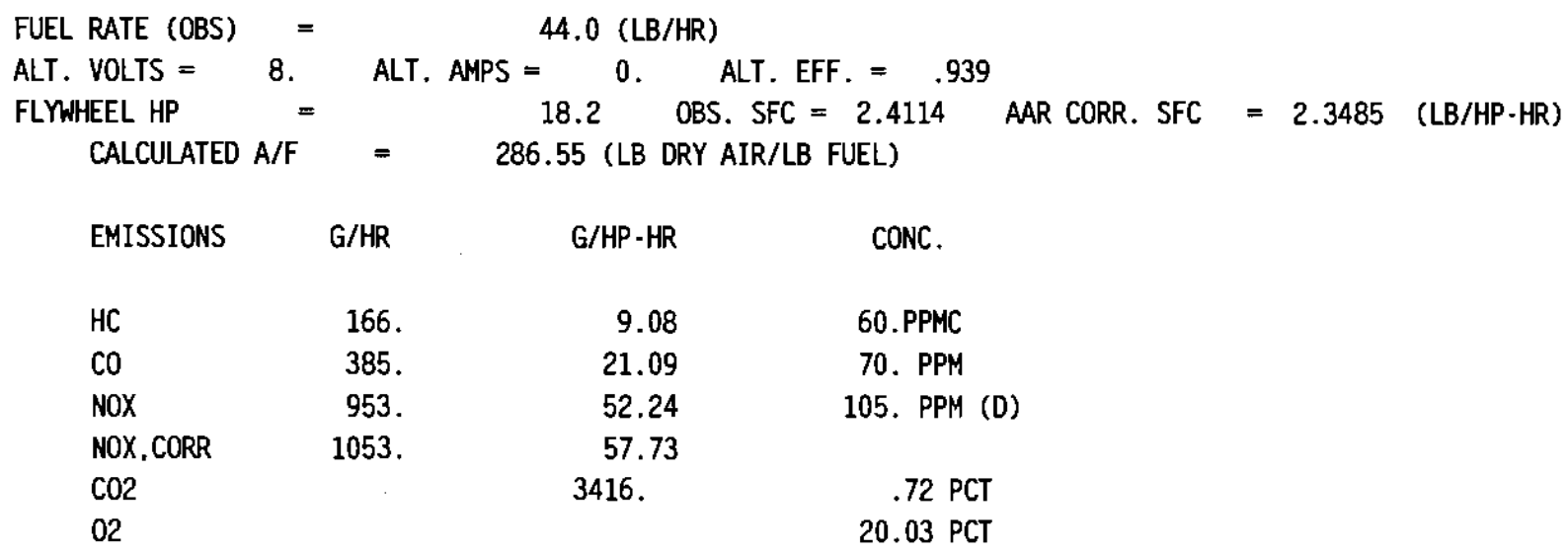

CORRECTION FACTOR SUMMARY:

$\begin{array}{lllll}\text { EPA NOX CF }=1.1050 & \text { NOX-KH } & =1.0856 & \text { NOX-KT } & =1.0000 \\ \text { WET EXH HC CF }=.9882 & \text { AIR TEMP CF }=.9914 & \text { BAROM CF } & =1.0035 \\ \text { FUEL TEMP CF }=.9820 & \text { FUEL S.G. CF }=.9832 & \text { FUEL HHV CF }=.9789\end{array}$

PARTICULATE INFORMATION

FILTER PAIR I.D. NUMBER

FILTER PAIR WEIGHT GAIN (mg)

$6753.0-38$

1.451

SAMPLE VOLUME (sCf)

38.522

DILUTE PM CONCENTRATION (mg/scf)

.038

CO2-BASED DILUTION FACTOR

4.394

.166

PARTICULATE CONCENTRATION IN RAW EXHAUST (mg/scf)

PARTICULATE MASS EMISSION RATE $(\mathrm{g} / \mathrm{hr})$

28.

BRAKE-SPECIFIC PARTICULATE EMISSION RATE $(g / h p-h r)$

1.527 


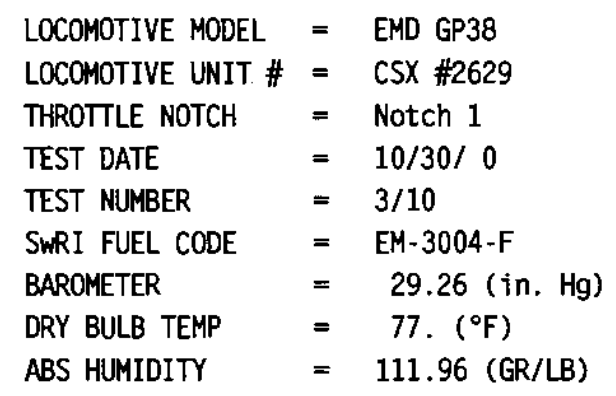

ENGINE PERFORMANCE SUMMARY:

\section{ACCESSORY HORSEPOWER:}

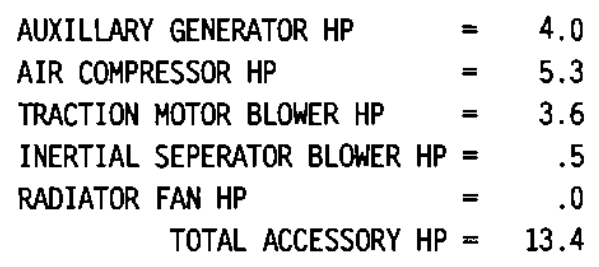

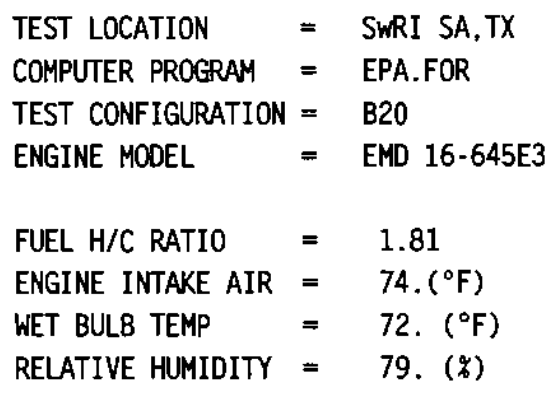




$\begin{array}{ll}\text { LOCOMOTIVE MODEL } & =\text { EMD GP38 } \\ \text { LOCOMOTIVE UNIT \# } & =\text { CSX } \# 2629 \\ \text { THROTTLE NOTCH } & =\text { Notch } 3 \\ \text { TEST DATE } & =10 / 30 / 0 \\ \text { TEST NUMBER } & =5 / 10 \\ \text { SWRI FUEL CODE } & =\text { EM-3004-F } \\ \text { BAROMETER } & =29.26(\text { in. Hg) } \\ \text { DRY BULB TEMP } & \left.=7 \mathrm{~B} .{ }^{\circ} \mathrm{F}\right) \\ \text { ABS HUMIDITY } & =110.56(\mathrm{GR} / \mathrm{LB})\end{array}$

ENGINE PERFORMANCE SUMMARY;

ACCESSORY HORSEPOWER:

$\begin{array}{rrr}\text { AUXILLARY GENERATOR HP } & = & 4.0 \\ \text { AIR COMPRESSOR HP } & = & 8.4 \\ \text { TRACTION MOTOR BLOWER HP } & =14.2 \\ \text { INERTIAL SEPERATOR BLOWER HP } & = & 2.1 \\ \text { RADIATOR FAN HP } & =6.6 \\ \text { TOTAL ACCESSORY HP } & =35.3\end{array}$

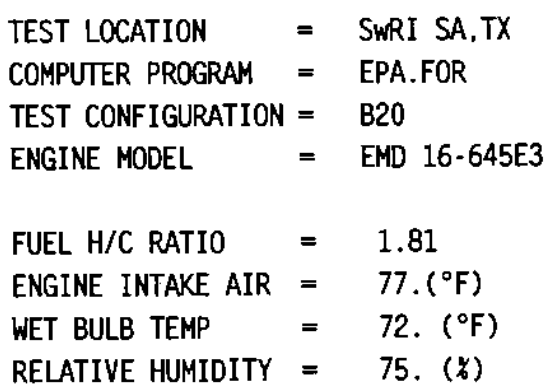

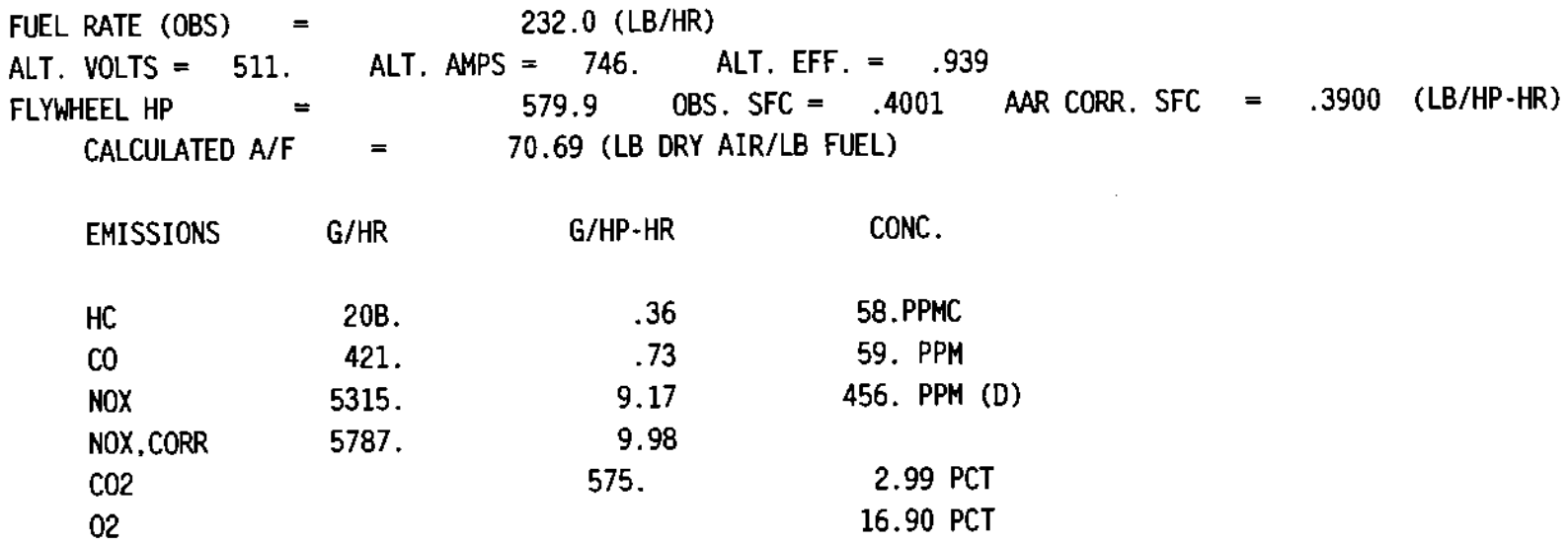

CORRECTION FACTOR SUMMARY:

$\begin{array}{lllll}\text { EPA NOX CF }=1.0887 & \text { NOX-KH } & =1.0724 & \text { NOX-KT } & =1.0000 \\ \text { WET EXH HC CF }=.9685 & \text { AIR TEMP CF }=.9923 & \text { BAROM CF } & =1.0035 \\ \text { FUEL TEMP CF }=.9835 & \text { FUEL S.G. CF }=.9832 & \text { FUEL HHV CF } & =.9789\end{array}$

PARTICULATE INFORMATION

FILTER PAIR I.D. NUMBER

$6766.0-51$

FILTER PAIR WEIGHT GAIN (mg)

5.299

38.216

SAMPLE VOLUME (sCf)

.139

5.040

CO2-BASED DILUTION FACTOR

.699

PARTICULATE CONCENTRATION IN RAW EXHAUST ( $\mathrm{mg} / \mathrm{scf}$ )

155.

BRAKE-SPECIFIC PARTICULATE EMISSION RATE $(\mathrm{g} / \mathrm{hp}-\mathrm{hr})$ 


$\begin{array}{lll}\text { LOCOMOTIVE MODEL } & =\text { EMD GP38 } \\ \text { LOCOMOTIVE UNIT \# } & =\text { CSX \#2629 } \\ \text { THROTTLE NOTCH } & =\text { Notch } 4 \\ \text { TEST DATE } & =10 / 30 / 0 \\ \text { TEST NUMBER } & =6 / 10 \\ \text { SWRI FUEL CODE } & =\text { EM-3004-F } \\ \text { BAROMETER } & =29.26(\mathrm{in} . \mathrm{Hg}) \\ \text { DRY BULB TEMP } & =79 .\left(^{\circ} \mathrm{F}\right) \\ \text { ABS HUMIDITY } & =108.84(\mathrm{GR} / \mathrm{LB})\end{array}$

ENGINE PERFORMANCE SUMMARY:

ACCESSORY HORSEPOWER:

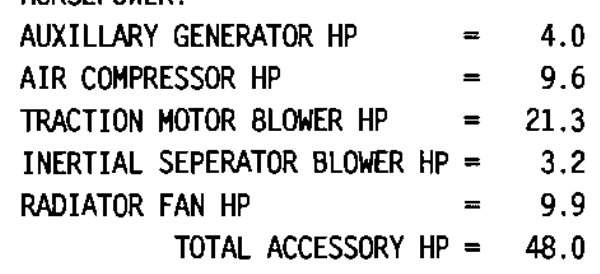

$\begin{array}{rlr}\text { AUXILLARY GENERATOR HP } & =4.0 \\ \text { AIR COMPRESSOR HP } & =9.6 \\ \text { TRACTION MOTOR BLOWER HP } & =21.3 \\ \text { INERTIAL SEPERATOR BLOWER HP } & =3.2 \\ \text { RADIATOR FAN HP } & 9.9 \\ \text { TOTAL ACCESSORY HP } & =48.0\end{array}$

TOTAL ACCESSORY HP $=48.0$

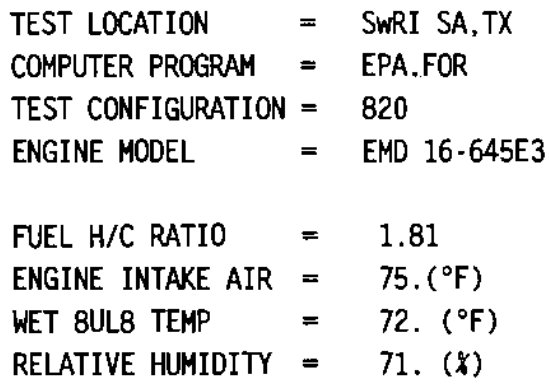

FUEL RATE (OBS) = $398.0(\mathrm{LB} / \mathrm{HR})$

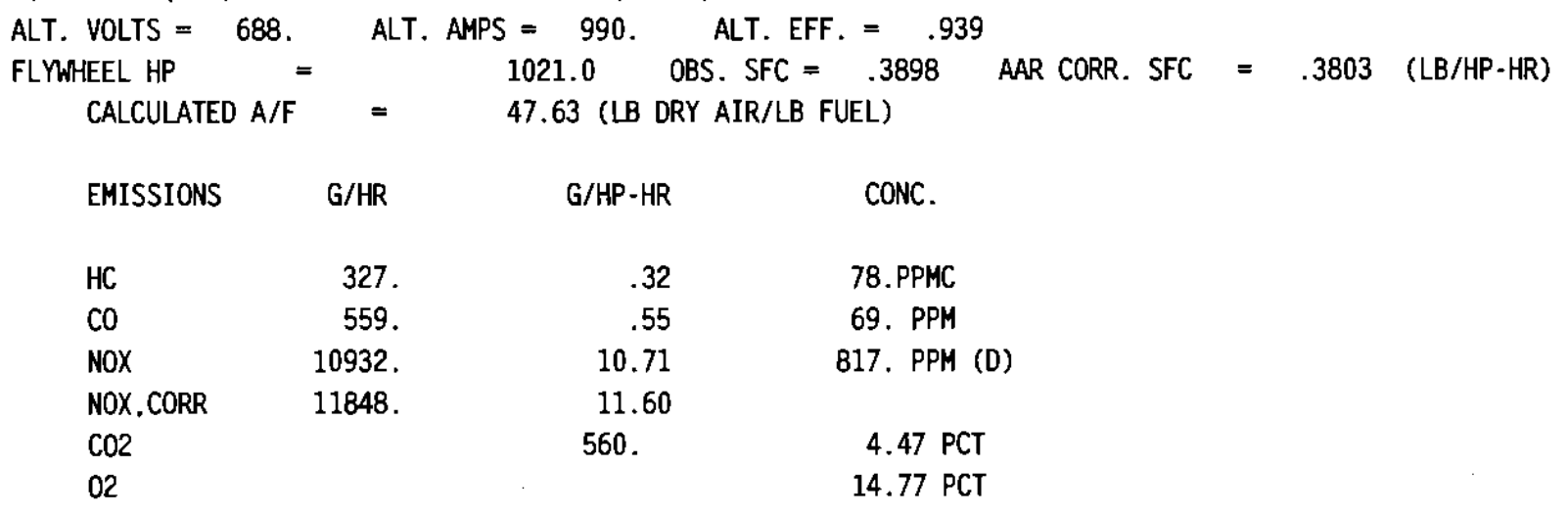

CORRECTION FACTOR SUMMARY:

$\begin{array}{lllll}\text { EPA NOX CF }=1.0838 & \text { NOX-KH } & =1.0684 & \text { NOX-KT } & =1.0000 \\ \text { WET EXH HC CF }=.9562 & \text { AIR TEMP CF }=.9932 & \text { BAROM CF } & =1.0035 \\ \text { FUEL TEMP CF }=.9840 & \text { FUEL S.G. CF }=.9832 & \text { FUEL HHV CF } & =.9789\end{array}$

PARTICULATE INFORMATION

FILTER PAIR I.D. NUMBER

$6767.0-52$

7.792

38.055

SAMPLE VOLUME (scf)

DILUTE PM CONCENTRATION (mg/scf)

.205

6.263

1.282

330 .

.324 


$\begin{array}{ll}\text { LOCOMOTIVE MODEL } & =\text { EMD GP38 } \\ \text { LOCOMOTIVE UNIT \# } & =\text { CSX } \# 2629 \\ \text { THROTTLE NOTCH } & =\text { Notch } 5 \\ \text { TEST DATE } & =10 / 30 / 0 \\ \text { TEST NUMBER } & =7 / 10 \\ \text { SWRI FUEL CODE } & =\text { EM-3004-F } \\ \text { 8AROMETER } & =29.26(\mathrm{in} . \mathrm{Hg}) \\ \text { DRY BULB TEMP } & =79 .\left({ }^{\circ} \mathrm{F}\right) \\ \text { ABS HUMIDITY } & =108.25(\mathrm{GR} / \mathrm{LB})\end{array}$

ENGINE PERFORMANCE SUMMARY:

ACCESSORY HORSEPOWER:

$\begin{array}{ll}\text { TEST LOCATION } & =\text { SWRI SA,TX } \\ \text { COMPUTER PROGRAM } & =\text { EPA.FOR } \\ \text { TEST CONFIGURATION } & =\text { B20 } \\ \text { ENGINE MOOEL } & =\text { EMD } 16-645 E 3 \\ \text { FUEL H/C RATIO } & =1.81 \\ \text { ENGINE INTAKE AIR } & =77 .\left(^{\circ} \mathrm{F}\right) \\ \text { WET BULB TEMP } & =72 .\left(^{\circ} \mathrm{F}\right) \\ \text { RELATIVE HUMIDITY } & =71 .(\%)\end{array}$

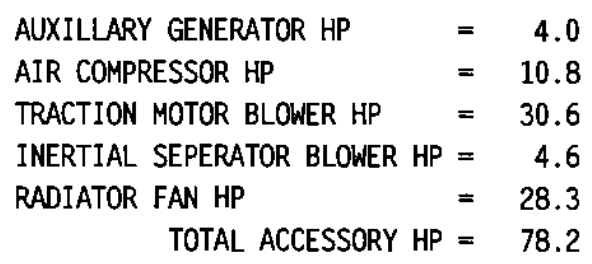

CORRECTION FACTOR SUMMARY:

$\begin{array}{lllll}\text { EPA NOX CF }=1.0822 & \text { NOX-KH } & =1.0671 & \text { NOX-KT } & =1.0000 \\ \text { WET EXH HC CF }=.9512 & \text { AIR TEMP CF }=.9923 & \text { BAROM CF } & =1.0035 \\ \text { FUEL TEMP CF }=.9840 & \text { FUEL S.G. CF }=.9832 & \text { FUEL HHV CF }=.9789\end{array}$

PARTICULATE INFORMATION

FILTER PAIR I.D. NUM8ER

$6768.0-53$

8.319

37.679

.221

6.804

1.502

434.

.342 


$\begin{array}{ll}\text { LOCOMOTIVE MODEL } & =\text { EMD GP38 } \\ \text { LOCOMOTIVE UNIT \# } & =\text { CSX \#2629 } \\ \text { THROTTLE NOTCH } & =\text { Notch } 6 \\ \text { TEST DATE } & =10 / 30 / 0 \\ \text { TEST NUMBER } & =8 / 10 \\ \text { SWRI FUEL CODE } & =\text { EM-3004-F } \\ \text { BAROMETER } & =29.26(\mathrm{in} . \mathrm{Hg}) \\ \text { DRY BULB TEMP } & =79 .\left(^{\circ} \mathrm{F}\right) \\ \text { ABS HUMIDITY } & =108.15(\mathrm{GR} / \mathrm{LB})\end{array}$

ENGINE PERFORMANCE SUMMARY:

ACCESSORY HORSEPOWER:

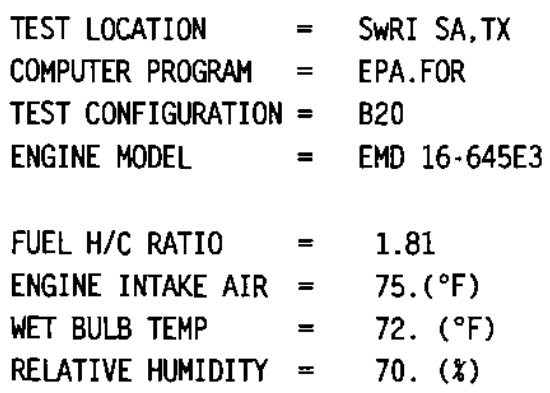

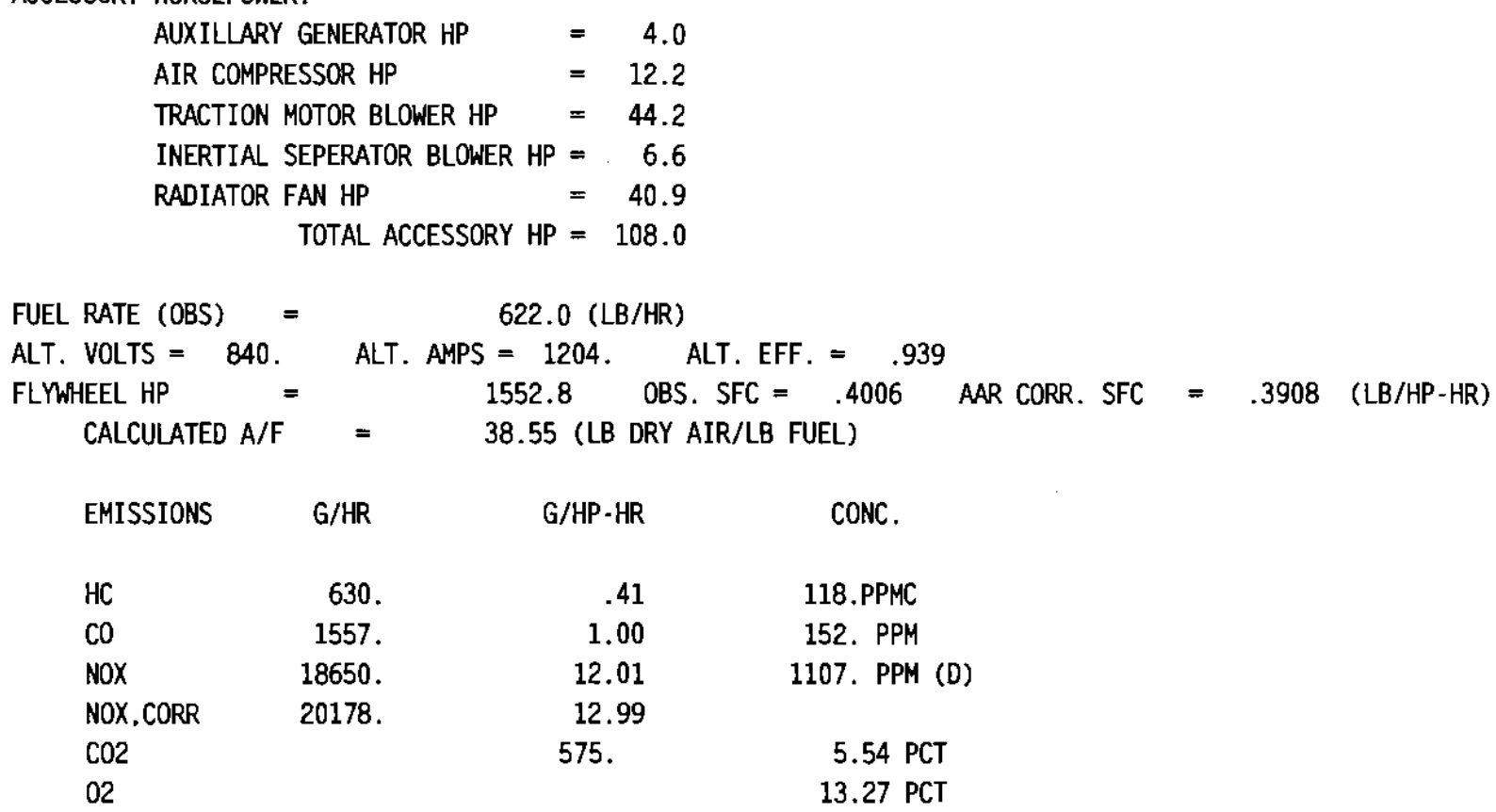

$\begin{array}{rlr}\text { AUXILLARY GENERATOR HP } & = & 4.0 \\ \text { AIR COMPRESSOR HP } & =12.2 \\ \text { TRACTION MOTOR BLOWER HP } & =44.2 \\ \text { INERTIAL SEPERATOR BLOWER HP } & =6.6 \\ \text { RADIATOR FAN HP } & 40.9 \\ \text { TOTAL ACCESSORY HP } & =108.0\end{array}$

CORRECTION FACTOR SUMMARY:

EPA NOX CF $=1.0819$ WET EXH HC CF $=.9474$ FUEL TEMP CF $=.9850$ NOX-KH $=1.0669$ AIR TEMP CF $=.9932$ FUEL S.G. CF $=.9832$

PARTICULATE INFORMATION

FILTER PAIR I.D. NUMBER

$6769.0-54$

9.607

37.365

.257

7.735

1.989

651.

.419 
LOCOMOTIVE EMISSIONS SWRI PROJECT NO. 08-2999-001

$\begin{array}{llll}\text { LOCOMOTIVE MODEL } & =\text { EMD GP38 } & \text { TEST LOCATION } & =\text { SWRI SA,TX } \\ \text { LOCOMOTIVE UNIT \# } & =\text { CSX } \# 2629 & \text { COMPUTER PROGRAM } & =\text { EPA.FOR } \\ \text { THROTTLE NOTCH } & =\text { Notch } 7 & \text { TEST CONFIGURATION } & \text { B20 } \\ \text { TEST DATE } & =10 / 30 / 0 & \text { ENGINE MODEL } & =\text { EMD } 16-645 E 3 \\ \text { TEST NUMBER } & =9 / 10 & & \\ \text { SWRI FUEL CODE } & =\text { EM-3004-F } & \text { FUEL H/C RATIO } & =1.81 \\ \text { BAROMETER } & =29.26(\mathrm{in} . \mathrm{Hg}) & \text { ENGINE INTAKF AIR } & =78 .\left({ }^{\circ} \mathrm{F}\right) \\ \text { DRY BULB TEMP } & =\mathrm{B} 0 .\left({ }^{\circ} \mathrm{F}\right) & \text { WET BULB TEMP } & =72 .\left({ }^{\circ} \mathrm{F}\right) \\ \text { ABS HUMIDITY } & =107.21(\mathrm{GR} / \mathrm{LB}) & \text { RELATIVE HUMIDITY } & =69 .(\%)\end{array}$

ENGINE PERFORMANCE SUMMARY:

ACCESSORY HORSEPOWER:

$\begin{array}{rrr}\text { AUXILLARY GENERATOR HP } & =4.0 \\ \text { AIR COMPRESSOR HP } & 13.8 \\ \text { TRACTION MOTOR BLOWER HP } & =63.5 \\ \text { INERTIAL SEPERATOR BLOWER HP } & =9.5 \\ \text { RADIATOR FAN HP } & 58.8 \\ \text { TOTAL ACCESSORY HP } & =149.6\end{array}$

FUEL RATE (OBS) = 774.0 (LB/HR)

ALT. VOLTS $=920 . \quad$ ALT. AMPS $=1310 . \quad$ ALT. EFF. $=.939$

FLYHEEL HP $=\quad 1871.3 \quad$ OBS. SFC $=.4136 \quad$ AAR CORR. SFC $=.4030$ (LB/HP-HR)

CALCULATED A/F $=34.26$ (LB DRY AIR/LB FUEL)

EMISSIONS G/HR G/HP-HR CONC.

$\begin{array}{lrrr}\text { HC } & 996 . & .53 & 168 . \text { PPMC } \\ \text { CO } & 6181 . & 3.30 & 547 . \text { PPM } \\ \text { NOX } & 22892 . & 12.23 & 1232 . \text { PPM (D) } \\ \text { NOX,CORR } & 24712 . & 13.21 & \\ \text { C02 } & & 590 . & 6.21 \text { PCT } \\ 02 & & & 12.40 \text { PCT }\end{array}$

CORRECTION FACTOR SUMMARY:

$\begin{array}{lllll}\text { EPA NOX CF }=1.0795 & \text { NOX-KH } & =1.0649 & \text { NOX-KT } & =1.0000 \\ \text { WET EXH HC CF }=.9418 & \text { AIR TEMP CF }=.9919 & \text { BAROM CF } & =1.0035 \\ \text { FUEL TEMP CF }=.9855 & \text { FUEL S.G. CF }=.9832 & \text { FUEL HHV CF } & =.9789\end{array}$

PARTICULATE INFORMATION

FILTER PAIR I.D. NUMBER

$6770.0 \cdot 55$

FILTER PAIR WEIGHT GAIN (mg)

13.191

36.611

SAMPLE VOLUME (sCf)

.360

DILUTE PM CONCENTRATION (mg/scf)

7.849

CO2-BASED DILUTION FACTOR

2.828

PARTICULATE MASS EMISSION RATE $(g / h r)$

1027.

BRAKE-SPECIFIC PARTICULATE EMISSION RATE $(\mathrm{g} / \mathrm{hp}-\mathrm{hr})$

.549 


$\begin{array}{ll}\text { LOCOMOTIVE MODEL } & =\text { EMD GP38 } \\ \text { LOCOHOTIVE UNIT \# } & =\text { CSX } \# 2629 \\ \text { THROTTLE NOTCH } & =\text { Notch } 8 \\ \text { TEST DATE } & =10 / 30 / 0 \\ \text { TEST NUMBER } & =10 / 10 \\ \text { SWRI FUEL CODE } & =\text { EM-3004.F } \\ \text { BAROMETER } & =29.25(\mathrm{in} . \mathrm{Hg}) \\ \text { ORY BULB TEMP } & =\mathrm{B} 0 .\left({ }^{\circ} \mathrm{F}\right) \\ \text { ABS HUMIDITY } & =106.84(\mathrm{GR} / \mathrm{LB})\end{array}$

\section{ENGINE PERFORMANCE SUMMARY:}

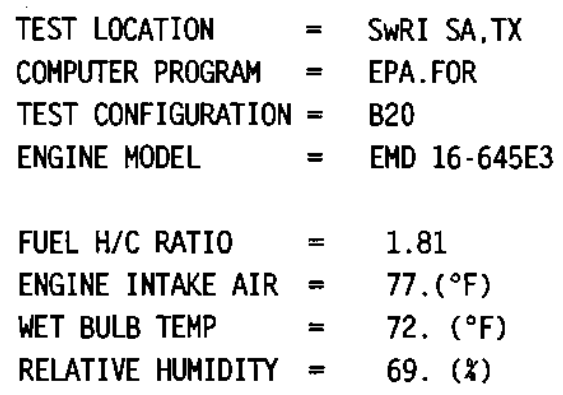

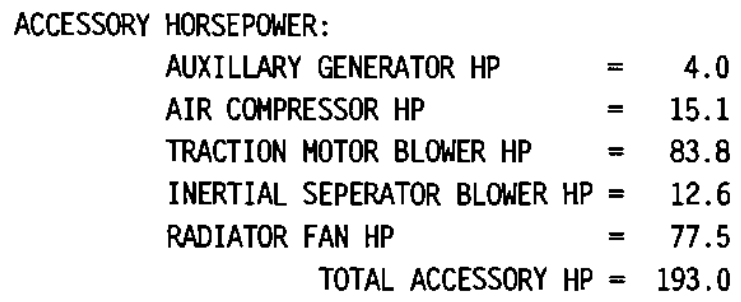




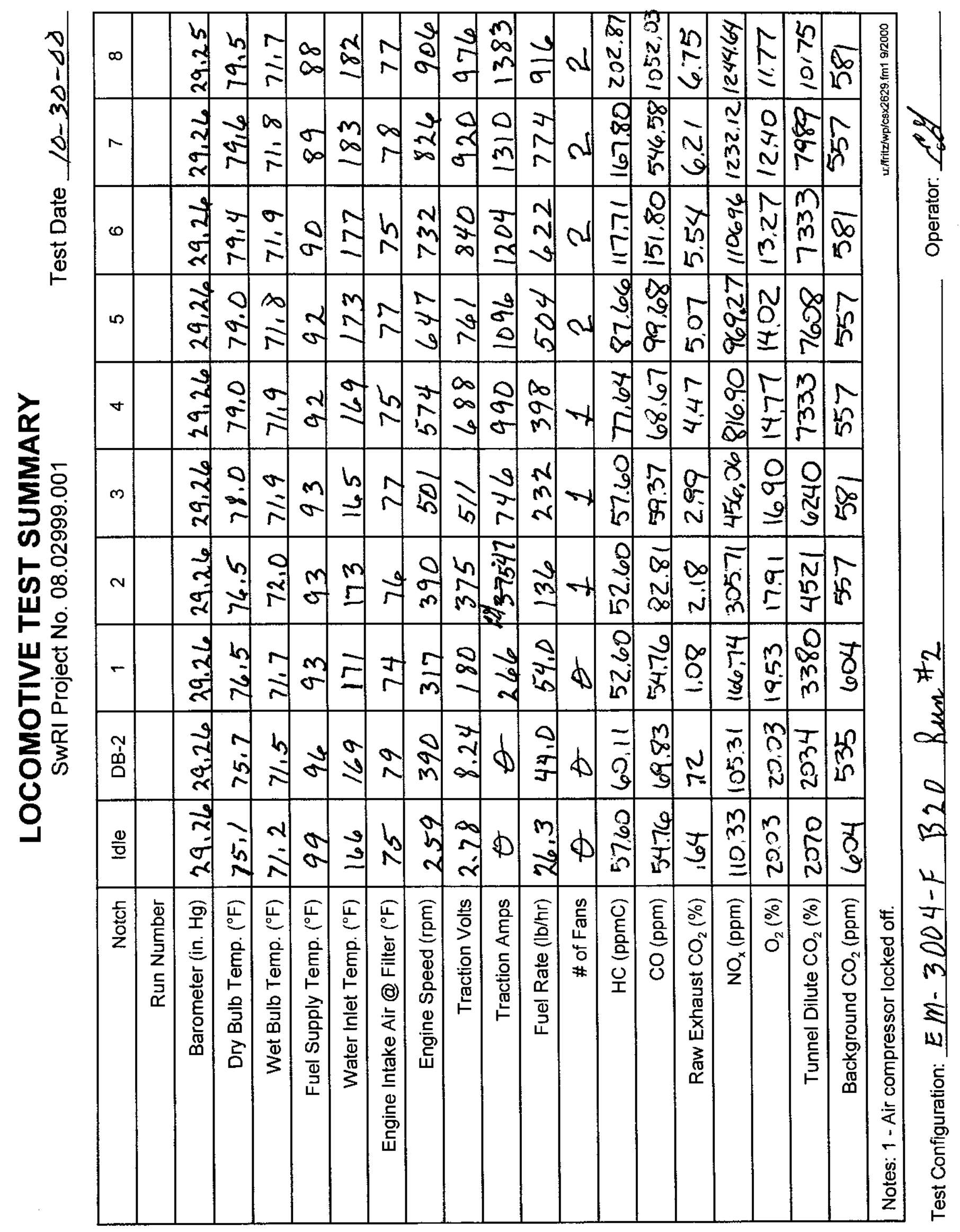




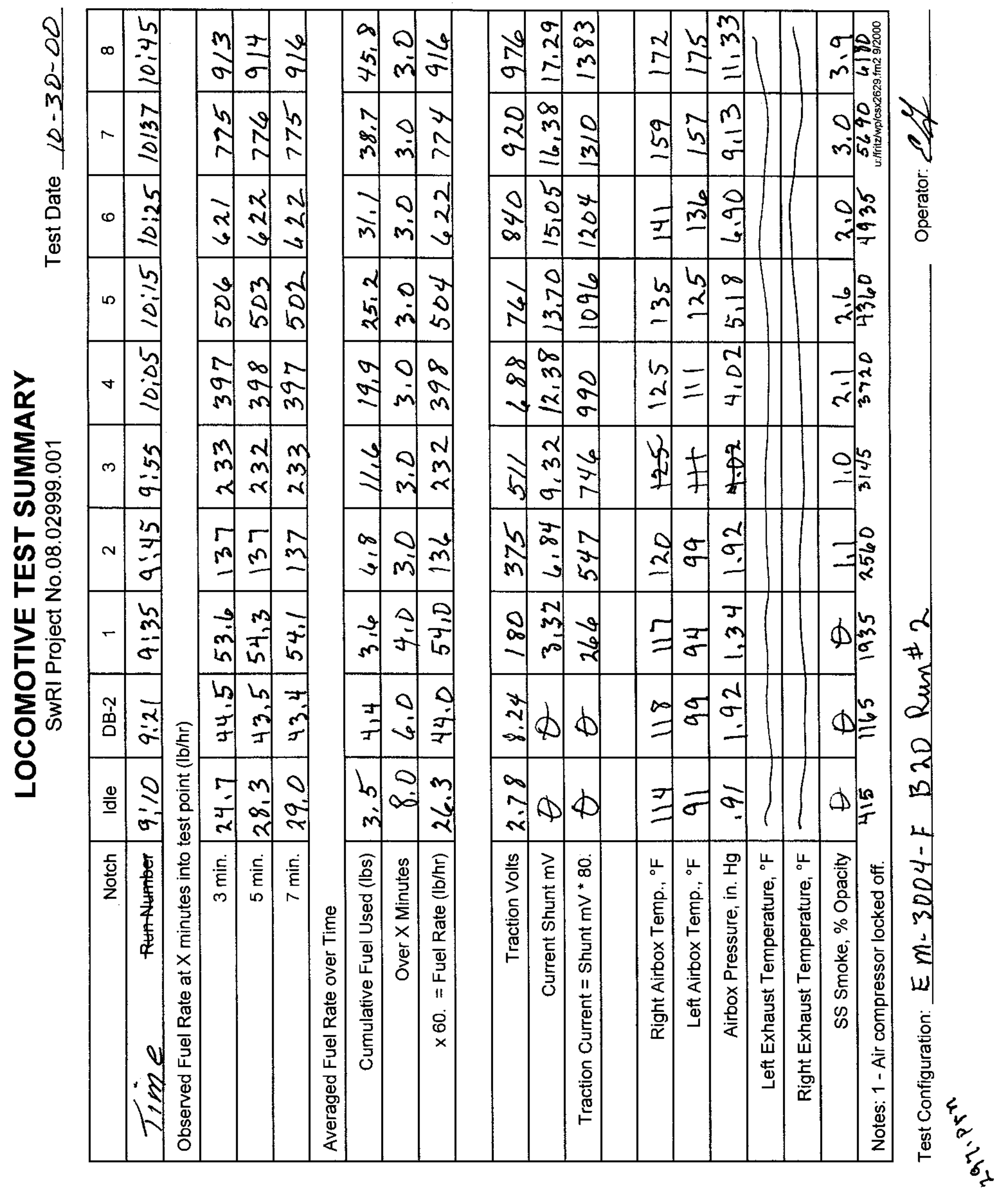




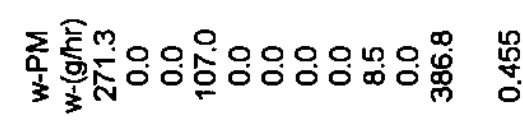

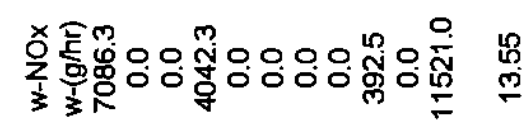

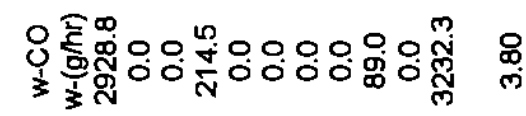

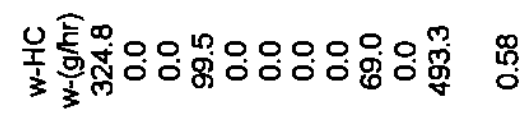

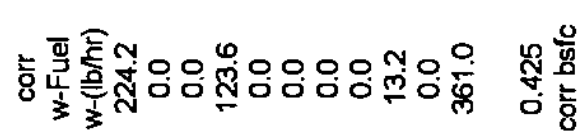

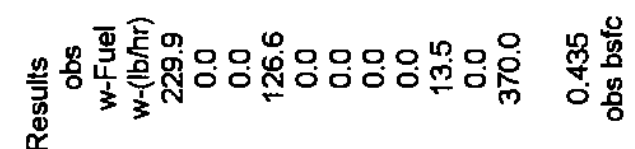

呈

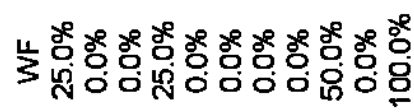

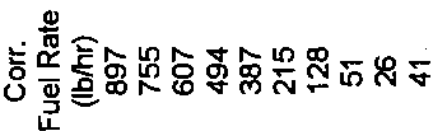

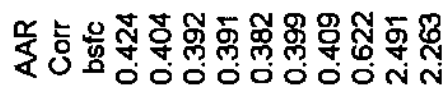

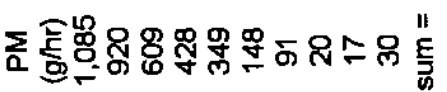

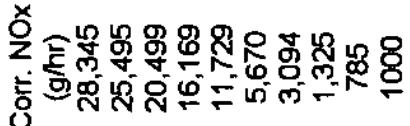

章

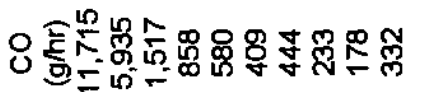

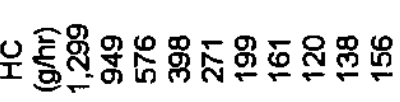

荧

$\sum_{i=1}^{0}$

眾

怘 $\sum_{n=1}$

空产

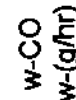

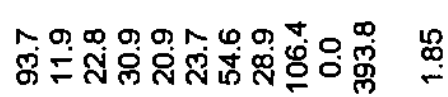

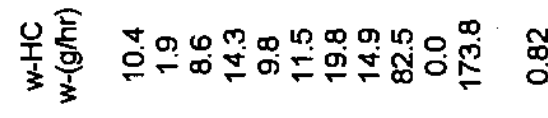

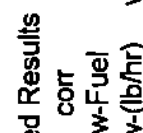

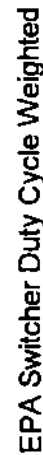

๙

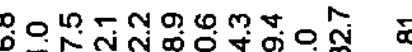

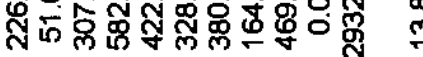

กุำ

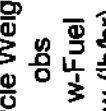

$\frac{0}{1}$
$\frac{1}{1}$
$\frac{1}{3}$

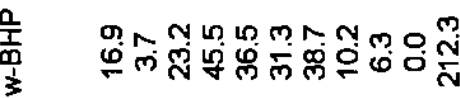

崖

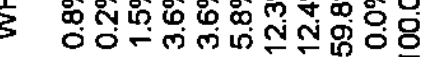

ํํㄴ

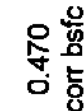

总㩊

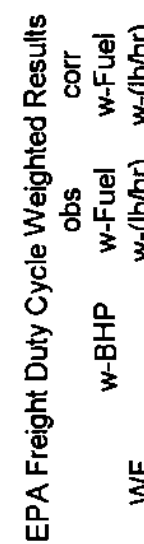

$\frac{0}{3}$

产

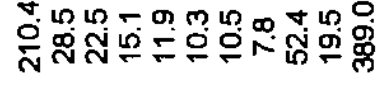

$\stackrel{\circ}{\circ}$

窐

莳

高

○ 흥

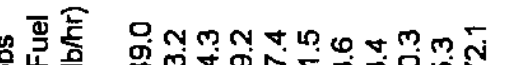

经递

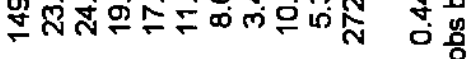

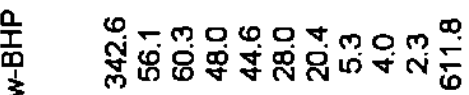

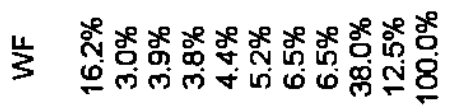




$\begin{array}{ll}\text { LOCOMOTIVE MODEL } & =\text { EMD GP38 } \\ \text { LOCOMOTIVE UNIT \# } & =\text { CSX \#2629 } \\ \text { THROTTLE NOTCH } & =\text { IDLE } \\ \text { TEST DATE } & =11 / 1 / 0 \\ \text { TEST NUMBER } & =1 / 10 \\ \text { SWRI FUEL CODE } & =\text { EM-3004-F } \\ \text { BAROMETER } & =29.13(\mathrm{in} . \mathrm{Hg}) \\ \text { DRY BULB TEMP } & =82 .\left({ }^{\circ} \mathrm{F}\right) \\ \text { ABS HUMIDITY } & =106.87(\mathrm{GR} / \mathrm{LB})\end{array}$

ENGINE PERFORMANCE SUMMARY:

ACCESSORY HORSEPOWER:

$\begin{array}{rlr}\text { AUXILLARY GENERATOR HP } & =4.0 \\ \text { AIR COMPRESSOR HP } & =4.3 \\ \text { TRACTION MOTOR BLOWER HP } & =1.9 \\ \text { INERTIAL SEPERATOR BLOWER HP } & =3 \\ \text { RADIATOR FAN HP } & = \\ \text { TOTAL ACCESSORY HP } & =10.6\end{array}$

$$
\begin{aligned}
& \text { TEST LOCATION = SWRI SA,TX } \\
& \text { COMPUTER PROGRAM = EPA.FOR } \\
& \text { TEST CONFIGURATION }=\text { B2O } \\
& \text { ENGINE HODEL }=\text { EMD 16-645E3 } \\
& \text { FUEL H/C RATIO = } 1.81 \\
& \text { ENGINE INTAKE AIR }=78 .\left(^{\circ} \mathrm{F}\right) \\
& \text { WET BULB TEMP }=72 .\left({ }^{\circ} \mathrm{F}\right) \\
& \text { RELATIVE HUMIDITY }=64 \text {. }(\%)
\end{aligned}
$$

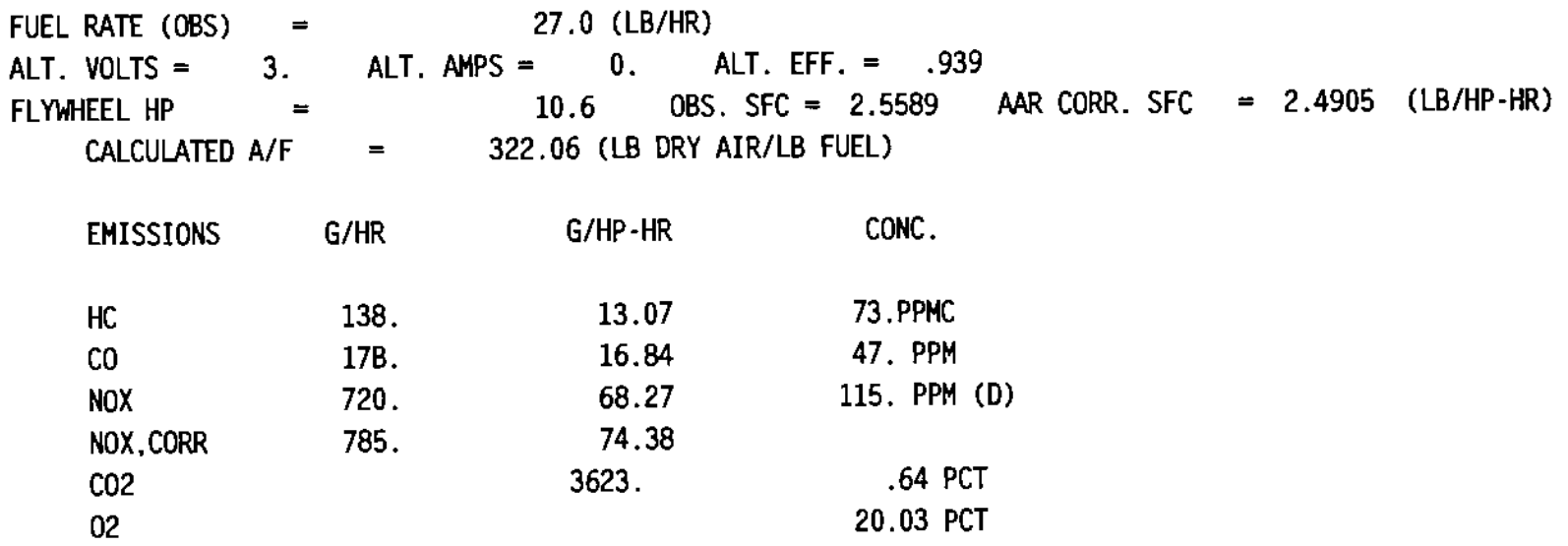

CORRECTION FACTOR SUMMARY:

$\begin{array}{lllll}\text { EPA NOX CF }=1.0896 & \text { NOX-KH } & =1.0732 & \text { NOX-KT } & =1.0000 \\ \text { WET EXH HC CF }=.9891 & \text { AIR TEMP CF }=.9919 & \text { BAROM CF } & =1.0023 \\ \text { FUEL TEMP CF }=.9825 & \text { FUEL S.G. CF }=.9832 & \text { FUEL HHV CF } & =.9789\end{array}$

PARTICULATE INFORMATION

FILTER PAIR I.D. NUMBER

$6812.0 \cdot 97$

FILTER PAIR WEIGHT GAIN (mg)

1.238

SAMPLE VOLUME (scf)

39.216

DILUTE PM CONCENTRATION (mg/scf)

.032

CO2-BASED DILUTION FACTOR

4.685

.148

PARTICULATE CONCENTRATION IN RAW EXHAUST ( $\mathrm{mg} / \mathrm{scf}$ )

PARTICULATE MASS EMISSION RATE $(\mathrm{g} / \mathrm{hr})$

17.

BRAKE-SPECIFIC PARTICULATE EMISSION RATE $(\mathrm{g} / \mathrm{hp}-\mathrm{hr})$

1.627 


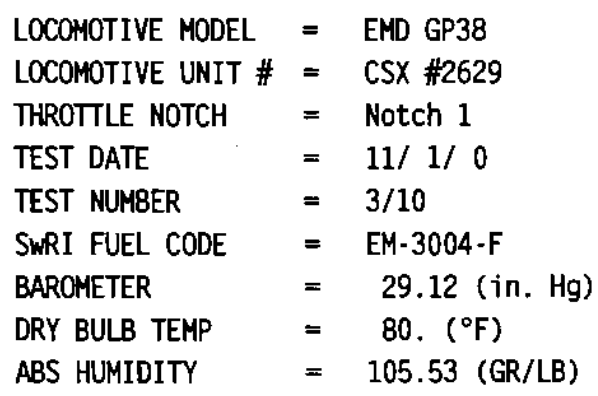

ENGINE PERFORMANCE SUMMARY:

ACCESSORY HORSEPOWER:
AUXILLARY GENERATOR HP $=4.0$
AIR COMPRESSOR HP $=5.3$
TRACTION MOTOR BLOWER HP $=3.6$
INERTIAL SEPERATOR BLOWER HP $=\quad .5$
RADIATOR FAN HP $=.0$
TOTAL ACCESSORY HP $=13.4$

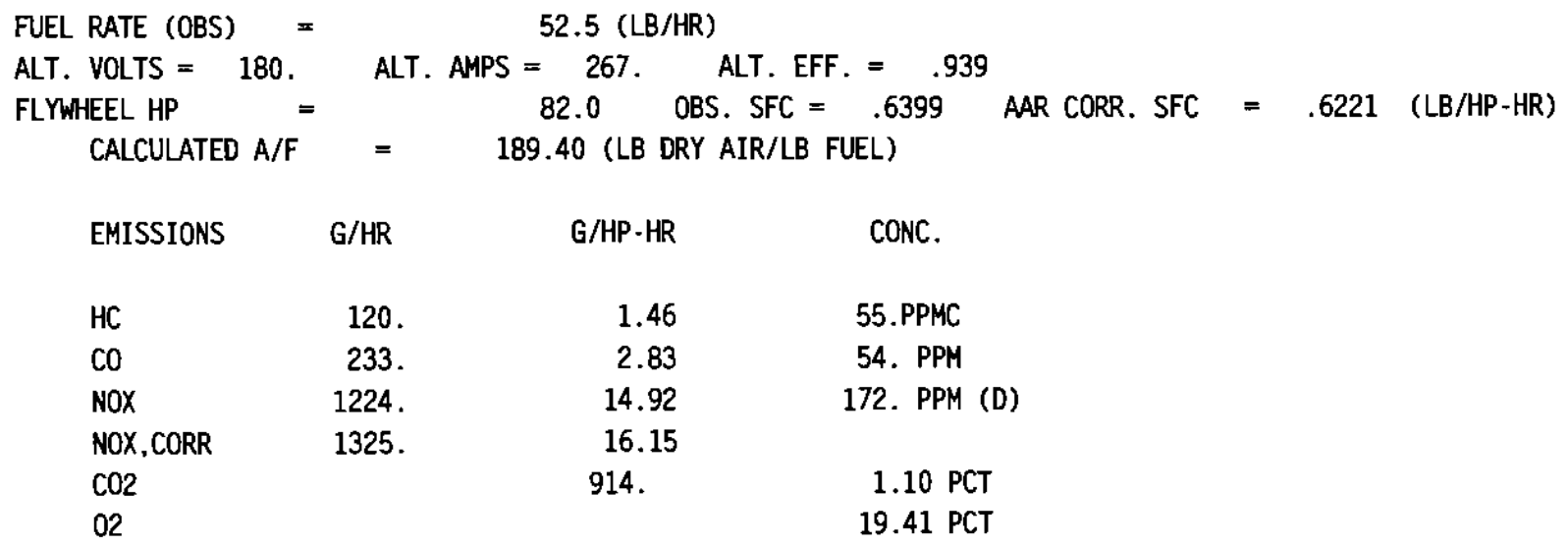

CORRECTION FACTOR SUMMARY:

$\begin{array}{lllll}\text { EPA NOX CF }=1.0825 & \text { NOX-KH } & =1.0674 & \text { NOX-KT } & =1.0000 \\ \text { WET EXH HC CF }=.9851 & \text { AIR TEMP CF }=.9910 & \text { BARON CF } & =1.0023 \\ \text { FUEL TEMP CF }=.9830 & \text { FUEL S.G. CF }=.9832 & \text { FUEL HHV CF }=.9789\end{array}$

PARTICULATE INFORMATION

FILTER PAIR I.D. NUMBER

6814.0 .99

FILTER PAIR WEIGHT GAIN (mg)

1.472

SAMPLE VOLUME (SCf)

38.644

DILUTE PM CONCENTRATION (mg/scf)

.038

CO2-BASED DILUTION FACTOR

3.967

PARTICULATE CONCENTRATION IN RAW EXHAUST (mg/scf)

PARTICULATE MASS EMISSION RATE $(\mathrm{g} / \mathrm{hr})$

.151

BRAKE-SPECIFIC PARTICULATE EMISSION RATE $(\mathrm{g} / \mathrm{hp}-\mathrm{hr})$

20. 


$\begin{array}{llll}\text { LOCOMOTIVE MODEL } & =\text { EMD GP38 } & \text { TEST LOCATION } & =\text { SWRI SA, TX } \\ \text { LOCOMOTIVE UNIT \# } & =\text { CSX \#2629 } & \text { COMPUTER PROGRAM } & =\text { EPA.FOR } \\ \text { THROTTLE NOTCH } & =\text { NOtch } 2 & \text { TEST CONFIGURATION }= & \text { B20 } \\ \text { TEST DATE } & =11 / 1 / 0 & \text { ENGINE MODEL } & =\text { EMD 16-645E3 } \\ \text { TEST NUMBER } & =4 / 10 & & \\ \text { SWRI FUEL CODE } & =\text { EM-3004-F } & \text { FUEL H/C RATIO } & =1.81 \\ \text { BAROMETER } & =29.11(\mathrm{in} . \mathrm{Hg}) & \text { ENGINE INTAKE AIR }=78 .\left({ }^{\circ} \mathrm{F}\right) \\ \text { DRY BULB TEMP } & =81 .\left({ }^{\circ} \mathrm{F}\right) & \text { WET BULB TEMP } & =72 .\left({ }^{\circ} \mathrm{F}\right) \\ \text { ABS HUMIDITY } & =106.88(\mathrm{GR} / \mathrm{LB}) & \text { RELATIVE HUMIDITY } & =65 .(\%)\end{array}$

ENGINE PERFORMANCE SUMMARY:

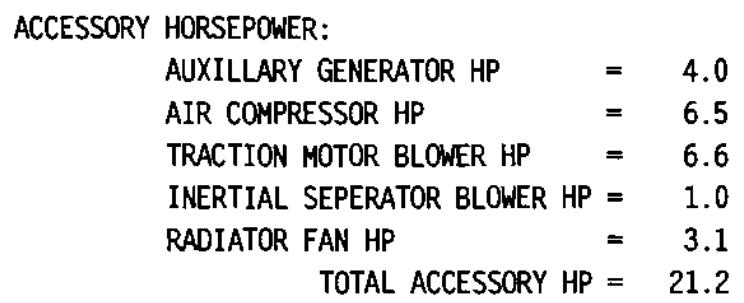




$\begin{array}{llll}\text { LOCOMOTIVE MODEL } & =\text { EMD GP38 } & \text { TEST LOCATION } & =\text { SWRI SA,TX } \\ \text { LOCOMOTIVE UNIT \# } & =\text { CSX \#2629 } & \text { COMPUTER PROGRAM } & =\text { EPA.FOR } \\ \text { THROTTLE NOTCH } & =\text { Notch } 3 & \text { TEST CONFIGURATION } & \text { B20 } \\ \text { TEST DATE } & =11 / 1 / 0 & \text { ENGINE MODEL } & =\text { EMD 16-645E3 } \\ \text { TEST NUMBER } & =5 / 10 & & \\ \text { SWRI FUEL CODE } & =\text { EM-3004-F } & \text { FUEL H/C RATIO } & =1.81 \\ \text { BAROMETER } & =29.10(\mathrm{in} . \mathrm{Hg}) & \text { ENGINE INTAKE AIR } & =77 .\left(^{\circ} \mathrm{F}\right) \\ \text { DRY BULB TEMP } & =81 .\left({ }^{\circ} \mathrm{F}\right) & \text { WET BULB TEMP } & =72 .\left({ }^{\circ} \mathrm{F}\right) \\ \text { ABS HUMIDITY } & =108.80(\mathrm{GR} / \mathrm{LB}) & \text { RELATIVE HUMIDITY } & =67 .(\%)\end{array}$

ENGINE PERFORMANCE SUMMARY:

ACCESSORY HORSEPOWER:

$\begin{array}{llr}\text { AUXILLARY GENERATOR HP } & = & 4.0 \\ \text { AIR COMPRESSOR HP } & =8.3 \\ \text { TRACTION MOTOR BLOWER HP } & =14.0 \\ \text { INERTIAL SEPERATOR BLOWER HP } & =2.1 \\ \text { RADIATOR FAN HP } & 6.5 \\ \text { TOTAL ACCESSORY HP } & =34.9\end{array}$

FUEL RATE (OBS) $=221.0$ (LB/HR)

ALT. VOLTS $=495 . \quad$ ALT. AMPS $=713 . \quad$ ALT. EFF. $=.939$

FLYWHEEL HP $=539.1$ OBS. SFC $=.4100$ AAR CORR. SFC $=.3991$ (LB/HP-HR)

CALCULATED A/F $=70.69$ (LB DRY AIR/LB FUEL)

EMISSIONS G/HR G/HP-HR CONC.

$\begin{array}{lrrr}\text { HC } & 199 . & .37 & 58 . \text { PPMC } \\ \text { CO } & 409 . & .76 & 61 . \text { PPM } \\ \text { NOX } & 5230 . & 9.70 & 471 . \text { PPM (D) } \\ \text { NOX,CORR } & 5670 . & 10.52 & \\ \text { CO2 } & & 589 . & 2.99 \text { PCT } \\ 02 & & & 16.90 \text { PCT }\end{array}$

CORRECTION FACTOR SUMMARY:

$\begin{array}{lllll}\text { EPA NOX CF }=1.0841 & \text { NOX-KH } & =1.0687 & \text { NOX-KT } & =1.0000 \\ \text { WET EXH HC CF }=.9686 & \text { AIR TEMP CF }=.9923 & \text { BAROM CF } & =1.0021 \\ \text { FUEL TEMP CF }=.9835 & \text { FUEL S.G. CF }=.9832 & \text { FUEL HHV CF } & =.9789\end{array}$

PARTICULATE INFORMATION

FILTER PAIR I.D. NUMBER

$6816.0 \cdot 101$

FILTER PAIR WEIGHT GAIN (mg)

4.540

38.241

SAMPLE VOLUME (scf)

.119

5.903

CO2-BASED DILUTION FACTOR

.701

PARTICULATE CONCENTRATION IN RAW EXHAUST ( $\mathrm{mg} / \mathrm{scf}$ )

148.

PARTICULATE MASS EMISSION RATE $(\mathrm{g} / \mathrm{hr})$

.274 


$\begin{array}{llll}\text { LOCOMOTIVE MODEL } & =\text { EMD GP38 } & \text { TEST LOCATION } & =\text { SWRI SA, TX } \\ \text { LOCOMOTIVE UNIT \# } & =\text { CSX \#2629 } & \text { COMPUTER PROGRAM } & =\text { EPA.FOR } \\ \text { THROTTLE NOTCH } & =\text { NOtch } 4 & \text { TEST CONFIGURATION } & \text { B20 } \\ \text { TEST DATE } & =11 / 1 / 0 & \text { ENGINE MODEL } & = \\ \text { TEST NUMBER } & =6 / 10 & & \\ \text { SWRI FUEL CODE } & =\text { EM-3004-F } & \text { FUEL H/C RATIO } & =16-645 E 3 \\ \text { BAROMETER } & =29.11(\mathrm{in} . \mathrm{Hg}) & \text { ENGINE INTAKE AIR } & =70 .\left({ }^{\circ} \mathrm{F}\right) \\ \text { DRY BULB TEMP } & =78 .\left({ }^{\circ} \mathrm{F}\right) & \text { WET BULB TEMP } & =73 .\left({ }^{\circ} \mathrm{F}\right) \\ \text { ABS HUMIDITY } & =115.56(\mathrm{GR} / \mathrm{LB}) & \text { RELATIVE HUMIDITY }=78 .\left({ }^{\circ}\right)\end{array}$

ENGINE PERFORMANCE SUMMARY:

ACCESSORY HORSEPOWER:

$\begin{array}{llr}\text { AUXILLARY GENERATOR HP } & =4.0 \\ \text { AIR COMPRESSOR HP } & =9.6 \\ \text { TRACTION MOTOR BLOWER HP } & =21.2 \\ \text { INERTIAL SEPERATOR BLOWER HP } & =3.2 \\ \text { RADIATOR FAN HP } & 9.8 \\ \text { TOTAL ACCESSORY HP } & =47.7\end{array}$

FUEL RATE (OBS) = 396.0 (LB/HR)

ALT. VOLTS $=683 . \quad$ ALT. AMPS $=990 . \quad$ ALT. EFF. $=.939$

FLYWHELL HP $=1013.6 \quad$ OBS. SFC $=.3907 \quad$ AAR CORR. SFC $=.3815$ (LB/HP-HR)

CALCULATED A/F $=47.22$ (LB DRY AIR/LB FUEL)

EMISSIONS G/HR G/HP-HR CONC.

$\begin{array}{lrrr}\text { HC } & 271 . & .27 & 65 . \text { PPMC } \\ \text { CO } & 580 . & .57 & 72 . \text { PPM } \\ \text { NOX } & 10651 . & 10.51 & 807 . \text { PPM (D) } \\ \text { NOX,CORR } & 11729 . & 11.57 & \\ \text { CO2 } & & 562 . & 4.51 \text { PCT } \\ 02 & & & 14.77 \text { PCT }\end{array}$

CORRECTION FACTOR SUMMARY:

$\begin{array}{lllll}\text { EPA NOX CF }=1.1012 & \text { NOX-KH } & =1.0825 & \text { NOX-KT } & =1.0000 \\ \text { WET EXH HC CF }=.9555 & \text { AIR TEMP CF }=.9955 & \text { BAROM CF } & =1.0022 \\ \text { FUEL TEMP CF }=.9850 & \text { FUEL S.G. CF }=.9832 & \text { FUEL HHV CF } & =.9789\end{array}$

PARTICULATE INFORMATION

FILTER PAIR I.D. NUMBER

$6817.0-102$

FILTER PAIR WEIGHT GAIN (mg)

9.985

37.662

DILUTE PM CONCENTRATION (mg/scf) .265

CO2-BASED DILUTION FACTOR

5.179

1.373

PARTICULATE CONCENTRATION IN RAW EXHAUST (mg/scf) 349.

BRAKE-SPECIFIC PARTICULATE EMISSION RATE $(\mathrm{g} / \mathrm{hp}-\mathrm{hr})$ 


$\begin{array}{ll}\text { LOCOMOTIVE MODEL } & =\text { EMD GP38 } \\ \text { LOCOMOTIVE UNIT \# } & =\text { CSX } \# 2629 \\ \text { THROTTLE NOTCH } & =\text { Notch } 5 \\ \text { TEST DATE } & =11 / 1 / 0 \\ \text { TEST NUMBER } & =7 / 10 \\ \text { SWRI FUEL CODE } & =\text { EM-3004-F } \\ \text { BAROMETER } & =29.11(\text { in. Hg) } \\ \text { DRY 8UL8 TEMP } & =76 .\left({ }^{\circ} \mathrm{F}\right) \\ \text { ABS HUMIDITY } & =112.79(\mathrm{GR} / \mathrm{L} 8)\end{array}$

ENGINE PERFORMANCE SUMMARY:

ACCESSORY HDRSEPOWER:

$\begin{array}{rrr}\text { AUXILLARY GENERATOR HP } & =4.0 \\ \text { AIR COMPRESSOR HP } & =10.8 \\ \text { TRACTION MOTOR BLOWER HP } & =30.5 \\ \text { INERTIAL SEPERATOR 8LOWER HP } & =4.6 \\ \text { RADIATOR FAN HP } & 28.2 \\ \text { TOTAL ACCESSORY HP } & =78.0\end{array}$

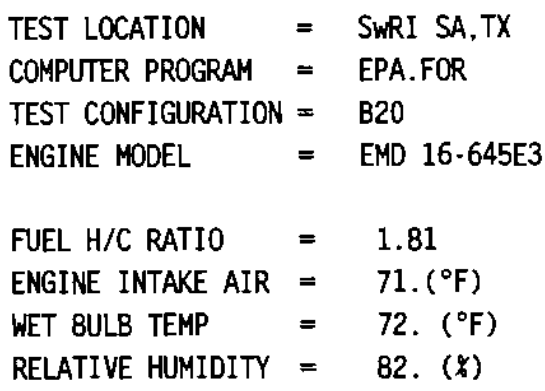

FUEL RATE (OBS) =

ALT. VOLTS $=759$.

FLYWHEEL HP =

CALCULATED A/F

EMISSIONS

G/HR

ALT. AMPS $=1094$.

1264.2

ALT, EFF. $=.939$

42.75 (LB DRY AIR/LB FUEL)
OBS. $\mathrm{SFC}=.4006$

AAR CORR. SFC $=.3910$ (LB/HP-HR)

$\begin{array}{lrrr}\text { HC } & 398 . & .32 & \text { 83.PPMC } \\ \text { CO } & 858 . & .68 & 92 . \text { PPM } \\ \text { NOX } & 14781 . & 11.69 & 969 . \text { PPM (D) } \\ \text { NOX,CORR } & 16169 . & 12.79 & \\ \text { C02 } & & 576 . & 4.99 \text { PCT } \\ 02 & & 14.02 \text { PCT }\end{array}$

CORRECTION FACTOR SUMMARY:

EPA NOX CF $=1.0939$

WET EXH HC CF $=.9517$

FUEL TEMP CF $=.9845$ $\begin{array}{ll}\text { NOX-KT } & =1.0000 \\ \text { BAROM CF } & =1.0022 \\ \text { FUEL HHV CF } & =.9789\end{array}$

PARTICULATE INFORMATION

FILTER PAIR I.D. NUMBER

$6818.0 \cdot 103$

9.060

37.368

.242

5.990

1.452

428.

.339 


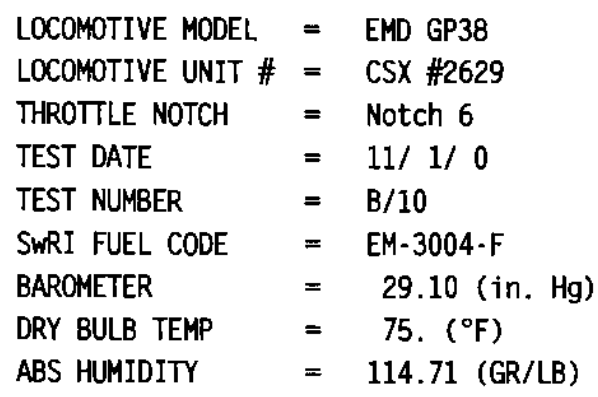

ENGINE PERFORMANCE SUMMARY:

ACCESSORY HORSEPOWER:

$\begin{array}{rlr}\text { AUXILLARY GENERATOR HP } & = & \mathbf{4 . 0} \\ \text { AIR COMPRESSOR HP } & = & 12.2 \\ \text { TRACTION MOTOR BLOWER HP } & = & 44.0 \\ \text { INERTIAL SEPERATOR BLOWER HP } & = \\ \text { RADIATOR FAN HP } & =40.6 \\ \text { TOTAL ACCESSORY HP } & =107.4\end{array}$

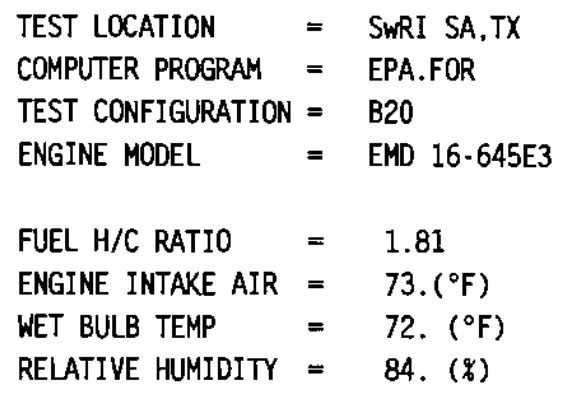

FUEL RATE (OBS) $=622.0(\mathrm{LB} / \mathrm{HR})$

ALT. VOLTS $=838 . \quad$ ALT. AMPS $=1202 . \quad$ ALT. EFF. $=.939$

FLYWHEEL HP $=1546.4$ OBS. SFC $=.4022$ AAR CORR. SFC $=.3923(\mathrm{LB} / \mathrm{HP}-\mathrm{HR})$

CALCULATED A/F $=38.56$ (LB DRY AIR/LB FUEL)

$\begin{array}{lrrr}\text { EMISSIONS } & \text { G/HR } & \text { G/HP.HR } & \text { CONC. } \\ \text { HC } & 576 . & .37 & 10 B . P P M C \\ \text { CO } & 1517 . & .98 & 148 . \text { PPM } \\ \text { NOX } & 18655 . & 12.06 & 1107 . \text { PPM (D) } \\ \text { NOX, CORR } & 20499 . & 13.26 & \\ \text { CO2 } & & 578 . & 5.54 \text { PCT } \\ \text { O2 } & & & 13.27 \text { PCT }\end{array}$

CORRECTION FACTOR SUMMARY:

$\begin{array}{lllll}\text { EPA NOX CF }=1.0988 & \text { NOX-KH } & =1.0806 & \text { NOX-KT } & =1.0000 \\ \text { WET EXH HC CF }=.9471 & \text { AIR TEMP CF }=.9941 & \text { BAROM CF } & =1.0021 \\ \text { FUEL TEMP CF }=.9850 & \text { FUEL S.G. CF }=.9832 & \text { FUEL HHV CF } & =.9789\end{array}$

PARTICULATE INFORMATION

FILTER PAIR I.D. NUMBER

FILTER PAIR WEIGHT GAIN (mg)

6819.0-104

SAMPLE VOLUME (sCf)

DILUTE PM CONCENTRATION (mg/sCf)

9.911

36.933

.268

CO2-BASED DILUTION FACTOR

6.928

PARTICULATE CONCENTRATION IN RAW EXHAUST (mg/scf)

PARTICULATE MASS EMISSION RATE $(\mathrm{g} / \mathrm{hr})$

1.859

BRAKE-SPECIFIC PARTICULATE EMISSION RATE $(\mathrm{g} / \mathrm{hp}-\mathrm{hr})$

609. 


$\begin{array}{ll}\text { LOCOMOTIVE MODEL } & =\text { EMD GP38 } \\ \text { LOCOMOTIVE UNIT \# } & \text { CSX } \# 2629 \\ \text { THROTTLE NOTCH } & =\text { Notch } 7 \\ \text { TEST DATE } & =11 / 1 / 0 \\ \text { TEST NUMBER } & =9 / 10 \\ \text { SWRI FUEL CODE } & =\text { EM-3004-F } \\ \text { BAROMETER } & =29.10(\mathrm{in} . \mathrm{Hg}) \\ \text { DRY BULB TEMP } & =75 .\left({ }^{\circ} \mathrm{F}\right) \\ \text { ABS HUMIDITY } & =114.71 \text { (GR/LB) }\end{array}$

ENGINE PERFORMANCE SUMMARY:

ACCESSORY HORSEPOWER:

$\begin{array}{rrr}\text { AUXILLARY GENERATOR HP } & = & 4.0 \\ \text { AIR COMPRESSOR HP } & =13.8 \\ \text { TRACTION MOTOR BLOWER HP } & =63.7 \\ \text { INERTIAL SEPERATOR BLOWER HP } & =9.6 \\ \text { RADIATOR FAN HP } & 58.9 \\ \text { TOTAL ACCESSORY HP } & =149.9\end{array}$

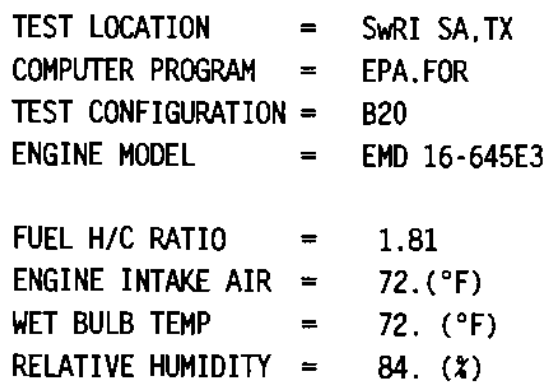

FUEL RATE (OBS) = $774.0(\mathrm{LB} / \mathrm{HR})$

ALT. VOLTS $=919 . \quad$ ALT. AMPS $=1310 . \quad$ ALT. EFF. $=.939$

FLYWEEL HP $=1869.8$ OBS. SFC $=.4140 \quad$ AAR CORR. SFC $=.4039$ (LB/HP-HR)

CALCULATED A/F $=34.71$ (LB DRY AIR/LB FUEL)

EMISSIONS G/HR G/HP-HR CONC.

$\begin{array}{llll}\text { HC } & 949 . & .51 & 158 . P P M C\end{array}$

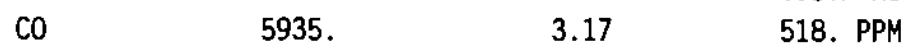

NOX 23202. 12.41 1232. PPM (D)

NOX, CORR 25495 . 13.64

$\begin{array}{ll}\mathrm{CO} 2 & 591 .\end{array}$

$02 \quad 12.52$ PCT

CORRECTION FACTOR SUMMARY:

$\begin{array}{lllll}\text { EPA NOX CF }=1.0988 & \text { NOX-KH } & =1.0806 & \text { NOX-KT } & =1.0000 \\ \text { WET EXH HC CF }=.9421 & \text { AIR TEMP CF }=.9946 & \text { BAROM CF } & =1.0021 \\ \text { FUEL TEMP CF }=.9860 & \text { FUEL S.G. CF }=.9832 & \text { FUEL HHV CF } & =.9789\end{array}$

PARTICULATE INFORMATION

FILTER PAIR I.D. NUMBER

$6820.0-105$

FILTER PAIR WEIGHT GAIN (mg)

12.858

SAMPLE VOLUME (SCf)

36.384

DILUTE PM CONCENTRATION (mg/scf)

.353

CO2-BASED DILUTION FACTOR

7.079

PARTICULATE CONCENTRATION IN RAW EXHAUST (mg/scf)

2.502

PARTICULATE MASS EMISSION RATE $(\mathrm{g} / \mathrm{hr})$

920 .

BRAKE-SPECIFIC PARTICULATE EMISSION RATE $(g / h p-h r)$ 


$\begin{array}{lll}\text { LOCOMOTIVE MODEL } & =\text { EMD GP38 } \\ \text { LOCOMOTIVE UNIT \# } & =\text { CSX } \# 2629 \\ \text { THROTTLE NOTCH } & =\text { Notch } 8 \\ \text { TEST DATE } & =11 / 1 / 0 \\ \text { TEST NUMBER } & =10 / 10 \\ \text { SWRI FUEL CODE } & =\mathrm{EH} \cdot 3004-\mathrm{F} \\ \text { BAROMETER } & =29.10(\mathrm{in} . \mathrm{Hg}) \\ \text { DRY BULB TEMP } & =76 .\left({ }^{\circ} \mathrm{F}\right) \\ \text { ABS HUMIDITY } & =115.80(\mathrm{GR} / \mathrm{LB})\end{array}$

\section{ENGINE PERFORMANCE SUMMARY:}

$\begin{array}{ll}\text { TEST LOCATION } & =\text { SWRI SA,TX } \\ \text { COMPUTER PROGRAM } & =\text { EPA. FOR } \\ \text { TEST CONFIGURATION } & =\text { B20 } \\ \text { ENGINE MODEL } & =\text { EMD } 16.645 E 3 \\ \text { FUEL H/C RATIO } & =1.81 \\ \text { ENGINE INTAKE AIR } & =73 .\left(^{\circ} \mathrm{F}\right) \\ \text { WET BULB TEMP } & =72 .\left({ }^{\circ} \mathrm{F}\right) \\ \text { RELATIVE HUMIDITY } & =84 .(\%)\end{array}$

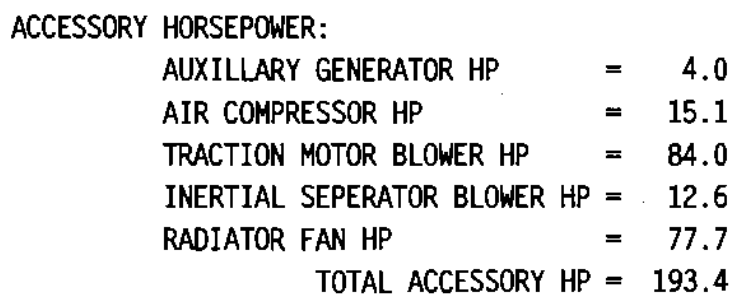




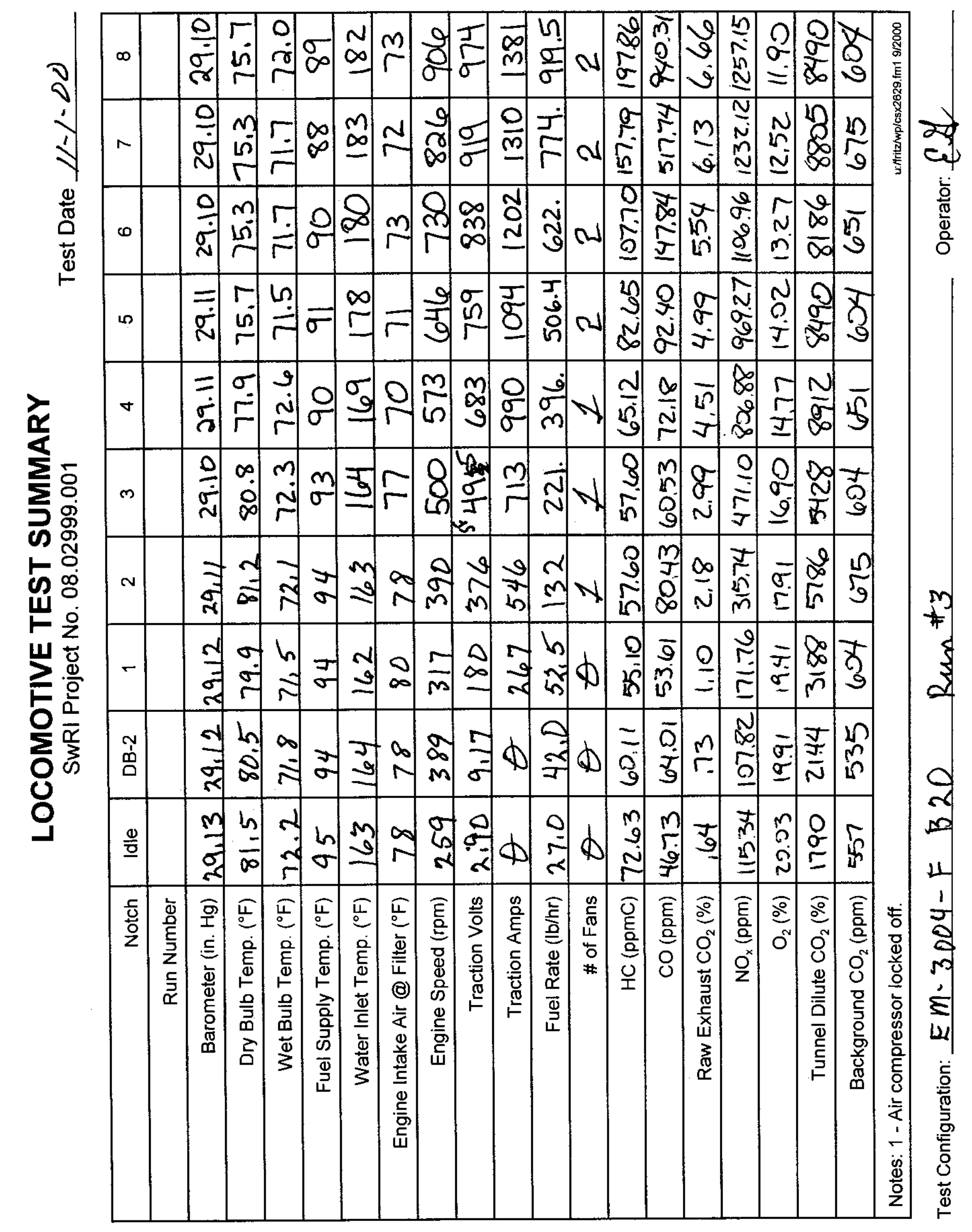




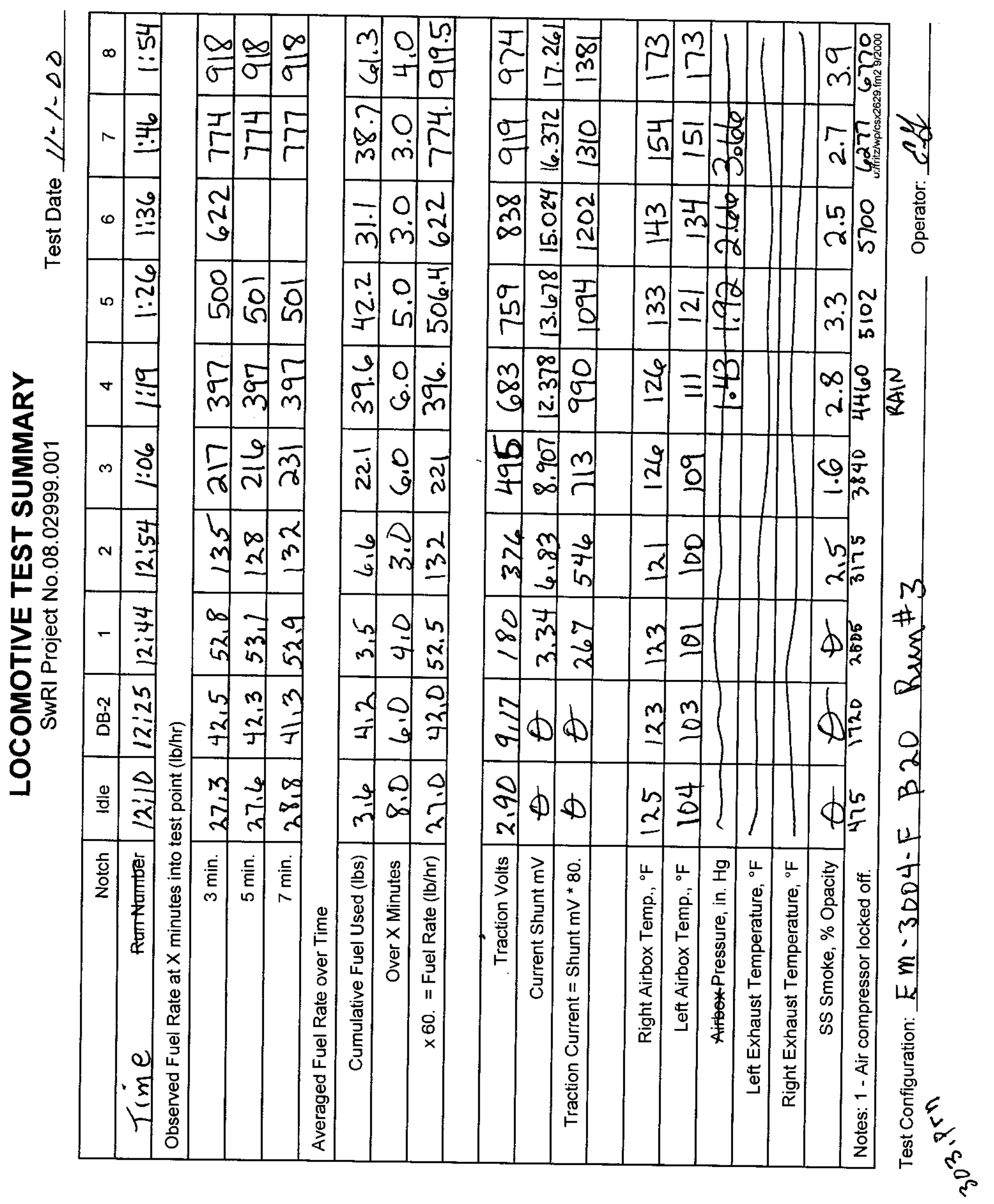




\section{APPENDIX D}

C20 TEST RESULTS 


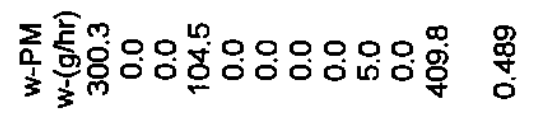

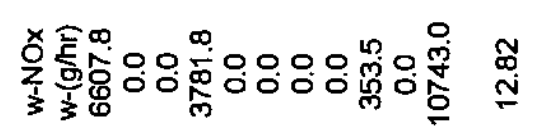

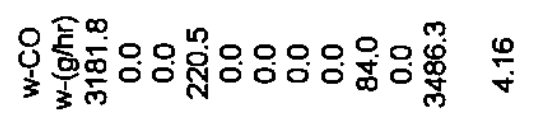

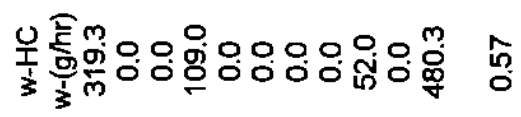

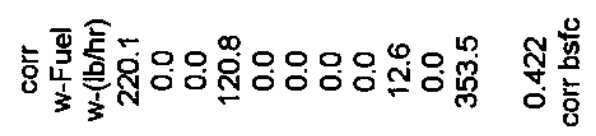

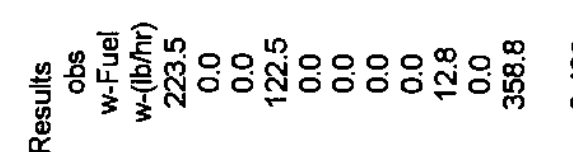

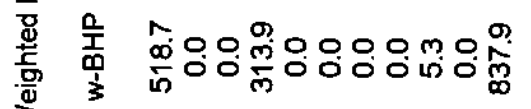

3

m

\&

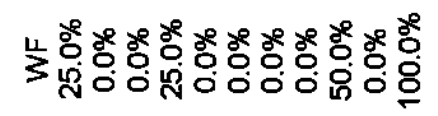

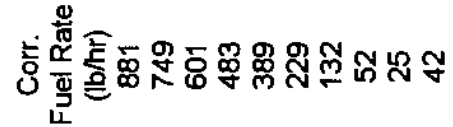

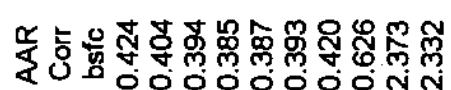

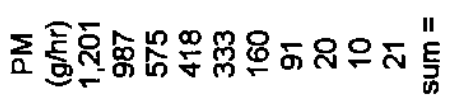

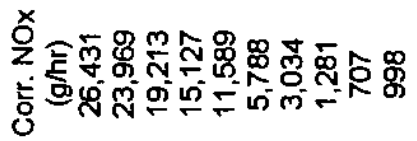

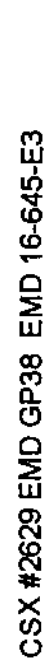

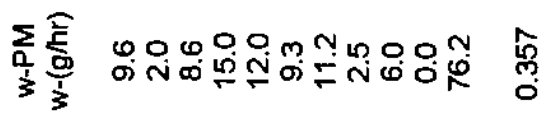

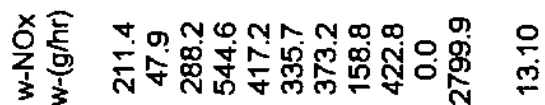

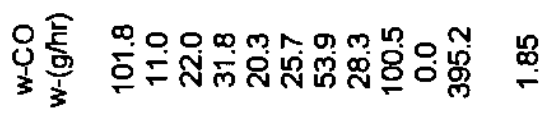

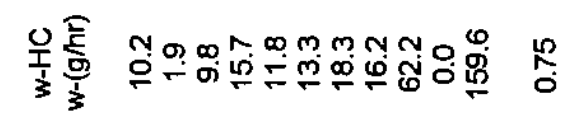

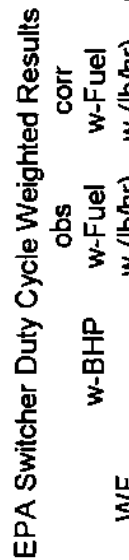

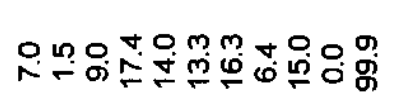

量员

萬

。

.

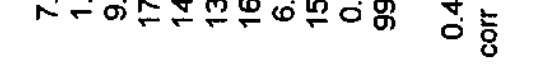

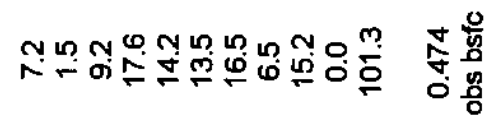

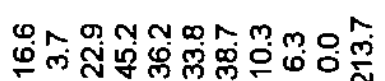

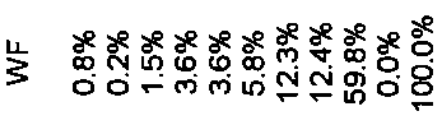

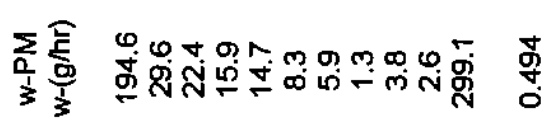

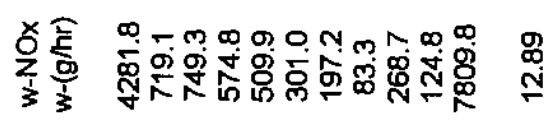

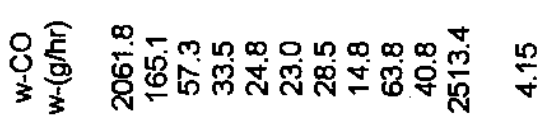

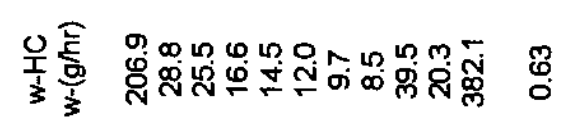

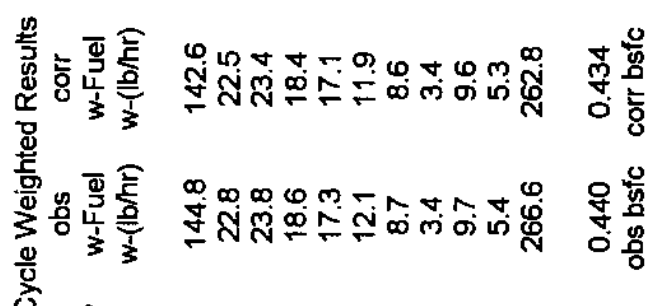

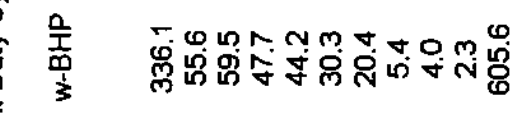

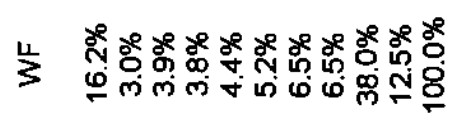

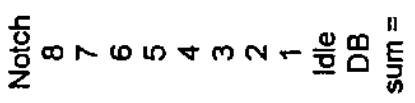

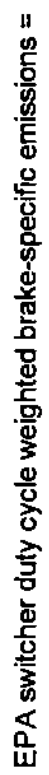

哭 
LOCOMOTIVE EMISSIONS SWRI PROJECT NO. OB-2999-001

$\begin{array}{ll}\text { LOCOMOTIVE MODEL } & =\text { EMD GP3B } \\ \text { LOCOMOTIVE UNIT \# } & =\text { CSX \#2629 } \\ \text { THROTTLE NOTCH } & =\text { IDLE } \\ \text { TEST DATE } & =10 / 27 / 0 \\ \text { TEST NUMBER } & =1 / 10 \\ \text { SWRI FUEL CODE } & =\text { EM-3005-F } \\ \text { BAROMETER } & =29.25(\mathrm{in} . \mathrm{Hg}) \\ \text { DRY BULB TEMP } & =75 .\left(^{\circ} \mathrm{F}\right) \\ \text { ABS HUMIDITY } & =113.51(\mathrm{GR} / \mathrm{LB})\end{array}$

ENGINE PERFORMANCE SUMMARY:

ACCESSORY HORSEPOWER:

$\begin{array}{rlr}\text { AUXILLARY GENERATOR HP } & =4.0 \\ \text { AIR COMPRESSOR HP } & =4.3 \\ \text { TRACTION MOTOR BLOWER HP } & =2.0 \\ \text { INERTIAL SEPERATOR BLOWER HP } & = \\ \text { RADIATOR FAN HP } & .3 \\ \text { TOTAL ACCESSORY HP } & =10.6\end{array}$

FUEL RATE (OBS) =

ALT, VOLTS $=$

3.

25.5 (LB/HR)

FLYWHEEL HP

$=$

ALT. AMPS $=$

0 .

ALT. EFF. $=.939$

$\begin{array}{ll}\text { TEST LOCATION } & =\text { SWRI SA,TX } \\ \text { COMPUTER PROGRAM } & =\text { EPA.FOR } \\ \text { TEST CONFIGURATION } & =\text { C20 } \\ \text { ENGINE MODEL } & =\text { EMD } 16 \cdot 645 E 3 \\ \text { FUEL H/C RATIO } & =1.81 \\ \text { ENGINE INTAKE AIR } & =77 .\left(^{\circ} \mathrm{F}\right) \\ \text { WET BULB TEMP } & =71 .\left({ }^{\circ} \mathrm{F}\right) \\ \text { RELATIVE HUMIDITY } & =\mathrm{B} 6 .(\%)\end{array}$

CALCULATED A/F

EMISSIONS

G/HR

OBS. $\mathrm{SFC}=2.40 \mathrm{BO}$ 322.81 (LB DRY AIR/LB FUEL)

$\begin{array}{lrr}\text { HC } & 104 . & 9.78 \\ \text { CO } & 168 . & 15.88 \\ \text { NOX } & 637 . & 60.19 \\ \text { NOX,CORR } & 707 . & 66.77 \\ \text { CO2 } & & 3418 .\end{array}$

02

CORRECTION FACTOR SUMMARY:

$\begin{array}{lllll}\text { EPA NOX CF }=1.1093 & \text { NOX-KH } & =1.0891 & \text { NOX-KT } & =1.0000 \\ \text { WET EXH HC CF }=.9888 & \text { AIR TEMP CF }=.9923 & \text { BAROM CF } & =1.0034 \\ \text { FUEL TEMP CF }=.9825 & \text { FUEL S.G. CF }=1.0049 & \text { FUEL HHV CF } & =.9896\end{array}$

PARTICULATE INFORMATION

FILTER PAIR I.D. NUMBER

$6732.0 \cdot 17$

.940

38.719

.024

3.814

.093

10.

.961 


$\begin{array}{ll}\text { LOCOMOTIVE MODEL } & =\text { EMD GP38 } \\ \text { LOCOMOTIVE UNIT \# } & =\text { CSX } \# 2629 \\ \text { THROTTLE NOTCH } & =\text { DB2 } \\ \text { TEST DATE } & =10 / 27 / 0 \\ \text { TEST NUMBER } & =2 / 10 \\ \text { SWRI FUEL CODE } & =\text { EM-3005-F } \\ \text { BAROMETER } & =29.25(\mathrm{in} . \mathrm{Hg}) \\ \text { DRY BULB TEMP } & =75 .\left(^{\circ} \mathrm{F}\right) \\ \text { ABS HUMIDITY } & =113.51(\mathrm{GR} / \mathrm{LB})\end{array}$

ENGINE PERFORMANCE SUMMARY:

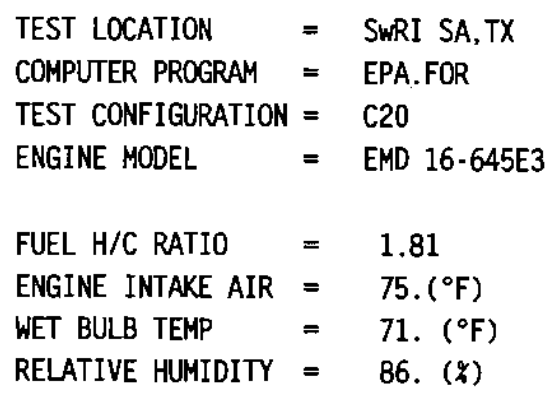

ACCESSORY HORSEPOWER:

$\begin{array}{rlr}\text { AUXILLARY GENERATOR HP } & =4.0 \\ \text { AIR COMPRESSOR HP } & =6.5 \\ \text { TRACTION MOTOR BLOWER HP } & =6.7 \\ \text { INERTIAL SEPERATOR BLOWER HP } & =1.0 \\ \text { RADIATOR FAN HP } & .0 \\ \text { TOTAL ACCESSORY HP } & =18.2\end{array}$

FUEL RATE (OBS) =

ALT. VOLTS $=$

FLYWHEEL HP

8.

$=$

43.0 (LB/HR)

$\begin{array}{lccc}\text { CALCULATED A/F } & = & 18.2 & \text { OBS. SFC }=2.3647 \\ \text { EMISSIONS } & \text { G/HR } & \text { G/HP-HR } & \text { CONC. } \\ \text { HC } & & & \\ \text { CO } & 162 . & 8.91 & 60 . \text { PPHC } \\ \text { NOX } & 326 . & 17.95 & 61 . \text { PPM } \\ \text { NOX,CORR } & 899 . & 49.46 & 102 . \text { PPH (D) } \\ \text { C02 } & 998 . & 54.86 & .72 \text { PCT } \\ \text { O2 } & & 3355 . & 20.03 \text { PCT }\end{array}$

CORRECTION FACTOR SUMMARY:

$\begin{array}{lllll}\text { EPA NOX CF }=1.1090 & \text { NOX-KH } & =1.0889 & \text { NOX-KT } & =1.0000 \\ \text { WET EXH HC CF }=.9881 & \text { AIR TEMP CF }=.9932 & \text { BAROM CF } & =1.0034 \\ \text { FUEL TEMP CF }=.9825 & \text { FUEL S.G. CF }=1.0049 & \text { FUEL HHV CF } & =.9896\end{array}$

PARTICULATE INFORMATION

FILTER PAIR I.D. NUMBER

6733.0 .18
1.219
38.660
.032
4.094
.129
21.
1.170




$\begin{array}{ll}\text { LOCOMOTIVE MODEL } & =\text { EMD GP38 } \\ \text { LOCOMOTIVE UNIT \# } & =\text { CSX } \# 2629 \\ \text { THROTTLE NOTCH } & =\text { Notch } 1 \\ \text { TEST DATE } & =10 / 27 / 0 \\ \text { TEST NUMBER } & =3 / 10 \\ \text { SWRI FUEL CODE } & =\text { EM-3005-F } \\ \text { BAROMETER } & =29.26(\mathrm{in} . \mathrm{Hg}) \\ \text { DRY BULB TEMP } & =76 .\left({ }^{\circ} \mathrm{F}\right) \\ \text { ABS HUMIDITY } & =113.10(\mathrm{GR} / \mathrm{LB})\end{array}$

ENGINE PERFORMANCE SUMMARY:

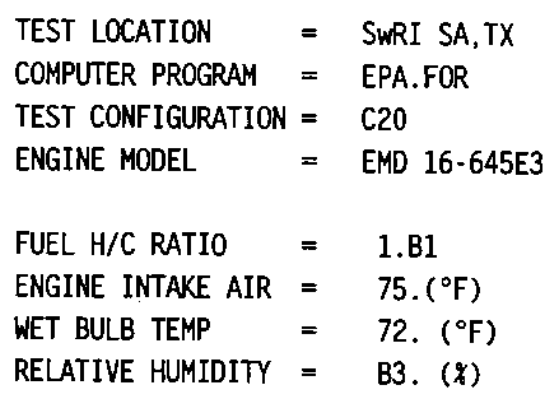

ACCESSORY HORSEPOWER:

$\begin{array}{rlr}\text { AUXILLARY GENERATOR HP } & =4.0 \\ \text { AIR COMPRESSOR HP } & =5.3 \\ \text { TRACTION MOTOR BLOWER HP } & =3.6 \\ \text { INERTIAL SEPERATOR BLOWER HP } & =.5 \\ \text { RADIATOR FAN HP } & =.0 \\ \text { TOTAL ACCESSORY HP } & =13.4\end{array}$

FUEL RATE (08S) = $52.5(\mathrm{LB} / \mathrm{HR})$

ALT. VOLTS $=$ 181. ALT. AMPS $=268 . \quad$ ALT. EFF. $=.939$

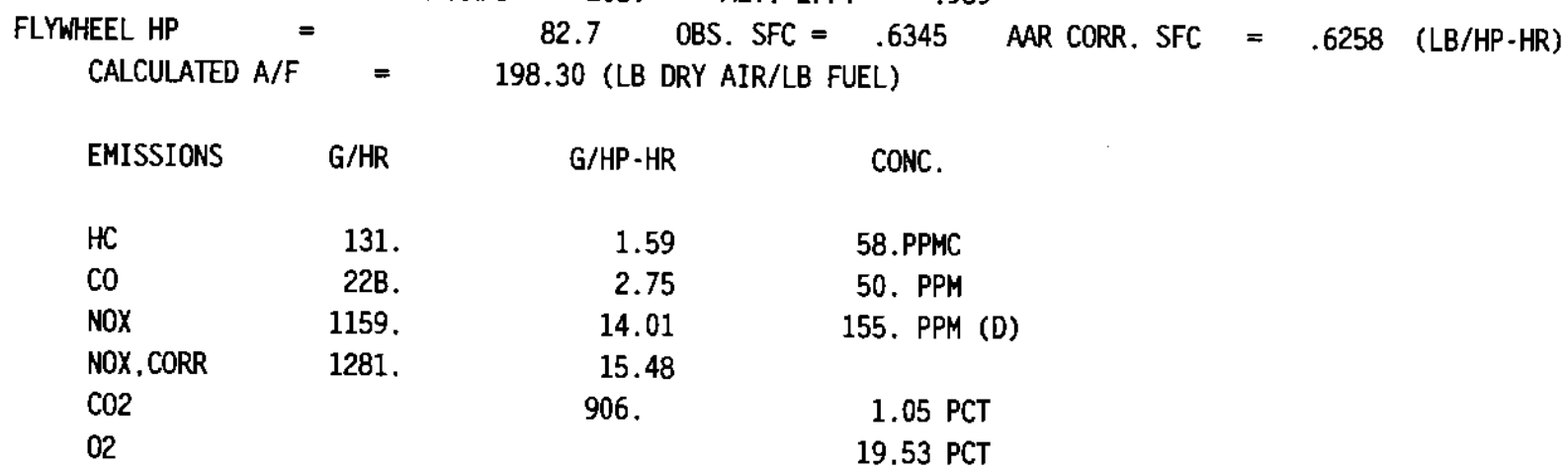

CORRECTION FACTOR SUMMARY:

$\begin{array}{lllll}\text { EPA NOX CF }=1.1046 & \text { NOX-KH } & =1.0853 & \text { NOX-KT } & =1.0000 \\ \text { WET EXH HC CF }=.9852 & \text { AIR TEMP CF }=.9932 & \text { BAROM CF } & =1.0035 \\ \text { FUEL TEMP CF }=.9835 & \text { FUEL S.G. CF }=1.0049 & \text { FUEL HHV CF } & =.9896\end{array}$

PARTICULATE INFORMATION

FILTER PAIR I.D. NUMBER

$6734.0-19$

FILTER PAIR WEIGHT GAIN (mg)

1.665

3В.378

SAMPLE VOLUME (SCF)

.043

3.339

CO2-BASED DILUTION FACTOR

.145

PARTICULATE MASS EMISSION RATE $(g / \mathrm{hr})$

20.

BRAKE-SPECIFIC PARTICULATE EMISSION RATE ( $g / \mathrm{hp}-\mathrm{hr}$ ) 


$\begin{array}{ll}\text { LOCOMOTIVE MODEL } & =\text { EMD GP38 } \\ \text { LOCOMOTIVE UNIT \# } & \text { CSX \#2629 } \\ \text { THROTTLE NOTCH } & =\text { Notch } 2 \\ \text { TEST DATE } & =10 / 27 / 0 \\ \text { TEST NUMBER } & =4 / 10 \\ \text { SWRI FUEL CODE } & =\text { EM-3005-F } \\ \text { BAROMETER } & =29.26(\mathrm{in} . \mathrm{Hg}) \\ \text { ORY BULB TEMP } & =76 .\left({ }^{\circ} \mathrm{F}\right) \\ \text { ABS HUMIDITY } & =112.48(\mathrm{GR} / \mathrm{B})\end{array}$

ENGINE PERFORMANCE SUMMARY:

ACCESSORY HORSEPOWER:

$\begin{array}{rlr}\text { AUXILLARY GENERATOR HP } & =4.0 \\ \text { AIR COMPRESSOR HP } & =6.5 \\ \text { TRACTION MOTOR BLOWER HP } & =6.7 \\ \text { INERTIAL SEPERATOR BLOWER HP } & =1.0 \\ \text { RADIATOR FAN HP } & =3.1 \\ \text { TOTAL ACCESSORY HP } & =21.4\end{array}$

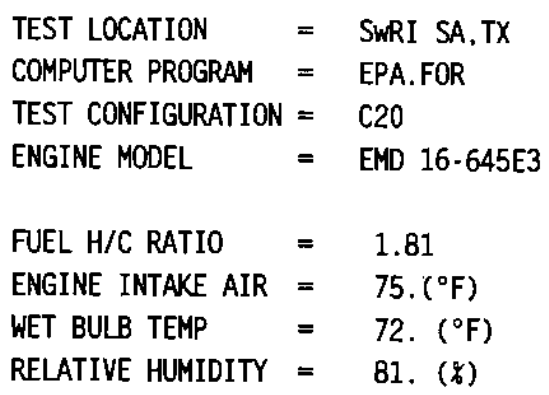

FUEL RATE (OBS) $=134.0$ (LB/HR)

ALT. VOLTS $=376 . \quad$ ALT. AMPS $=$ 546. ALT. EFF. $=.939$

FLYWHEEL HP $=314.6$ OBS. SFC $=.4259$ AAR CORR. SFC $=.4201$ (LB/HP-HR)

CALCULATED A/F $=96.41$ (LB DRY AIR/LB FUEL)

EMISSIONS G/HR G/HP-HR CONC.

HC $\quad 149 . \quad 48 \quad$ 53.PPMC

$\begin{array}{llll}\mathrm{CO} & 438 . & 1.39 & 78 . \mathrm{PPM}\end{array}$

$\begin{array}{llll}\text { NOX } & 2771 . & 8.81 & 301 .\end{array}$

NOX,CORR $\quad 3034 . \quad 9.64$

CO2 611 . 2.18 PCT

$02 \quad 17.91$ PCT

CORRECTION FACTOR SUMMARY:

$\begin{array}{lllll}\text { EPA NOX CF }=1.0947 & \text { NOX-KH } & =1.0773 & \text { NOX-KT } & =1.0000 \\ \text { WET EXH HC CF }=.9754 & \text { AIR TEMP CF }=.9932 & \text { BAROM CF } & =1.0035 \\ \text { FUEL TEMP CF }=.9830 & \text { FUEL S.G. CF }=1.0049 & \text { FUEL HHV CF } & =.9896\end{array}$

PARTICULATE INFORMATION

FILTER PAIR I.D. NUMBER

$6735.0-20$

FILTER PAIR WEIGHT GAIN (mg)

4.701

SAMPLE VOLUME (SCf)

38.300

DILUTE PM CONCENTRATION (mg/scf)

.123

CO2-BASED DILUTION FACTOR

4.269

PARTICULATE CONCENTRATION IN RAW EXHAUST ( $\mathrm{mg} / \mathrm{scf}$ )

.524

PARTICULATE MASS EMISSION RATE $(\mathrm{g} / \mathrm{hr})$

91.

BRAKE-SPECIFIC PARTICULATE EMISSION RATE $(g / h p-h r)$ 


$\begin{array}{ll}\text { LOCOMOTIVE MODEL } & =\text { EMD GP38 } \\ \text { LOCOMOTIVE UNIT \# } & =\text { CSX } \# 2629 \\ \text { THROTTLE NOTCH } & =\text { Notch } 3 \\ \text { TEST DATE } & =10 / 27 / 0 \\ \text { TEST NUMBER } & =5 / 10 \\ \text { SWRI FUEL CODE } & =\text { EM-3005-F } \\ \text { BAROMETER } & =29.27(\mathrm{in} . \mathrm{Hg}) \\ \text { DRY BULB TEMP } & \left.=76 .{ }^{\circ} \mathrm{F}\right) \\ \text { ABS HUMIDITY } & =114.46(\mathrm{GR} / \mathrm{LB})\end{array}$

ENGINE PERFORMANCE SUMMARY:

ACCESSORY HORSEPOWER:

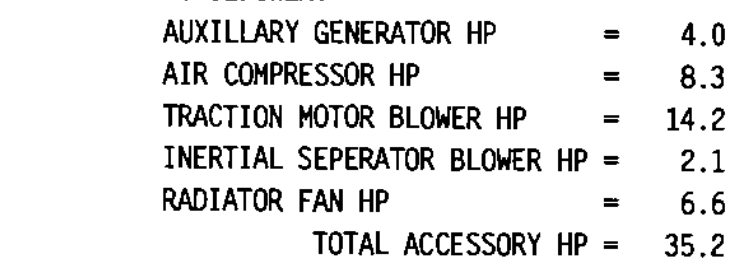

$\begin{array}{rlr}\text { AUXILLARY GENERATOR HP } & = & 4.0 \\ \text { AIR COMPRESSOR HP } & = & 8.3 \\ \text { TRACTION MOTOR BLOWER HP } & =14.2 \\ \text { INERTIAL SEPERATOR BLOWER HP } & =2.1 \\ \text { RADIATOR FAN HP } & = & 6.6 \\ \text { TOTAL ACCESSORY HP } & =35.2\end{array}$

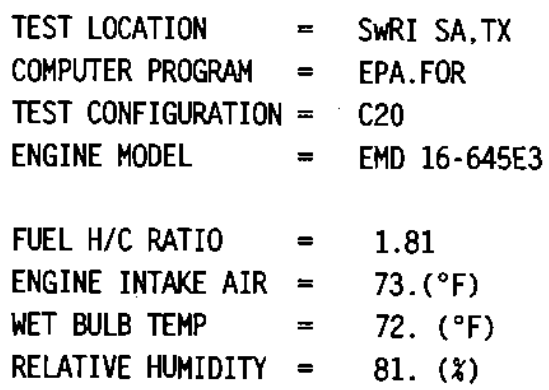

CORRECTION FACTOR SUMMARY:

$\begin{array}{lllll}\text { EPA NOX CF }=1.0989 & \text { NOX-KH } & =1.0806 & \text { NOX-KT } & =1.0000 \\ \text { WET EXH HC CF }=.9687 & \text { AIR TEMP CF }=.9941 & \text { BAROM CF } & =1.0035 \\ \text { FUEL TEMP CF }=.9835 & \text { FUEL S.G. CF }=1.0049 & \text { FUEL HHV CF } & =.9896\end{array}$

PARTICULATE INFORMATION

FILTER PAIR I.D. NUMBER

FILTER PAIR WEIGHT GAIN (mg)

$6736.0-21$

5.614

38.287

DILUTE PM CONCENTRATION (mg/scf)

CO2-BASED DILUTION FACTOR $\quad 4.853$

PARTICULATE CONCENTRATION IN RAW EXHAUST (mg/scf) .712

PARTICULATE MASS EMISSION RATE $(\mathrm{g} / \mathrm{hr})$

160.

BRAKE-SPECIFIC PARTICULATE EMISSION RATE ( $g / h p-h r) \quad .274$ 
LOCONOTIVE EMISSIONS SWRI PROJECT NO. 08-2999-001

$\begin{array}{llll}\text { LOCOMOTIVE MODEL } & =\text { EMD GP38 } & \text { TEST LOCATION } & =\text { SWRI SA,TX } \\ \text { LOCOMOTIVE UNIT \# } & =\text { CSX \#2629 } & \text { COMPUTER PROGRAM } & =\text { EPA.FOR } \\ \text { THROTLE NOTCH } & =\text { Notch } 4 & \text { TEST CONFIGURATION } & \text { C20 } \\ \text { TEST DATE } & =10 / 27 / 0 & \text { ENGINE MODEL } & =\text { EMD } 16-645 E 3 \\ \text { TEST NUMBER } & =6 / 10 & & \\ \text { SWRI FUEL CODE } & =\text { EM-3005-F } & \text { FUEL H/C RATIO } & =1.81 \\ \text { BAROMETER } & =29.27(\mathrm{in} . \mathrm{Hg}) & \text { ENGINE INTAKE AIR } & =74 .\left({ }^{\circ} \mathrm{F}\right) \\ \text { DRY BULB TEMP } & =77 .\left({ }^{\circ} \mathrm{F}\right) & \text { WET BULB TEMP } & =72 .\left({ }^{\circ} \mathrm{F}\right) \\ \text { ABS HUMIDITY } & =111.99(\mathrm{GR} / \mathrm{LB}) & \text { RELATIVE HUMIDITY } & =79 .\left({ }^{\circ}\right)\end{array}$

ENGINE PERFORMANCE SUMMARY:

ACCESSORY HORSEPOWER:

AUXILLARY GENERATOR HP $=4.0$

AIR COMPRESSOR HP $\quad=9.6$

TRACTION MOTOR BLOWER HP $=21.4$

INERTIAL SEPERATOR BLOWER HP $=3.2$

RADIATOR FAN HP $=9.9$

TOTAL ACCESSORY HP $=48.1$

FUEL RATE (OBS) = 394.0 (LB/HR)

ALT. VOLTS $=681 . \quad$ ALT. AMPS $=984 . \quad$ ALT. EFF. $=.939$

FLYHEEL HP $=1005.4 \quad$ OBS. SFC $=.3919 \quad$ AAR CORR. SFC $=.3867$ (LB/HP-HR)

CALCULATED A/F $=48.48$ (LB DRY AIR/LB FUEL)

EMISSIONS G/HR G/HP-HR CONC.

$\begin{array}{lrrr}\text { HC } & 329 . & .33 & 78 . \text { PPMC } \\ \text { CO } & 564 . & .56 & 69 . \text { PPM } \\ \text { NOX } & 10614 . & 10.56 & 787 . \text { PPM (D) } \\ \text { NOX, CORR } & 11589 . & 11.53 & \\ \text { C02 } & & 563 . & 4.39 \text { PCT } \\ 02 & & & 14.90 \text { PCT }\end{array}$

CORRECTION FACTOR SUMMARY:

$\begin{array}{lllll}\text { EPA NOX CF }=1.0919 & \text { NOX-KH } & =1.0750 & \text { NOX-KT } & =1.0000 \\ \text { WET EXH HC CF }=.9567 & \text { AIR TEMP CF }=.9937 & \text { BAROM CF } & =1.0035 \\ \text { FUEL TEMP CF }=.9835 & \text { FUEL S.G. CF }=1.0049 & \text { FUEL HHV CF } & =.9896\end{array}$

PARTICULATE INFORMATION

FILTER PAIR I.D. NUMBER

$6737.0-22$

FILTER PAIR WEIGHT GAIN (mig)

B. 197

37.731

SAMPLE VOLUME (SCf)

.217

5.916

CO2-BASED DILUTION FACTOR

1.285

PARTICULATE CONCENTRATION IN RAW EXHAUST (mg/sCf)

333.

BRAKE-SPECIFIC PARTICULATE EMISSION RATE $(g / h p-h r)$

.332 


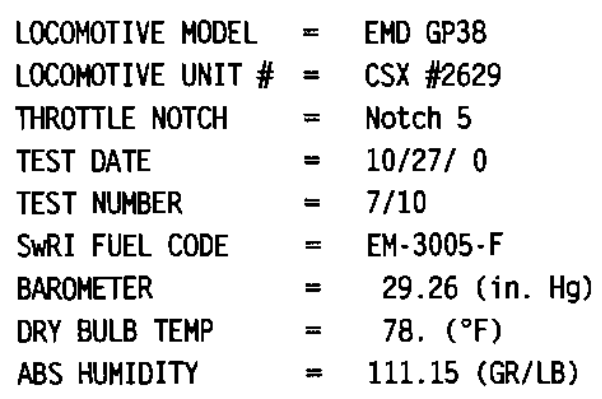

ENGINE PERFORMANCE SUMMARY:

ACCESSORY HORSEPOWER:
AUXILLARY GENERATOR HP $=4.0$
AIR COMPRESSOR HP $\quad=10.8$
TRACTION MOTOR BLOWER HP $=30.6$
INERTIAL SEPERATOR BLOWER HP $=4.6$
RADIATOR FAN HP $=28.3$
TOTAL ACCESSORY HP $=78.3$

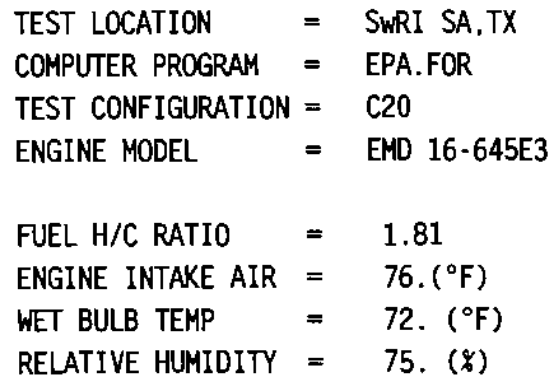

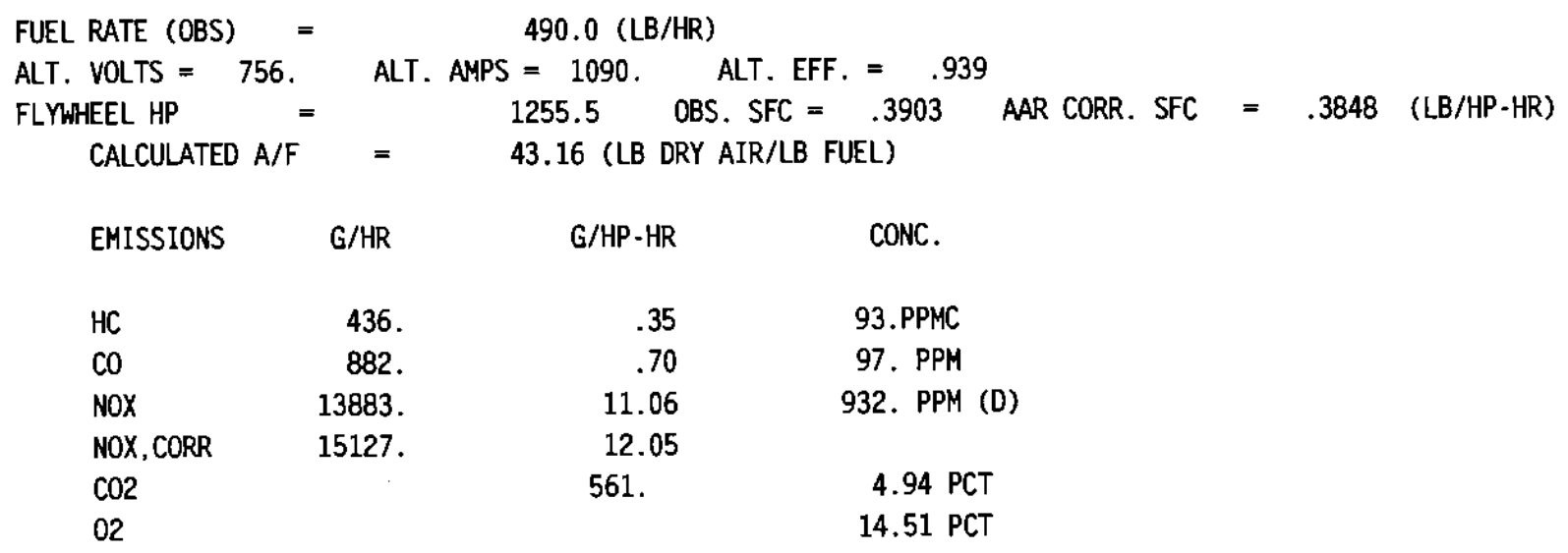

CORRECTION FACTOR SUMMARY:

$\begin{array}{lllll}\text { EPA NOX CF }=1.0897 & \text { NOX-KH } & =1.0732 & \text { NOX-KT } & =1.0000 \\ \text { WET EXH HC CF }=.9522 & \text { AIR TEMP CF }=.9928 & \text { BAROM CF } & =1.0035 \\ \text { FUEL TEMP CF }=.9860 & \text { FUEL S.G. CF }=1.0049 & \text { FUEL HHV CF } & =.9896\end{array}$

PARTICULATE INFORMATION

FILTER PAIR I.D. NUMBER

$6738.0-23$

FILTER PAIR WEIGHT GAIN (mg)

8.511

SAMPLE VOLUME (sCf)

37.543

DILUTE PM CONCENTRATION (mg/scf)

.227

CO2-BASED DILUTION FACTOR

6.398

PARTICULATE CONCENTRATION IN RAW EXHAUST (mg/scf)

1.450

PARTICULATE MASS EMISSION RATE $(\mathrm{g} / \mathrm{hr})$

418.

BRAKE-SPECIFIC PARTICULATE EMISSION RATE $(\mathrm{g} / \mathrm{hp} \cdot \mathrm{hr})$ 


$\begin{array}{ll}\text { LOCOMOTIVE MODEL } & =\text { EMD GP38 } \\ \text { LOCOMOTIVE UNIT \# } & =\text { CSX \#2629 } \\ \text { THROTILE NOTCH } & =\text { Notch } 6 \\ \text { TEST DATE } & =10 / 27 / 0 \\ \text { TEST NUMBER } & =8 / 10 \\ \text { SWRI FUEL CODE } & =\text { EM-3005-F } \\ \text { BAROMETER } & =29.26(\text { in. Hg) } \\ \text { DRY BULB TEMP } & =79 .\left({ }^{\circ} \mathrm{F}\right) \\ \text { ABS HUMIDITY } & =109.08(\text { GR/LB })\end{array}$

ENGINE PERFORMANCE SUMMARY:

ACCESSORY HORSEPOWER:

$\begin{array}{rlr}\text { AUXILLARY GENERATOR HP } & = & 4.0 \\ \text { AIR COMPRESSOR HP } & = & 12.2 \\ \text { TRACTION MOTOR BLOWER HP } & = & 44.1 \\ \text { INERTIAL SEPERATOR BLOWER HP } & =6.6 \\ \text { RADIATOR FAN HP } & =40.8 \\ \text { TOTAL ACCESSORY HP } & =107.6\end{array}$

\begin{tabular}{|c|c|}
\hline TEST LOCATION & $=$ SWRI SA,TX \\
\hline COMPUTER PROGRAM & EPA.FOR \\
\hline TEST CONFIGURATION & $\mathrm{C} 20$ \\
\hline ENGINE MODEL & EMD $16.645 E$ \\
\hline C RATIO & 1.81 \\
\hline INTAKE AIR & $77 .\left({ }^{\circ} \mathrm{F}\right)$ \\
\hline B TFMP & 72. $\left({ }^{\circ} \mathrm{F}\right)$ \\
\hline & \\
\hline
\end{tabular}

FUEL RATE (OBS) $=610.0$ (LB/HR)

ALT. VOLTS $=833 . \quad$ ALT. AMPS $=1192 . \quad$ ALT. EFF. $=.939$

FLYWHEEL HP $=1526.1$ OBS. SFC $=.3997 \quad$ AAR CORR. SFC $=.3939(\mathrm{LB} / \mathrm{HP}-\mathrm{HR})$

CALCULATED A/F $=39.10$ (LB DRY AIR/LB FUEL)

EMISSIONS G/HR G/HP-HR CONC.

$\begin{array}{lrrr}\text { HC } & 653 . & .43 & 123 . \text { PPMC } \\ \text { CO } & 1469 . & .96 & 144 . \text { PPM } \\ \text { NOX } & 17719 . & 11.61 & 1057 . \text { PPM (D) } \\ \text { NOX, CORR } & 19213 . & 12.59 & \\ \text { C02 } & & 574 . & 5.46 \text { PCT } \\ 02 & & & 13.40 \text { PCT }\end{array}$

CORRECTION FACTOR SUMMARY:

$\begin{array}{lllll}\text { EPA NOX CF }=1.0843 & \text { NOX-KH } & =1.0689 & \text { NOX -KT } & =1.0000 \\ \text { WET EXH HC CF }=.9480 & \text { AIR TEMP CF }=.9923 & \text { BAROM CF } & =1.0035 \\ \text { FUEL TEMP CF }=.9855 & \text { FUEL S.G. CF }=1.0049 & \text { FUEL HHV CF } & =.9896\end{array}$

PARTICULATE INFORMATION

-...-

FILTER PAIR I.D. NUMBER

$6739.0 \cdot 24$

FILTER PAIR WEIGHT GAIN (mg)

8.732

SAMPLE VOLUME (scf)

37.202

DILUTE PM CONCENTRATION (mg/scf)

.235

CO2-BASED DILUTION FACTOR

7.527

PARTICULATE CONCENTRATION IN RAW EXHAUST (mg/scf)

1.767

PARTICULATE MASS EMISSION RATE $(\mathrm{g} / \mathrm{hr})$

575 .

BRAKE-SPECIFIC PARTICULATE EMISSION RATE $(\mathrm{g} / \mathrm{hp}-\mathrm{hr})$ 


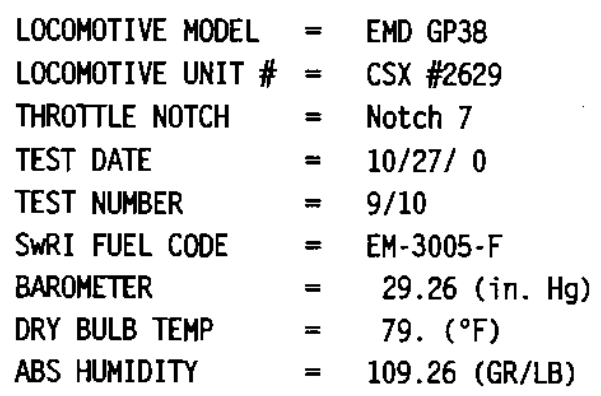

ENGINE PERFORMANCE SUMMARY:

ACCESSORY HORSEPOWER:

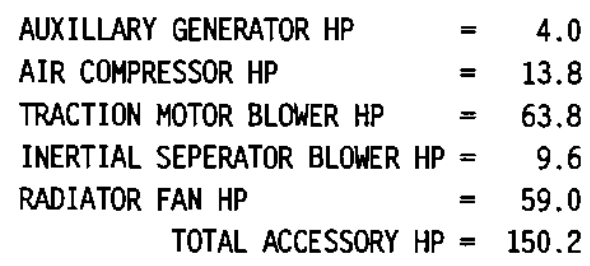

$02 \quad 12.52 \mathrm{PCT}$

CORRECTION FACTOR SUMMARY:

$\begin{array}{lllll}\text { EPA NOX CF }=1.0847 & \text { NOX-KH } & =1.0692 & \text { NOX-KT } & =1.0000 \\ \text { WET EXH HC CF }=.9423 & \text { AIR TEMP CF }=.9923 & \text { BAROM CF } & =1.0035 \\ \text { FUEL TEMP CF }=.9870 & \text { FUEL S.G. CF }=1.0049 & \text { FUEL HHV CF } & =.9896\end{array}$

PARTICULATE INFORMATION

FILTER PAIR I.D. NUMBER

FILTER PAIR WEIGHT GAIN (mg)

SAMPLE VOLUME (scf)

DILUTE PM CONCENTRATION ( $\mathrm{mg} / \mathrm{sCf}$ )

CO2-BASED DILUTION FACTOR

PARTICULATE CONCENTRATION IN RAW EXHAUST ( $\mathrm{mg} / \mathrm{sCf}$ )

PARTICULATE MASS EMISSION RATE $(\mathrm{g} / \mathrm{hr})$

BRAKE-SPECIFIC PARTICULATE EMISSION RATE $(g / h p-h r)$

$$
\begin{gathered}
6740.0 \cdot 25 \\
14.233 \\
36.514 \\
.390 \\
7.008 \\
2.732 \\
987 .
\end{gathered}
$$

.532 


$\begin{array}{ll}\text { LOCONOTIVE MODEL } & =\text { EMD GP38 } \\ \text { LOCOMOTIVE UNIT \# } & =\text { CSX \#2629 } \\ \text { THROTTLE NOTCH } & =\text { Notch } 8 \\ \text { TEST DATE } & =10 / 27 / 0 \\ \text { TEST NUMBER } & =10 / 10 \\ \text { SWRI FUEL CODE } & =\text { EM }-3005 \cdot \mathrm{F} \\ \text { BAROMETER } & =29.26(\mathrm{in} . \mathrm{Hg}) \\ \text { DRY BULB TEMP } & =79 .\left(^{\circ} \mathrm{F}\right) \\ \text { ABS HUMIDITY } & =108.84(\mathrm{GR} / \mathrm{LB})\end{array}$

ENGINE PERFORMANCE SUMMARY:

ACCESSORY HORSEPOWER:

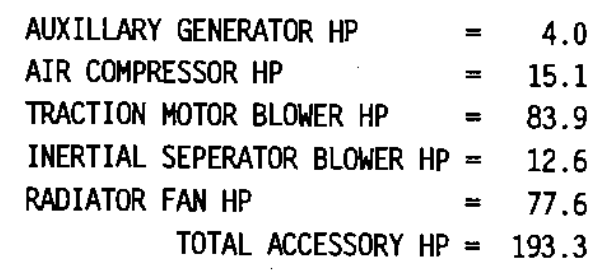

$\begin{array}{rrr}\text { AUXILLARY GENERATOR HP } & =4.0 \\ \text { AIR COMPRESSOR HP } & =15.1 \\ \text { TRACTION MOTOR BLOWER HP } & =83.9 \\ \text { INERTIAL SEPERATOR BLOWER HP } & =12.6 \\ \text { RADIATOR FAN HP } & =77.6 \\ \text { TOTAL ACCESSORY HP } & =193.3\end{array}$

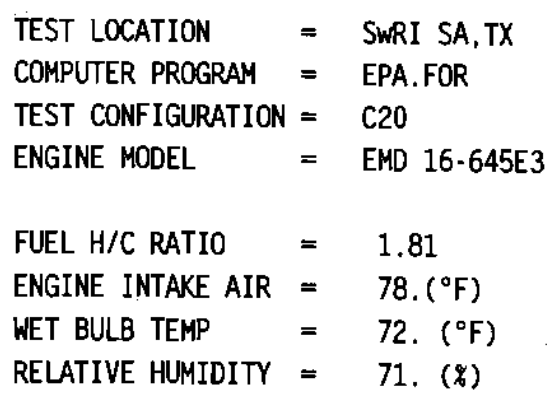




\begin{tabular}{|c|c|c|c|c|c|c|c|c|c|c|c|c|c|c|c|c|c|c|c|c|}
\hline$\infty$ & & v & $\begin{array}{l}0 \\
-1\end{array}$ & $\bar{N}$ & $\infty$ & 8 & $\infty$ & $\frac{s}{a}$ & $\frac{5}{\sigma}$ & $\underline{n}$ & $\begin{array}{l}2 \\
\infty \\
\infty\end{array}$ & N & $\begin{array}{l}n \\
n \\
n\end{array}$ & $\begin{array}{l}\mathbf{X} \\
\mathcal{N} \\
\mathcal{O} \\
\mathcal{O}\end{array}$ & $\begin{array}{l}\infty \\
3 \\
3\end{array}$ & $\begin{array}{l}\hat{n} \\
\alpha \\
\Sigma \\
\approx\end{array}$ & $\begin{array}{l}0 \\
2\end{array}$ & $\frac{0}{8}$ & in & 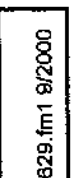 \\
\hline 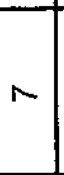 & & ry & $\theta$ & $\begin{array}{c}0 \\
N \\
n\end{array}$ & $\infty$ & $M$ & $N$ & $\underset{N}{\alpha}$ & $\frac{n}{\sigma}$ & $\begin{array}{l}\text { D } \\
\text { m }\end{array}$ & $N$ & nf & $\begin{array}{l}0 \\
D \\
\text { N } \\
\text { S }\end{array}$ & $\begin{array}{l}2 \\
x^{-} \\
\infty \\
7\end{array}$ & $\frac{m}{9}$ & $\begin{array}{l}\text { n. } \\
2 \\
2 \\
=\end{array}$ & $\begin{array}{l}\mathfrak{N} \\
\mathfrak{N} \\
\mathfrak{N}\end{array}$ & $\begin{array}{l}n \\
\infty \\
\infty\end{array}$ & $\not{x}$ & 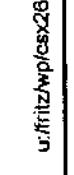 \\
\hline$\omega$ & & $\begin{array}{l}\text { vै } \\
\text { ज्ञ }\end{array}$ & $N$ & $\begin{array}{l}0 \\
\tilde{x} \\
r\end{array}$ & $\sigma$ & $N$ & & m & $m$ & $\Rightarrow$ & $\frac{0}{3}$ & $N$ & $\begin{array}{l}N \\
N \\
N\end{array}$ & $\begin{array}{l}\sigma \\
\tilde{v} \\
\tau\end{array}$ & $\underset{v}{*}$ & $\begin{array}{l}0 \\
0 \\
\dot{y} \\
5 \\
\end{array}$ & $\begin{array}{l}0 \\
n \\
n\end{array}$ & 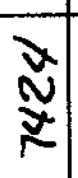 & $\infty$ & \\
\hline م & & $\begin{array}{l}y \\
y \\
y\end{array}$ & 0 & $\begin{array}{l}0 \\
N\end{array}$ & $\begin{array}{l}\infty_{0} \\
\infty_{0}\end{array}$ & $\infty$ & $N$ & $N$ & $\stackrel{3}{N}$ & 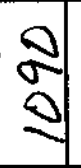 & $\begin{array}{l}0 \\
\sigma\end{array}$ & $n$ & $\begin{array}{l}\sqrt{g} \\
\hat{v}\end{array}$ & $\begin{array}{l}w \\
\tilde{\sigma} \\
\sigma\end{array}$ & $\frac{\pi}{2}$ & $\frac{\pi}{n}$ & $\begin{array}{l}\overrightarrow{2} \\
\vec{v}\end{array}$ & $\begin{array}{l}N \\
\mathbb{N} \\
\boldsymbol{N}\end{array}$ & $\underset{n}{\infty}$ & \\
\hline$\nabla$ & & गु & N & $\begin{array}{l}\infty \\
=\end{array}$ & $m$ & o & $\pi$ & $\frac{7}{6}$ & $\infty$ & $\begin{array}{l}7 \\
\infty \\
0\end{array}$ & $\begin{array}{l}J \\
m\end{array}$ & & $\begin{array}{l}5 \\
5\end{array}$ & $\begin{array}{c}5 \\
0 \\
9\end{array}$ & $m$ & $\begin{array}{l}m \\
\infty \\
\dot{0} \\
\infty\end{array}$ & $\begin{array}{l}0 \\
0 \\
2\end{array}$ & 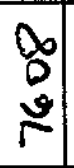 & 合 & \\
\hline$m$ & & 검 & v & $\ddot{n}$ & $m$ & $y$ & $m$ & $\begin{array}{l}0 \\
6 \\
6\end{array}$ & $\begin{array}{l}m \\
h\end{array}$ & $N$ & $\begin{array}{r}r \\
M\end{array}$ & & $\begin{array}{l}3 \\
\mathbf{s}\end{array}$ & $\begin{array}{l}0 \\
3 \\
3\end{array}$ & $\frac{n}{\sigma}$ & 㫄 & $\begin{array}{l}0 \\
0 \\
0\end{array}$ & $\begin{array}{l}0 \\
n \\
n\end{array}$ & $\begin{array}{l}7 \\
0 \\
9\end{array}$ & \\
\hline$N$ & & जे & $\begin{array}{l}N \\
N\end{array}$ & $\bar{n}$ & N & $N$ & $n$ & $\begin{array}{l}a \\
m\end{array}$ & $\rightarrow$ & a & m & & $\begin{array}{l}8 \\
3 \\
\text { กิ }\end{array}$ & $\begin{array}{l}\hat{b} \\
0 \\
+\end{array}$ & $\frac{\infty}{N}$ & $\begin{array}{l}9 \\
8 \\
8 \\
0\end{array}$ & $\tilde{\sigma}$ & $\frac{\infty}{\mathbf{W}}$ & ñ & \\
\hline- & & $\begin{array}{l}9 \\
8 \\
8\end{array}$ & 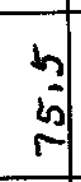 & $\vec{y}$ & $m$ & I & 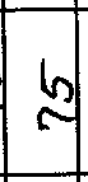 & $\frac{n}{m}$ & $\infty$ & $\begin{array}{l}\infty \\
-1\end{array}$ & $\begin{array}{l}\mathrm{n} \\
\text { nf }\end{array}$ & & 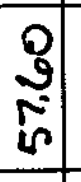 & $\frac{5}{8}$ & 8 & sos & $\begin{array}{l}n \\
\sigma \\
\sigma\end{array}$ & $\begin{array}{l}N \\
\text { in } \\
n\end{array}$ & $\begin{array}{l}\not \\
3\end{array}$ & \\
\hline $\begin{array}{l}\text { No } \\
\text { 它 }\end{array}$ & & $\begin{array}{l}n \\
\text { 궁 }\end{array}$ & ज & $\frac{M}{n}$ & 6 & 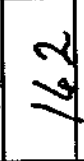 & $h$ & $\begin{array}{l}\infty \\
m\end{array}$ & $N$ & 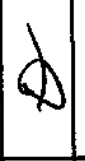 & $\begin{array}{l}0 \\
\text { m }\end{array}$ & & $\begin{array}{l}= \\
\vdots \\
3\end{array}$ & $\begin{array}{l}n \\
2 \\
3\end{array}$ & $\stackrel{N}{\sim}$ & $\begin{array}{l}n \\
\stackrel{4}{6} \\
\overline{0}\end{array}$ & $\begin{array}{c}n \\
0 \\
0 \\
0\end{array}$ & $\frac{\nabla}{\mathfrak{N}}$ & $\begin{array}{l}n \\
m \\
\text { in }\end{array}$ & \\
\hline$\frac{\Phi}{\underline{\underline{D}}}$ & & v & $\vec{\jmath}$ & $\vec{H}$ & 5 & $g$ & $\Gamma$ & $\begin{array}{l}\sigma \\
\sigma\end{array}$ & $\begin{array}{l}\alpha \\
\infty\end{array}$ & $\phi$ & $\begin{array}{l}10 \\
15\end{array}$ & $\infty$ & 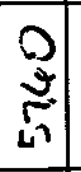 & $\stackrel{r}{r}$ & J & $\begin{array}{l}N \\
0 \\
0\end{array}$ & $\begin{array}{l}n \\
0 \\
0 \\
0\end{array}$ & $\frac{5}{N}$ & $\begin{array}{l}7 \\
8 \\
3\end{array}$ & \\
\hline $\begin{array}{l}\text { 등 } \\
\text { 은 }\end{array}$ & 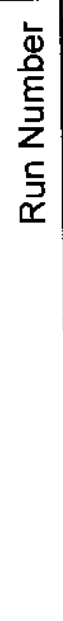 & 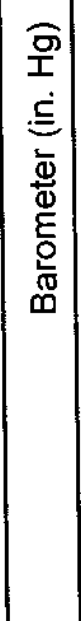 & 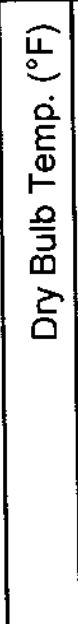 & 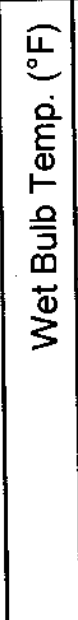 & 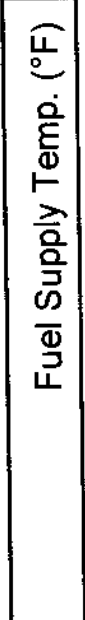 & 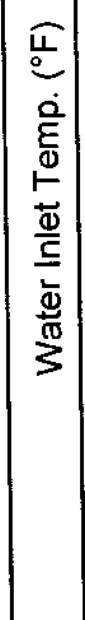 & 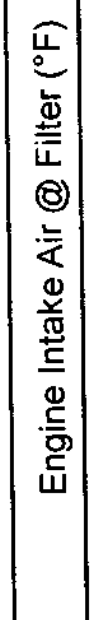 & $\begin{array}{c}\widehat{E} \\
\text { 은 } \\
\overline{0} \\
\Phi \\
\Phi \\
0 \\
0 \\
\Phi \\
\stackrel{5}{0} \\
\bar{w}\end{array}$ & 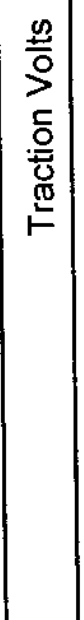 & 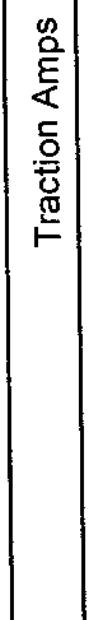 & 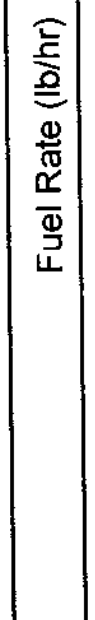 & 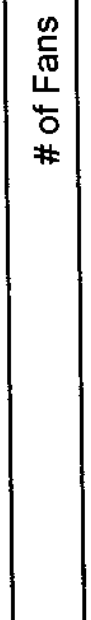 & $\begin{array}{l}\widehat{O} \\
\varepsilon \\
\text { 응 } \\
\text { U) } \\
\text { I }\end{array}$ & $\begin{array}{l}\widehat{\varepsilon} \\
\text { 응 } \\
\text { 엉 }\end{array}$ & 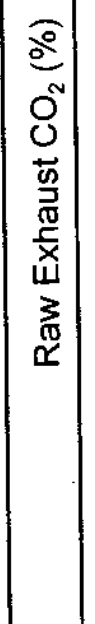 & $\begin{array}{l}\widehat{\xi} \\
\underline{0} \\
\text { ․ } \\
\mathrm{O}^{x}\end{array}$ & $\begin{array}{c}\frac{\varrho}{2} \\
0^{N}\end{array}$ & 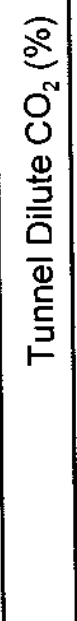 & 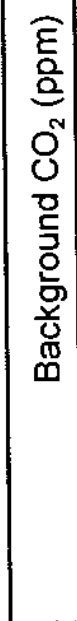 & 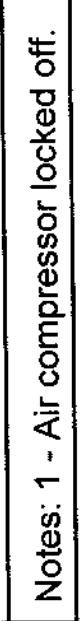 \\
\hline
\end{tabular}




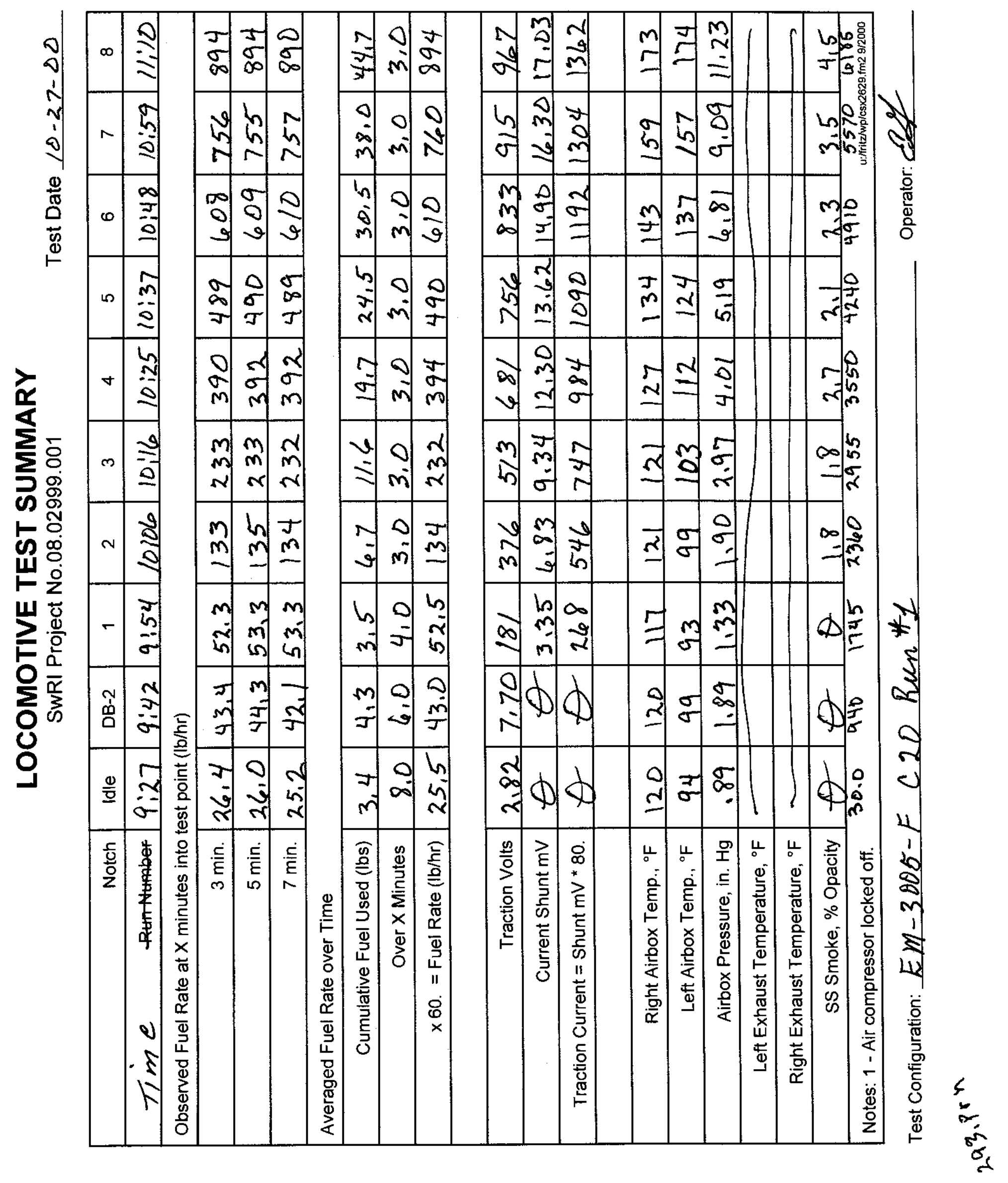




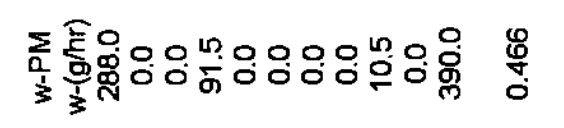

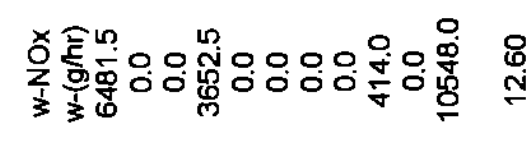

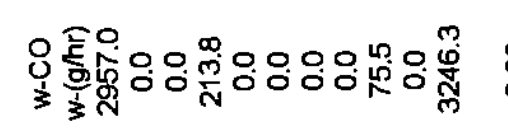

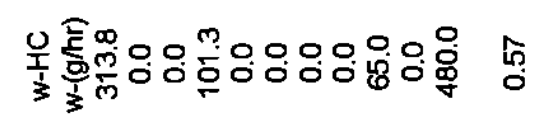

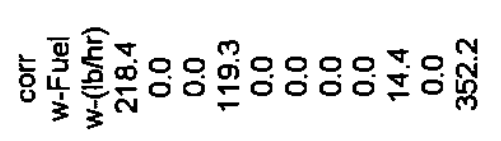

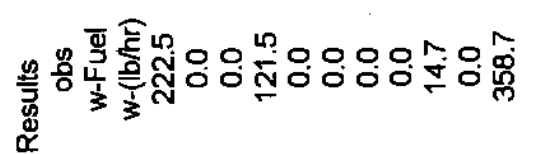

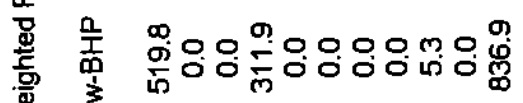
$\sum^{2}$ 柆

\section{ㄴํㅇํㅇำ}

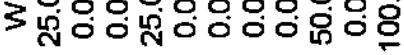

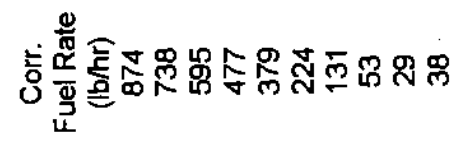

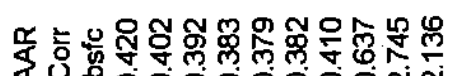

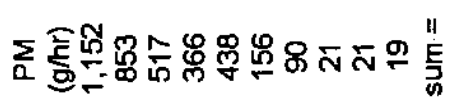

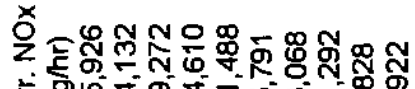

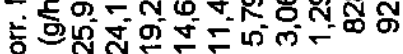

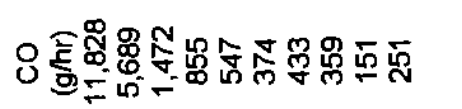

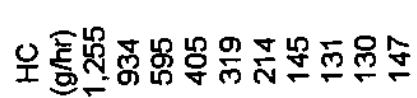

용 $\sum_{u}^{\infty}$

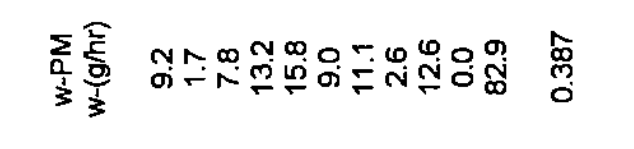

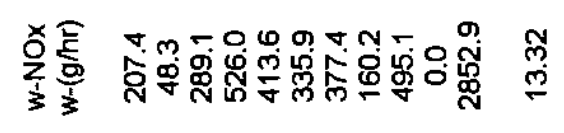

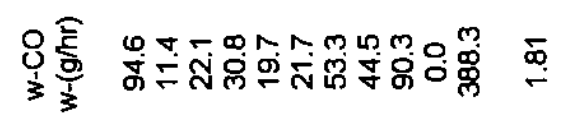

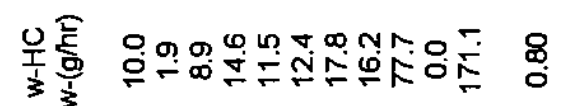

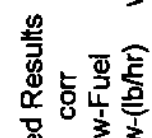

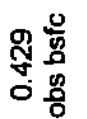

兽

oे

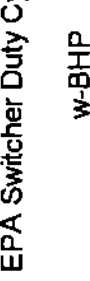

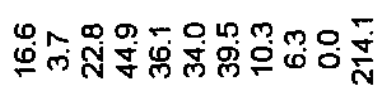

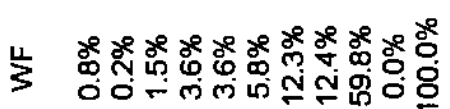

高紊

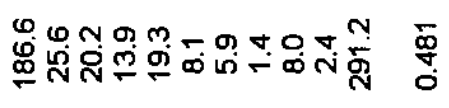

絃害

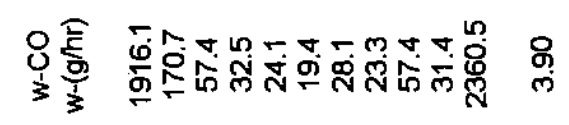

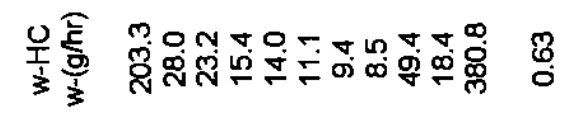

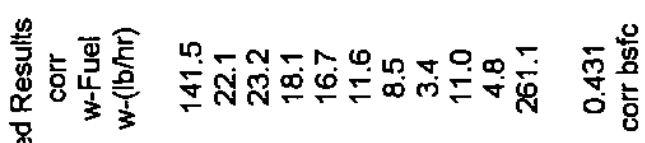$$
\text { 曾 }
$$

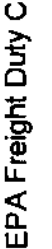

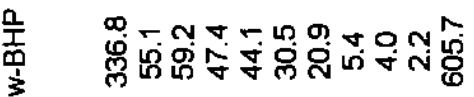

З 
LOCOMOTIVE EMISSIONS SWRI PROJECT NO. 08-2999-001

$\begin{array}{llll}\text { LOCOMOTIVE MOOEL } & =\text { EMD GP38 } & \text { TEST LOCATION } & =\text { SWRI SA,TX } \\ \text { LOCOMOTIVE UNIT \# } & \text { CSX \#2629 } & \text { COMPUTER PROGRAM } & =\text { EPA. FOR } \\ \text { THROTTLE NOTCH } & =\text { IDLE } & \text { TEST CONFIGURATION } & \text { C20 } \\ \text { TEST DATE } & =10 / 30 / 0 & \text { ENGINE MODEL } & = \\ \text { TEST NUMBER } & =1 / 10 & & \\ \text { SWRI FUEL CODE } & =\text { EM-3005-F } & \text { FUEL H/C RATIO } & =1.84 \\ \text { BAROMETER } & =29.19(\mathrm{in} . \mathrm{Hg}) & \text { ENGINE INTAKE AIR } & =90 .\left(^{\circ} \mathrm{F}\right) \\ \text { DRY BULB TEMP } & =86 .\left({ }^{\circ} \mathrm{F}\right) & \text { WET BULB TEMP } & =70 .\left(^{\circ} \mathrm{F}\right) \\ \text { ABS HUMIDITY } & =87.22(\mathrm{GR} / \mathrm{LB}) & \text { RELATIVE HUMIDITY } & =45 .\left({ }^{\circ}\right)\end{array}$

ENGINE PERFORMANCE SUMMARY:

ACCESSORY HORSEPOWER:

$\begin{array}{rrr}\text { AUXILLARY GENERATOR HP } & = & 4.0 \\ \text { AIR COMPRESSOR HP } & = & 4.3 \\ \text { TRACTION MOTOR BLOWER HP } & =1.9 \\ \text { INERTIAL SEPERATOR BLOWER HP } & =.3 \\ \text { RADIATOR FAN HP } & = \\ \text { TOTAL ACCESSORY HP } & =10.5\end{array}$

FUEL RATE (OBS)

ALT. VOLTS $=$ $=$

$29.3(\mathrm{LB} / \mathrm{HR})$

FLYWHEEL HP

3.

ALT. AMPS $=$

0. ALT. EFF. $=.939$

CALCULATED A/F

10.5

OBS. $\mathrm{SFC}=2.8034$

AAR CORR. SFC $=2.7447$ (LB/HP-HR)

EMISSIONS

G/HR

G/HP-HR

CONC.

$\begin{array}{llrc}\text { HC } & 130 . & 12.48 & 65 . \text { PPMC } \\ \text { CO } & 151 . & 14.44 & 38 . \text { PPM } \\ \text { NOX } & 802 . & 76.70 & 122 . \text { PPM (D) } \\ \text { NOX, CORR } & 828 . & 79.26 & \\ \text { C02 } & & 3982 . & .66 \text { PCT } \\ 02 & & & 20.03 \text { PCT }\end{array}$

CORRECTION FACTOR SUMMARY:

EPA NOX CF $=1.0334$

WET EXH HC CF $=.9899$

NOX-KH $\quad=1.0274$

NOX-KT $\quad=1.0000$

FUEL TEMP CF $=.9 \mathrm{~B} 00$

AIR TEMP CF $=.9865$

BAROM CF $=1.0029$

FUEL S.G. $C F=1.0049$

FUEL. HHV CF $=.9896$

PARTICULATE INFORMATION

FILTER PAIR I.D. NUMBER

$6782.0-67$

1.047

FILTER PAIR WEIGHT GAIN (mg)

25.842

SAMPLE VOLUME (sCf)

DILUTE PM CONCENTRATION (mg/scf)

.041

4.306

CO2-BASED DILUTION FACTOR

.174

PARTICULATE MASS EMISSION RATE $(\mathrm{g} / \mathrm{hr})$

21.

BRAKE-SPECIFIC PARTICULATE EMISSION RATE $(\mathrm{g} / \mathrm{hp} \cdot \mathrm{hr})$

2.046 


$\begin{array}{ll}\text { LOCOMOTIVE MODEL } & =\text { EMD GP38 } \\ \text { LOCOMOTIVE UNIT \# } & =\text { CSX \#2629 } \\ \text { THROTLE NOTCH } & =\text { DB2 } \\ \text { TEST DATE } & =10 / 30 / 0 \\ \text { TEST NUM8ER } & =2 / 10 \\ \text { SWRI FUEL CODE } & =\text { EM-3005-F } \\ \text { BAROMETER } & =29.19(\text { in. Hg) } \\ \text { DRY BULB TEMP } & =85 .\left({ }^{\circ} \mathrm{F}\right) \\ \text { ABS HUMIDITY } & =89.04(\text { GR } / L B)\end{array}$

ENGINE PERFORMANCE SUMMARY:

ACCESSORY HORSEPOWER:

$\begin{array}{rrr}\text { AUXILLARY GENERATOR HP } & = & 4.0 \\ \text { AIR COMPRESSOR HP } & = & 6.4 \\ \text { TRACTION MOTOR BLOWER HP } & = & 6.5 \\ \text { INERTIAL SEPERATOR BLOWER HP } & = & 1.0 \\ \text { RADIATOR FAN HP } & = & .0 \\ \text { TOTAL ACCESSORY HP } & =17.9\end{array}$

$\begin{array}{ll}\text { TEST LOCATION } & =\text { SWRI SA,TX } \\ \text { COMPUTER PROGRAM } & =\text { EPA.FOR } \\ \text { TEST CONFIGURATION } & =\text { C20 } \\ \text { ENGINE MODEL } & =\text { EMD } 16-645 E 3 \\ & \\ \text { FUEL H/C RATIO } & =1.81 \\ \text { ENGINE INTAKE AIR } & =90 .\left(^{\circ} \mathrm{F}\right) \\ \text { WET BULB TEMP } & =70 .\left({ }^{\circ} \mathrm{F}\right) \\ \text { RELATIVE HUMIDITY } & =48 .(\%)\end{array}$

CORRECTION FACTOR SUMMARY:

EPA NOX CF $=1.0384$

WET EXH HC CF $=.9893$

FUEL TEMP CF $=.9825$
NOX-KH $=1.0315$
AIR TEMP CF $=.9865$
FUEL S.G. $\mathrm{CF}=1.0049$

\section{PARTICULATE INFORMATION}

FILTER PAIR I.D. NUMBER

$6783.0-68$
1.194
39.013
.031
4.236
.130
19.
1.085




$\begin{array}{ll}\text { LOCOMOTIVE MODEL } & =\text { EMD GP38 } \\ \text { LOCOMOTIVE UNIT \# } & =\text { CSX \#2629 } \\ \text { THROTTLE NOTCH } & =\text { Notch } 1 \\ \text { TEST DATE } & =10 / 30 / 0 \\ \text { TEST NUMBER } & =3 / 10 \\ \text { SWRI FUEL CODE } & =\text { EM-3005-F } \\ \text { BAROMETER } & =29.19(\text { in. Hg) } \\ \text { DRY BULB TEMP } & =85 .\left({ }^{\circ} \mathrm{F}\right) \\ \text { ABS HUMIDITY } & =90.40(\text { GR/LB) }\end{array}$

ENGINE PERFORMANCE SUMMARY:

ACCESSORY HORSEPOWER:

$\begin{array}{rlr}\text { AUXILLARY GENERATOR HP } & = & 4.0 \\ \text { AIR COMPRESSOR HP } & = & 5.3 \\ \text { TRACTION MOTOR BLOWER HP } & =3.5 \\ \text { INERTIAL SEPERATOR BLOWER HP } & = \\ \text { RADIATOR FAN HP } & .5 \\ \text { TOTAL ACCESSORY HP } & =13.3\end{array}$

FUEL RATE (OBS) =

$54.0(\mathrm{LB} / \mathrm{HR})$

ALT, VOLTS $=182$.

ALT. AMPS $=269$.

ALT. EFF. $=.939$

FLYWHEEL HP =

83.2 OBS. $\mathrm{SFC}=.6491$

CALCULATED A/F $=192.36$ (LB DRY AIR/LB FUEL)

$\begin{array}{llll}\text { EMISSIONS } & G / H R & G / H P \cdot H R & \text { CONC. }\end{array}$

$\begin{array}{lrrr}\text { HC } & 131 . & 1.57 & 58 . \text { PPMC } \\ \text { C0 } & 359 . & 4.32 & 79 . \text { PPM } \\ \text { NOX } & 1241 . & 14.92 & 167 . \text { PPM (D) } \\ \text { NOX, CORR } & 1292 . & 15.53 & \\ \text { C02 } & & 925 . & 1.08 \text { PCT } \\ \text { 02 } & & & 19.53 \text { PCT }\end{array}$

CORRECTION FACTOR SUMMARY:

$\begin{array}{llll}\text { EPA NOX CF }=1.0409 & \text { NOX-KH }=1.0335 & \text { NOX-KT } & =1.0000 \\ \text { WET EXH HC CF }=.9860 & \text { AIR TEMP CF }=.9887 & \text { BAROM CF } & =1.0029 \\ \text { FUEL TEMP CF }=.9830 & \text { FUEL S.G. CF }=1.0049 & \text { FUEL HHV CF } & =.9896\end{array}$

PARTICULATE INFORMATION

FILTER PAIR I.D. NUMBER

$6784.0-69$

1.453

38.691

SAMPLE VOLUME (scf)

38.691

DILUTE PM CONCENTRATION (mg/sCf)

.038

3.980

C02-BASED DILUTION FACTOR

.149

21.

PARTICULATE MASS EMISSION RATE $(\mathrm{g} / \mathrm{hr})$

.250 


$\begin{array}{ll}\text { LOCOMOTIVE MODEL } & =\text { EMD GP38 } \\ \text { LOCOMOTIVE UNIT \# } & \text { CSX \#2629 } \\ \text { THROTLE NOTCH } & =\text { Notch } 2 \\ \text { TEST DATE } & =10 / 30 / 0 \\ \text { TEST NUMBER } & =4 / 10 \\ \text { SWRI FUEL CODE } & =\text { EM-3005-F } \\ \text { BAROMETER } & =29 . \mathrm{IB}(\mathrm{in} . \mathrm{Hg}) \\ \text { DRY BULB TEMP } & =85 .\left({ }^{\circ} \mathrm{F}\right) \\ \text { ABS HUMIDITY } & =88.97(\mathrm{GR} / \mathrm{LB})\end{array}$

ENGINE PERFORMANCE SUMMARY:

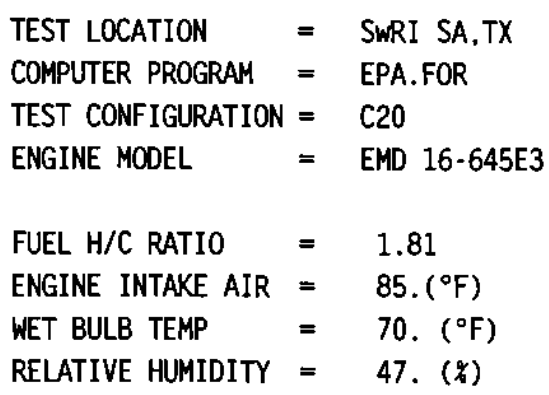

ACCESSORY HORSEPOWER:

$\begin{array}{rlr}\text { AUXILLARY GENERATOR HP } & =4.0 \\ \text { AIR COMPRESSOR HP } & =6.5 \\ \text { TRACTION MOTOR BLOWER HP } & =6.6 \\ \text { INERTIAL SEPERATOR BLOWER HP } & =1.0 \\ \text { RADIATOR FAN HP } & =3.1 \\ \text { TOTAL ACCESSORY HP } & =21.1\end{array}$

FUEL RATE (OBS)

134.0 (LB/HR)

ALT. VOLTS $=378$.

ALT. AMPS $=555$

ALT. EFF. $=.939$

FLYWHEEL HP

320.8

OBS. SFC $=.4177$

AAR CORR. SFC $=.4098(\mathrm{LB} / \mathrm{HP} \cdot \mathrm{HR})$

CALCULATED A/F $=93.86$ (LB DRY AIR/LB FUEL)

$\begin{array}{lccc}\text { EMISSIONS } & \text { G/HR } & \text { G/HP-HR } & \text { CONC. } \\ \text { HC } & 145 . & .45 & 53 . \text { PPMC } \\ \text { CO } & 433 . & 1.35 & 79 . \text { PPM } \\ \text { NOX } & 2966 . & 9.25 & 331 . \text { PPM (D) } \\ \text { NOX,CORR } & 3068 . & 9.56 & \\ \text { C02 } & & 599 . & 2.24 \text { PCT } \\ 02 & & & 17.91 \text { PCT }\end{array}$

CORRECTION FACTOR SUMMARY:

$\begin{array}{lllll}\text { EPA NOX CF }=1.0343 & \text { NOX-KH } & =1.0281 & \text { NOX-KT } & =1.0000 \\ \text { WET EXH HC CF }=.9759 & \text { AIR TEMP CF }=.9887 & \text { BAROM CF } & =1.0028 \\ \text { FUEL TEMP CF }=.9850 & \text { FUEL S.G. CF }=1.0049 & \text { FUEL HHV CF } & =.9896\end{array}$

PARTICULATE INFORMATION

FILTER PAIR I.D. NUMBER

$6785.0 \cdot 70$

FILTER PAIR WEIGHT GAIN (mg)

4.229

SAMPLE VOLUME (sCf)

38.405

DILUTE PM CONCENTRATION (mg/scf)

.110

CO2-BASED DILUTION FACTOR

4.854

PARTICULATE CONCENTRATION IN RAW EXHAUST (mg/scf)

PARTICULATE MASS EMISSION RATE $(\mathrm{g} / \mathrm{hr})$

.534

BRAKE-SPECIFIC PARTICULATE EMISSION RATE $(\mathrm{g} / \mathrm{hp}-\mathrm{hr})$

90. 
LOCOMOTIVE EMISSIONS SWRI PROJECT NO. 08-2999-001

$\begin{array}{llll}\text { LOCOMOTIVE MODEL } & =\text { EMD GP38 } & \text { TEST LOCATION } & =\text { SWRI SA, TX } \\ \text { LOCOMOTIVE UNIT \# } & =\text { CSX \#2629 } & \text { COMPUTER PROGRAM } & =\text { EPA.FOR } \\ \text { THROTTLE NOTCH } & =\text { Notch } 3 & \text { TEST CONFIGURATION } & \text { C20 } \\ \text { TEST DATE } & =10 / 30 / 0 & \text { ENGINE MODEL } & =\text { EMD } 16 \cdot 645 E 3 \\ \text { TEST NUMBER } & =5 / 10 & & \\ \text { SWRI FUEL CODE } & =\text { EM-3005-F } & \text { FUEL H/C RATIO } & =1.81 \\ \text { BAROMETER } & =29.18(\mathrm{in} . \mathrm{Hg}) & \text { ENGINE INTAKE AIR } & =84 .\left({ }^{\circ} \mathrm{F}\right) \\ \text { DRY BULB TEMP } & =84 .\left({ }^{\circ} \mathrm{F}\right) & \text { WET BULB TEMP } & =70 .\left({ }^{\circ} \mathrm{F}\right) \\ \text { ABS HUMIDITY } & =87.03(\mathrm{GR} / \mathrm{LB}) & \text { RELATIVE HUMIDITY } & =49 .\left(\mathrm{y}^{\circ}\right)\end{array}$

ENGINE PERFORMANCE SUMMARY:

ACCESSORY HORSEPOWER:

$\begin{array}{rrr}\text { AUXILLARY GENERATOR HP } & = & 4.0 \\ \text { AIR COMPRESSOR HP } & =8.3 \\ \text { TRACTION MOTOR BLOWER HP } & =13.9 \\ \text { INERTIAL SEPERATOR BLOWER HP } & =2.1 \\ \text { RADIATOR FAN HP } & =6.4 \\ \text { TOTAL ACCESSORY HP } & =34.8\end{array}$

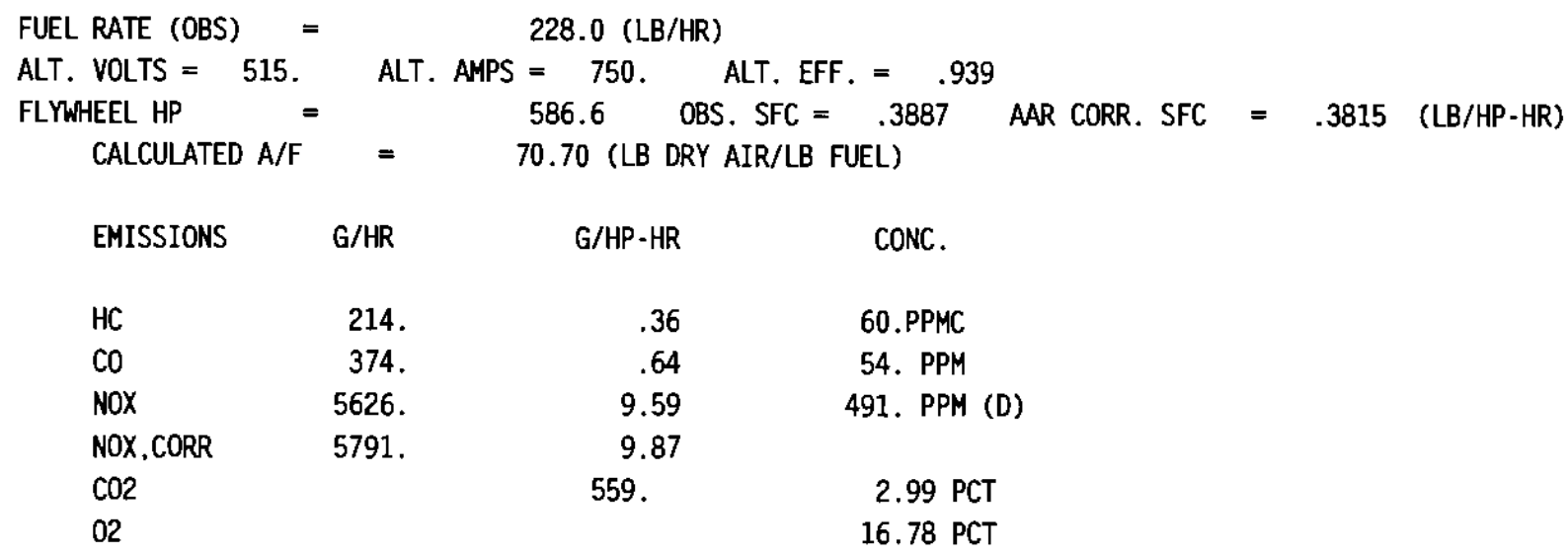

CORRECTION FACTOR SUMMARY:

$\begin{array}{lllll}\text { EPA NOX CF }=1.0292 & \text { NOX-KH } & =1.0240 & \text { NOX-KT } & =1.0000 \\ \text { WET EXH HC CF }=.9696 & \text { AIR TEMP CF }=.9892 & \text { BAROM CF } & =1.002 B \\ \text { FUEL TEMP CF }=.9865 & \text { FUEL S.G. CF }=1.0049 & \text { FUEL HHV CF } & =.9896\end{array}$

PARTICULATE INFORMATION

FILTER PAIR I.D. NUMBER

$6786.0-71$

5.570

38.236

.146

4.918

.716

156.

.266 


\section{APPENDIX E}

\section{SMOKE OPACITY TEST RESULTS}

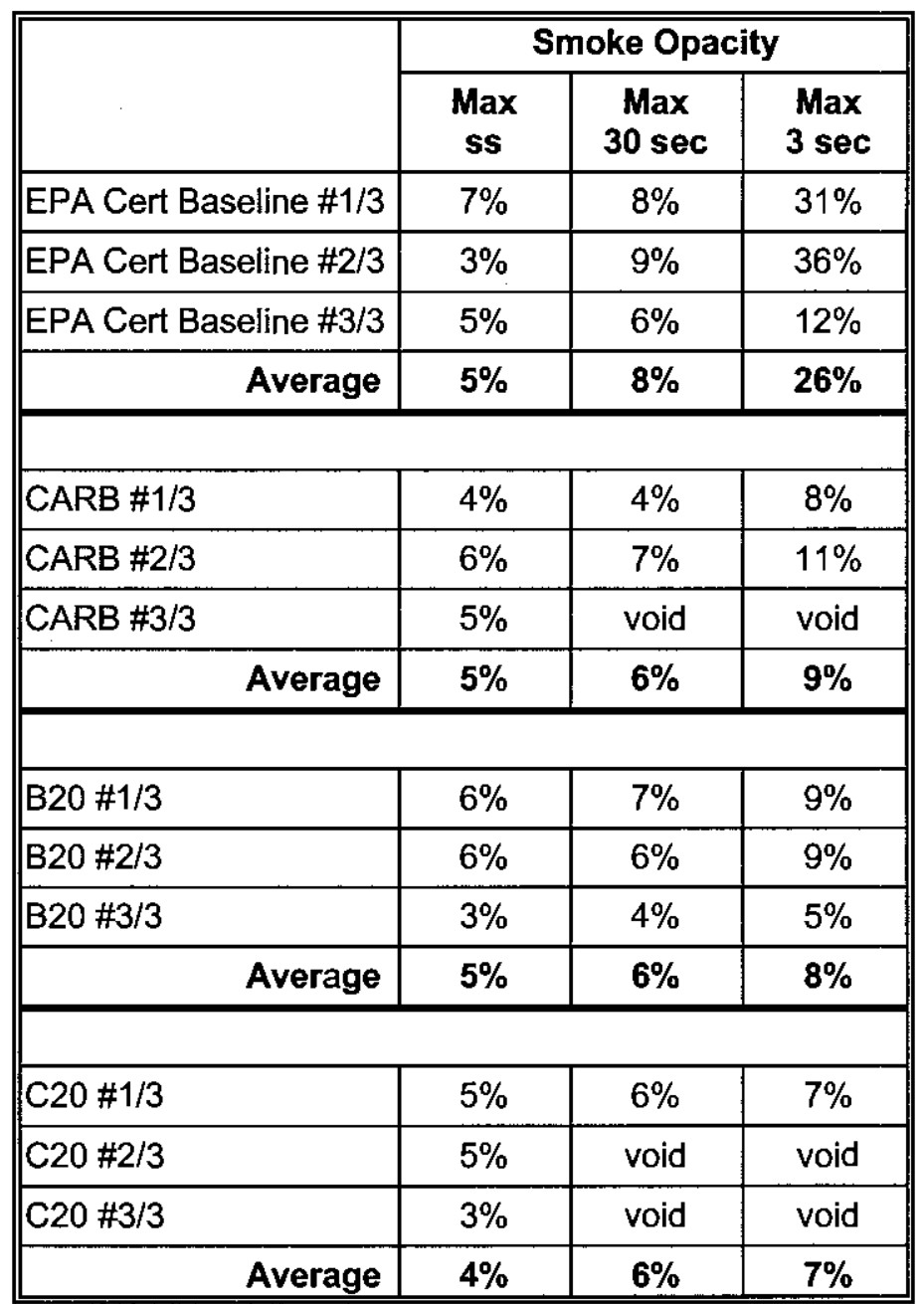




\section{APPENDIX F}

\section{LABORATORY ANALYSIS REPORT FOR NEAT BIO-FUEL}




\section{Laboratory Analysis Report}

Laboratory Services

Date Reponed: 6/4/01

Report To:

K. Shaine Tyson

Narional Renewable Rnergy Laboratory

1617 Cole Blvd

Subrnitred By:

Steve Fritz

Golden CO 80401

Southwest Research Instinue

6220 Culebra Rd

San Antonio TX 78238-5166

cC:

Type of Sanaple: Biodiesel

Sample ID: KS00110075 Sample Date: 10/31/2000

Sampla Description: $\quad$ Code \# EM-2997-F

Sampla Notes:

Mathod

Results

D93 Fasch Point $146^{\circ} \mathrm{C}$

D 3709 Water and Sediment

$<0: 02.5$ vol. $\%$

D 524

Carbon Residue, Ramsbottora

$0.06 \%$ Carbon Residue

D 874

Sulfated A.gh

0.001 mass \%

D 445

Kincmatic Viscosity

$4.798 \mathrm{cSt}$ @ $40^{\circ} \mathrm{C}$

D2622 Sulfur

0.0023 mass \% Sulfur

D613

Cetane Number

54.8 
D 130

D,664

Acid Number of Petroleum Products

$0.39 \mathrm{mg} \mathrm{KOH} / \mathrm{gram}$ by Potentiometric Titration

C. Plank
C. Plank

0.007 mass \% Free Glycerin

0.325 mass \% Monoglyceride

0.00 mass \% Diglyceride

0.00 mass \% Tsiglyceride

0.091 mass \% Glycerin 


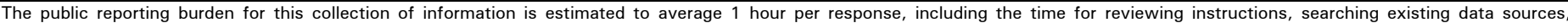

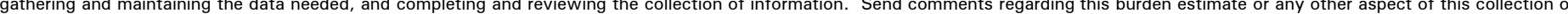

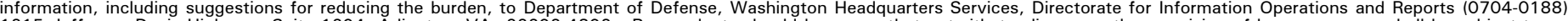

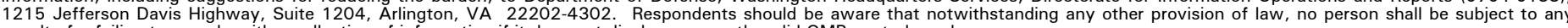
penalty for failing to comply with a collection of information if it does not display a currently valid OMB control number.

PLEASE DO NOT RETURN YOUR FORM TO THE ABOVE ADDRESS.
1. REPORT DATE (DD-MM- $Y Y Y Y)$
May 2004
Subcontract Report

4. TITLE AND SUBTITLE

Evaluation of Biodiesel Fuel in an EMD GP38-2 Locomotive
3. DATES COVERED (From - To)

5a. CONTRACT NUMBER

AXE-9-29057-01

5b. GRANT NUMBER

5c. PROGRAM ELEMENT NUMBER

5d. PROJECT NUMBER

5e. TASK NUMBER

BB044630

5f. WORK UNIT NUMBER

\section{PERFORMING ORGANIZATION NAME(S) AND ADDRESS(ES)}

Southwest Research Institute

San Antonio, TX

\section{SPONSORING/MONITORING AGENCY NAME(S) AND ADDRESS(ES)}

National Renewable Energy Laboratory

1617 Cole Blvd.

Golden, CO 80401-3393
8. PERFORMING ORGANIZATION REPORT NUMBER

10. SPONSOR/MONITOR'S ACRONYM(S)

NREL

11. SPONSOR/MONITOR'S REPORT NUMBER(S)

NREL/SR-510-33436

12. DISTRIBUTION/AVAILABILITY STATEMENT

\section{SUPPLEMENTARY NOTES}

NREL Technical Monitor: K.S. Tyson

\section{ABSTRACT}

This report documents results of fuel consumption and exhaust emission tests performed on a 2,000 hp EMD GP38-2 locomotive operating on four different fuels. No operational problems were observed by SwRI on any of the test fuels, and the locomotive engine was able to produce within $1 \%$ to $2 \%$ of full rated power while operating on the biodiesel blends.

15. SUBJECT TERMS

biodiesel; locomotive; SwRI

\begin{tabular}{|l|l|l|}
\hline 16. SECURITY CLASSIFICATION OF: \\
\hline a. REPORT & b. ABSTRACT & c. THIS PAGE \\
& & \\
\hline
\end{tabular}

\begin{tabular}{|l|l|l|}
\hline 17. LIMITATION OF & 18. NUMBER & $19 a$ \\
ABSTRACT & OF & \\
& PAGES & \\
\cline { 3 - 4 } & & $19 b$.
\end{tabular}

19a. NAME OF RESPONSIBLE PERSON

19b. TELEPHONE NUMBER (Include area code) 